\title{
EAP 2017 Congress and MasterCourse
}

\author{
October 12-15, 2017 \\ Ljubljana, Slovenia
}

\section{Oral Presentations}

\section{8}

Adolescent Medicine

Retrospective Analysis of Adolescent Outpatient Attendances Dhanis Lad ${ }^{1}$, Gavin Stone ${ }^{1}$, Uzair Khan ${ }^{1}$, Anne-Marie Murphy ${ }^{I}$

${ }^{T}$ Department of Paediatrics, University Hospital Limerick, Ireland

\section{Background}

Adolescence represents a unique time of transition to adulthood. Our hospital group encompasses 6 hospital sites catering to 100000 individuals under 18 years of age, approximately 18387 of which are aged between 14 and 16 years. Traditionally our Paediatric Department accepted new patients up until their 14th birthday, while those with chronic illnesses remained under Paediatric care until completion of second level education and were then transitioned to adult services. Our data addresses the concern of expanding the Paediatric field of inclusion to incorporate a growing number of adolescence and the impact this may have on the provisions of healthcare across many specialties.

\section{Objective}

The objective of our study is to compile data on outpatients between 14 and 16 years of age in our hospital group and to acknowledge the need to appropriately develop sustainable support models for this age group.

Methods

We looked at a 10 year study period between 2006 and 2016. Using the hospital's electronic database, we identified outpatient attendances of patients aged between 14 and 16 years. Details of patient demographics, symptomatology, clinic attendances and consultant(s) seen were documented.

Results

Over our 10-year study period 41456 patient contacts met the criteria for inclusion, 7828 (19\%) of these were under direct Paediatric care and 33 628 patients required assistance from other departments. Of the 25 departments offering assistance the most prevalent in order of frequency were Orthopaedics, Otolaryngology, Maxillofacial and Dermatology.

Conclusion

Our data highlights the demand placed on staff working in Paediatrics upon the extension of the Paediatric age profile. Future work to improve the quality of adolescent outpatient services should emphasize multidisciplinary team work whereby relevant specialties are working together under the leadership of an Adolescent Medicine Physician. Planning for health services is essential to ensure that clinical services are able to meet the current and future needs of the population it serves.

720

\section{Adolescent Medicine}

Adolescent Alcohol Intoxication and the Consumption of Energy Drink in the Netherlands - Is There a Problem? Karin Nienhuis ${ }^{I}$, Joris Van Hoof ${ }^{2}$, Nicolaas Van der Lely ${ }^{I}$

${ }^{T}$ Paediatrics, Reinier de Graaf Gasthuis, Netherlands

${ }^{2}$ Faculty of Behavioural Sciences, University of Twente, Netherlands

\section{Background}

In the past years, the consumption of energy drinks and alcohol mixed with energy drinks (AmED) has gained popularity in Europe and the United States. Consumption of AmED has various negative short term (i.e. injuries as a result of risk-taking behaviour) and long term (i.e. alcohol dependence later in life) effects. There is a maximum permitted quantity of caffeine in beverages in the Netherlands $(350 \mathrm{mg} / \mathrm{L})$, in contrast to countries like the United States and the United Kingdom (no maximum), where caffeine contents may be as high as $6100 \mathrm{mg} / \mathrm{L}$.

\section{Objective}

Direct clinical health effects of AmED consumption are hardly known. The aim of this study is to examine these effects.

Methods

For the past three years, data from all Dutch hospitals were collected, using the Dutch Paediatric Surveillance System (NSCK) database. Nationwide, every time an adolescent is admitted to one of the departments of paediatrics with an alcohol-related problem, a questionnaire is collected, which is implemented in the NSCK database.

\section{Results}

Two groups were created: adolescents who consumed energy drink at the event (ED+) and adolescents who did not (ED-). Blood alcohol concentration (BAC), hospital stay and duration of loss of consciousness did not differ significantly between both groups, nor did the mean age, sex ratio and habitual characteristics (age of first alcoholic beverage, smoking and alcohol consumption during weekend). In the ED+ group, there were significantly more adolescents in lower education and drugs were used twice as often.

\section{Conclusion}

Consumption of AmED with relatively low doses of caffeine does not lead to higher BAC in Dutch adolescents presented to the hospital with alcohol intoxication. As a side effect, adolescents in the ED+ group used drugs twice as often and were in lower education more often, a combination which is also seen in the general Dutch population.

\section{0}

\section{Adolescent Medicine}

An Audit of the Initial Health Assessment of Unaccompanied Asylum Seeking Children at a UK University Teaching Hospital Charles Paradise ${ }^{I}$, Varsha Sadavarte ${ }^{I}$

${ }^{T}$ Department of Community Paediatrics, University Hospital of North Midlands, UK

\section{BACKGROUND}

Unaccompanied children are extremely vulnerable, currently accounting for $8 \%$ of UK asylum applications.

\section{OBJECTIVE}

To examine if collective standards by RCPCH and a well-established expert UK region on the initial health assessment (IHA) of unaccompanied minors are being met.

\section{METHODS}

We studied 25 retrospective consecutive patients seen in 2016. The electronic BAAF (British Association Adoption and Fostering) form, and subsequent referral letters were reviewed. 


\section{RESULTS}

All were male teenagers. In majority country of origin was Afghanistan, Iraq or Iran. Presence of the social worker in only one IHA, and absence of interpreters in five of 22 cases shows lack of interagency communication. Although information documented covered general health questions in $80 \%$, with mental health screening reaching $96 \%$, we fell short in sexual health history. Risks including blood born virus (BBV), sexually transmitted disease exposure and route of travel was only documented in half. Positive findings by system included dermatological (44\%), emotional (40\%), dental (36\%), musculoskeletal and current smoker (28\% each) and sleep disturbance (16\%). Although BBV screening occurred in $88 \%$, HIV screening only occurred in half, and only $41 \%$ of 24 haemoglobinopathy screens were performed. Of the five vitamin D levels checked, $80 \%$ required treatment. Tuberculosis and mental health referral occurred in $96 \%$, however sexual education only occurred in $56 \%$. Of the 23 patients requiring immunisations, all GP's received detailed advice.

\section{CONCLUSION}

Despite clear guidelines, the use of BAAF forms allowed for vast individual interpretation in documentation. The review of these forms without correlation to the patient hospital notes is a limitation of this study. However, as these forms are used to instigate subsequent management plans, this is an important outcome. We developed a local guideline with flow charts and local service guide so staff completing IHAs were well equipped. We propose to re-audit this cohort in 12 months.

\section{2}

\section{Adolescent Medicine}

\section{Autonomy: The Ethical and Legal Issues facing Adolescents}

'You can bring a youth to college but you can't make him think' Kant Rachel Power'

${ }^{T}$ Postgraduate medicine, Royal College of Surgeons of Ireland, Ireland

\section{Background}

Autonomy is considered a major principle in making decisions about an individual's health. Children and particularly adolescents have the capacity to partake in medical decision-making to some extent. In most cases, the parent-doctor-adolescent triangle sides are in agreement, but this is not always the reality, causing ethical and legal issues for physicians to consider.

Objective

The overall aim is to evaluate the legal and ethical aspects of autonomy afforded to adolescents.

\section{Methods}

The databases perused for relevant papers were Pubmed and Westlaw. Based on their likelihood to produce hits, the Journal of Medical Ethics and Justis database were further examined.

\section{Results}

Autonomy is a basic human right, but not an absolute one. Contemporary challenges include arguments regarding the status of autonomy, often heralded by communitarian critiques versus individualist liberalists. Different legal decisions are defined by differing ages, which leads to confusion in terms of autonomy. Patients have a right to 'positive autonomy', i.e. to be facilitated in directing one's care, but they also have a right to 'negative autonomy'; i.e. the right to refuse treatment, and that is often more contentious, especially in adolescent medicine. In terms of the articulation of children's rights, the two most significant documents are the Constitution and the United Nations Convention on the Rights of the Child.

\section{Conclusion}

In the context of healthcare, the reality is that adolescents do not go through the same transitions at the same time. My own experience as a paediatric doctor has led me not to use chronological age as a determinant of capacity, as there is a continuum of development and we should hear the views of children and adolescents first before making any decisions.
503

\section{Adolescent Medicine}

The Impact of Chronic Pain in Adolescence Jennifer Roche ${ }^{1,2}$, Gavin Stone ${ }^{1}$, Anne-Marie Murphy ${ }^{1}$, Dominic Harmon $^{2}$

${ }^{1}$ Department of Paediatrics, University Hospital Limerick, Ireland

${ }^{2}$ Department of Pain Medicine and Anaesthetics, University Hospital Limerick, Ireland

\section{Background}

Chronic pain is prevalent among adolescents and negatively influences their quality of life. Furthermore, it may persist into adulthood. Multidisciplinary outpatient and intensive inpatient treatment has been shown to improve pain intensity and disability at this developmental stage.

\section{Objectives}

- To describe the inpatient and outpatient characteristics of an adolescent cohort with chronic pain,

- To explore their past and present pain management programmes,

- To highlight the impact of chronic pain at this developmental stage.

\section{Methods}

Data for this retrospective cohort (aged 12-19) study were obtained from the University Hospital Limerick Integrated Patient Management System (IPMS) and patient medical records were hand searched for the relevant information. Data was analysed using IBM SPSS Statistics version 22.

\section{Results}

A total of 21 patients ( $71 \%$ female) with chronic pain were identified from January 2016 through to February 2017. The mean age of these adolescent patients were 15.7 years. One of the most common presenting complaints and principal diagnoses was lower back pain. Comorbid diagnoses were common. Past and present pain management programmes included: assessment; therapeutic interview; education/self-management; exercise/physiotherapy/rehabilitation; pharmacology; psychology; pain intervention procedure and review. Chronic pain had a negative impact on patients general activities (notably school going), their enjoyment of life, mood; sleep and relationships.

\section{Conclusion}

Chronic pain is more prevalent in female adolescents and is complicated by a wide variety of comorbid conditions. These findings are consistent with recent literature. Different types of treatment example medical and psychological were used in the management of chronic pain in this adolescent cohort. This is in accordance with the biopsychosocial model which states that pain is caused by a complex interaction between biological, psychological and social variables.

603

\section{Adolescent Medicine}

Transitioning Youth from Paediatric to Adult Care: Knowledge, Practice and Perceptions of Paediatricians and Adult Physicians in Malta

Silvana Zammit ${ }^{l}$

${ }^{T}$ Paediatrics, Mater Dei Hospital, Malta

\section{Background}

Implementing an adolescent transition programme improves the clinical outcome of young persons with chronic conditions and reduces morbidity and mortality. There is no official adolescent transitional programme in Malta at present.

\section{Objectives}

To assess the knowledge, practice and perception of paediatricians and adult physicians regarding adolescent transitional care in Malta and to identify any barriers before a transitional programme can be implemented.

\section{Methods}

A mixed method approach was used. A modified questionnaire was developed from questionnaire tools in the literature. It was piloted and 
validated locally before being distributed to paeditricians and adult physicians at BST, HST, RS and consultant level in both departments. Descriptive analysis was applied. Semi-structured interviews were subsequently conducted and transcribed ad verbatim. Thematic analysis of the interview transcripts was conducted.

\section{Results}

The response rate for the questionnaire was $48.6 \%$ from adult physicians and $85.5 \%$ from paediatricians. Seventy-four percent of paediatricians and $82 \%$ of adult physicians have no training in ATC. Lack of training was the most significant barrier together with lack of clinic time, lack of resources and lack of a formalised transition policy. Interview analysis showed similarities with the findings of the questionnaire but conflicting views emerged regarding the role of the GP, whether provision of ATC is superior in the private sector or at $\mathrm{MDH}$ and the management of $\mathrm{YP}$ with a particular chronic condition in Malta. All the interviewees agreed on the importance of developing a standardised transition policy.

\section{Conclusions}

This is the first study exploring ATC in Malta. There are unmet education and training needs of paediatricians and adult physicians and a standardised adolescent transition care policy is needed..

A set of recommendations applicable to the Maltese system were drawn up from the results of this study that can help to set up an adolescent transitional care programme in Malta.

ATC - adolescent transitional care

GP - general practitioner

YP - young person

MDH - Mater Dei Hospital Malta

\section{0}

\section{Allergy \& Immunology}

Use of Adrenaline Autoinjectors in Paediatric Immunology Clinic A Single Centre Experience Joanna Start $^{1,2}$, Anil Shenoy ${ }^{1}$

${ }^{T}$ Department of Paediatric Immunology, Bradford Royal Infirmary, UK

${ }^{2}$ School of Medicine, University of Leeds, UK

\section{Background}

Anaphylaxis accounts for 1 in 1,000 admissions to paediatric units in the $\mathrm{UK}$ and this figure is increasing year on year. Immediate management of anaphylaxis includes antihistamines followed by intramuscular injection of adrenaline should symptoms continue. Adrenaline autoinjectors (AAIs) are prescribed to quicken treatment of anaphylaxis $(0.15 \mathrm{mg}$ for a young child or $0.3 \mathrm{mg}$ for a child or teenager). Literature around the number of AAIs prescribed in a paediatric setting is limited.

Objective

We aimed to quantify provision of AAIs in a paediatric centre and define the diagnoses, indications of prescribing and associated atopic comorbidities.

Methods

Retrospective analysis of the notes of 106 consecutive patients attending paediatric allergy clinic at a single centre between December 2015 and May 2016.

\section{Results}

Patient age range was $0-17$ years old; 72 patients were male and 34 female. 37 (35\%) patients had a current AAI, the final diagnosis of which included: food allergy -30 patients; idiopathic -2 patients; atopic disease -2 patients; venom allergy -1 patient; other allergy -2 patients. In the AAI group associated atopy included: 16 patients with eczema; 20 with asthma; and 1 with allergic rhinitis. Twenty-seven patients (73\%) provided with an AAI had a confirmed nut allergy. Thirty-four of the 37 AAI patients $(92 \%)$ were also prescribed an antihistamine. Within the whole cohort, there were 5 Emergency Department attendances due to allergy in the last year.

Conclusion

One in 3 patients had a current AAI. The most common indication was food allergy, specifically nut allergy. This may be explained by the higher prevalence of asthma in this setting. Prevalence of asthma and allergic rhinitis was highest in patients with a current AAI. It is important to review the prescription of AAIs regularly, with parental education on method of use. Management plans are provided to parents, nursery and schools.

24

\section{Critical/Emergency Care}

Inter-Regional Comparison of Accessibility of Children to the Nearest Hospital With At Least 5 Full-Time Pediatricians in Japan Akira Eharal

${ }^{T}$ Faculty of Health Services Management, Hiroshima International University, Japan

\section{Background}

2,656 hospital had pediatric department in Japan and 10,108 pediatricians worked in these facilities in 2014. Only 3.80 pediatricians worked in a hospital on average. Sick children in Japan do not visit a general practitioner but a pediatrician, and children must visit a big pediatric department in order to receive medical treatment on a 24-hour basis.

Objects

To know the number and percentage of children who lived within $30 \mathrm{~km}$, eqivalent to reachable areas within 1 hour by automobile, of the nearest hospital with at least 5 full-time pediatricians.

\section{Methods}

Japan is divided into 109,514 residential blocks under the Act on Indication of Residential Address. Child population under 15 years in each block was drawn from the 2010 Population Census. The list of hospitals with at least 5 full-time pediatricians as of 2014 was from the regional health authorities.

The number and percentage of children who lived in the residential blocks within $5,10,20,30$, and $50 \mathrm{~km}$ of the nearest pediatric department with at least 5 pediatricians were calculated with Geographic Information Systems.

Results

$90.2 \%$ of children in Japan lived within $30 \mathrm{~km}$ of the nearest pediatric department (Figure, Table). However, in underpopulated northern regions such as Hokkaido $(75.5 \%)$ and Tohoku $(59.1 \%)$, the percentile was lower compared with other regions.

Figure. Areas within $30 \mathrm{~km}$ of hospitals with at least 5 full-time pediatricians

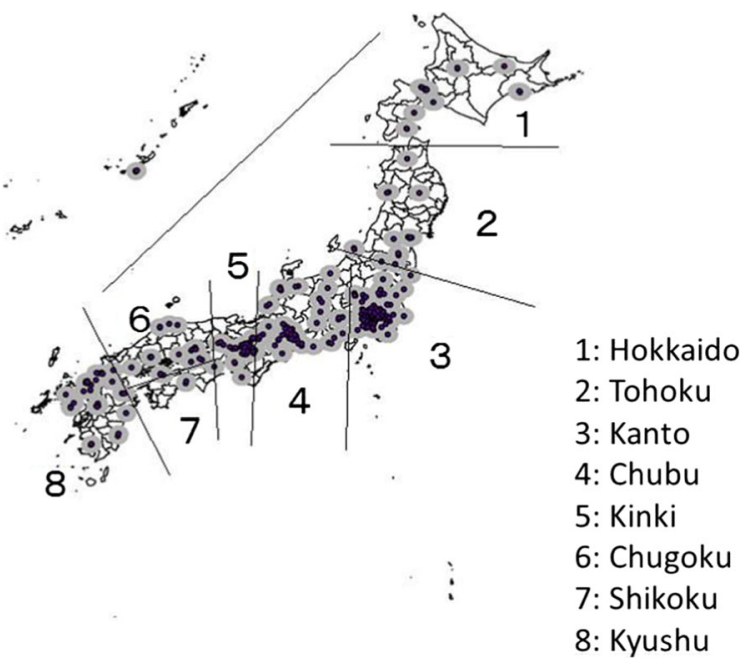


Table. Child population within the stated distance from the nearest hospital with 5 full-time pediatricians

\begin{tabular}{|c|c|c|c|c|c|c|c|}
\hline Region & & $<5 \mathrm{~km}$ & $<10 \mathrm{~km}$ & $<20 \mathrm{~km}$ & $<30 \mathrm{~km}$ & $<50 \mathrm{~km}$ & Total \\
\hline 1 Hokkaido & $\begin{array}{l}\text { Child Population } \\
\text { Rate }\end{array}$ & $\begin{array}{r}325.075 \\
49.5 \%\end{array}$ & $\begin{array}{r}422.229 \\
64.2 \%\end{array}$ & $\begin{array}{r}466.320 \\
70.9 \%\end{array}$ & $\begin{array}{l}496.376 \\
75.5 \%\end{array}$ & $\begin{array}{r}563.374 \\
857 \%\end{array}$ & $\begin{array}{r}657.312 \\
100.0 \%\end{array}$ \\
\hline & Child Population & 290.811 & 451.736 & 597.161 & 708,955 & 929,859 & 1.198 .736 \\
\hline 2 Tohoku & Rate & $24.3 \%$ & $37.7 \%$ & $49.8 n$ & $59.1 \%$ & $77.6 \%$ & $100.0 \%$ \\
\hline 3 Kanto & $\begin{array}{l}\text { Child Population } \\
\text { Rate }\end{array}$ & $\begin{array}{r}3,708.411 \\
69.1 \%\end{array}$ & $\begin{array}{r}4,649,607 \\
86.7 \%\end{array}$ & $\begin{array}{c}5,230,185 \\
97.5 \%\end{array}$ & $\begin{array}{r}5,344,998 \\
99,7 \%\end{array}$ & $\begin{array}{l}5,359,808 \\
99.9 \%\end{array}$ & $\begin{array}{r}5,363,114 \\
100.0 \%\end{array}$ \\
\hline 4 Chubu & $\begin{array}{l}\text { Child Population } \\
\text { Rate }\end{array}$ & $\begin{array}{l}1,253.175 \\
38.6 \%\end{array}$ & $\begin{array}{r}2,050,011 \\
63.2 \%\end{array}$ & $\begin{array}{r}2,629,792 \\
810^{\circ}\end{array}$ & $\begin{array}{c}2,965.282 \\
91.4 \%\end{array}$ & $\begin{array}{r}3,150,843 \\
97.1 \%\end{array}$ & $\begin{array}{r}3.245,949 \\
100.0 \%\end{array}$ \\
\hline 5 Kinki & $\begin{array}{l}\text { Child Population } \\
\text { Rate }\end{array}$ & $\begin{array}{r}1,772.957 \\
63.7 \%\end{array}$ & $\begin{array}{r}2.316 .632 \\
83.3 \%\end{array}$ & $\begin{array}{r}2.604 .984 \\
93.6^{\circ}\end{array}$ & $\begin{array}{r}2,674.201 \\
96.1 \%\end{array}$ & $\begin{array}{r}2.711 .031 \\
97.5 \%\end{array}$ & $\begin{array}{r}2.781 .690 \\
100.0 \%\end{array}$ \\
\hline 6 Chugoku & $\begin{array}{l}\text { Child Population } \\
\text { Rate }\end{array}$ & $\begin{array}{r}291.443 \\
29.0 \%\end{array}$ & $\begin{array}{r}501.625 \\
49.9 \%\end{array}$ & $\begin{array}{r}704.813 \\
70.1 \%\end{array}$ & $\begin{array}{r}823.288 \\
81.8 \%\end{array}$ & $\begin{array}{r}934.288 \\
92.9 \%\end{array}$ & $\begin{array}{r}1.005 .881 \\
100.0 \%\end{array}$ \\
\hline 7 Shikoku & $\begin{array}{l}\text { Child Population } \\
\text { Rate }\end{array}$ & $\begin{array}{r}153.685 \\
30.4 \%\end{array}$ & $\begin{array}{r}283.471 \\
56.0 \%\end{array}$ & $\begin{array}{r}353.747 \\
69.9 \%\end{array}$ & $\begin{array}{r}404.791 \\
80.0 \%\end{array}$ & $\begin{array}{r}467.661 \\
92.4 \%\end{array}$ & $\begin{array}{r}506.243 \\
100.0 \%\end{array}$ \\
\hline 8 Kyushu & $\begin{array}{l}\text { Child Population } \\
\text { Rate }\end{array}$ & $\begin{array}{r}875.781 \\
42.8 \% \\
\end{array}$ & $\begin{array}{r}1.215 .470 \\
59.5^{5} \% \\
\end{array}$ & $\begin{array}{r}1.565 .401 \\
76.6^{\circ} \\
\end{array}$ & $\begin{array}{r}1.731 .088 \\
84.7 \% \\
\end{array}$ & $\begin{array}{r}1.937 .293 \\
94.8 \% \\
\end{array}$ & $\begin{array}{r}2.044 .519 \\
100.0 \% \\
\end{array}$ \\
\hline Total & $\begin{array}{l}\text { Child Population } \\
\text { Rate }\end{array}$ & $\begin{array}{r}8.671 .338 \\
51.6 \%\end{array}$ & $\begin{array}{r}11.890,781 \\
70.8 \%\end{array}$ & $\begin{array}{r}14.152 .403 \\
84.2 \%\end{array}$ & $\begin{array}{c}15,148,979 \\
90.2 \%\end{array}$ & $\begin{array}{r}16.054 .157 \\
95.5 \%\end{array}$ & $\begin{array}{r}16.803 .444 \\
100.0 \%\end{array}$ \\
\hline
\end{tabular}

\section{Conclusion}

Most of children in Japan lived in the residential blocks within $30 \mathrm{~km}$ of hospitals with at least 5 full-time pediatricians. However, in underpopulated northern regions, accessibility was lower compared with other regions.

\section{8}

\section{Critical/Emergency Care}

Treatment option of partial thickness burn injuries in children Gergo Jozsa $^{1}$, Eszter Toth ${ }^{1}$, Zsolt Juhasz, ${ }^{1}$

${ }^{T}$ Unit of Pediatric Surgery, Department of Pediatrics, Medical Centre, University of Pécs, Hungary, Hungary

\section{Introduction}

There are several wound dressings available for the conservative treatment of second-degree burn injuries.

\section{Aims}

To determine the effectiveness of silver-laden foam and $\mathrm{Zn}$ hyaluronan gel for the treatment of partial thickness burns was carried out.

\section{Patients, methods}

Prospective study has been carried out between January 1, 2013 and December 1, 2015. A total of 73 children were treated with silver-laden foam and Zn-hyaluronan gel. For the first 24 hours, in order to assess the exact depth of the burn, $0.5 \%$ silver nitrate solution dressing was applied in $25 \%$ of the cases. In the rest of the children silver-laden foam with Zn-hyaluronan gel already as the first intervention was used. The dressing was removed on the seventh day.

\section{Results}

In the study population hot water scalds were the main cause of burn injury. Other causes included hot oil, flame, contact, and other types of burns. Wound size was $5 \%$ of the total body surface area on average. Burns were seen on all body parts; in 38 cases burns were noted in more than one area. Out of the 73 children were treated with this dressing. Wound infection was not noted. Epithelialization of the burned areas was observed on the 7 days after primary treatment.

\section{Conclusions}

The investigated dressing efficiently promotes epithelialization the burned areas. Further advantage of the Zn-hyaluronan gel is to enhance the regeneration of the dermal cells and inhibits the fixation of the dressing into the wound. Based on our experiences, we could achieve gentle, child-friendly, and cost-effective treatment, excellent wound healing and favorable cosmetic results in children.
361

\section{Critical/Emergency Care}

Sensitivity and specificity of caregivers' assessment of fever without a thermometer in a Paediatric Emergency Department

Carmel Maria Moore ${ }^{l}$, Niamh Walsh ${ }^{l}$, Roisin McNamara ${ }^{l}$, Nandini Kandamany ${ }^{I}$ Ikechukwu Okafor ${ }^{1}$

${ }^{I}$ Emergency Department, Temple Street Children's University Hospital, Ireland

NICE guidelines recommend that reported parental perception of a fever should be considered valid and taken seriously by healthcare professionals. This study aimed to assess the sensitivity and specificity of parental perception of pyrexia - without measuring with a thermometer upon attendance to the paediatric ED, as many parents were anecdotally noted to treat pyrexia without objective measurement.

\section{Methods}

This prospective observational study was approved by the institutional Ethics Board. Children of triage category 2 or lower who presented with a non-trauma related presentation were invited to participate through their parents. As part of nursing triage prior to measuring the child's temperature parents were asked if and why they thought that their child had a temperature in real time.

\section{Results}

$60 \%(n=168)$ of the 278 valid records returned were male, and $75 \%$ $(n=211)$ were Irish. More than half $(53 \%)$ of children had received antipyretics at home before presentation to the ED. Just over $7 \%$ $(n=20)$ were admitted as inpatients to the hospital. Only $12.9 \%$ of children included in the study were pyrexic (body temperature $\geq 38^{\circ}$ Celsius). Parents perceived pyrexia in $23.7 \%(n=66)$ children. Just under $84.5 \%$ of parents were correct in their assessment of their child's fever, giving parental assessment of pyrexia (without a thermometer) a sensitivity of $81 \%$ and a specificity of $85 \%$.

\section{Conclusion}

Caregivers' assessment of fever in paediatric patients attending our Emergency Department was sensitive and specific. Further analysis should be performed.

\section{5}

\section{Critical/Emergency Care}

Chest pain in pediatric emergency care: a retrospective analysis of causes and using of electrocardiogram

Carmela Santelia $^{I}$, Chiara Pilotto ${ }^{2}$, Anna Gozzi $i^{1}$, Michela Vergine ${ }^{I}$, Eva Passone ${ }^{1}$, Paola Cogo $^{1}$

${ }^{1}$ Departement of Maternal and Child Health ASUIUD S. Maria della Misericordia, Pediatric Clinic, Italy

${ }^{2}$ Department of Medical and Biological Science, University of Udine, Pediatric Clinic, Italy

\section{Background}

Chest pain is a relatively common and worrisome symptom in children often causing admission to emergency care. Estimated frequency ranges between $0.3-0.6 \%$. In the majority of cases the etiology is benign, differently to adults.

Objective

The aim of the study is to identife the most frequent causes of this symptom and the role of electrocardiogram (ECG) in the diagnostic process.

Methods

Retrospective case series of patients admitted to our pediatric emergency service was carried out to determine patients characteristic, risk factors, and diagnostic and therapeutical approach. Elegible patients were children from 3 months to 16 years of age admitted between 01/2006 and 12/ 2015. Descriptive analysis was performed to characterize the study population. Chi-square, Mann-Whitney U test or Fisher's exact test were used for comparison between groups as appropriate. Logistic regression 
was used to estimate odds ratios and $95 \%$ confidence intervals for all potential predictors.

\section{Results}

1078 patients ( $0.67 \%$ of total admission) were enrolled: 604 $(56.03 \%)$ were male, mean age (SD) at presentation was 9.8 years \pm 3.7 . Six different diagnostic cathegories were idientified: musculoskeletal in 707 patients $(68.57 \%)$, abdominal $(13.39 \%)$, psychosomatic $(8.34 \%)$ and respiratory $(6.50 \%)$; cardiac origin was founded only in 31 patients $(3.01 \%)$ and other causes in 2 patients $(0.02)$. Cardiac or respiratory physical exam alterations were predictive of a cardiac cause (RR 16.72 p0.0001), or a respiratory cause (RR 25.58 p0.0001). The ECG was performed in 794 patients $(73.65 \%)$ and showed alterations in 31 cases; all of them were admitted for a cardiac symptom (palpitation) or sign (p 0.0001).

\section{Conclusion}

Thoracic pain is a common complaint and rarely related with cardiac disorders. A physical examination is sufficient, in the vast majority of cases, to exclude a serious origin for the pain. Target diagnostic exams should be performed in selected patients.

\section{5}

\section{Critical/Emergency Care}

Infections and injuries of asylum seeking children in a refugee camp in Brussels in September 2015

Gerlant van Berlaer $^{1,2}$, Francisca Bohle Carbonell ${ }^{3}$, Sofie Manantsoa ${ }^{3}$, ${ }_{\text {Xavier de Béthune }}^{3}$, Ronald Buyl ${ }^{4}$, Michel Debacker ${ }^{1,2}$, Ives Hubloue ${ }^{1,2}$

${ }^{I}$ Department of Emergency Medicine, Universitair Ziekenhuis Brussel, Belgium

${ }^{2}$ Research Group on Emergency and Disaster Medicine, Vrije Universiteit Brussel, Belgium

${ }^{3}$ Medical Department, Operations Department,, Médecins du Mondel Doctors of the World Belgium, Belgium

${ }^{4}$ Department of Public Health, Biostatistics and Medical Informatics Research Group, Vrije Universiteit Brussel, Belgium

\section{Background}

In the summer of 2015, the exodus of Syrian war refugees and saturation of refugee camps in neighbouring countries led to the influx of many asylum-seekers in some European countries, including Belgium. This study aims to describe the demographic and clinical characteristics of the children among them.

Hypothesis is that among asylum-seeking children in a huddled refugee camp - even in a well-developed country with all medical facilities respiratory, digestive and skin infections typical of any refugee camp, will emerge.

Methods

Using a descriptive cross-sectional study design, physicians of Médecins du Monde (MdM) prospectively registered age, gender, origin, complaints and diagnoses of all paediatric patients presenting to an erected Field Hospital in Brussels during September 2015. Diagnoses were post-hoc categorized according to the International Classification of Diseases (ICD10).

\section{Results}

Of 3907 patients who presented to the Field Hospital, 395 (10\%) were children. Over $13 \%$ of patients suffered from injuries, but these were outnumbered by the proportion of patients with respiratory (39\%), digestive $(16 \%)$, skin $(14 \%)$ diagnoses. More than $57 \%$ had features of acute infections at the time, and one in ten had to be referred for more advanced care. Logistic regression indicates that children over five years old suffered significantly more from injuries, and children originating from Iraq and Syria had more infectious diseases compared with all other countries. We neither detected other significant differences between the age groups, nor any pathology pattern changes over time.

\section{Conclusions}

Asylum seeking children arriving in a refugee camp in Brussels after a long and hazardous journey, suffer mostly from respiratory, digestive, and skin diseases. Still one of seven suffers from injury. These findings - consistent with other reports on children in displaced persons camps worldwide - should be anticipated when health care workers plan the medical management and follow-up for these children, even in a Western European country.

\section{9}

\section{Critical/Emergency Care}

The evaluation of parental stress in the neonatal intensive care unit Gulcin Yapici $^{1}$, Sevcan Ozel ${ }^{2}$, Seva Oner ${ }^{1}$, Leyla Uces $\overline{\text { Harmanogullarl }}^{1}$

${ }^{1}$ Department of Public Health, Mersin University Faculty of Medicine, Turkey ${ }^{2}$ Department of Public Health, Mersin University Enstitute of Health Science, Turkey

\section{Background}

Newborns should be admitted to the neonatal intensive care unit (NICU) for any reason, causing problems such as fear, sadness, guilt and anger on their families. Objective

To determine the reasons for stress of the families who were hospitalized baby in NICU.

\section{Methods}

This descriptive study's data was collected with questionnaire to 150 mothers who were infants in NICU. The questionnaire was included maternal and baby's some characteristics, the "Parental Stressor Scale: Neonatal Intensive Care Unit", and the "Nurse Parent Support Tool". Parental Stress Scale included; 6 items related to the sights and sounds in the NICU, 17 items about the baby's appearance and behaviors, and 11 items related to the parental role alteration. For each item on a 5 point Likert scale ranging from 1 (not stressful) to 5(extremely stressful). "Nurse Parent Support Tool" is Likert-type scale as follows: 1, almost never to 5 , almost always.

\section{Results}

The average age of mothers was $27.7 \pm 6.9$. Reasons for hospitalization; $27.3 \%$ were sepsis, $26.0 \%$ were respiratory complaints, $25.3 \%$ were prematurity, and $21.3 \%$ were other causes. According to the Parental Stressor Scale, the mothers were most likely to enter the stress in situations related to baby's appearance and behaviors (mean score $3.57 \pm 0.97$ ). This was followed by stress on the sights and sounds $(3.41 \pm 1.00)$ and the parental role $(3.36 \pm 0.85)$. The support given by the nurses to mothers was indicated "provide good care to her child, "be optimistic about her child" and "understand the special needs of her child".

\section{Conclusion}

It was determined that intensive care unit for mothers is stressful. Mother was stressed about the appearance and behavior of their babies. It is thought that the intensive care workers need to give more details about the characteristics of the intensive care environment and the appearance of their babies in order to reduce mother's stress.

\section{3}

\section{Critical/Emergency Care}

Pediatric snowboarding severe abdominal trauma: report of our experience

Nicola Zampieri ${ }^{2}$, Elia Trabucchi $^{1}$, Gabriele Trabucchi ${ }^{1}$, Carlo Romagnoli ${ }^{I}$

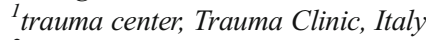

${ }^{2}$ Pediatric Surgical Unit, University of Verona, Italy

\section{INTRODUCTION}

Snowboarding injuries have increased and blunt abdominal trauma is frequent; the aim of this study is to report our experience with severe 
abdominal trauma treated in high mountain with a well skilled rescue team.

\section{MATERIALS AND METHODS}

We retrospectively review cases of severe abdominal trauma in snowboarder aged 6-18 years treated between December 2008 and December 2016. this specific high mountain skiarea has a well skilled reascue team with doctor. Demographic, type of injury, type of organ trauma, morbidity and outcomes were recorded and evaluated.

\section{RESULTS}

After reviewing casxes, 32 patients were enrolled. Thirty were male. 18 $(56 \%)$ cases had an associated head trauma with concussion and neurocognitive deficits; other 5 cases had head trauma without deficits. One patients had thoracic spine injury associated with complete spine injury. Twenty were injured in a fall, 10 collided with a stationary object and in 2 cases the mechanism of injury was unclear. Nineteen patients had single organ injury (18 spleen and 1 kidney); 5 patients had spleen and kidney, 6 patients liver and spleen and 2 patients had spleen, liver and pancreatic injury. Two patients undewent splenectomy and nephrectomy while only one patients had splenectomy alone. All the other were treated conservatively without morbidity. Younger patients 15 years were more likely to have multiple organ injuries (p0.05); all patients were secured and hospitalized by helicopter support. Mean time from injury to hospitalization was under 45 minutes.

\section{CONCLUSIONS}

Young male snowboarders are at risk for having multiple organ injuries; it is essential to hospitalyze these cases as soon as possible. Abdominal trauma especially after collision with stationary object are at risk for severe organ failure.

\section{5}

\section{Gastroenterology \& Nutrition}

\section{Awareness of ESPGHAN guidelines on coeliac disease amongst gen- eral paediatricians in Southwest England \\ Siba Prosad Paul ${ }^{1,3}$, Helen Adams ${ }^{2}$, Dharamveer Basude ${ }^{2,3}$ \\ ${ }^{T}$ Paediatrics, Torbay Hospital, UK \\ ${ }^{2}$ Medical School, University of Bristol, UK \\ ${ }^{3}$ Paediatric Gastroenterology, Bristol Royal Hospital for Children, UK}

\section{Background}

ESPGHAN 2012 guidelines on coeliac disease (CD) recommend that symptomatic children with anti-tissue transglutaminase titres (anti-tTG) 10x upper limit of normal (ULN), positive anti-endomysial antibody (EMA) results, and if HLA-DQ2/8 positive, can be diagnosed without a biopsy. However, non-biopsy diagnosis is not appropriate for: asymptomatic high-risk children (e.g. diabetes, first-degree relatives, Down's syndrome) and those with antitTG10xULN. Adequate knowledge of ESPGHAN guidelines is required by general paediatricians to ensure suspected $\mathrm{CD}$ patients undergo appropriate investigations for an accurate diagnosis.

\section{Aims}

1) To gain an understanding of awareness and use of ESPGHAN guidelines for diagnosing $\mathrm{CD}$ in children amongst general paediatricians

2) Provide recommendations to increase awareness if required.

\section{Methods}

A telephone/email survey was conducted of general paediatric consultants $(\mathrm{n} \approx 140)$ across Southwest England with 12 DGHs. Survey included 8 questions to assess awareness and use of ESPGHAN guidelines, incorporating 3 main themes: when non-biopsy diagnoses can be made, when HLA-DQ2/8 genotyping should be requested and whether asymptomatic children from high-risk groups with anti-tTG10xULN can be diagnosed without a biopsy.

\section{Results}

101/140 (72\%) responses obtained. Ninety-nine percent paediatricians were aware of ESPGHAN guidelines including non-biopsy/biopsy pathways for CD. Eighty-three percent paediatricians were unable to state all criteria required for non-biopsy diagnosis. None could describe all appropriate situations where HLA-DQ2/8 genotyping should be requested. Thirty-three percent paediatricians responded that asymptomatic children with anti-tTG10xULN can be diagnosed with CD without a biopsy while $24 \%$ said they would seek advice.

\section{Conclusions}

Survey highlighted need for greater in-depth awareness of non-biopsy pathway and situations where HLA-DQ2/8 genotyping is indicated. There is possible misinterpretation regarding the ESPGHAN guidelines as $1 / 3 \mathrm{rd}$ of paediatricians considered non-biopsy pathway is applicable for asymptomatic children with anti-tTG10xULN. There is need for improved understanding of the ESPGHAN guidelines amongst general paediatricians. A user friendly Apps is planned to improve the diagnostic process.

\section{4}

\section{Genetic \& Metabolic}

Counting birth defects in low and middle income country settings: The Sri Lanka's experience

Kapila Jayaratne ${ }^{I}$

${ }^{T}$ Ministry of Health, Family Health Bureau, Sri Lanka

\section{Background}

Availability of quality birth defects data, utilizing such data effectively at different levels and dissemination to all stakeholders, facilitate effective birth defects prevention and control.

\section{Objective}

We aimed to review opportunities available for integrating birth defects surveillance (BDS) into the existing healthcare delivery system and implement a BDS Methods

We conducted an in-depth analysis of healthcare system of Sri Lanka based on all sources available (documents/reports/internet searches/databases) to identify entry points and opportunities to integrate BDS.

Results

Sri Lanka reports a low maternal mortality ratio ( 32.5 per 100,000 live births) and infant mortality rate (9.7 per 1,000 live births). Birth defects contribute to $18.1 \%$ of infant deaths reported from hospitals. Prevalence of birth defects at birth $1.8 \%$. Antenatal care coverage (99\%) and institutional deliveries $(99.9 \%)$, a majority (92\%) in specialized units. Neonatal examinations (coverage $100 \%$ ) are carried out in all institutional births based on a standardised format. Perinatal deaths are subjected to institutional audits with a coverage of $98 \%$. Each house is designated to a public health midwife covering domiciliary post-partum care $(82 \%$ coverage).

A national birth defects prevention and control action plan was formulated and a national BDS mechanism was developed. BDS was implemented on pilot basis in Southern Province (10 hospitals) with the objective of scaling up to country-wide surveillance in coming years. Data are collected on to a web-based database maintained by Family Health Bureau. Preliminary data over one year has reported 419 cases up to 2 years. Leading causes were cardiac defects, chromosomal disorders, cleft lip/ palate and gastro-intestinal defects with female predominance. Many mothers were in $20-35$ year age group and a significant proportion $(28 \%)$ are 35 years.

\section{Conclusions}

With the available service delivery system, Sri Lanka and countries with similar contexts can easily integrate BDS as an effective measure to capture birth defects.

\section{1}

\section{Genetic \& Metabolic}

Discharging babies with Down Syndrome: improving compliance with guidelines

Carmel Maria Moore ${ }^{1}$, Salma Mohamed Eldaw ${ }^{1}$, Maeve McCormack ${ }^{1}$, John Fitzsimons ${ }^{I}$

${ }^{1}$ Department of Paediatrics, Our Lady of Lourdes Hospital, Ireland 
The Down Syndrome Medical Interest Group in UK and Ireland have guidelines suggesting safe standards of care for children and adolescents with Down Syndrome. We planned to create a checklist to improve compliance with these guidelines in a regional neonatal centre, and first audited historical compliance

\section{Method}

This retrospective review identified cases of babies with Down Syndrome from the Maternity Information System, in order to retrieve and review medical records. Anonymised data was analysed using a proforma.

Results

There were 20 babies diagnosed with Down Syndrome identified as part of the historical audit, all postnatally diagnosed with no antenatal concerns. Every baby had karyotype confirmation of their diagnosis of Down Syndrome, as well as referral to Early Intervention and Physiotherapy, TSH checked on Newborn Bloodspot Screening card and Newborn Hearing Screening. $90 \%(n=18)$ babies had cardiology referral before discharge. $35 \%(n=7)$ of babies were plotted on appropriate centile charts and only $25 \%$ of babies had ophthalmology referral completed. Early reports of checklist use $(n=2)$ demonstrated $100 \%$ compliance. Conclusions

Implementation of a checklist has the potential to improve compliance with guidelines for the medical discharge of babies with Down Syndrome.

564

Hemato - Oncology

Wilms tumor associated with genetic syndromes and congenital malformations

${ }_{\text {Ali Varan }}{ }^{\prime}$, Neslihan Kalkan, Bilgehan Yalcin, Burca Aydin, Nilgun

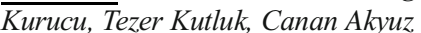

${ }^{1}$ Dept. of Pediatric Oncology, Hacettepe University, Cancer Institute, Turkey

\section{Aim}

To evaluate the presence of genetic anomaly, clinical malformation and syndromes with a tendency to develop Wilms tumor (WT).

Patients and Methods

Six hundred eighty-three patients with Wilms tumor who were treated between 1972 and 2016 were retrospectively analyzed. Clinical details, treatment and survival analysis were done. Associated congenital anomalies, genetic syndromes were noted.

Results

We found 54 patients with genetic anomaly, clinical malformation and syndromes with a tendency to develop tumors out of 683 patients with WT $(7 \%)$. Male/female ratio was 3.1. Mean age of diagnosis for WT was 3.3 years ( 0.18 years- 4 years). Syndromes with a tendency to develop tumors were Denys Drash $(n=10)$, Beckwith Wiedemann $(n=1)$, WAGR $(n=2)$, Fanconi $(n=1)$, Bloom $(n=1)$ and familial WT $(n=1)$. Genitourinary anomalies were horse shoe kidney $(n=6)$, hydrocele $(n=5)$, undescended testis $(n=11)$, hypospadias $(n=3)$, bilateral vesicoureteral reflux $(n=1)$, duplex ureter or collecting system $(n=1)$, renal hypoplasia $(n=1)$ and inguinal hernia $(n=4)$. Clinical malformations were hemihypertrophy $(n=8)$, isolated aniridia $(n=1)$ and other malformations $(n=2)$. We found 9 patients with bilateral WT. Thirty $(55.6 \%)$ patients received radiotherapy, 53 patients received chemotherapy $(98.1 \%)$ and 4 patients underwent partial nephrectomy. Three and five year overall survival rates were 74 and $63 \%$. Event-free survival rate was lower in female patients $(p=0.04)$.

Three out of 10 patients with Denys Drash syndrome are alive and 3 of them did not survive after 10 years due to secondary problems; the patient with Fanconi anemia died at the early period; the patients with Bloom and WAGR syndrome died in 11 and 18 years, respectively and the patient with vertebra anomaly died after 4.5 years due to syndrome related complications.

\section{Conclusion}

The most common associated anomalies were genitourinary anomalies and in long term follow up tumor unrelated deaths were more in syndromic patients.

\section{2}

\section{Infectious Diseases}

Beyond pneumonia: the importance of a complete clinical history.

Maria Loureiro $^{I}$, Miguel Costa ${ }^{I}$, Lucia Gomes ${ }^{I}$, Susana Tavares ${ }^{I}$

${ }^{7}$ Pediatrics, Centro Hospitalar Entre Douro e Vouga, Portugal

\section{Introduction}

Pulmonary tuberculosis (TB) still represents a serious public health problem. Young age and HIV infection constitute important risk factors for serious and systemic disease.

\section{Case-report}

We present the case of a 4-year-old boy with relevant priors of cerebral palsy, functionally totally dependent for activities of daily living. He was admitted for prolonged fever associated with a productive cough and dyspnea. He had been previously diagnosed with pneumonia and medicated with amoxicillin and clavulanic acid, having undergone therapy for 10 days, with no symptomatic improvement. When questioned, caretakers denied exposure to tuberculosis. Physical examination was compatible with septic shock in the context of pneumonia. X-ray supported the diagnostic hypothesis and showed a consolidation of the inferior lobe of the left lung. Therapy was instituted and the shock reversed. Afterwards, the patient had sustained fever and elevated values of sedimentation rate (SR), C-reactive protein (CRP) and transaminases. Antibiotics were escalated to ceftriaxone, clindamycin and vancomycin. There was no clinical improvement. The patient remained feverish and had radiological worsening on chest $\mathrm{x}$-ray performed at the 13th day of internment. Chest tomography showed a miliary pattern on both lobes of the left lung. Mycobacterium tuberculosis was isolated on gastric aspirate. When confronted, caretakers finally admitted that there had previously been a member of the household infected with tuberculosis, but didn't consider this as exposure "because the child was bedridden" (sic). Antitubercular and corticotherapy were instated, with significant clinical improvement.

\section{Conclusion}

TB in pediatric age continues to be a diagnostic challenge, especially when clinical presentation is atypical. Clinicians must be aware of the symptoms in order to make a prompt diagnosis, allowing early institution of proper therapy, decisive for prognosis.

798

\section{Infectious Diseases}

Demographic, Clinical and Laboratory Characteristics of Acute Rotaviral Gastroenteritis in Children Admitted to Department of Infectious Diseases, University Medical Centre Ljubljana from 2015 to 2016 Živa Makovec ${ }^{1}$, Gregor Verček ${ }^{l}$, Lucijan Kobal ${ }^{1}$, Breda Zakotnik², Stefan Grosek $^{1,3}$

${ }^{1}$ Department of Paediatric Surgery and Intensive Care, University Medical Centre Ljubljana, Slovenia

${ }^{2}$ Department of Infectious Diseases, University Medical Centre Ljubljana, Slovenia

${ }^{3}$ Department of Paediatrics, Medical Faculty, University of Ljubljana, Slovenia

\section{Background}

Rotavirus is the most common cause of hospitalization for acute gastroenteritis in children and still has a high mortality rate in the developing world.

\section{Objective}

To analyze demographic, clinical and laboratory characteristics at time of admission, and fluid replacement therapy among children with acute rotaviral gastroenteritis, admitted to Department of Infectious Diseases. 


\section{Methods}

We reviewed medical records of hospitalized patients under the age of 18 with the International Classification of Diseases diagnosis of rotaviral gastroenteritis (ICD A080) between September 1st 2015 and August 31st 2016.

\section{Results}

Two hundred and seven patients, median age 21 months, 194/207 (94\%) under the age of 7, 97 (47\%) girls, were hospitalized for a median length of stay 2 days. The majority of patients were healthy before the onset of acute gastroenteritis. Vomiting and diarrhea were found in 164/207 (79 \%), only vomiting in 32/207 (15\%), and diarrhea in 11/207 (5\%), beginning two days (median value) before admission. Forty-five percent were afebrile. Eleven patients $(5 \%)$ were admitted with another or concomitant diagnosis. No dehydration severity scoring systems were used to grade the severity of dehydration, the clinicians rather used a combination of one or more clinical signs or symptoms to assess the severity of dehydration, most frequently general appearance, moisture of mucus membranes, heart rate, urine output, skin elasticity and capillary refill time. Ninety-eight $(46 \%)$ had elevated CRP, $28(13 \%)$ were hyponatremic, none was hypernatremic. In almost all of the cases, intravenous infusion was started immediately, with average total $84 \mathrm{ml} /$ $\mathrm{kg}$ of body mass received during hospitalization. Readmission rate was low $(6 / 207,2.9 \%)$. No patients died.

\section{Conclusion}

Early admission after the onset and early beginning of the fluid replacement therapy were found among our patient. There were only few cases of severe dehydration or severe electrolyte imbalances.

\section{5}

\section{Infectious Diseases}

Severity of lower respiratory tract infections in school-age children is associated with Mycoplasma pneumoniae genotype

Jasna Rodman ${ }^{1}$, Darja Keše ${ }^{2}$, Marina Praprotnik ${ }^{l}$, Tatjana Mrvič ${ }^{3}$, Rok Kogoj', Uroš Krivec ${ }^{1}$

${ }^{1}$ Unit for Pulmonary Diseases, University Children's Hospital, University Medical Centre Ljubljana, Slovenia

${ }^{2}$ Institute of Microbiology and Immunology, Faculty of Medicine, University of Ljubljana, Slovenia

${ }^{3}$ Department of Infectious Diseases, University Medical Centre Ljubljana, Slovenia

\section{Background}

Mycoplasma pneumoniae (MP) can be categorized into two genotypes, P1 type 1 (MP1) and P1 type 2 (MP2), based on the DNA sequence of the $\mathrm{P} 1$ adhesion protein. It remains unknown if clinical manifestations of lower respiratory tract infections (LRTI) in children differ between the two genotypes.

Objective

To determine if MP genotype is associated with disease severity of LRTI in preschool and school-age children.

\section{Methods}

Medical charts of children ( $\leq 15$ years-old) with signs of LRTI who tested positive for MP by PCR from pharyngeal swabs in 2014 were analysed. We used a culture and pyrosequencing approach for genotyping PCRpositive samples. We compared epidemiological and clinical data of children infected with either MP1 or MP2 divided in two age groups, $\leq$ 5 year-olds and $5-15$ year-olds.

Results

Among 5-15 year-olds, 208 patients were infected with MP1 (mean age $8.6 \mathrm{yrs}, 46 \%$ boys) and 69 with MP2 (mean age $8.3 \mathrm{yrs}, 59 \%$ boys). MP2 infected children presented with higher median baseline CRP level (16 vs $28 \mathrm{mg} / \mathrm{L}, p=.006)$ and were admitted to the hospital more often $(22.6 \%$ vs $34.8 \%, p=0.045)$. There was no difference in the requirement for oxygen treatment $(40.4 \%$ vs. $33.3 \%)$ or in the average duration of hospital stay ( 2.7 days vs 2.3 days) between hospitalised patients. No intensive care treatment was required in either group. No group differences in clinical data were observed among $\leq 5$ year-olds.

\section{Conclusion}

In contrast to previous smaller studies, the data from our large cohort indicate that MP genotype could influence the severity of LRTI in school-age children. Patients infected with MP2 in this age group presented with a higher median baseline CRP level and a higher rate of hospital admission.

741

Infectious Diseases

The Where and the Why of Intravenous Antibiotic Treatment for Febrile Urinary Tract Infection/Pyelonephritis in Children

Barry Scanlan $^{1,2,3,4}$, Laila Ibrahim ${ }^{1,2,3}$, Sandy Hopper ${ }^{1,2}$, Franz Babl $^{1,2,3}$, $\overline{\text { Andrew Davidson }}^{1,2,3}$, Penelope Bryant ${ }^{1,2,3}$

${ }^{1}$ Paediatrics, The Royal Children's Hospital, Australia

${ }^{2}$ Clinical Paediatrics, The Murdoch Children's Research Institute, Australia

${ }^{3}$ Paediatrics, Univerisity of Melbourne, Australia

${ }^{4}$ Paediatrics, The Royal College of Physicians of Ireland, Ireland

\section{INTRODUCTION}

Febrile UTI/pyelonephritis is a common diagnosis for children presenting to the Emergency Department(ED). A Cochrane review of the management of pyelonephritis in children showed no difference between intravenous (IV) and oral antibiotics. Despite this, many children are treated with at least initial IV antibiotics. The reasons are often unclear and it is uncertain whether this is appropriate as the Cochrane review excluded children at the worse end of the clinical spectrum. Our aim was to determine why physicians made these decisions as a first step in determining whether this is necessary.

\section{METHODS}

A prospective observational study of children presenting to the ED at the Royal Children's Hospital with UTI/pyelonephritis from Oct.2016Apr.2017. Data collection included demographic, clinical features, microbiology, treatment and outcomes. ED physicians were asked to record reasons for prescribing IV antibiotics and for treating the patient in hospital versus in hospital-in-the-home(HITH).

\section{RESULTS}

One hundred seventy children were included, 123(72\%) were treated with oral antibiotics, 43(25\%) IV and 4(3\%) intramuscular - these are now included in the IV group. In the IV group, 32(19\%) were admitted, 3(2\%) were transferred directly to HITH from ED and 13 had a single dose of IV antibiotics in $\mathrm{ED}$ and were discharged. A comparison of clinical features between the two groups is outlined in the table. The commonest reasons cited for using IV antibiotics and for hospital admission versus HITH are shown in the figures.

\section{CONCLUSIONS}

This is the first study of decision-making for IV antibiotics and hospital admission for UTI/pyelonephritis in children. The fact that the reasons cited for IV antibiotics were often not evident on objective clinical data suggests that there may be overuse of IV antibiotics. We will present an assessment of appropriateness and use the results to suggest alternatives to the traditional IV and hospital admission route for these children.

\begin{tabular}{|c|c|c|c|}
\hline & $\begin{array}{c}\text { Oral } \\
\text { Patient no. (\%) } \\
n=123\end{array}$ & $\begin{array}{c}\text { Intravenous } \\
\text { Patient no. (\%) } \\
n=47\end{array}$ & $p$ value \\
\hline Age(yr), mean (range) & $4.8(0.3-17.9)$ & $5.4(0.3-16.5)$ & 0.68 \\
\hline Sex, Female: Male & $3: 1$ & $2.9: 1$ & 1 \\
\hline \multicolumn{4}{|l|}{ At presentation } \\
\hline Prior antibiotics* & $14(11 \%)$ & $12(26 \%)$ & 0.03 \\
\hline Previous UTI & $32(26 \%)$ & $17(36 \%)$ & 0.26 \\
\hline $\begin{array}{l}\text { History of resistant } \\
\text { organism* }\end{array}$ & $7(6 \%)$ & $9(19 \%)$ & 0.02 \\
\hline \multicolumn{4}{|l|}{ Duration of Symptoms } \\
\hline$<24$ hrs & $39(31 \%)$ & $13(28 \%)$ & 0.71 \\
\hline$>48 \mathrm{hrs}$ & $44(36 \%)$ & $23(49 \%)$ & 0.16 \\
\hline \multicolumn{4}{|l|}{ Symptoms } \\
\hline Fever* & $63(51 \%)$ & $36(77 \%)$ & $<0.01$ \\
\hline Rigors* & $7(6 \%)$ & $9(19 \%)$ & 0.02 \\
\hline Vomiting & $38(31 \%)$ & $18(38 \%)$ & 037 \\
\hline Lethargy & $25(20 \%)$ & $11(23 \%)$ & 0.68 \\
\hline
\end{tabular}




\section{Reason for i.v. antibiotics}

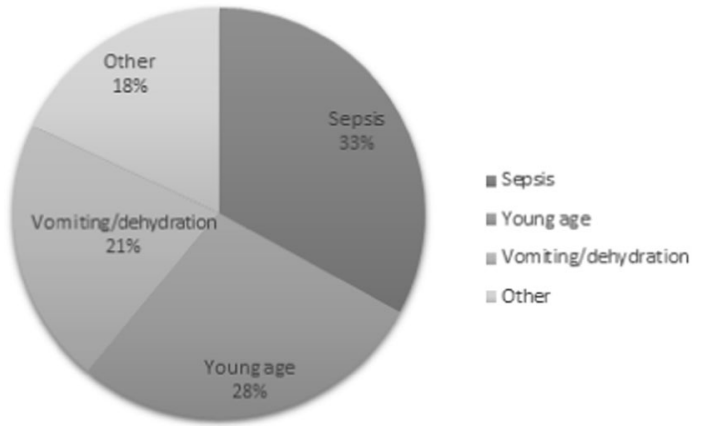

Reason for hospital admission versus HITH

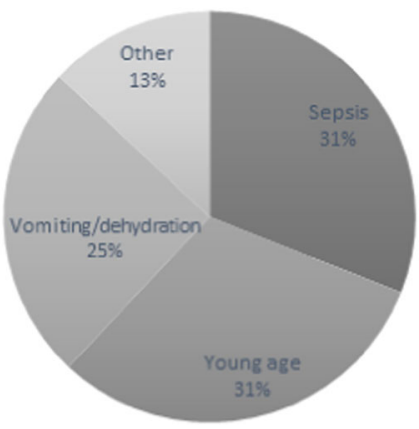

- Sepsis

Y Young age

W Vomiting/dehydration

॥ Other

690

\section{Infectious Diseases}

A systematic review about S.pneumoniae serotype distribution in children in mainland of China

Shuang Lye ${ }^{1}$, Hui-li Hu ${ }^{1}$, Kaihu Yao ${ }^{1}$, Yong-hong Yang ${ }^{1}$

${ }^{1}$ Pediatric Research Institute, Beijing Children's Hospital, China

\section{Aim}

To evaluate the serotype distribution of S.pneumoniae in China, from which multi-centers survies were limited.

\section{Methods}

This review systematically reviewed the studies conducted among 2006 to 2015 , with serotype data of S.pneumoniae isolates among children in China hinterland from the published literatures. Using Median (M) and Decile Range (D) to analyse the serotype proportion andl vaccine coverage rate.

\section{Results}

A total of 35 papers were collected for anlysis. Among non-invasive strains, the rates of serotypes were $19 \mathrm{~F}(28.2 \%), 23 \mathrm{~F}(12.2 \%), 19 \mathrm{~A}$ $(16.4 \%), 14(8.0 \%)$, and 6B (4.9\%) in north region; and in south region, the rates of serotypes were $19 \mathrm{~F}(44.6 \%), 23 \mathrm{~F}(9.2 \%), 19 \mathrm{~A}(13.3 \%), 6 \mathrm{~B}$ $(8.1 \%)$, and $14(6.0 \%)$. Among invasive strains, the rates of serotypes were 19A (23.8 \%), $19 \mathrm{~F}(16.0 \%), 14(17.2 \%), 23 \mathrm{~F}(8.2 \%)$, and 6B $(4.8 \%)$ in north region; and in south region, the rates of serotypes were $19 \mathrm{~F}(24.5 \%), 19 \mathrm{~A}(19.6 \%), 14(18.2 \%), 6 \mathrm{~B}(6.6 \%)$, and $23 \mathrm{~F}(8.7 \%)$; while in multicenter, the rates of serotypes were $19 \mathrm{~F}[21.2 \%$ (D: $17.7 \%$ $27.0 \%$ )], 19A [23.5 \% (D: $11.6 \%-32.0 \%$ )], $23 \mathrm{~F}$ [4.8 \% (D:2.5\%$7.7 \%$ )], 14 [9.8\% (D: $5.9 \%$ - 17.4)].

\section{Conclusions}

In hinterland of China, the prevailing serotypes in children under 14 years old were serotype $19 \mathrm{~F}, 19 \mathrm{~A}, 23 \mathrm{~F}, 14$ and 6B. The serotype coverage rate of PCV13 was higher than PCV10 due to the high rate of serotype 19A.
194

Neonatology

Fetal Arrythmias. Before and after birth. A Leeds experience from 2000-2012

Jawad Ahmed Abbasi ${ }^{l}$, Elspeth Brown ${ }^{1}$

${ }^{T}$ Paediatric Cardiology, Leeds General Infirmary, UK

\section{Objective}

To review the management of fetal arrhythmias during pregnancy and follow these fetuses in later life to monitor final outcome.

\section{Method}

Retrospective review on a database of 139 pregnant women and follow up of their babies in cases here details were available. Arrhythmias were grouped together based on their type. Antenatal management of arrhythmias and progress of babies into later childhood was documented.

\section{Results}

Patients were subclassified into heart blocks (n27, live birth n17), atrial flutter (n7, live birth n6), supraventricular tachycardia (SVT) (n38, live birth n15), ectopics (30), arrhythmia with abnormal heart structure (17), and others (18). No antenatal treatment was used to improve heart rate in bradyarrhythmia and postnatal treatment was an artificial pace maker in majority of live births at some stage of childhood. In tachyarrhythmias the rate was controlled with digoxin or flecanide and amiodarone was used in refractory cases. A tachyarrhythmia may resolve spontaneously after birth but may need a combination of anti arrhythmics to control rate. If a baby has an SVT in neonatal period than he is more likely to require radio frequency ablation later in life as compared to the one's who do not have SVT in neonatal period. These babies can be symptom free and come off anti arrhythmics in early years of life.

\section{Conclusion}

In case of a live birth with heart block, majority of babies will need a pacemaker at some stage of their life with good quality of life provided that they do not have complications of pacemaker insertion. Tachyarrhythmias can be managed with antiarrhythmic therapy in utero and have a favorable outcome later on in life provided the rate can be controlled in utero.

\section{7}

\section{Neonatology}

Quality of Life of Children Born Extremely Preterm at School-Age

Veronique Brevaut-Malaty ${ }^{I}$, Catherine Gire ${ }^{1,2}$, Noemie Resseguier ${ }^{2}$, Julie Berbis $^{2}$, Stéphane Marret ${ }^{3}$, Gilles Cambonie ${ }^{4}$, Jean-Baptiste Muller ${ }^{5}$, Isabelle Soudski-Medioni ${ }^{6}$, Patricia Garcia ${ }^{7}$, Pascal Auquier ${ }^{2}$

${ }^{I}$ Neonatal Intensive Care Unit, Hôpital Nord, Assistance Publique Hôpitaux Marseille, France

${ }^{2}$ EA3279 Qualité de Vie et Maladie Métabolique, Aix Marseille Université, France

${ }^{3}$ Neonatal Intensive Care Unit, Centre Hospitalo-Universitaire Rouen, France

${ }^{4}$ Neonatal Intensive Care Unit, Hôpital Arnaud de Villeneuve, France

${ }^{5}$ Neonatal Intensive Care Unit, Centre Hospitalo-Universitaire Nantes, France

${ }^{6}$ Neonatal Intensive Care Unit, Hôpital de Nimes, France

${ }^{7}$ Neonatal Intensive Care Unit, Hôpital Conception, Assistance Publique - Hôpitaux Marseille, France

\section{Background}

Major disability handicaps amongst extremely premature birth (EPB) children is on the decline. To date, no studies evaluate the quality of life (QoL) of these school-aged children. 


\section{Objective}

To determine the QoL of school-age children, born before 28 weeks gestation (GA), that have no major disability.

\section{Methods}

A cross-sectional, six-center study of children born at GA 28 weeks, between 01/12/2004 and 31/12/2007; discharged alive; and without severe morbidity (cerebral palsy, autism, and severe mental retardation). These children, between 7 and 10 years old, had clinical examinations and cognitive function and $\mathrm{QoL}$ assessments using validated standardized questionnaires which were completed by both children and parents. QoL measurements, using 2 generic self- and hetero-evaluation questionnaires (Kidscreen-10/VSPA), were compared to French population norms.

\section{Results}

Of the 471 children included, $302(64 \%)$ had a mean GA of $26.2( \pm 0.8)$ weeks; a mean birth weight of $879( \pm 181)$ grams; $140(30 \%)$ were lost to follow-up (LFU); and 29 (6\%) were lost due to parental refusal. Socioeconomic characteristics of the LFU and refusal groups were significantly different from those children within the study. The mean assessment age was $8.4( \pm 0.87)$ years. Of the 250 children having a neuro-psychological assessment, $71(28.4 \%)$ had minor sequelae, $49(19.6 \%)$ had moderate sequelae and $130(52.0 \%)$ had no sequelae. The QoL $(n=302)$ was significantly lower than the reference population, regardless of the questionnaire used (Kidscreen 10/VSPA), in all areas, except the children's assessment of family relationships. Perceptions within the "parent-child" couple, as compared to the reference population, showed parents underestimated the school domain QoL, and children underestimated the QoL with friends.

Conclusion

The QoL of the EPB population without severe sequelae is low compared to the reference population.

305

\section{Neonatology}

Hypothermia is Neuroprotective in Severe Hypoxic-Ischaemic Injury, With Infection as Co-morbidity

Mari Falck ${ }^{l}$, Damjan Osredkar ${ }^{1,2}$, Elke Maes ${ }^{l}$, Torun Flatebo ${ }^{l}$, Thomas Wood $^{I}$, Hemmen Sabir ${ }^{1,3}$, Marianne Thoresen ${ }^{1,4}$

${ }^{I}$ Department of Physiology, University of Oslo, Institute of Basic Medical Sciences, Norway

${ }^{2}$ Department of Paediatric Neurology, University Children's Hospital, Slovenia ${ }^{3}$ Department of General Paediatrics, Neonatology and Paediatric Cardiology, University Children's Hospital, Heinrich-Heine University, Germany

${ }^{4}$ School of Clinical Sciences, University of Bristol, UK

\section{Background}

Perinatal infections increase the neonatal brain's vulnerability to hypoxicischemic (HI) injury. Systemic inflammation induced through TLR-4 (LPS), negates hypothermic neuroprotection in a model of moderate brain injury. ${ }^{1}$ We recently showed that after inflammatory pre-sensitisation induced through TLR-2, mimicking a Gram-positive infection, therapeutic hypothermia (TH) provided $80 \%$ neuroprotection. ${ }^{2}$ HT has been shown not to be effective in severe brain injury ${ }^{3}$, still we treat babies with severe neurological dysfunction with HT. Whether HT is neuroprotective in severe brain injury with inflammatory pre-sensitisation is unknown.

\section{Objective}

To examine the effect of TH after severe unilateral neonatal brain injury, with inflammatory pre-sensitisation with a TLR2 agonist $\left(\mathrm{PAM}^{3} \mathrm{CSK}^{4}\right)$.

Methods

One hundred ninety-six seven-day-old (P7) Wistar rats received $0.9 \%$ $\mathrm{NaCl}$ (Veh) or $\mathrm{PAM}^{3} \mathrm{CSK}^{4}(1 \mathrm{mg} / \mathrm{kg}$ ), intraperitoneally. After an 8-hour delay, the left carotid artery was ligated followed by hypoxia $\%$ O2. Pups were randomized to $5 \mathrm{~h}$ of normothermia ${ }_{37{ }^{\circ} \mathrm{C}}$ or $\mathrm{TH}_{32^{\circ} \mathrm{C}}$ after the insult. Hemispheric $(\mathrm{He})$ and hippocampal (Hi) area loss analyses was performed at P14.

\section{Results}

Normothermic $\mathrm{PAM}^{3} \mathrm{CSK}^{4}$-animals (PAM-NT) had similar median area loss (He: $60 \%$ (33-66); Hi: $61 \%$ (29-67) as Veh-animals (Veh-NT) (He: $58 \%$ (11-64); Hi: $60 \%$ (19-68)), defined as severe brain injury. TH significantly reduced hemispheric injury in control groups $(p=0.048)$ and in $\mathrm{PAM}^{3} \mathrm{CSK}^{4}$-animals $(p=0.03$ ) (Fig 1). Hypothermia was also neuroprotective at the hippocampal level (Veh-HT vs Veh-NT: $p=0.042$; PAM-HT vs PAM-NT: $p=0.027$ ) (Fig 2).

\section{Conclusion}

In this double hit model of severe brain injury with inflammatory presensitisation through TLR-2, TH significantly reduced injury in $\mathrm{PAM}^{3} \mathrm{CSK}^{4}$-animals and in Veh-animals. These findings indicates that the question of hypothermic neuroprotection in case of severe injury is not finally determined, and furthermore, that a gram-positive infection would not negate the therapeutic effect of HT at any degree of brain injury.

- Osredkar, D. et al. Resuscitation 85, 567-572 (2014)

- Falck, M. et al. http://www.abstracts 2 view.com/pas/ view.php?nu = PAS16L1_4615.4 (2016)

- Sabir, H. et al., Stroke 43, 364-370 (2012)
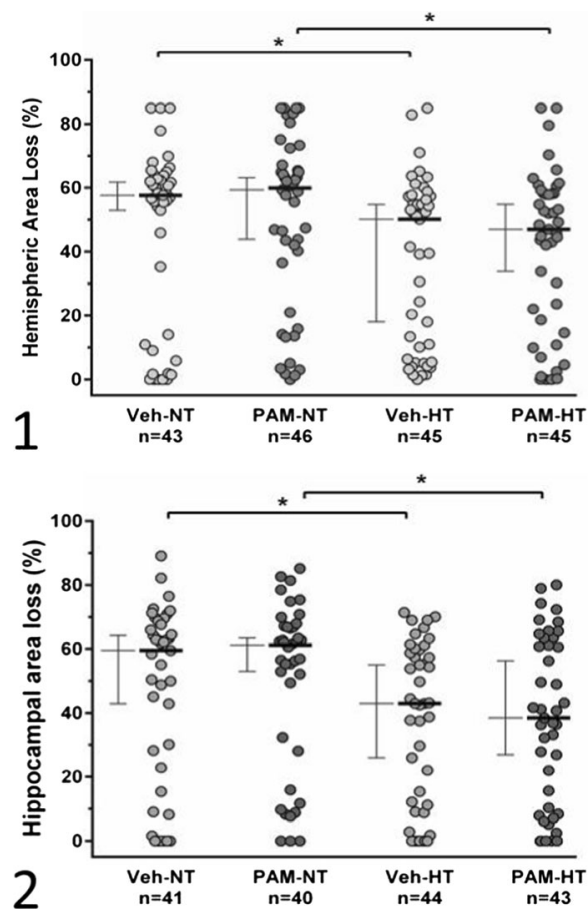

416

Neonatology

Cardiorespiratory Parameters in Newborns During Sedation with Chloral Hydrate

$\underline{\text { Petja Fister }}^{l}$, Jerneja Peček ${ }^{l}$, Barbara Gnidovec Stražišar ${ }^{2}$, Darja Paro Panjan ${ }^{I}$

${ }^{1}$ Division of Paediatrics, Department of Neonatology, University Medical Centre Ljubljana, Slovenia

${ }^{2}$ Department of Paediatics, General Hospital Celje, Slovenia

\section{BACKGROUND}

We commonly use chloral hydrate sedation in newbornsto facilitate painless diagnostic procedures, though its cardiorespiratory side effects have not yet been fully investigated. Previous pediatric studies were mostly retrospective, based on intermittent 
measuring of vital signs and have mainly included older infants and children.

\section{OBJECTIVE}

Our study used continuous recording of cardiorespiratory functions by polygraphy, including end-tidal $\mathrm{CO}_{2}$ monitoring, with the aim to analyze the impact of chloral hydrate on cardiorespiratory parameters in term newborns.

\section{METHODS}

We performed a prospective, pre-post single-arm interventional study in 42 term respiratorily and hemodynamically stable newborns. Oxygen saturation $\left(\mathrm{SpO}_{2}\right)$, end-tidal $\mathrm{CO}_{2}\left(\mathrm{ETCO}_{2}\right)$, apneahypopnea index and respiratory and heart rate were recorded with polygraphy, starting $0.5-1$ hour before the administration of chloral hydrate at a dose of $40 \mathrm{mg} / \mathrm{kg}$ and stopping 4 hours postadministration.

\section{RESULTS}

All measured cardiorespiratory parameters changed significantly after administration of chloral hydrate. The mean basal $\mathrm{SpO}_{2}$ dropped by $2.0 \%$ (from $97.1 \%$ to $95.1 \%$; p.001) and the mean basal ETCO2 increased by $3.9 \mathrm{mmHg}$ (from 25.6 to $29.5 \mathrm{mmHg}$; p.001). We also found a significant decrease in minimal $\mathrm{SpO}_{2}$ values (p.001) and an increase in the proportion of time spent with $\mathrm{SpO}_{2} 95 \%$ and $90 \%$ (p.001). The mean increase of estimated apnea-hypopnea index was 3.5 events per hour (p.001). The mean respiratory and heart rate were significantly lower $150 \mathrm{~min}$ after administration of chloral hydrate when compared with pre-sedation values $(51 / \mathrm{min}$ and $127 / \mathrm{min}$ versus $61 / \mathrm{min}$ and $138 / \mathrm{min}$ respectively; p.001).

\section{CONCLUSIONS}

Chloral hydrate sedation at a dose of $40 \mathrm{mg} / \mathrm{kg}$ causes mild changes in $\mathrm{SpO}_{2}, \mathrm{ETCO}_{2}$, estimated apnea-hypopnea index and respiratory and heart rate. These cardiorespiratory parameters remained within normal limits in most newborns, confirming the safety of chloral hydrate sedation. Nevertheless, since cardiorespiratory effects of chloral hydrate varied considerably among newborns, monitoring of sedated newborns continues to remain necessary.

\section{0}

\section{Neonatology}

Pharmacoepidemiology of antifungal drugs in 16 French neonatal intensive care units

Jean-Bernard Gouyon ${ }^{1}$, Severine MARTIN ${ }^{1}$, Nastasia Fouret ${ }^{1}$, Silvia Iacobelli ${ }^{I}$, Béatrice Gouyon ${ }^{1}$

${ }^{1}$ CEPOI Centre d'Etudes Périnatales de l'Océan Indien, CHU de La Réunion, Reunion

\section{Background}

Invasive candida infection is a devastating condition amongst very and extremely preterm infants cared for in NICUs. Fluconazole (FC) is the most widely used antifungal medication in neonatology for both prophylactic and curative treatment of candida infections (invasive or local). Point prevalence surveys have shown that FC is frequently underdosed in NICUs (Lestner 2015 Antmicrob., Chemother, 2015) a condition favouring a loss of efficacy and increased mortality.

\section{Objective}

We hypothezised that a computerized prescribing order entry (C.P.O.E.) system could allow preselected prescription of antifungal drugs and should make doctors more compliant with professional recommendations even though the prescriber always has the final decision.

Methods

Sixteen NICUs used the same C.P.O.E. system for all neonatal prescriptions. All prescriptions were made anonymous monthly and exported in a common database.

\section{Results}

Population : 13135 newborns : 998 were administered antifungal drugs. Overall, 273 patients were given a curative antifungal medication for invasive infection (57 after prophylaxis and 5 after an orodigestive infection) ; 588 were given prophylaxis (FC or nystatin) ; 194 were given orodigestive/local treament. Table shows that the lower the GA the higher the rate of antifungal treatment.

Fluconazole was more prescribed $(59 \%)$ than Nystatin, the different forms of Amphotericin B, caspofungine, flucytosine, mycafungine and miconazole. Amongst the 350 patients administered prophylactic Fluconazole the rate of underdosage was $0 \%$ and the rate of overdosage was $7.4 \%$.

\section{Conclusion}

This pharmaco- epidemiological study shows the possibility of assisting prescribers in the choice of the right doses in the case of antifungal drugs.

\section{5}

\section{Neonatology}

Cyclodextrine given intravenously after severe neonatal hypoxia, - alone or in combination with progesterone, increased autophagy activation and reduced endoplasmatic reticulum stress in prefrontal cortex

Rønnaug Solberg ${ }^{1,2}$, Silvia Carloni ${ }^{4}$, Serafina Perone ${ }^{3}$, Mariangela Longini $^{3}$, Fabrizio Proietti ${ }^{3}$, Håvard Tetlie Garberg ${ }^{1}$, Marianne Ullestad Huun ${ }^{1}$, Giulia Riparini ${ }^{4}$, Ola Didrik Saugstad ${ }^{l}$, Giuseppe Buonocore ${ }^{3}$, Walter Balduini ${ }^{4}$

${ }^{1}$ Department of Pediatric Research and Surgical Research, University of Oslo and Oslo University Hospital, Norway

${ }^{2}$ Department of Pediatrics, Vestfold Hospital Trust, Norway

${ }^{3}$ Pediatric Neonatology Unit, University Hospital of Siena, Italy

${ }^{4}$ Department of Biomolecular Sciences, University of Urbino "Carlo Bo", Italy

\section{Background}

Perinatal hypoxic-ischemic brain damage is a major cause of acute mortality and chronic neurologic morbidity in infants and children. Progesterone dissolved in the vehicle Cyclodextrine has shown promising results if given after traumatic brain injury. Endoplasmatic reticulum (ER) pathology may be an indicator of the severity of neuronal injuries. Therapies to attenuate ER stress applied at delayed stages, may rescue stressed neurons after neonatal hypoxia-ischemia. Together with measurements of autophagy activation, it can be used to determine effects of neuroprotective treatments.

\section{Objective}

The aim was to explore if cyclodextrine given i.v. after severe perinatal hypoxia had an equal neuroprotective effect as progesterone dissolved in cyclodextrine.

\section{Methods}

Severe global hypoxia was induced in newborn piglets. Reoxygenation with ambient air, one group $(N=12)$ got $8 \mathrm{mg} / \mathrm{kg}$ progesteron dissolved in the vehicle 2hydroxypropyl- $\beta$-cyclodextrin i.v., another group $(N=10)$ got $2 \mathrm{ml} / \mathrm{kg}$ vehicle(Cyclodextrine) i.v. and a reference group just received $2 \mathrm{ml} / \mathrm{kg}$ saline i.v. $(N=11)$. The piglets were observed for $9.5 \mathrm{~h}$ after end hypoxia. Brain tissues from prefrontal cortex were sampled and the effects of progesterone and the vehicle cyclodextrine on ER stress and autophagy were measured. We assessed the molecular chaperons GRP78 and Hsp70 for ER stress and for autophagy activation of the expression of $\mathrm{LC} 3$ (a microtubule-associated protein) and p62 in cortical tissue. 


\section{Results}

The expression of GRP78 and Hsp70 were significantly reduced in both treatment groups indicating reduced ER stress. The expression of Lipidated LC3 was significantly increased and the autophagy activation confirmed by a significant reduction of p62 expression. The results are in line with reported effects of neuroptotective treatments.

\section{Conclusions}

Treatment with Progesterone dissolved in Cyclodextrine or Cyclodextrine alone after severe neonatal asphyxia increased autophagy and reduced endoplasmatic reticulum stress. The results highlight the independent therapeutic effects of a commonly used vehicle for neuroprotective drugs.

519

\section{Neonatology}

Aerosolized Survanta in Neonatal Respiratory Distress Syndrome: Phase I Study Beena Sood ${ }^{l}$, Madhuri Kolli ${ }^{I}$, Josef Cortez ${ }^{1}$, Virginia Delaney-Black ${ }^{1}$

${ }^{T}$ Pediatrics, Wayne State University, USA

\section{BACKGROUND}

Respiratory distress syndrome (RDS) is the leading cause of mortality and morbidity in preterm infants (PTI). Intratracheal instillation, the only approved means of surfactant delivery, requires endotracheal intubation and mechanical ventilation with their attendant risks. Widespread use of non-invasive ventilation (NIV) for initial respiratory support of PTI not only averts the need for intubation and MV but also delays surfactant instillation. We hypothesized that treatment of RDS with aerosolized surfactant (AS) in PTI undergoing NIV is safe and feasible.

\section{METHODS}

We initiated a Phase I study of AS in PTI37 weeks GA with RDS undergoing NIV. Infants with pneumothorax, lethal malformations, death anticipated within 72 hours, and those requiring immediate intubation were excluded. Infants were stratified by gestational age (GA) and sequentially enrolled infants received increasing doses and dilutions of surfactant using a MiniHeart jet nebulizer. Infants were monitored during AS and for 72 hours after AS.

\section{RESULTS}

Of 17 patients enrolled, 15 were African American and 8 males. Two extremely PTI exited the study for worsening respiratory distress after one dose of AS. Fifteen infants completed study intervention; two were weaned to room air after the 1 st dose of AS and 13 received 2 doses. Infants tolerated the aerosol treatment well. No significant adverse events were identified. Of 15 infants that completed the study, one required medical treatment for a patent ductus arteriosus; one received vasopressors, two were treated medically for necrotizing enterocolitis (NEC) and one was intubated at 19 days of age following a diagnosis of NEC. Majority of the 17 enrolled infants were discharged to home $(n=15)$ without respiratory support $(n=14)$ at a mean corrected GA of $35^{5 / 7}$ weeks.

\section{CONCLUSIONS}

We have demonstrated the feasibility of administering AS to PTI; AS was well tolerated by infants and clinical caregivers.

714

Neonatology

Does the nociceptive spinal reflex explain the lack of correlation between the observational and physiological pain assessment scores in neonates? Hanne Storm ${ }^{I}$

${ }^{T}$ Acute clinic, Rikshospitalet University hospital, University of Oslo, Norway

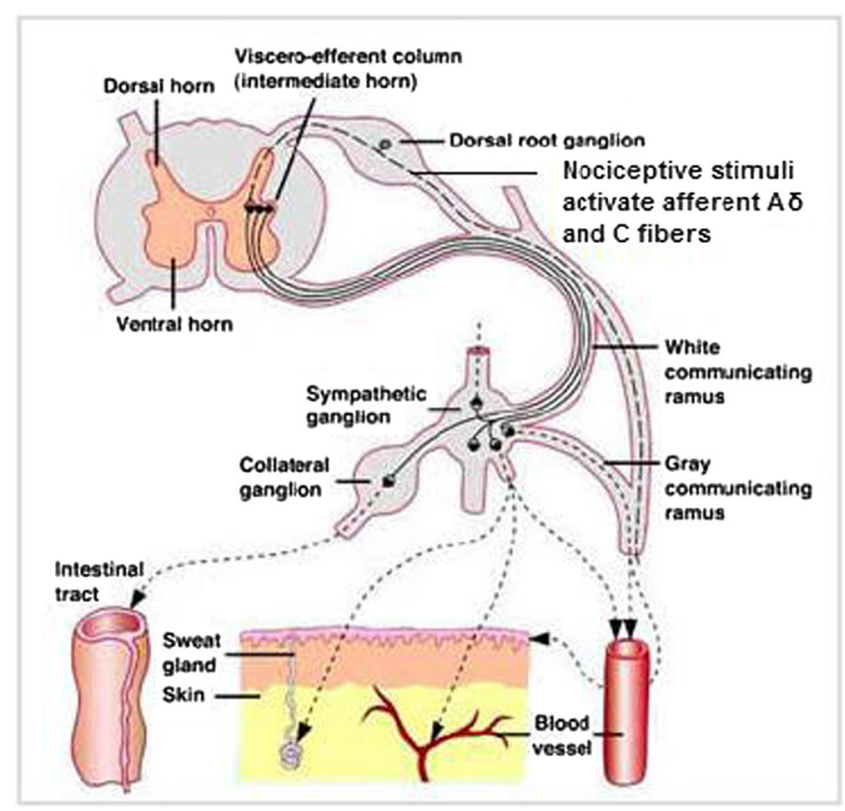

The nociceptive spinal reflex.

\section{Background}

In the US it is mandatory to assess and treat pain. UK, The Netherlands, Italy, France and Russia are also establishing similar guidelines. Physiological, behavioural, and biochemical tools have been used for pain assessment in neonates but no gold standard is yet available. Objectives To examine why the findings from behavioural and physiological responses during painful responses have been reported to be either uncorrelated or weakly correlated (Stevens et al. 1994; Nurs Res, 43, 226-231, Johnston et al. 1995;Pain, 61, 471-9, Barr et al. 1998; Arch Dis Child Fetal Neonatal Ed, 79, 152-56).

Methods

To theoretical compare the anatomical nociceptive spinal reflex to both the behavioural pain scores and the physiological pain assessment tools to understand why these pain assessment methods are uncorrelated. The reasons may be multiple; the caregiver may interpret the behavioural responses differently, and the behavioural responses may be less reactive in very ill patients, small-forgestational age infants, and different for infants who have been exposed to severe and repetitive painful insults. Sedatives may blur the behavioural pain response. Observational pain assessment tools assess modulated responses from the cortico-cortical circuits and will be influenced from factors as illness of the infants, and inter-variability between the observers, different from pain assessment tools which assess responses from the nociceptive spinal reflex level. The physiological pain assessment tools may be more accurate to assess the size of the nociceptive stimulus.

Conclusions/Results

The physiological pain assessment tools may be most accurate to assess the size of the nociceptive stimulus.

\section{6}

Neonatology

Early introduction of maternal expressed breast milk in the preterm and low birthweight neonatal population Desha Wasala ${ }^{1,2}$, Jyoti Kapur ${ }^{1,2}$, Mabel Molina Garay ${ }^{1,2}$

${ }^{T}$ Neonatal, Royal Stoke University Hospital, UK 


\section{Background}

In 2011 East of England Perinatal Network developed a care bundle which looked at 4 key elements that could lead to prevention of NEC in neonates. We aimed to look at one of these elements and compared it with the practise in a level 3 neonatal unit.

\section{Objective}

To observe the practise of facilitating early introduction of maternal expressed breast milk (EBM) in preterm/low birth weight babies, by commencing early expression (hand and pump expression).

\section{Method}

A retrospective review of babies, 34 weeks and/or $1.8 \mathrm{~kg}$ in birth weight over a 6 month period. We looked at patient notes and the national neonatal database (Badger system) to obtain information about when maternal EBM was initiated and when hand expression, pump expression was first introduced to mothers of preterm/low birthweight babies.

\section{Results}

A total of 54 patients met our gestation and weight criteria; of which 10 mothers had no intension to express breast milk. 43 (98 \%) babies received maternal EBM as their first feed. Only $23(53 \%)$ received EBM within the first 48 hours of life. Fortytwo of the mother's received advice and were shown how to hand express. Forty-one mothers were shown breast pump expression. 2 Babies of this group were treated for confirmed NEC and 2 were treated for suspected NEC.

\section{Conclusion and suggested changes in practice}

We concluded that even though our service is good at using EBM as the first feed, there is still plenty of room for improvement in early introduction of EBM. We plan to implement changes in our trust by introducing breast feeding champions and by producing a new expression log to monitor initiation and on-going support for breast milk expression. We have also introduced the concept of antenatal expression within our unit. We have raised awareness among doctors, neonatal nursing and midwifery teams by presenting our data.

818

\section{Neurology}

Speech Motor Control in Autistic Spectrum Disorders Maureen Casper ${ }^{1}$

${ }^{T}$ Speech Therapy, Rye Child and Adolescent Center for Speech, Language \& Literacy, USA

A fundamental characteristic of autism spectrum disorders ASD is a neurophysiological sensory motor disorder that has a profound impact on the perception and production of spoken language. The sounds of speech are distinctly human and involve neurologically controlled, rapid, coordinated movements of the articulators, shaping of the oral cavity, and vibrations of the vocal folds.

A therapeutic method for developing the production and perception of spoken language in subjects aged 3-10 years was administered. Systematic and controlled auditory and visual stimuli were presented to incrementally increase the range and complexity of the utterances that the child is capable of producing and perceiving.

Case studies presented describe the effects of Speech Motor Training (SMT) and Expressive Language Training (ELT) on speech motor control and language development.

609

\section{Neurology}

Insulin-like growth factor binding protein-3 affects behavior activity through monoaminergic function and synaptic formation Masayuki Itoh ${ }^{l}$, Hongmei Dai ${ }^{l}$

${ }^{T}$ Mental Retardation and Birth Defect Research, National Center of Neurology and Psychiatry, Japan

\section{Background}

Insulin-like growth factor binding protein-3 (IGFBP3) regulates IGF bioactivity, induces apoptosis and inhibits cell growth independent of IGFs, but the functional role of IGFBP3 in the brain is unknown.

Objective

We clarified the effect of IGFBP3 on the brain by characterizing the phenotype of igfbp3-null mice.

\section{Methods and Results}

We established Igfbp3-null mice and used no mutated littermates as wildtype control mice. Compared to wild-type mice, Igfbp3-null mice showed significantly decreased IGF-1 content in the brain but showed no change in weights of brain and body. In igfbp3-null mice the number of dendritic spines was significantly reduced, and the dendritic diameter was thin. In addition, in igfbp3-null mice, a decrease in phosphorylated Akt and ERK1/2 significantly reduced PSD-95 expression, and GAD65/67 expression was significantly decreased. These results indicate that IGFBP3 deficiency impairs neuronal structure and signaling. In behavioral studies, igfbp3-null mice were hyperactive, and a Y-maze alternation test revealed impaired spatial working memory, but no anxiety-like behavior. Monoaminergic analysis using HPLC indicated that igfbp3-null mice showed lower levels of dopamine and serotonin compared to wild-type mice, suggesting an abnormal monoaminergic neurotransmission.

\section{Conclusion}

The studies demonstrated that the deletion of IGFBP3 results in behavioral impairments that are associated with abnormal synaptic function and monoaminergic neurotransmission, which helps to characterize the critical role of IGFBP3 in the brain.

\section{6}

\section{Neurology}

Demand analysis of paediatric high dependency unit (PHDU) for acute neurological morbidity: experience of an Irish regional unit over 7 years

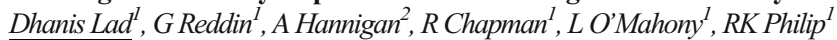
${ }^{T}$ Department of Paediatrics, University Hospital Limerick, Ireland ${ }^{2}$ Department of Statistics, Graduate Entry Medical School, University of Limerick, Ireland

\section{Background}

Paediatric high dependency units (PHDU) care for children who are not unwell enough to be admitted to the Intensive Care Unit (ICU) but require close monitoring and treatments which cannot be provided safely in the general paediatric ward. Acute neurological deterioration among infants and children often require the support from PHDU/ICU, however there is paucity of literature on the true demand for paediatric critical care needs during acute neurological presentations among infants and children. Our study aim is to develop a demand modeling for acute neurological morbidities based on an annual regional birth rate and a childhood population (16 years) for the Mid-West of Ireland.

\section{Method}

Prospectively collected PHDU admission data covering a seven year period was analysed including demographics, diagnosis, management received, length of stay (LOS) and transfer outcomes. Data was verified with computerized hospital inpatient enquiry (HIPE) and analysed using SPSS version 21. Results

Over the study period $(27 \%)$ of primary diagnosis was of the acute neurological category. Convulsions contributed to the highest occupancy, followed by encephalopathy and least common were surgical/head-injury patients. Fifty-three percent were male and $46 \%$ female, with a significant contribution by infants and overall majority being under 10 years of age. Median LOS was 2.6 days. In addition to anticonvulsants $(10.3 \%)$ needed non-invasive respiratory support and $(5.1 \%)$ required endotracheal intubation and ventilation. Two mortalities occurred, $9 \%$ required transfer to ICU/PICU. We estimated a mean annual admission demand of 33 to PHDU for acute neurological presentations requiring 86 bed days per 100,000 childhood population. 


\section{Conclusion}

Our study provides supportive evidence for rationalization in developing critical care facilities available to assist acute care of paediatric neurological patients. Additionally, our observations highlight the PHDU demand of clinical staff to manage those during acute deterioration at regional centres, thus supporting their safe care locally with obvious health economic benefits.

\section{5}

\section{Neurology}

Neurocognitive impairment in children with congenital heart disease

Giulia Sommariva, $^{1,2}$, Anna Jolanda Gortan ${ }^{2}$, Ilaria Liguoro ${ }^{2}$, Martina $\overline{\text { Venchiarutti }^{1,2} \text {, Tiziana Zilli }}{ }^{3}$, Franco Fabbro ${ }^{2}$, Paola Cogo ${ }^{2}$

${ }^{1}$ Department of Neurological Sciences, University of Verona, Italy

${ }^{2}$ Pediatric Clinic, Department of Maternal and Child Health, ASUIUD S.

Maria della Misericordia, University of Udine, Italy

${ }^{3}$ Scientific Institute Eugenio Medea, Scientific Institute Eugenio Medea, San Vito al Tagliamento (Pordenone), Italy

\section{Background}

Congenital heart diseases (CHD) represent the most common type of congenital abnormalities. Thanks to recent improvements in medical and surgical treatment, more than $90 \%$ of children with critical heart defects achieve the adult age. Conversely, survirvors are at risk for neurodevelopmental morbidity.

\section{Objective}

The aim of this study was to evaluate the presence of cognitive dysfunction, behavioural problems and difficulties in social interaction in children with $\mathrm{CHD}$ who underwent heart surgery in cardiopulmonary bypass (CBP).

\section{Methods}

Children aged 6-13 years with a diagnosis of CHD and a previous CBP surgery were included. We used the Wechsler Intelligence Scale for Children - Fourth edition (WISC IV) to evaluate general intelligence, the Conner's Parent Rating Scale - Revised (CPRS-R) and the Child Behaviour Checklist $(C B C L)$ to identify problem behavior in children, and the Adaptive Behaviour Assessment System II (ABAS II) to evaluate adaptive skills.

Results

A total of 17 children ( 7 males, median age $10 \pm 2.18$ ) were enrolled. IQ scores were consistently lower in the studied group than in the standard population $(90.9 \pm 18.3$ vs $100 \pm 15 ; p=.03)$. Perceptual reasoning index $(99.4 \pm 18.3)$ resulted to be higher than verbal comprehension $(90.1 \pm 15.7 ; p=.02)$, working memory $(89.9 \pm 17.7 ; p=.01)$ and processing speed $(90.2 \pm 18.7 ; p=.03)$. The ADHD index evaluated by the CPRS-R was significantly higher in our patients than in the standard population $(64.2 \pm 4.2$ vs $50 \pm 10 ; p=.001)$. Twelve families out of 17 (70\%) filled the CBCL and 5/12 (42\%) reported their children having behavioural issues. According to the ABAS II a significant impairment in adaptive skills emerged in these children in comparison to the standard population ( $86 \pm 30$ vs $100 \pm 15 ; p=.008)$.

Conclusion

Children with $\mathrm{CHD}$ who underwent $\mathrm{CBP}$ surgery presented significant cognitive impairment, behavioural problems and poor adaptive skills. Screening and evaluation of neurodevelopmental delay, along with regular follow-up, are essential to guide appropriate interventions to optimize their development.

\section{9}

Neurology

An Audit of Parents' Knowledge and Confidence in Managing Autistic Children following CYGNET Training Programme at an Integrated Neurodevelopmental Service in South Eastern England Michael Ogundele ${ }^{2}$, Hani Ayyash ${ }^{1}$, Emma Weisblatt ${ }^{1,3}$, Lorraine Cuff ${ }^{l}$, Lorraine Azmi ${ }^{1}$, Tracey Bean ${ }^{I}$, Venkat Reddy ${ }^{1}$

${ }^{1}$ University of Cambridge Health Teaching Trust, Cambridgeshire and Peterborough NHS Foundation Trust, UK
${ }^{2}$ Community Paediatrics, Glenwood Health Centre, NHS Fife, UK

${ }^{3}$ Department of Psychology, University of Cambridge, UK

\section{Background}

Autism Spectrum Disorders (ASD) are neurodevelopmental social communication disorders, with common problems for families including sleep, anxiety, difficulty with change, sensory processing difficulties and disruptive behaviour.

The CYGNET programme was developed in 2003 by the UK-based charity Barnado as an information group for parents of children diagnosed with ASD. It is the primary post-diagnostic intervention offered in the Autism pathway in the Peterborough Neurodevelopmental Service. Parents are routinely invited to attend the 6 session programme which aims to increase their understanding of ASD.

Aim

To evaluate the effectiveness of the CYGNET course in increasing parents' confidence in dealing with common situations relating to their child's ASD. Method

Data was collected retrospectively using an observational study design, using questionnaires completed by parents attending courses over a 12 month period. Sixty-eight parents rated their confidence in dealing with 15 different common situations on a 6 point scale $(0=$ no confidence to $5=$ completely confident), at the start and end of the course. Paired t-test was used to compare total and item scores before and after the course. Fifteen questionnaires were excluded because of incomplete data leaving 53 participants.

\section{Results}

Self-rated the parents'/carers' confidence in dealing with the 15 situations was significantly greater following the programme completion in 51 $(96 \%)$ of the respondents (Figure 1). The mean increase in total score was 18.75 (95\% CI 16.11-21.40), $\mathrm{t}=-14.23, \mathrm{p}$

\section{Conclusion}

This study suggests that the CYGNET programme is effective in increasing the confidence of parents in dealing with difficulties arising when supporting young people with ASD. It would be useful to also evaluate longer-term effects of the CYGNET programme.

Figure 1 showingimprovement in the parents $\%$ carers ${ }^{\circ}$ confidence after CYGNET sessions (Total Point Differences)

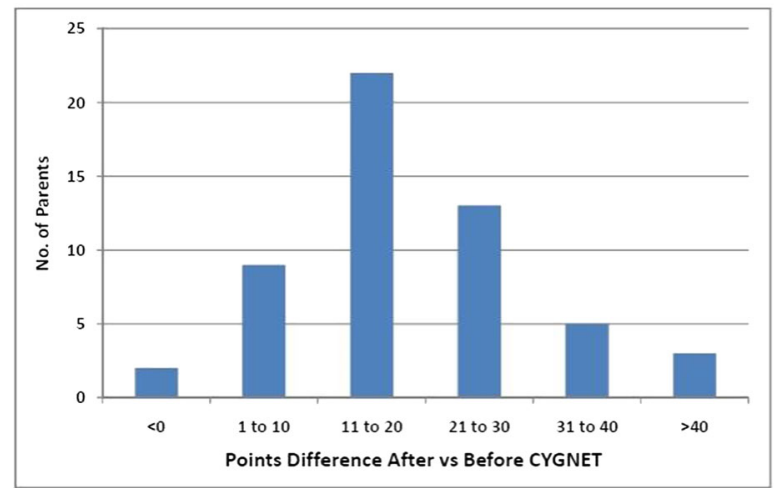

715

Neurology

FDA Labeling Of Antiepileptic Drugs (AEDs) For Partial Onset Seizures (POS) Challenges The European Medicines Agency's (EMA) Pediatric Investigation Plan (PIP) Concept Klaus Rose ${ }^{1}$

${ }^{T}$ Medical Science, klausrose Consulting, Switzerland

\section{Background}

Without a PIP, new drugs cannot be EU-registered. The EMA website lists 7 epilepsy PIPs. In 2016, the FDA decided that AED efficacy in adults can be 
extrapolated to children down to 4 years, making separate safety \& efficacy $(\mathrm{S} \& \mathrm{E})$ trials unnecessary.

\section{Objective}

To investigate whether PIP-demanded pediatric trials are scientifically justified. Methods

All epilepsy PIPs on the EMA website were analyzed. Relevant FDA documents and literature were reviewed.

\section{Results}

The 7 epilepsy PIPs (brivaracetam, EMA-00332-PIP01-08-M11; carisbamate, EMEA-000360-PIP01-08; esclicarbazepine, EMEA-000696-PIP02-10-M05; ethosuximide, EMEA-001617-PIP01-14; lacosamide, EMEA-000402PIP02-11-M03; retigabine, EMEA-000116-PIP01-07-M08; vigabatrin, EMEA-000717-PIP02-13-M02) demand for POS in total 32 studies, 7 modeling \& simulation (M\&S) studies, and one literature review. Patients' ages vary between birth to 17 years, one month to 17 years, 2-17 years, 6-15 years, 1 month to 2 years, 7 to 24 months, 4-17 years, 1 month to 4 years, 5-7 years; 2-11 years, $12-17$ years, \& more. The retigabine studies are to be completed until 2035, the others earlier. The POS studies include open-label S\&E, randomized double-blind placebo-controlled S\&E, M\&S, and other designs. Further PIP indications include refractory pediatric epilepsy syndromes, neonatal seizures, infantile spasms, Lennox-Gastaut Syndrome, childhood absence epilepsy, \& others. The maximum number of PIP modifications was 11 (brivaracetam). The FDA decision is supported by scientific literature, e.g. Dunne et al. 2011, Pellock et al. 2012.

Conclusion

Since the efficacy of AEDs can be extrapolated from adults, most PIPdemanded epilepsy POS trials are medically unjustified and therefore unethical. Children do not need as many clinical trials as possible, only those that truly advance treatment. Clinicians should refuse to participate in such trials or, if already participating in, should reconsider, discuss the FDA's approach with the sponsoring company or clinical research organisation, and ask the responsible ethics committee to consider trial suspension.

307

Neurology

Traumatic Brain Injury in European children - challenges in multidisciplinary care and transition

Mitch Blair ${ }^{1}$, Maria Brenner ${ }^{2}$, Philip Larkin ${ }^{3}$, Austin Warters ${ }^{4}$, Miriam O'Shea ${ }^{5}$

${ }^{T}$ Paediatrics, Imperial College, UK

${ }^{2}$ Nursing, Trinity College Dublin, Ireland

${ }^{3}$ Nursing, University College Dublin, Ireland

${ }^{4} \mathrm{DH}$, Health Services Executive, Ireland

${ }^{5}$ School of Nursing, Trinity College Dublin, Ireland

\section{Background and aims}

Improvements in neonatal and paediatric care mean that more children with complex needs are surviving into adulthood, placing great challenges on healthcare delivery.This paper will present the preliminary findings on the health and social care needs of these children at the community interface, using traumatic brain injury as a tracer condition.

Methods

Date to explore optimum care for these children were gathered across the European Union (EU) and European Economic Area (EEA) as part of a Horizon 2020 funded project Models of Child Health Appraised (MOCHA) running from 2015-18. Country agents compleeted a survey on care processes and interactions between health and social care professionals and parents/carers using a clinical case scenario method.

Results

A number of key issues in the integration of the care for the adolescent with TBI emerged in thsi study to date. These include: limited inclusion of the young person in the planning of their care, limited engagement of family asdvocacy groups, significant absence of policies and procedures to support transition to adult services and limited engagement in data collection to understand the experience of the adolesent and their family.

\section{Conclusion}

The preliminary results suggest that there are limited structures and processes in place to support integrated care delivery to children following a traumatic brain injury across the EU/EEA. Further research is required to explore both regional variations and equity of provision of integrated care for this group of children.

\section{3}

Obesity

Nutritional Behaviour pattern and Physical Activity in obese and overweight Egyptian mothers: Relationships with Their children's body Mass Index Enas Raafat AbdelHamid ${ }^{l}$

${ }^{T}$ Child Health Department, National Research center, Egypt

\section{BACKGROUND}

Obesity and related morbidity increase in Egyptian women and their children. A better understanding of dietary and activity patterns is needed to reduce obesity prevalence.

AIM

The present study aimed to assess dietary patterns and physical activity in Egyptian overweight and obese mothers and to explore its relationships with their children's body mass index (BMI).

\section{SUBJECTS AND METHODS}

This descriptive case-control study was conducted at the National Research Center. The study included a sample of 64 overweight and obese mothers and 75 children, compared with apparently healthy non-obese mothers and their children of matched age and social class. Tested questionnaires were used to collect information of the studied subjects.

\section{RESULTS}

A statistically significantly higher incidence of unemployment, large family size was observed in overweight \& obese women compared to controls ( $\mathrm{P}$ 0.05). Those women who consumed vegetables more than 3 times a week were less likely to be overweight or obese (P 0.05). No significant association were detected between mothers' physical activity, dietary behavior variables and children's BMI except for consuming beverages with added sugar $(95 \% \mathrm{CI}=0.074-0.985, \mathrm{P}$

\section{CONCLUSION}

Improper dietary patterns, nonworking mothers, and big family size are associated with obesity among Egyptian women. Emphasis should be given to increasing physical activity and encourage healthier diets among Egyptian mothers and their children.

626

Obesity

The Malta Childhood National Body Mass Index Study - a population study Samuel Aquilina ${ }^{l}$, Victor Grech ${ }^{1}$, Karl Spiteri ${ }^{2}$, Maria Louisa Busuttil ${ }^{2}$, Erin Camilleri ${ }^{3}$, Victoria Farrugia Sant'Angelo ${ }^{4}$, Neville Calleja ${ }^{5}$

${ }^{1}$ Paediatrics, Mater Dei hospital, Malta

${ }^{2}$ Research unit, Malta Association of Physiotherapists, Malta

${ }^{3}$ EU and International Affairs, Research and Policy Development Directorate, Malta

${ }^{4}$ Child Health Services, Primary Health Care, Malta

${ }^{5}$ Health Information and Research, Primary Health Care, Malta

\section{Introduction}

Obesity is a chronic disease that often commences in childhood. Over quarter of Maltese children are overweight or obese. This study was carried out in order to measure height and weight (and Body Mass Index) for all school children in Malta in order to precisely quantify the extent of the problem.

Methods

Schooling in Malta is provided by: A. Free State schools, B. Subsidised Roman-Catholic Church-run schools and C. Independent Private schools. 
All were included. Physical education teachers were trained in measurements on identical stadiometers. Bespoke spreadsheets were created using World Health Organisation cut-offs for underweight, overweight and obesity.

\section{Results}

This study included over 45889 children in over 145 schools (ages 4.7-17years). Less than $10 \%$ were unmeasured. Circa $40 \%$ of school-aged children in Malta are overweight or obese. The proportion of obese was greater than of overweight. Levels of overweight and obesity were significantly different: StateChurchIndependent schools. Overall, and for both genders and for school types, there was a trend for overweight and obesity to peak in Years $5-8$, then decline slightly. Overweight and obesity was secondaryprimary schools, and malesfemales. The underweight group was small with no significant difference between the school types.

\section{Conclusion}

This study has confirmed high levels of overweight and obesity in Maltese children. It also provides proof of concept of scalability by demonstrating the feasibility of undertaking a relatively inexpensive study of an entire childhood population. The modus operandi (utilising physical education teachers) could relatively easily be up scaled for any country.

\section{0}

\section{Primary Care}

Prevention of Adult Cardiovascular Diseases in Emirati Children - A Preliminary Report

Elhadi Aburawi ${ }^{1}$, Hassib Narchi ${ }^{1}$, Lolowa Al Mukhini ${ }^{1}$, Javed Yasin ${ }^{1}$, Sania Al Hamad $^{1}$, A-K Souid ${ }^{l}$

${ }^{1}$ Pediatric, CMHS, UAE University, United Arab Emirates

\section{Background/Hypothesis}

Atherosclerosis starts early in life and remains asymptomatic in children. Its progression is hastened by obesity, hypertension, diabetes, and dyslipidemia. Early recognition and prompt intervention of these adverse events would halt the development of cardiovascular diseases (CVD) in adults.

\section{Aims}

(1) Determine the prevalence of obesity and hypertension in Emirati children; and (2) Engage students with increased susceptibility to CVD in risk-reduction and counselling programs.

\section{Methods}

Study subjects were children in grade 2 (elementary school), 6 (middle school) and 10 (high school). Parents completed the study questionnaire, which addressed consanguinity and CVD risk factors. Students had height, weight, body-mass-index (BMI), waist circumference and blood pressure (BP) determinations. The International Obesity Task Force (IOTEF) criteria were used for BMI classification.

\section{Results}

Five-hundred-thirty-five students were included in this study; their mean [SD] age was 10.7 [11] years $(\mathrm{M}: \mathrm{F}=1: 1)$. Risk factors identified in their fathers were smoking (21\%), hypertension (14\%), dyslipidemia (12\%), heart attacks (3\%), diabetes $(16 \%)$, and obesity (9\%). Risk factors identified in their mothers were hypertension $(4 \%)$, dyslipidemia $(5 \%)$, diabetes $(6 \%)$, and obesity (3\%). The prevalence of consanguinity was $38 \%$. For male students, the prevalence of thinness was $31 \%$, overweight $14 \%$, obesity $6 \%$, and extremely-obesity $3 \%$. For female students, the prevalence of thinness was $40 \%$, overweight $9 \%$, obesity $5 \%$, and extremely-obesity $3 \%$ (Fig. 1). The z-scores of systolic and diastolic BP increased with increased excess body fat (Fig. 2). Fig. 3 represents the waist circumference percentiles in function of age.
Fig. 1. IOTEF classification of the BMI of the studied students

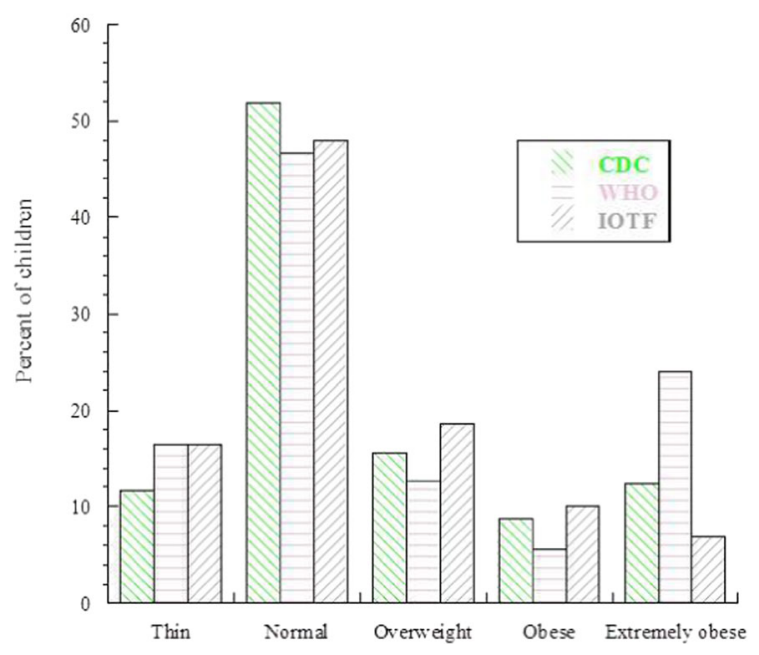

Fig. 2. Systolic and Diastolic BP z-scores in relation to excess body fat.
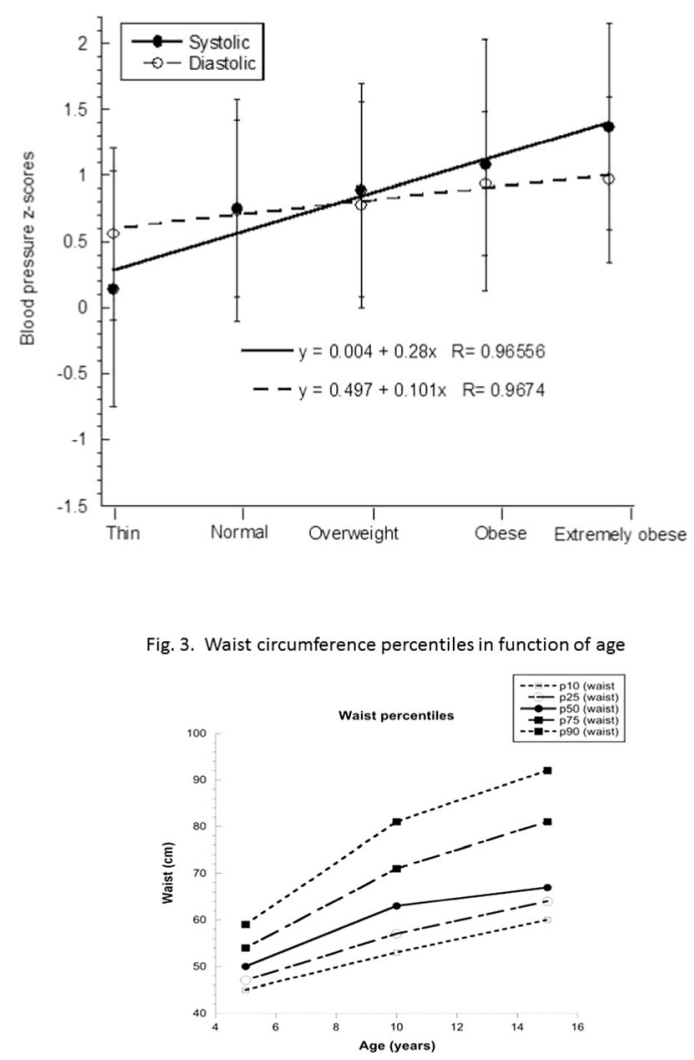

643

Primary Care

The experiences, attitudes and perceptions of paediatricians in Malta relating to child protection work: implications of a mixed-methods study Kevin Borg ${ }^{1,3}$, Mariella Mangion $^{1}$, Jane Barlow ${ }^{2}$

${ }^{7}$ Child and adolescent Health, Mater Dei Hospital, Malta

${ }^{2}$ Social Policy and Intervention, University of Oxford, UK

${ }^{3}$ Warwick Medical School, University of Warwick, UK 
Health professionals fulfil an essential role within the multiagency response to safeguarding concerns. This study aimed to explore the behaviours and perceptions of paediatricians relating to Child Protection (CP) work in a country characterised by the absence of mandatory and statutory guidance. A mixedmethods approach was adopted, using the explanatory sequential design. The quantitative strand involved a population survey of paediatricians $(N=56)$. Data were analysed and used to inform a subsequent qualitative phase, in which data were collected using one-to-one interviews and analyzed using thematic analysis. The response rate was at $95 \%(n=53)$. Paediatricians perceived themselves to be working within an unstructured system. Despite the majority reporting to have assessed CP cases, $77.4 \%(n=41)$ had never received specific $\mathrm{CP}$ training. Furthermore, the majority reported that response pathways and reporting procedures were unclear and that they were not involved in CP related tasks beyond identification. Both quantitative and qualitative data indicated that $\mathrm{CP}$ work was perceived as complex, emotive and stressful, and that this, together with lack of experience, resulted in ambivalence and lack of confidence particularly in child sexual abuse. Although participants felt reassured by having a designated consultant, this role also enabled them to offload responsibility, with CP work not being fully owned. Trainees appeared more willing to be involved than specialists. This study suggests that the lack of mandatory and statutory guidance results in significantly suboptimal practice with regard to CP. Investment in formalised structured training and support systems that enhance reflective practices, are recommended to engage professionals in CP work. Designated professionals in $\mathrm{CP}$ should complement, rather than minimise, the role and responsibility of professionals working with children. Such measures will help create a cognitivie change to making $\mathrm{CP}$ everybody's responsibility and that ensure a more robust and sustainable $\mathrm{CP}$ system that is child-centred and more costeffective.

\section{4}

\section{Primary Care}

Reducing Low-value Care; Top 5 Priorities for Australasian Paediatricians

Sarah Dalton $^{1,2}$, Jason Soon ${ }^{1}$, Harriet Hiscock ${ }^{1,3,4}$

${ }^{T}$ Paediatrics and Child Health Division, The Royal Australasian College of Physicians, Australia

${ }^{2}$ Emergency Department, The Children's Hospital at Westmead, Australia

${ }^{3}$ Health Services Research Unit, The Royal Children's Hospital, Australia

${ }^{4}$ Department of Paediatrics, The University of Melbourne, Australia

\section{Background}

Evolve is an initiative of the Royal Australasian College of Physicians (RACP) to identify and reduce low-value medical practices. RACP specialties participate by producing a list of their 'top five' low value practices to lay the ground for clinical change.

Objectives

In 2016, the RACP's Paediatrics and Child Health Division set out to produce a top five list of low value practices for general paediatrics.

Methods

Initially a list of all paediatric-related clinical practices already identified as 'low value' by other specialties in Australasia and overseas was compiled. A rapid review of evidence was performed to confirm that practices were of low value, and 15 shortlisted for further consideration. Evidence summaries for each were reviewed at a workshop and participants voted on top 10 practices. Following this an email survey asked respondents to indicate for each of the 10 practices, whether: (i) the practice was commonly undertaken in paediatric patients, (ii) there was good evidence to undertake the practice less often, and (iii) reducing its use was important in reducing harm and/or costs to the healthcare system.

Results

Survey response rate was $22 \%(n=269)$. Table 1 shows the ranking from highest to lowest of practices for all respondents, 'public sector' and 'private sector' respondents. Ranking was similar across practice groupings except for frenotomy.

\section{Conclusion}

We believe the general paediatric Evolve list provides good clinician agreement as to low value practices. The challenge now is to measure in a valid and reliable way where these practices are occurring and then develop, test and translate sustainable interventions to reduce them.

\begin{tabular}{|c|c|c|c|}
\hline Rank & $\begin{array}{l}\text { Al } \\
\text { Do not routinely.... }\end{array}$ & $\begin{array}{l}\text { Public sector } \\
\text { Do not routinely.... }\end{array}$ & $\begin{array}{l}\text { Private sector } \\
\text { Do not routinely.... }\end{array}$ \\
\hline 1 & $\begin{array}{l}\text { prescribe oral antibiotics to } \\
\text { children with fever without } \\
\text { an identified bacterial } \\
\text { infection }\end{array}$ & $\begin{array}{l}\text { prescribe oral antibiotics to } \\
\text { children with fever without } \\
\text { an identified bacterial } \\
\text { infection }\end{array}$ & $\begin{array}{l}\text { advise frenotomy for the } \\
\text { relief of ankyloglossia in } \\
\text { newborns }\end{array}$ \\
\hline 2 & $\begin{array}{l}\text { undertake chest X-rays for } \\
\text { the diagnosis of } \\
\text { bronchiolitis in children or } \\
\text { routinely prescribe } \\
\text { salbutamol or systemic } \\
\text { corticosteroids to treat } \\
\text { bronchiolitis in children }\end{array}$ & $\begin{array}{l}\text { treat gastroesophageal } \\
\text { reflux disease in infants } \\
\text { with acid suppression } \\
\text { therapy }\end{array}$ & $\begin{array}{l}\text { prescribe oral antibiotics to } \\
\text { children with fever without } \\
\text { an identified bacterial } \\
\text { infection }\end{array}$ \\
\hline 3 & $\begin{array}{l}\text { treat gastroesophageal } \\
\text { reflux disease in infants } \\
\text { with acid suppression } \\
\text { therapy. }\end{array}$ & $\begin{array}{l}\text { undertake chest X-rays for } \\
\text { the diagnosis of } \\
\text { bronchiolitis in children or } \\
\text { routinely prescribe } \\
\text { sabutamol or systemic } \\
\text { corticosteroids to treat } \\
\text { bronchiolitis in children }\end{array}$ & $\begin{array}{l}\text { undertake chest X-rays for } \\
\text { the diagnosis of } \\
\text { bronchiolitis in children or } \\
\text { routinely prescribe } \\
\text { salbutamol or systemic } \\
\text { corticosteroids to treat } \\
\text { bronchiolitis in children }\end{array}$ \\
\hline 4 & $\begin{array}{l}\text { order chest X-rays for the } \\
\text { diagnosis of asthma in } \\
\text { children }\end{array}$ & $\begin{array}{l}\text { order abdominal X-rays for } \\
\text { the diagnosis of non- } \\
\text { specific abdominal pain in } \\
\text { children }\end{array}$ & $\begin{array}{l}\text { order chest X-rays for the } \\
\text { diagnosis of asthma in } \\
\text { children }\end{array}$ \\
\hline 5 & $\begin{array}{l}\text { order abdominal X-rays for } \\
\text { the diagnosis of non- } \\
\text { specific abdominal pain in } \\
\text { children }\end{array}$ & $\begin{array}{l}\text { order chest X-rays for the } \\
\text { diagnosis of asthma in } \\
\text { children }\end{array}$ & $\begin{array}{l}\text { order abdominal X-rays for } \\
\text { the diagnosis of non- } \\
\text { specific abdominal pain in } \\
\text { children }\end{array}$ \\
\hline 6 & $\begin{array}{l}\text { advise frenotomy for the } \\
\text { relief of ankyloglossia in } \\
\text { newborns }\end{array}$ & $\begin{array}{l}\text { advise frenotomy for the } \\
\text { relief of ankyloglossia in } \\
\text { newborns }\end{array}$ & $\begin{array}{l}\text { order baseline blood tests } \\
\text { just because an } \\
\text { intravenous cannula has } \\
\text { been placed in a paediatric } \\
\text { patient }\end{array}$ \\
\hline 7 & $\begin{array}{l}\text { order baseline blood tests } \\
\text { just because an } \\
\text { intraverous cannula has } \\
\text { been placed in a paediatric } \\
\text { patient }\end{array}$ & $\begin{array}{l}\text { order baseline blood tests } \\
\text { just because an } \\
\text { intravenous cannula has } \\
\text { been placed in a paediatric } \\
\text { patient }\end{array}$ & $\begin{array}{l}\text { treat gastroesophageal } \\
\text { reflux disease in infants } \\
\text { with acid suppression } \\
\text { therapy }\end{array}$ \\
\hline 8 & $\begin{array}{l}\text { undertake allergy testing } \\
\text { such as skin prick tests or } \\
\text { blood tests for the routine } \\
\text { evaluation of eczema }\end{array}$ & $\begin{array}{l}\text { undertake allergy testing } \\
\text { such as skin prick tests or } \\
\text { blood tests for the routine } \\
\text { evaluation of eczema }\end{array}$ & $\begin{array}{l}\text { undertake allergy testing } \\
\text { such as skin prick tests or } \\
\text { blood tests for the routine } \\
\text { evaluation of eczema }\end{array}$ \\
\hline 9 & $\begin{array}{l}\text { request an EEG after a } \\
\text { child's frst unexplained } \\
\text { afebrile seizure }\end{array}$ & $\begin{array}{l}\text { request an EEG after a } \\
\text { child's first unexplained } \\
\text { afebrile seizure }\end{array}$ & $\begin{array}{l}\text { use selective serotonin } \\
\text { reuptake inhibitors as the } \\
\text { first-line intervention for } \\
\text { mild to moderately } \\
\text { depressed children and } \\
\text { young people }\end{array}$ \\
\hline 10 & $\begin{array}{l}\text { use selective serotonin } \\
\text { reuptake inhibitors as the } \\
\text { first-line intervention for } \\
\text { mild to moderately } \\
\text { depressed children and } \\
\text { young people }\end{array}$ & $\begin{array}{l}\text { use selective serotonin } \\
\text { reuptake inhibitors as the } \\
\text { first-line intervention for } \\
\text { mild to moderately } \\
\text { depressed children and } \\
\text { young people }\end{array}$ & $\begin{array}{l}\text { request an EEG after a } \\
\text { child's first unexplained } \\
\text { afebrile seizure }\end{array}$ \\
\hline
\end{tabular}

355

\section{Primary Care}

Development of a Core Set of Quality-Indicators for Paediatric Primary Care Practices in Europe, COSI-PPC-EU

Dominik Ewald ${ }^{1}$, Gottfried Huss ${ }^{2}$, Silke Auras ${ }^{3}$, Juan Ruiz-Canela Cáceres $^{4}$, Max Geraedts ${ }^{5}$

${ }^{1}$ Drs. Heuschmann, Ewald, Paediatric Primary Care Centre, Germany

${ }^{2}$ President, European Association of Primary Care Paediatricians, ECPCP, Germany

${ }^{3}$ Institute for Health System Research, Faculty of Health, Witten/ Herdecke University, Germany

${ }^{4}$ Pediatra, Centro de Salud Virgen de África, Spain

${ }^{5}$ Institute for Health Services Research and Clinical Epidemiology, Faculty of Medicine, University of Marburg, Germany

Paediatric Primary Care is different in each European country and its health care system. Striking quality differences have been shown in the delivery and outcomes of child health care. Thus there is a need to measure and improve quality in Paediatric Primary Care in Europe.

The aim of this study was to develop a set of Quality Indicators (QI) to assess and monitor Paediatric Primary Care (PPC) in Europe. In a three- 
step process we used the available evidence from literature and European expert consensus in a modified RAND/UCLA Appropriateness Method to develop the indicator set. A broad literature and online research of published QI and guidelines yielded a huge inventory of 1516 QI. A collaborative panel of paediatric senior experts from the European Academy of Paediatrics (EAP) and the European Confederation of Primary Care Paediatricians (ECPCP) from 15 European countries participated in two consensus processes. They reduced the initial indicator inventory by rating the QI regarding validity and feasibility. After the second consensus process the QI set "COSI-PPC-EU" consists of 42 indicators in five categories of paediatric primary care: (A) health promotion/prevention/screening (13 QI), (B) acute care (9 QI), (C) chronic care (8 QI), (D) practice management (3 QI) and (E) patient safety (9 QI).

\section{Conclusion}

A consented set of a limited number of valid quality indicators is presented for the application in paediatric primary care in different health care systems throughout Europe.

\section{8}

\section{Primary Care}

The Implementation situation of health management for children aged 0-6 years: a typical study on the national basic public health services Ruili Li ${ }^{1}{ }^{*}$, Xiaoguo Zheng, Xin Liu, Yalan Zhang, Lihong Wang, Bowen Chen ${ }^{T}$ Children Health and Development Department, Capital Institute of Paediatrics, China

\section{Background}

The national basic public health service is the main content of the new medical reform and the important component of the public health system construction in china. Health management of children aged 0-6 is one of the important content, and is provided by the government free of charge, but parents compliance is not very high, this study aims to Investigate the present situation and the reasons in order to improve the implementation of the government project.

\section{Methods}

A typical survey in a community health service center. We collected and analyzed the electronic health records data of the children aged 0-6 to understand the utilization situation of the children's health management provided by the community health service institution.

\section{Results}

A total of 5344 children's health records were investigated, of which male was 2707 and female was 2637, accounting for $50.7 \%$ and $49.3 \%$, respectively. The percentage of hemoglobin in children aged $0-3$ years, 4-6 years of hemoglobin and the proportion of children who had a hearing screening were $75.56 \%, 46.17 \%$ and $65.96 \%$, respectively. During the follow-up period, the prevalence of the disease was filled out "no abnormal cases". But there were 680 cases of abnormal conditions, and only two of the referral recommendations was given which were not required by the service specification. The content of health education guidance is stereotyped, mainly for the general content. Overweight and obese children accounted for $20 \%$, but did not give relevant guidance. Conclusions

In order to improve the level of children's health, it is necessary to increase the utilization rate of the government's free service through developing the knowledge and skills of medical staff in the community health service and the parents' awareness of routine health care for children.

\section{2}

\section{Primary Care}

Fever Phobia: Parental Perceptions and Management of Pyrexia Karen McCarthy ${ }^{l}$, Ike Okafor, Roisin McNamara

${ }^{T}$ Paediatric Emergency Medicine, Temple Street Children's University Hospital, Ireland

\section{Introduction}

The febrile child is a common presentation. The aim of the study was to determine parental definition and management of pyrexia and to identify areas where parental education would be of benefit.

\section{Methods}

A qualitative questionnaire based study was undertaken. A single interviewer administered 100 questionnaires over a 5-day period to parents attending the Emergency Department. Verbal consent was obtained from all parents/guardians prior to completing the questionnaire.

\section{Results}

One hundred parents/guardians participated in the study. Seventy-five percent were mothers with a mean age of 35.8 . The majority were Irish and $88 \%$ had access to a thermometer at home. Parental definition of fever ranged from 36.5 to $40^{\circ} \mathrm{C}$. Sixty percent of parents define fever at a different limit than defined in ICD-10 $\left(38{ }^{\circ} \mathrm{C}\right)$. Fifty-four percent of parents/guardians reported that they worry more about fever in infants oC. Reported dosing schedules for Paracetamol were inappropriate in $62 \%$ and for Ibuprofen in $57 \%$. Twenty-one percent of parents feel that all children with fever should be treated with antibiotics and $22 \%$ immediately seek medical attention if they perceive their child has a fever. Sixty percent of parents would consult their GP initially if their child had a fever. Sixty-four percent of parents would describe themselves as fever phobic and $60 \%$ are not confident in their ability to manage their child's temperature at home. Twenty-nine percent of patients had previously received education about fever in children although $99 \%$ of those interviewed felt education would be of benefit.

\section{Discussion}

Fever phobia is a common phenomenon among parents and guardians of children attending the emergency department with $64 \%$ of parents describing themselves as fever phobic. Furthermore parents lack confidence in managing fever at home. There are many parental misconceptions about fever and its management. Education regarding the management of fever is lacking and $99 \%$ of parents would welcome formal education on this subject.

\section{5}

\section{Primary Care}

Performing spinal ultrasounds on babies with simple sacral dimples in a regional neonatal centre Carmel Maria Moore ${ }^{1}$, Jonathan Soye ${ }^{2}$, John Fitzsimons ${ }^{1}$ ${ }^{T}$ Department of Paediatrics, Our Lady of Lourdes Hospital, Ireland ${ }^{2}$ Department of Radiology, Our Lady of Lourdes Hospital, Ireland

Anecdotally in our centre parents reported anxiety on waiting for results of spinal ultrasounds, so this study's objective was to investigate the number of spinal ultrasounds performed and the indications for performing them on babies under six months of age in our regional neonatal centre. It also aimed to investigate the frequency of abnormal ultrasound results.

\section{Methods}

The radiology imaging management system was used to provide cases for a retrospective review of cases of spinal ultrasound performed on infants aged less than six months in our centre. All data recorded was anonymised.

\section{Results}

Just over $1 \%$ of babies $(n=32)$ who were born in the preceding calendar year underwent a spinal ultrasound. Infants ranged in age from 3 days to 6 months. A sacral dimple was the most common reason for requesting a spinal ultrasound with $65.6 \%(n=21)$ performed for this reason. Over $12 \%(n=4)$ of scans were performed for spinal haemangiomas. Just over $81 \%(n=26)$ of scans detected no anomaly. Three $(9.4 \%)$ scans were abnormal, with all three having cord tethering: none of these infants had only a sacral dimple or a tuft of hair. All three babies were referred for neurosurgical review and as of yet no baby has required intervention.

\section{Conclusions}

The most common reason for performing spinal ultrasounds in our regional paediatric and neonatal centre is still sacral dimples and all ultrasounds 
performed for this reason were normal. We plan to create a local guideline to prevent unnecessary imaging of babies with simple sacral dimples.

293

\section{Primary Care}

The Impact of Innovative medical Information Integration System on clinical research in Japan

Yoshihiko Morikawa $^{1}$, Shogo Kato ${ }^{2}$, Naohisa Yahagi ${ }^{3}$

${ }^{T}$ Clinical Research Support Center, Tokyo Metropolitan Children's Medical Center, Japan

${ }^{2}$ Division of Data Science for Clinical Research, National Center for Child Health and Development, Japan

${ }^{3}$ Guraduate School of Media and Governance, Keio University, Japan

\section{Background}

Advances in information and communication technology supports many aspects of medical practices and clinical researches. Data collection using case report form $(\mathrm{CRF})$ requires manual works and makes burden to researchers and cost. A system that assembles and manages data from different formats and multiple data sources into a centralized system, Clinical data management system (CDMS), has been introduced in Japan since 2014.

Objective

To describe the function of innovative database, CDMS, and feasibility of implementing and using sharable and comparable medical data for clinical research.

Design/Methods

CDMS, a user-friendly, versatile, and secure network system for data and knowledge management has been assembled in Japan. CDMS for patients was introduced to outpatient department to take patient history and interview. CDMS integrates disease, laboratory data, and prescription from multiple platforms of electronic health record in each institute and patients' medical interview and history.

To evaluate the feasibility of CDMS for clinical research, the observational items used in CRFs of clinical trial conducted at Tokyo Metropolitan Children's Medical Center in 2016 were sorted and the proportion of items that could theoretically be covered by CDMS was evaluated.

Results

The versatile database covered everything about pediatrics from infants to child medical records. CDMS used a metadata-driven model that contained built-in knowledge bases to control medical terminology. Among the CRFs from 4 clinical trials, patient medical interview and history, disease, prescription and laboratory data accounted for $33.0 \pm 39.4 \%, 0.6 \pm 0.9 \%$, $18.6 \pm 16.7 \%$ and $21.8 \pm 12.3 \%$, respectively. CDMS covered $73.9 \pm 41.4 \%$ of observational items in clinical research.

Conclusions

CDMS broadly covered the observational items in clinical research and was showed the usefulness for it. CDMS also functions as a platform to realize randomized registry trial and will reveal the real-world evidence in clinical care, home or community settings. CDMS will change clinical research from hypothesis testing to confirming the correlation between the big data and every medical thing.

730

\section{Primary Care}

Neurodevelopmental-Related Health Inequalities among Children Screened for Biotinidase Deficiency (BTD) In a Local Scottish Local Authority Michael Ogundele ${ }^{1}$, Abdulbaset Noaman ${ }^{1}$, Heather Holmes ${ }^{2}$, Mary Jarvie $^{2}$

${ }^{1}$ Community Paediatric Unit, NHS Fife, UK

${ }^{2}$ Clinical Biochemistry Department, NHS Fife, UK

\section{Background}

Deprivation is the key determinant of health inequalities, accounting for the unequal socio-economic patterning of outcomes which disadvantages less affluent children. It is influenced by a wide range of factors including access to education, employment and good housing, access to healthcare, income levels and genetic factors.

Aims

To examine socioeconomic inequalities (SEI) in the incidence of clinical diagnoses among children screened for BTD in Local Authority (LA).

Methods

We retrospectively reviewed the clinical and laboratory data of all children throughout NHS Fife area screened for Biotinidase deficiency (BTD) over a two- year period between July 2014 and July 2016.

SEI was calculated using the Slope Index of Inequality (SII) and the Relative Index of Inequality (RII) based on the linear regression coefficient between the proportion of patients in each $20 \%$ (Quintile) of data zones for each patient and the hierarchical ranking value of each Quintile. The RII is calculated by dividing SII with the mean level of patient proportion.

\section{Results}

Analysis of the patients' Neurodevelopmental diagnosis confirmed that only seven out of 40 different diagnostic conditions showed evidence of significant socio-economic inequalities in their distribution among children and youth screened for BTD, i.e. Global Developmental Delay (GDD), Speech and Language delay (SALD), Attention deficit and Hyperactive Disorder (ADHD), Social Communication difficulties (SCD) but not Autistic Spectrum Disorder, Sleep and Behaviour difficulties, and Genetic disorders (Table 1). RII was 0.9 to 1.3 for the seven conditions.

Conclusion

This study has shown that children in the most deprived areas of the LA have significantly worse health compared to those living in other areas with regards to some neurodevelopmental conditions mainly Genetic disorders, Global, Speech, Language and Social Communication delays, ADHD, behavioural and sleep difficulties. Similar studies in other Scottish LA will be helpful to compare this finding among the children population.

Table 1 showing the Neurodevelopmental disorders affected by significant socioeconomic inequalities
\begin{tabular}{|l|c|c|c|c|c|c|c|c|}
\hline Diagnosis & No_Pts & Percent & Pop_Prev & Q1_Ptp & Q5_Ptp & Mean & SII & RII \\
\hline GDD & 85 & 35 & 1.33 & 1.9 & 0.7 & 1.3 & -1.65 & -1.3 \\
\hline SCD & 60 & 24.7 & 0.94 & 1.2 & 0.5 & 0.92 & -0.95 & -1 \\
\hline Sleep & 52 & 21.4 & 0.81 & 1.3 & 0.2 & 0.78 & -1.55 & -2 \\
\hline Behaviour & 46 & 18.9 & 0.72 & 0.9 & 0.2 & 0.72 & -0.85 & -1.2 \\
\hline SALD & 34 & 14 & 0.53 & 0.7 & 0.3 & 0.5 & -0.5 & -1 \\
\hline ADHD & 33 & 13.6 & 0.51 & 0.7 & 0.3 & 0.5 & -0.45 & -0.9 \\
\hline Genetic & 6 & 2.5 & 0.09 & 0.2 & 0 & 0.1 & -0.2 & -2 \\
\hline
\end{tabular}

Legend: ${ }^{S}$ Population prevalence per $1000 ;{ }^{\beta}$ Quintile 1 (Most deprived) Patient prevalence ${ }^{*}$ Quintile 5 (Least deprived) Patient prevalence: GDD - Global Developmental Delay: SALD - Speech and Language delay; ADHD - Attention deficit and Hyperactive Disorder; SCD - Social Communication Difficulties

\section{6}

\section{Primary Care}

Female Genital Mutilation among Egyptian School Children Iman Salama ${ }^{1}$ Samia Sami ${ }^{3}, \underline{\text { Thanaa Rabah }}{ }^{2}$, Somaia Salama ${ }^{1}$, Aida Abdel Mohsin ${ }^{1}$

${ }^{1}$ Community Medicine Research Department, Professor Researcher Dr., Egypt ${ }^{2}$ Community Medicine Research Department, Assistant Professor Researcher Dr., Egypt

${ }^{3}$ Child Health Department, Professor Researcher Dr., Egypt

\section{Objective}

To assess the prevalence of female genital mutilation (FGM) among Egyptian school children and associated sociodemographic risk factors.

\section{Methodology}

A national community based survey on a 60 school facility level was carried out on 1008 female children aged from 8 to 18 years from 6 governorates representing Egypt. Written consents from child parents and verbal ascent from children aged 10 years were attained. A face to face interview was carried out to fulfil the designed questionnaire during the period from Mars 2013 to April 2015. 


\section{Results}

High percent of the studied girls $(48.6 \%)$ were mutilated. The percent of girls with FGM was significantly higher among girls aged above 15 years $(60.5 \%)$ compared to those aged $8-12$ and 12 to 15 years $(22 \% \& 49 \%$ respectively) P 0.001 . The highest percent with FGM was found among girls from Assuit and Beni-suef governorates-representing Upper Egypt (70\% and $60 \%$ respectively) compared to Lower Egypt or Cairo the capital ( $42.8 \%$ \& $25.5 \%$ respectively), P 0.001 . The FGM was significantly higher among girls from rural areas $(63 \%)$ compared to $33 \%$ in urban areas, P 0.001 . The FGM was significantly related to father and mother education, as females whose father or mother have secondary or university education were mutilated (35\% and $40 \%$ respectively) compared to $58.4 \%$ and $56.0 \%$ respectively among those with lower education, $\mathrm{P} 0.001$. There was no significant relation between FGM and family income (p0.05). FGM was done mainly by doctors (64\%) especially in urban areas as it reached to about $75 \%$. About $20 \%$ of mutilation was done by "Dayas".

\section{Conclusion}

Although the awareness programs about the health hazards of FGM were carried out, FGM is still a major problem in Egypt especially among girls living in rural areas especially in Upper Egypt and those with lower educated population.

\section{3}

\section{Primary Care}

Development of a Gold Standard Service for Children in Public Care (Looked After Children) in a University Teaching Hospital in the UK Thomas Heron ${ }^{1}$, Rosalind Rabone ${ }^{1}$, Varsha Sadavarte ${ }^{l}$

${ }^{T}$ Community Paediatrics, University Hospital North Midlands, UK

\section{Background}

Looked after children (LAC) are a vulnerable group of society who have signficant healthcare needs. In 2016 there were 70,000 looked after children in England. Each year this number has risen by $1 \%$ and includes growing numbers of unaccompanied asylum seeking children.

\section{Objective}

Following RCPCH \& NICE recommendations, we created a streamlined, standardised healthcare tool for the initial health assessment (IHA) of children in public care. By sharing our experiences, we hope to contribute towards practices in other centres.

Methods

All children placed into care within the region are referred by social services to a specialized LAC health team. Social services reports and parental health-assessments are sent using a secure online system (Part A). Part B allows young persons to consent.

There are five protected consultant-led clinics, creating a minimum of 10 IHA medicals a week. A paediatrician will complete Part C (child/young person/asylum seeker) detailing the implications of social, education and health information for the child's current and future wellbeing. This comprehensive report is a standardised pro-forma and evidence based medicine statements are used for health implications to create consistency amongst clinicians.

A strict 20 day completion ensures multiagency professionals focus on timely care. The completed report is securely shared with general practitioners, social services and other essential agencies.

To enhance practice, all clinicians complete an online health data survey after each consultation. We ask carers for feedback to monitor satisfaction and aid quality improvements.

\section{Conclusion}

Looked after children are a vulnerable group of society and their exposure to adverse life events is associated with poorer outcomes across social, education and health. Tackling these health inequalities requires multiagency collaboration. We have successfully created a quality standard health tool to facilitate this. Sharing information, gaining feedback and working together is pivotal for a holistic service for these children.
459

\section{Primary Care}

Congenital heart disease: a population study in Friuli Venezia Giulia, Italy. Enrico Petoello ${ }^{I}$, Michela Vergine ${ }^{I}$, Francesca Rech Morassutti ${ }^{I}$, Carlo De Pieri ${ }^{l}$, Anna Pusiol ${ }^{1}$, Paola Cogo

${ }^{I}$ Department of Clinical and Experimental Medical Science, University Hospital of Udine, Pediatric Clinic, Italy

\section{Background}

Congenital heart disease (CHD) is the most common and severe class of major congenital malformations. Despite great interest in pathogenesis and surgical outcome of individual CHD, data from population studies on CHDs as a whole, especially in our region, are missing.

Objective

To assess epidemiology and population characteristics of children with CHD in Friuli Venezia Giulia (FVG), Italy

\section{Methods}

Children born in FVG between May 7, 2003 and December 1, 2015 with at least one ICD-9 diagnosis of CHD, and one or more admission to the University Hospital of Udine were enrolled. Hospital discharge data were retrospectively reviewed and the collected data were combined in a database including demographic data, cause of admission, eventual surgery and outcomes.

\section{Results}

Five hundred ninety-four children, of which $48.8 \%$ male and $46.5 \%$ preterm, were included: median age was 69 months; mortality was $8.4 \%$. CHD diagnosis and relative frequency are listed in figure $1.15 .3 \%$ of patients had surgery, the majority of which underwent multiple procedures. Comorbidities were diagnosed in $29.8 \%$ of patients, with surgical patient being more affected $(50.5 \%$ vs $26 \% ; p=0.02)$. Primary cause of hospitalization were congenital malformations in surgical patients $(47.8 \%$ vs $22 \% ; p=0.03$ ), and perinatal problems in non surgical ones (32.9\% vs $6.5 \%$ respectively; $p=0.01$ ). Cardiac disease was cause of hospitalization in $7.4 \%$ of surgical patient and $1.5 \%$ in non surgical ones. Number of admission and length of hospitalization appeared to be grater in surgical patients, although the difference was not statistically significant.

\section{Conclusions}

Our database describes a considerable pathological heterogeneity. Comorbidities seem to play an important role in children with CHD. Cardiac disease is cause of hospital admission less often than expected: this findings stress the necessity to assist the patient with CHD through a multidisciplinary and inclusive approach.

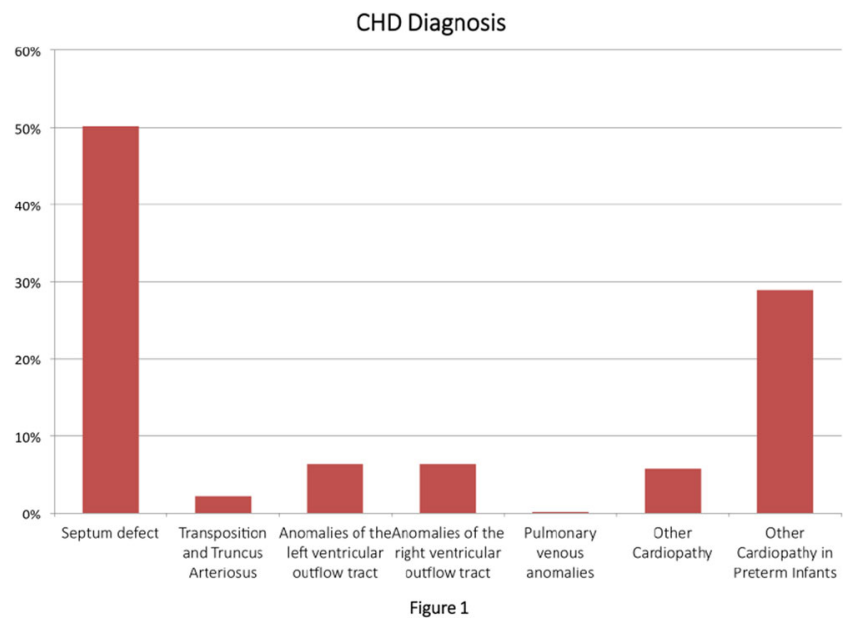


809

\section{Primary Care}

Dr Jargon Paediatrics: A Teaching Resource to Help Healthcare Professionals Improve Paediatric Communication Skills

Victoria Rodulson

${ }^{T}$ Paediatrics, Raigmore Hospital, UK

\section{Background}

Medical students can expect to learn 'upward of 10,000 new words' (Kagan, 2011); it is therefore not surprising that children do not always understand us. Children report feeling confused and frustrated by medical jargon, but when it is not used they feel better and more able to focus on recovery. Only $57 \%$ of hospitalised children above the age of 12 understand the information doctors give them regarding their care and treatment (Commission for Healthcare Audit and Inspection, 2007).

Objective

To create a teaching resource for healthcare professionals that will increase awareness and use of child-friendly language, and avoidance of medical jargon, and increase users' confidence in paediatric communication.

Methods

Dr Jargon Paediatrics, a teaching resource in the form of a card game, was created to address this need. Players must practise describing diseases, treatments and procedures to each other against a timer while avoiding medical jargon. Focus groups of healthcare professionals and students played the game and completed questionnaires before and after.

Results

The first focus group completed questionnaires before playing, rating their confidence in explaining a medical term to a child: of 13 participants, $69 \%$ reported feeling 'slightly confident' and $31 \%$ 'not at all confident'; none selected 'very confident' or 'confident'. All three focus groups completed questionnaires after playing: of 45 participants, $5 \%$ agreed and $95 \%$ strongly ageed that the game was a useful resource for practising jargon-free paediatric communication. One hundered percent of participants reported that more teaching on giving medical information to children is needed in training programmes.

\section{Conclusion}

Dr Jargon Paediatrics is a 'fun and innovative' resource which builds awareness and confidence in paediatric communication and avoiding medical jargon. It has now been commercially developed and has gained further positive feedback from users as well as press coverage.

659

\section{Primary Care}

The Dogma That Children Are Therapeutic Orphans Challenged Klaus Rosel

${ }^{T}$ Medical Science, klausrose Consulting, Switzerland

\section{Background}

In 1963, Shirkey described children as "therapeutic orphans" (TOs), claiming they were denied the use of many new drugs. The American Academy of Pediatrics (AAP) took this up; resulting in US pediatric legislation (USPL) and eventually EU pediatric legislation (EUPL). Since 2007, new drugs cannot be EU-registered without pediatric investigation plan (PIP).

Objective

To verify/challenge Shirkey's, AAP's, USPL's and EUPL's assumptions Methods

Shirkey's original publication, AAP guidelines on pediatric studies \& offlabel, and the 1962 US law were analyzed. Key assumptions and the rationale for demanding separate pediatric safety \& efficacy (S\&E) studies were examined against the history of medicine $\&$ pediatric sub-disciplines.

\section{Results}

Shirkey incorrectly used "children" for underage persons as both a legal and physiological term. He misinterpreted some unintended consequences of the US law. Drug label warning clauses "not to be used in children" were not legally binding; they protected companies against lawsuits. In 1995, the AAP alarmingly "proved" that differences from adults in "kinetics, end organ responses, and toxicities" of drugs in children of all ages by citing two papers on preterm newborns. Children profit(ed) even more from modern drugs than adults. Pediatric oncology, pediatric cardiology, neonatology evolved successfully using drugs off-label. Children usually need assessment of appropriate dosing, not separate S\&E studies. Children are not another species. Young children differ considerably from adults, but not adolescents in whom adult doses are appropriate as demonstrated by 2016 FDA labels for antiepileptic/ anticancer drugs.

\section{Conclusion}

The TO concept and demands for separate pediatric S\&E studies are based on misinterpretation/confusion at the interface of law and medicine. Use of drugs in adolescents needs legal fixing, not S\&E studies. Younger children need dose confirmation after modeling \& simulation. The EAP needs an independent position on drug development and off-label use and should distance itself from the PIP system.

\section{1}

\section{Primary Care}

Point-of-care Ultrasound Performed By Pediatric Provider in a Community Setting Practice

Adib Salim $^{1}$, Marina Balestra ${ }^{2}$, Rino Agostiniani ${ }^{3}$

${ }^{T}$ Pediatrician, ATS, Italy

${ }^{2}$ Pediatrician, ATS, Italy

${ }^{3}$ Pediatrician, ATS, Italy

\section{Background and aims}

Bedside UltraSound is a safe technology with numerous applications and can be performed parallel to physical examination for rapid assessment of ill children presenting a variety of symptoms.

\section{Objective}

To assess UltraSound application as an important modality to reinforce clinical diagnosis and decision-making and cost containing in the context of the community system of care.

\section{Material and method}

Observational review study of our experience in pediatric ambulatory setting practice: a total of 3.250 US examinations were performed between years 2000 and 2015.

In this study we review our results to determine the benefits of US technique in pediatric patients presenting with wide spectrum of clinical conditions: in acute illness, late detection of congenital anomalies among infants and follow up of repaired and unrepaired heart conditions.

\section{Conclusions}

Despite challenges associated with integrating point of care US into pediatrics, major efforts should be done to make more pediatricians familiar with diagnostic US tool and give them sufficient skills in their workplace setting practice.

UltraSound system are becoming more affordable and easy to use, however, US results depend on the operator's training and experience.

Appropriate training and educational programs are mandatory to ensure that many physicians: can accurately perform and interpret bedside US examination and learn how to incorporate US findings in the clinical management of children.

Authors

Dr Adib Salim ${ }^{(1)}$, Dr Marina Balestra ${ }^{(1)}$, Rino Agostiniani ${ }^{(2)}$

- PDF ATS Bergamo - Italy

- PDF ATS Pistoia - Italy 
80

\section{Primary Care}

Post Traumatic Stress Disorder (ptsd) among Pediatric Residents at the Philippine Children's Medical Center (pcmc)

Jesus Nazareno Velasco ${ }^{1}$, Bernardus Andrew Tirta ${ }^{1}$

${ }^{T}$ Pediatric ICU, Philippine Children's Medical Center, Philippines

\section{Background}

Presently, there are no studies on the prevalence of PTSD among healthcare professionals in the Philippines. PTSD may contribute to poor performance and poor interpersonal relationship in the workplace.

Objectives

To determine the prevalence of PTSD among our pediatric residents and to identify external stressors and internal vulnerabilites predisposing to PTSD.

Methods

The Institutional Review Board approved this protocol. Informed Consent Forms were obtained. The study design was cross-sectional. All pediatric residents from the 1 st to the 3 rd year levels were recruited. All answered the PTSD Checklist Civilian Version questionnaire. Their demographic profiles, external stressors (clinical areas of rotation, hours of sleep), and internal vulnerabilities (past personal history of depression, suicidal ideation, subjective perception of difficulty) were recorded. Frequencies, percentages, and Pearson Correlation Analysis were computed.

Results

Sixty pediatric residents paticipated. $16.7 \%$ showed symptoms of PTSD. Among the PTSD-symptomatic residenrts, $90 \%$ were females; $40 \%$ belonged to the 1 st year level, and another $40 \%$ to the 3 rd year. The most stressful clinical area identified was the Emergency Room (40\%) and $50 \%$ had only less than 3 hours of sleep on their 24-hour tour of duty. Furthermore, $20 \%$ had personal history of depression and suicidal ideation. None of these PTSD-symptomatic were under the care of a psychiatrist and only $30 \%$ of them were willing to seek professional help. These stressors and vulnerabilities were not significantly correlated to the development of PTSD symptoms.

\section{Conclusion}

The prevalence of probable PTSD in this study was high. No single most reliable stressor nor vulnerability seemed to predict PTSD. Multiple factors most probably come into play over time to produce PTSD symptoms. The high prevalence of probable PTSD in this study is a reason for great concern. Added to the seriousness of the matter is the fact that none of them were under the care of a psychiatrist and only $30 \%$ were willing to seek professional help.

716

\section{Primary Care}

Effects of Watching Cartoons on Pain in Children during Venipuncture

Esra Ardahan', Yakup Karahan ${ }^{1}$, Funda Saime Basoglu', Aysel Ogul ${ }^{2}$, Beste Oztornaci ${ }^{1}$, Perihan Yetim ${ }^{2}$, Hatice Yildirim Sari

${ }^{1}$ Pediatric Nursing Department, İmir Katip Celebi University Faculty of Health Science, Turkey

${ }^{2}$ Pediatric Department, Tepecik Education and Research Hospital, Turkey

\section{Background}

Pain is an unpleasant sensory and emotional experience accompanying existing or potential tissue damage or described with this damage. Many children experience pain and may become restless during invasive procedures. Pain-relieving methods during invasive procedures are generally classified as pharmacological and non-pharmacological methods. Nurses frequently employ nonpharmacological methods in pain management.

Objective

The aim of this study was to determine the effects of watching cartoons on pain scores and duration of crying during venipuncture.

\section{Methods}

The study included 81 36-72-month-old children who presented to the blood collection unit of the childrens clinics. Their children watched a cartoon then the venipuncture process was initiated. The children's pain levels and the duration of crying were assessed during the venipuncture. After the venipuncture, they continued to watch the cartoon and their pain levels were assessed again. During the process; another researcher calculated the children's duration of crying. The children in the control group were subjected to the same process but were not shown the cartoon.

Results

Significant differences were determined between the groups in terms of pain levels, duration of crying after the procedure and total duration of crying. There was no significant difference between the two groups in terms of duration of crying during the procedure.

\section{Conclusions}

Watching cartoons, one of the non-pharmacological pain relieving methods, reduces perception of pain by distracting a child's attention.

774

Respiratory

Lung Ultrasound in Early Diagnosis and Differentiation between Bacterial and Viral Pneumonia in Egyptian Infants Ahmed Omran ${ }^{1}$, Heba Awad ${ }^{1}$, Mostafa Ibrahim ${ }^{2}$, Sonya El-Sharkawy ${ }^{I}$ ${ }^{7}$ Department of Pediatrics \& Neonatology,, Faculty of Medicine, Suez Canal University,, Egypt

${ }^{2}$ Department of Radiodiagnosis, Faculty of Medicine, Suez, Canal University, Egypt

\section{Background}

Lung ultrasound (LUS) is an extremely valuable diagnostic tool in detecting pneumonia in children of all ages. However, limited data have been published showing its effectiveness in early differentiation between bacterial and viral pneumonia in infants.

Objective

To evaluate the applicability of LUS in early diagnosis and differentiation between bacterial and viral pneumonia in Egyptian infants.

\section{Methods}

LUS was performed in 52 infants presented with clinical signs suggesting of community acquired pneumonia (CAP) within the first 6:12 hours of admission in Pediatric department of Suez Canal University, Ismailia, Egypt.

\section{Results}

Among the 52 infants included in the study, $65.3 \%$ were diagnosed to have bacterial pneumonia and $34.6 \%$ diagnosed to have viral pneumonia. In bacterial pneumonia, Subpleural consolidation $1 \mathrm{~cm}$ and air bronchogram were the most prominent features with statically significant relationship. In viral pneumonia multiple B lines and subpleural consolidations $1 \mathrm{~cm}$ were the most prominent features with statically significant relationship.

\section{Conclusion}

We found LUS to be a reliable and non-invasive tool for the early diagnosis and differentiation between bacterial and viral pneumonia in infants.

356

Respiratory

Risk Factors for Severe Bronchiolitis: a Retrospective Review of Patients Admitted to the University Hospital in Central Region of Slovenia

Ajda Praznik ${ }^{l}$, Neža Vinšek ${ }^{l}$, Ana Prodan ${ }^{l}$, Štefan Grosek ${ }^{2}$, Vanja $\overline{\text { Erčulj }^{5}, \text { Marko Pokorn }}{ }^{3}$, Darja Paro ${ }^{2}$, Tatjana Mrvič ${ }^{3}$, Uroš Krivec ${ }^{2}$, Franc Strle ${ }^{3}$, Miroslav Petrovec ${ }^{4}$, Marta Žnidaršič Eržen ${ }^{2}$

${ }^{1}$ Faculty of Medicine, University of Ljubljana, Slovenia

${ }^{2}$ Paediatrics, University Medical Centre Ljubljana, Slovenia

${ }^{3}$ Paediatrics, Clinic for Infectious Diseases, University Medical Centre Ljubljana, Slovenia 
${ }^{4}$ Laboratory for Diagnostics of Viral Infections, Institute of Microbiology and Immunology, Slovenia

${ }^{5}$ Statistics, Ro sigma, Slovenia

\section{BACKGROUND}

Bronchiolitis is a potentially life-threatening respiratory infection that affects children. Viral infections of the lower respiratory tract represent a global problem.

\section{OBJECTIVE}

The objective of our study was to determine the microbiological and clinical factors associated with the severity of bronchiolitis.

\section{METHODS}

A retrospective chart review of children 2 years old, diagnosed with bronchiolitis at the University Medical Centre Ljubljana (UMCL) between 1. 5. 2014 and 30. 4. 2015, who were treated as outpatients (PED group) or as inpatients in the standard hospital setting (WARD group) or in the intensive care unit (PICU group), was performed. Detection of several respiratory viruses in nasopharyngeal swab in a subgroup of patients was accomplished by RT-PCR. RESULTS

The study included 761 children. The most frequently detected viruses were respiratory syncytial virus (RSV), human rhinovirus (hRV) and human bocavirus (hBoV). Patient groups (PED, WARD, PICU) differed in Wang respiratory score for the severity of bronchiolitis (p0.001). No statistically significant differences in causative viruses were found comparing the three groups. There was a significantly lower proportion of children with the presence of more than 1 virus in nasopharyngeal swab, in patients who needed treatment in PICU compared to PED and WARD groups $(p=0.017)$. The three groups statistically significantly differed in age and birth weight, proportion of patients with comorbidities, treatment with bronchodilator and use of antibiotics. However, multiple regression analysis revealed that only younger patient age and the use of antibiotics were associated with a more severe disease course.

CONCLUSION

$\mathrm{RSV}, \mathrm{hRV}$ and $\mathrm{hBoV}$ were the most frequently detected viruses in our patients with bronchiolitis. The majority of bronchiolitis patients admitted to the PICU had only one virus detected. Younger age and the use of antibiotics were associated with a more severe course of the disease.

250

Rheumatology

Increased levels of plasma pentraxin 3 predict IVIG resistance and coronary artery lesion formation in patients with Kawasaki disease Toshiyuki Kitoh $^{1,2}$, Reizo Baba ${ }^{2,3}$, Yoshiro Kitagawa ${ }^{2}$, Taichiro Muto ${ }^{2}$, $\overline{\text { Akihisa Okumura }}{ }^{2}$, Noriaki Kume ${ }^{4}$, Takao Hamakubo ${ }^{5}$

${ }^{1}$ Department of Pediatric Pharmacology, Aichi Gakuin University, School of Pharmacy, Japan
${ }^{2}$ Department of Pediatrics, Aichi Medical University, School of Medicine, Japan

${ }^{3}$ Department of Lifelong Sports and Health Sciences, Chubu University College of Life and Health Sciences, Japan

${ }^{4}$ Division of Clinical Pharmacy, Kobe Gakuin University, Faculty of Pharmaceutical Sciences, Japan

${ }^{5}$ Department of Quantitative Biology and Medicine, Research Center for Advanced Science and Technology, the University of Tokyo, Japan

\section{Background}

Kawasaki disease (KD) is a systemic vasculitis and childhood febrile disease that can lead to cardiovascular complications. Delaying definitive diagnosis of KD and intravenous immunoglobulin (IVIG) or specific treatment leads to development of coronary artery lesion (CAL). However, there are no specific biomarkers for KD definitive diagnosis in clinical settings. Objective: To determine whether novel biomarkers for endothelial cell damage are accurate markers of inflammation in $\mathrm{KD}$, and whether plasma levels of these markers correlate with resistance to initial IVIG and the development of coronary artery lesions (CAL) in KD.

\section{Methods}

In this prospective study of 97 children with $\mathrm{KD}$ ( 75 complete KD and 22 incomplete KD, 94 without $\mathrm{CAL}$ and 3 with $\mathrm{CAL}$ ), recurrence 3 cases IVIG resistant 22 , IVIG responsive; 63 cases, no IVIG; 12 case. Plasma pentraxin 3 (PTX3), soluble lectin-like oxidised low-density lipoprotein receptor-1 (sLOX-1), and matrix metalloproteinase-9 (MMP-9) concentrations were analysed at three clinical points: (a) up to IVIG treatment, (b) after IVIG treatment, and (c) during convalescence after fever resolution and compared with linical outcome; IVIG resistance and transient or ultimate CAL fromation detected by Echocardiogram.

\section{Results}

Increases in levels of plasma PTX3 were only significantly higher compared with non-KD febrile controls. PTX3 level was significantly higher in IVIG non-responders than in IVIG responders ( $\mathrm{p} 0.001$ ). PTX3 level was significantly correlated with the number of courses of IVIG treatment needed to resolve fever. ( $p$ 0.001). The patients with CAL had higher PTX3 levels than patients without CAL $(85 \pm 8.4$ vs. $22 \pm 2.2 \mathrm{ng} / \mathrm{ml}, \mathrm{p} 0.0001$ ). The cut-off value of $69 \mathrm{ng} / \mathrm{ml}$ to predict CAL formation had a specificity of 1.00 , sensitivity of 0.98 , and likelihood ratio of 49.0 .

\section{Conclusions}

Levels of PTX3 were correlated with the severity of Kawasaki disease. A PTX3 level above $69 \mathrm{ng} / \mathrm{ml}$ predicted CAL formation. These data suggest that PTX3 can be a definitive biomarker for prediction of IVIG resistance and subsequent CAL formation in patients with Kawasaki disease 


\section{Poster Presentations}

497

Adolescent Medicine

Growth and nutritional status of adolescents school Jane Cardoso $^{1}$, Jane Laner Cardoso ${ }^{1}$, Claudio Leone ${ }^{2}$

${ }^{T}$ Public Health/Pediatrician, University Center of Maringá, Brazil

${ }^{2}$ Public Health, University of São Paulo, Brazil

\section{Objective}

To evaluate the stature growth achieved and the nutritional status of adolescents from public schools in a metropolitan area of the Southern Region of Brazil.

\section{Methods}

A cross-sectional study of a convenience sample of 7225 schoolchildren, $47.8 \%$ girls and $51.3 \%$ boys, 10 to 16 years of age. Stature (E), body weight and Body Mass Index (BMI) were evaluated according to Norms of the Ministry of Health (MS), and the values were transformed into z scores by the World Health Organization (WHO 2007) reference framework, comparing Their central trends and their variability. Classification of nutritional status and growth was by WHO/MS criteria.

\section{Results}

The highest proportion of adolescents was 10 years old (37.4\%), decreasing year by year, the lowest being 15 and 16 years old $(4.4 \%)$. The mean $\mathrm{z}$ score of $\mathrm{E}$ decreases progressively from 0.1 to -0.4 with age; With standard deviations (dp) ranging from 1.2 to 1.0. The mean $\mathrm{Z}$ score of BMI also decreases from 0.5 to 0.3 (girls) and 0.6 to 0.3 (boys), with dp varying from 1.4 to 1.2. Regarding the short stature (BE) and very short stature (BEM) the prevalences were 2.6 and $0.6 \%$, respectively, without difference by sex. The prevalences of overweight (SP), obesity $(\mathrm{Ob})$ and severe obesity $(\mathrm{ObG})$ were $20.3 ; 10.0$ and $1.7 \%$, with boys presenting significantly higher prevalences of $\mathrm{Ob}$ and $\mathrm{ObG}$. BMI $\mathrm{z}$ scores also showed a decreasing trend with age. Slight thinness and thinness were rare, affecting 1.8 and $0.5 \%$ of the sample. Conclusions

Adolescents from public schools in the south of the country have a high prevalence of overweight, obesity and severe obesity, and show an average growth similar to that expected, despite a decreasing trend with age. Table which is compatible with an advanced phase transition.

\section{0}

\section{Adolescent Medicine}

Diagnostic Difficulties in Adolescents Presenting with Polyserositis Alexandra Coroleuca ${ }^{1,2}$, Virginia Sobek ${ }^{1}$, Andreia Nita ${ }^{1,2}$, Raluca Maria Vlad $^{1,2}$, Dumitru Oraseanu ${ }^{I, 2}$, Daniela Pacurar ${ }^{1,2}$

${ }^{1}$ Pediatrics, "Grigore Alexandrescu" Emergency Hospital for Children, Romania

${ }^{2}$ Medicine, "Carol Davila" University of Medicine and Pharmacy, Romania

\section{Background}

Polyserositis is defined as the inflammation of serous membranes associated with serous effusion. Differential diagnosis is often difficult because polyserositis occurs in various conditions. It is frequently associated with tuberculosis (TB), autoimmune diseases, tumors, rarely with sepsis or other infections.

\section{Objective}

The aim of this paper is to highlight the necessity of a diagnostic protocol for children presenting with polyserositis.

\section{Methods}

We present the cases of two patients admitted to our hospital with pleural and peritoneal effusion who were diagnosed with Non-Hodgkin intestinal lymphoma.

Results

A 12 year-old girl was admitted to our department after 5 days history of fever, cough, chest pain, abdominal pain and weight loss. Laboratory tests showed high inflammatory markers and the chest X-ray was suggestive for bilateral pleural effusion. TB was considered for the differential diagnosis, but in was excluded. CT scan showed massive pleural and peritoneal effusion with gastric distention. Exploratory laparoscopy revealed a compressive mass in the duodeno-jejunal angle with abnormal aspect of the intestinal loops, fibrin deposits and multiple adherences. Excisional biopsy was performed and the diagnosis of anaplastic lymphoma was established (histology and immunohistochemistry).

A 15 year-old boy was transferred to our hospital with the suspicion of liver disease. The patient had a history of abdominal pain, vomiting and abdominal distension with onset of 4 days. Abdominal ultrasound identified peritoneal and pleural effusion and an abdominal mass. Laparotomy showed peritoneal carcinomatosis, large intestinal tumor $30 \mathrm{~cm}$ before the ileo-cecal valve. After assessment of biopsies, Burkitt Lymphoma was diagnosed.

Both patients were started on specific treatment, but the second patient had a fulminant evolution and died in the 18th day of admission.

\section{Conclusion}

Polyserosistis can present as a complication of lymphomas. The diagnosis should be considered even in case of sudden onset. A suitable assessment should be made to establish the accurate diagnosis.

\section{8}

\section{Adolescent Medicine}

Mobile phone call impact on children and adolescents health: keynote remarks

$\underline{\text { Styliani Geronikolou }}^{1,2}$, Dennis $^{2}$ Cokkinos $^{1}$, George Chrousos $^{2}$, Christina Kanaka-Gantenbein $^{2}$

${ }^{I}$ Clinical, Translational, Experimental Surgery, Biomedical Research Foundation of the Acaemy of Athens, Greece

${ }^{2}$ First Clinic of Pediatrics, Athens University, Aghia Sophia Children Hospital, Greece

\section{Background}

Radiofrequency fields' (RF) exposure of fetuses, infants, children and adolescent is intense, unambiguous and inevitable.

\section{Aim}

To outline all evidence relative to children and adolescents and reveal influences by RF (mobile phone) exposure.

\section{Methods}

Systematic review (and meta-analysis where possible) of literature related to RF description and or its impact on all body functions.

\section{Results}

EFHRAN identified two types of exposure sources: body close sources and far body sources. The $63 \%$ of the total radio- exposure that a person is exposed to is the mobile phone (main body-close source). Total exposure is inadequately or insufficiently described in literature but seems to play a crucial role in the effects. A review of 2743 studies identifies 7 groups of influences (carcinogenic, genotoxic, circadian rhythms, neural, reproductive, endocrine, addiction) and six physiological mechanisms (apoptosis, heat, resonance, oxidative stress, hydrophilic head or hydrophobic tails and/or calcium channels alterations in cell membrane). The body responds to electromagnetic fields by changes in all body's functions. Although, the reported effects on endocrine system are multiple (thyroid, HPA, reproductive, somatotrophic etc.), the reporting quality made metaanalysis impossible in the field. On the contrary, meta-analytic results include increase in dynamic and complexity of cardiac function (LLE $\mathrm{g}=1.408$, CI95\% [0.334, 0.408], SD1 $\mathrm{g}=0.659$, CI95\% $[-0.243$, -3.107]) during a mobile phone call. Furthermore, meta-regression results showed (i) strong vulnerability in the autonomic nervous system of the adolescents $\mathrm{QM}(1)=4.378, p=0.03, \mathrm{QR}(2)=4.24$ $p=0.12$ and (ii) the duration of a mobile call does not affect heart rate variability or the sympathovagal balance. 


\section{Conclusions}

There is strong evidence that the pediatric research should orientate towards RF impact on children body functions.

\section{4}

\section{Adolescent Medicine}

Cardio-vascular Profile of Greek Adolescents with Polycystic Ovary Syndrome

Styliani Geronikolou ${ }^{1,2}$, Flora Bacopoulou ${ }^{1,2}$, Stavros Chryssanthopoulos ${ }^{2}$, Dennis Cokkinos

${ }^{1}$ Center for Adolescent Medicine and UNESCO Chair on Adolescent Health Care, First Department of Pediatrics, School of Medicine, National and Kapodistrian University of Athens, "Aghia Sophia" Children's Hospital, Greece

${ }^{2}$ Clinical, Translational and Experimental Surgery Research Centre, Biomedical Research Foundation of Academy of Athens, Greece

\section{Aim}

The aim of this case-control study was to examine potential subtle changes in cardiac and vascular function of adolescent girls with polycystic ovary syndrome (PCOS).

\section{Methods}

Nineteen PCOS patients and eighteen body mass index (BMI) matched non-PCOS controls, aged 13 to 21 years, underwent assessment of (i) baroreflex sensitivity (BRS) as marker of cardiac autonomic system, (ii) pulse wave velocity (PWV) as marker of arterial stiffness (iii) intima media thickness (IMT) as marker of arterial thickness, (iv) abdominal adipose tissue (AAT) as marker of atherosclerosis and metabolic syndrome, (v) phase angle $(\mathrm{PhA})$ as marker of inflammation.

Results

Non parametric analysis showed statistically significant differences in PWV $(p=0.005)$ biomarker between PCOS patients and controls. No differences were detected in any other marker studied herein.

Conclusions

Study results showed early onset of vascular dysfunction and predisposition to hypertension in adolescent PCOS.

\section{4}

\section{Adolescent Medicine}

Drug-induced acute liver failure in children

Alina Grama', Aurel Bizo ${ }^{1,2}$, Dan Delean ${ }^{2}$, Cornel Aldea ${ }^{2}$, Bogdan Bulata', Tudor L Pop

${ }^{1}$ Pediatric Gastroenterology, 2nd Pediatric Clinic, University of Medicine and Pharmacy "Iuliu Hatieganu" Cluj-Napoca, Romania, Romania

${ }^{2}$ Pediatric Nephrology, 2nd Pediatric Clinic, University of Medicine and Pharmacy "Iuliu Hațieganu” Cluj-Napoca, Romania, Romania

\section{Introduction}

Acute liver failure (ALF) is a syndrome characterized by jaundice, coagulopathy (INR 1.5) and hepatic encephalopathy in patients with no evidence of prior liver disease. Liver injury due to toxins and drugs is one of the most common aetiology of ALF in children.

Methods

In the last years, in our country the drugs ingestion is growing especially among adolescents. Methods: We have analysed retrospectively all patients who developed ALF after drugs exposure, hospitalized between January 2012 and December 2016 in our hospital, the main Toxicology Centre in Nord-Western Romania.

Results

During the last 5 years the number of drug-induced ALF increased dramatically: 20 cases (one case in 2012, two cases in 2013, three cases in
2014, four cases in 2015 and 10 cases in 2016). The most common drugs that caused toxic hepatitis with ALF were: acetaminophen (16 cases), albendazole ( 3 cases), valproate ( 2 cases) and colchicine (one case). Fortunately, the use of acetylcystein as an antidote in acetaminophen intoxication (one of the most used drug for suicide among teenagers in our region) makes mortality among these cases to remain low. Only one patient died: a teenager who ingested voluntary sixty colchicine tablets.

\section{Conclusions}

Unfortunately, in the last 5 years the incidence of teenagers who used drugs for suicide purposes is on the rise in our area. The most commonly used is acetaminophen due to the easy access to it, being released without medical prescription. Many of these drugs used in excess are toxic for the liver and in the severe cases can cause ALF or death.

101

Adolescent Medicine

Impact of Reproductive Health Education on the Knowledge of Mid Adolescents Boy of Urban Population of India Gaurav Jadon ${ }^{l}$

${ }^{T}$ Pediatrics, NMC SPECIALITY Hospital, United Arab Emirates

Overall objective was to study impact of reproductive health education on knowledge of mid-adolescents boys of urban population with special reference to reproductive health.

\section{Study design}

The study was cross-sectional type conducted over a time period of six months.

\section{Methods}

The study subjects were drawn from three schools, of urban population of Faridabad, in Haryana. The present study was designed to evaluate the existing knowledge and impact of reproductive health education amongst mid-adolescent boys, related to adolescent health with special reference to secondary sexual characteristics, menstrual cycle, pregnancy and motherhood, AIDS and family planning practices.

\section{Sample Size}

A sample size of 250 students was selected for this study. Study group: comprised of mid adolescent boys and girls of 14-16 years, studying in co-educational senior secondary schools. Data from students was collected using a structured self-administered questionnaire.

\section{Conclusion}

There was marked improvement in knowledge of same adolescents after education on the reproductive health in almost each and every field.

The fact that children engage in sexual behavior before they have a clear understanding of what it is all about, places them at very high risk of a variety of adverse experiences that can impact negatively on their development. Adolescents whom mothers were housewives were more aware of their reproductive health as compare to working parents. But parent's education doesn't produces significant difference in their knowledge. Conversely, although many adolescents have sexual knowledge, this knowledge does not always influence their behavior.

\section{3}

\section{Adolescent Medicine}

Relationship between low-grade inflammation and arterial hypertension at obese children Nelea Mătrăgună ${ }^{1}$, Svetlana Cojocari ${ }^{1}$, Bichir-Thoreac Lilia ${ }^{l}$ ${ }^{T}$ Cardiologycal Institute Republic of Moldova

\section{Background}

Obesity is characterized by a state of low-grade inflammation at all ages. Body fat correlates with the different components of the metabolic syndrome (MS). There is evidence to show that chronic subclinical inflammation in childhood is associated with metabolic dysfunction, which 
can lead to insulin resistance and the MS. The aim of the study was: To investigate the relationship between inflammatory parameters and arterial hypertension $(\mathrm{AH})$ in obese children and to elucidate their effects on the development of MS.

\section{Methods}

The investigation included 218 children with obesity/overweight at the age of 10-18 (108 (49,54\%) hypertensive children and $110(50,46 \%)$ normotensive children), 46 of children $(21,10 \%)$ - met the criteria for the MS. We determined TNF $\alpha$, hsCRP, lipid profile and serum insulin. MS was diagnosed according to the IDF criteria.

\section{Results}

Children with AH had significantly higher levers of inflammatory markers as compared with normotensives. But the highest values of hsCRP $(3,7 \pm 0,14$ vs $2,3 \pm 0,08 \mathrm{mg} / \mathrm{l} ; \mathrm{p} 0,001)$ and $\mathrm{TNF} \alpha(13,1 \pm 0,68$ vs $7,5 \pm 0,21 \mathrm{pg} / \mathrm{ml} ; \mathrm{p} 0,001)$ were found at children with MS as compared with the ones without it. hsCRP and TNF $\alpha$ has been correlated positive, statistically significant, with BMI ( $\mathrm{r}=+0,71, \mathrm{p} 0,001$ and, respectively, $\mathrm{r}=+$ $0,75, \mathrm{p} 0,001), \mathrm{AC}(\mathrm{r}=+0,75, \mathrm{p} 0,001$ and respectively, $\mathrm{r}=+0,75, \mathrm{p} 0,001)$, tension values $(\mathrm{r}=+0,63 \mathrm{p} 0,001$ and, respectively, $\mathrm{r}=+0,55, \mathrm{p} 0,001)$, total cholesterol ( $\mathrm{r}=+0,44, \mathrm{p} 0,001$ and, respectively, $\mathrm{r}=+0,43$, p0,001), LDL-C $(\mathrm{r}=+0,55, \mathrm{p} 0,001$ and, respectively, $\mathrm{r}=+0,52, \mathrm{p} 0,001)$, triglycerides $(\mathrm{r}=+$ $0,47, \mathrm{p} 0,001$ and, respectively, $\mathrm{r}=+0,45, \mathrm{p} 0,001)$, serum insulin $(\mathrm{r}=+0,67$, $\mathrm{p} 0,001$ and, respectively, $\mathrm{r}=+0,69, \mathrm{p} 0,001)$ and negative with HDL-C $(\mathrm{r}=-0,62 \mathrm{p} 0,001$ and, respectively, $\mathrm{r}=-0,56, \mathrm{p} 0,001)$.

Conclusion

Our results suggest that overweight is implied in the modification of the proinflammatory parameters. Higher rate of these disorders at hypertensive children, and the ones diagnosed with MS denotes the fact that low grade inflammation can make a pathogenic chain between obesity, AH and MS.

\section{0}

\section{Adolescent Medicine}

Endothelial Dysfunction Corection in the Teenagers with Primary Artherial Hypertension by Essential Phospholipids

Horlenko Olesya ${ }^{2}$, Vasil Rusyn ${ }^{1}$, Natalia Sochka ${ }^{2}$, Debretseny Olena ${ }^{2}$, $\overline{\text { Horlenko Fedir }}^{1}$, Radovetska, Galina ${ }^{2}$, Alekszandrov Alekszandr ${ }^{2}$, Pushkarenko Olga ${ }^{2}$, Kossey Gabriella ${ }^{2}$

${ }^{1}$ Surgical Diseases, Uzhhorod National University, Medical Faculty, Ukraine

${ }^{2}$ Pediatrics with Infectious Diseases, Uzhhorod National University, Medical Faculty,, Ukraine

\section{Backgruond}

Formation of arterial hypertension (PAH) is associated not only with the state of vascular tone, but it is associated with endothelial relaxation disorders. We investigated morphological features of PAH duration with endothelial dysfunction (ED) in the children by used optimized treatment Material and methods

The 80 children (15-17 years) diagnosed with PAH ED were examined and divided into two groups: 1 group, $n=40$ patients, used basic treatment), group 2, $n=40$ patients, who used optimized treatment (with the lecithin inclusion to the basic treatment in the age dose).

\section{Results}

Problems with homeostasis were found in the case of adolescents with $\mathrm{PAH}$ and ED in the endogenous antioxidant system, the level of intensity of child's organism inflammatory response.Based on cytokine profile tests and the antibody production of child's organism, the morphofunctional characteristics of the heart and blood vessels, the clinical and pathogenetic significance of ED of adolescents with PAH were confirmed. The efficiency of optimized treatment has been proved in the case of adolescents with PAH and ED when they used basic treatment with the inclusion of essential phospholipids(lecithin).

\section{Conclusion}

Levels of aortic stiffness index tended to decrease, as confirmed by improving hemodynamic parameters. Value Ve/Va had direction to increase. Vessel vasoconstriction was significantly decreased during the reactive hyperemia test, but the degree of vasodilation was varied depending from the method of treatment. KIM thickness decreased to 1.12 times in the children with optimized treatment along with a rate decrease in the 2 times of the endothelial cell desquamation.

\section{8}

Adolescent Medicine

Childhood Experiences of Physical, Emotional and Sexual Abuse among medical Students in Cairo University

Nazih Ramadan ${ }^{1}$

${ }^{T}$ forensic medicine and clinical toxicology, Faculty of medicine, Cairo university, Egypt

\section{Background}

Child maltreatment includes five major subtypes: physical, sexual, and emotional abuse; neglect; and exposure to intimate partner violence. The more the individual is exposed to abuse and neglect and the greater the tendency toward this among those around them, the greater the probability of the victim subsequently abusing others. It should also be considered that kids who experienced abuse and neglect during childhood are at higher risk for some dramatic consequences such as attention deficiency, hyperactivity, substance misuse, high-risk sexual activity, and personal identity problems. These significantly affect the child not only during childhood but also in the life period that follows

Aim of the study

Was to obtain the prevalence of childhood abuse experiences among medical Students and if this occurs now. Also it can give an indication about scientific achievement as the medical students are mostly the most intelligent in the country.

\section{Methodology}

This cross-sectional study was performed on 248 students who were randomly selected from the 4th year medical students at faculty of medicine Cairo University. They were asked to answer a preformed questionnaire after giving informed consent. They were asked about demographic data, exposure to different types of child abuse and if still exposed or not.

Results

From 248 participants, 99 were males and 149 were females. About $48 \%$ of total sample memorized exposure to childhood abuse. Thirty-nine percent memorize exposure to both emotional and physical abuse, $5 \%$ of them experience additional sexual abuse. Emotional exposure alone was $2 \%$ and physical alone was $7 \%$. About $27 \%$ of the abused are still physically or emotionally abused till now. Memorization of different types of abuse is nearly equal for both males and females with males slightly higher.

Conclusion

Different types of child abuse are present in Egypt, and it can be memorized till adolescence age, further studies about its effects on educational level should be conducted.

273

\section{Adolescent Medicine}

From reading to healing: managing psychological tension in hospitalized children through therapeutic stories

Pelagia Tsitsani ${ }^{1}$, Vasiliki Blogoura ${ }^{1}$, Katerina Tzortzakaki ${ }^{2}$, Vasiliki Moschou ${ }^{3}$

${ }^{1}$ Paaediatric Clinic, General Hospital of Didimoteicho, Greece

${ }^{2}$ Author, Psychologist, Greece

${ }^{3}$ Private Practice, Paediatrician, Greece

\section{Introduction}

In the course of hospitalization, children face emotions such as fear, separation, loneliness, in addition to physical weakness - immobility due to their disease, intravenous lines etc. Parents have to manage the responsibility of decisions and maybe even some personal guilt, and are 
therefore not giving the children the chance to express how they feel. Healthcare professionals may lack adequate training and time to cover such needs.

\section{Purpose}

Each "difficult" situation offers a chance to learn, if freedom of expression is guaranteed. Our purpose was to examine whether reading stories written by a psychologist can help children adjust to hospital environment.

\section{Materia}

The project was carried out during the last trimester. The participants were 32 children, aged 8-16 years old, who were hospitalized for at least 5 days and were receiving intravenous treatment. The children were given stories to read, followed by discussion and expression through theatrical games and drawing.

\section{Method}

A qualitative research method was applied. One researcher carried out interviews - discussions with each child, using eight open questions as a guide. The other researcher observed and noted down everything (even the pauses). This was followed by activities based on the story. Data was codified and three themes emerged.

\section{Outcome}

The participants enjoyed reading and responded enthusiastically to the activities. Younger children expressed themselves more through drawing and sketching, whereas older ones preferred to impersonate the heroes of the stories and engage in dramatic play.

Conclusions

A child's hospitalization can be an emotionally learning and evolving experience, given the proper support.

259

\section{Adolescent Medicine}

Investigation of the Relationship Between Depression Symptoms and Related Factors of High School Students

Gulcin Yapici $^{1}$, Hakk Aktas ${ }^{1}$, Fazıl Kocaş ${ }^{1}$, Coşar Uzun ${ }^{2}$, Fatih Tekin ${ }^{3}$, Nurten Erdal

${ }^{1}$ Department of Public Health, Mersin University Faculty of Medicine, Turkey

${ }^{2}$ Department of Biophysics, Mersin University Faculty of Medicine, Turkey

${ }^{3}$ Department of Public Health, Necmettin Erbakan University Faculty of Medicine, Turkey

\section{Background}

The frequency of psychological diseases in adolescents has increased due to changes in social, cultural and family values in recent years.

\section{Objective}

To investigate the frequency of depression symptom in high school students, the level of electromagnetic field (EMF) in school environment and other related factors.

\section{Methods}

This cross-sectional study was determined of the high school in a district area of Mersin. The research population consisted of 7791 students. Minimum sample size was calculated 558 students and 605 questionnaires were evaluated. The questionnaire was including the sociodemographic characteristics of the students, and the Childhood Depression Scale (CDS). The CDS was developed by Kovacs to determine the frequency of depressive symptoms. The scores over 19 were assessed for the presence of depressive disorder. EMF measurements in the classrooms were made with a Gaussmeter.

Results

The average age of the participants was $16.3 \pm 0.9$ years and $54.5 \%$ were female. The mean score of the students' depression scale was $17.3 \pm 6.5$. It was determined that $32.6 \%$ of the students were depressive symptoms. There was no significant relationship between the mean EMF level in the class and depression symptom frequency. According to the binary logistic regression model, the factors related to the depressive symptom frequency of the students are; being female $(\mathrm{OR}=1.61, p=0.031)$, having chronic disease $(\mathrm{OR}=2.31, p=0.004)$, poor school performance $(\mathrm{OR}=5.54, p=0.007)$, poor family relationship $(\mathrm{OR}=6.64$, $p=0.001)$ and the student's self-rated health status was low $(\mathrm{OR}=0.86, \mathrm{p} 0.001)$.

\section{Conclusion}

Approximately one in each of the three students had depressive symptoms. It is thought that to prevent depressive symptoms in the adolescent group, women should be evaluated as the primary risk groups and those with chronic diseases, school failure and family relations. Further research is needed to assess the association between depressive symptom frequency and EMF levels in class.

\section{5}

\section{Allergy \& Immunology}

Assessment of perinatal risk factors in children with allergic rhinitis in the Republic of Moldova

David Ala

${ }^{1}$ Department of Paediatrics, The State University of Medicine and Pharmacy "Nicolae Testemitanu", Moldova, Republic of

\section{Background}

In the last 10 years, the developed countries have been witnesses to a 1.52.0 fold increase in the prevalence of allergic rhinitis, which could be perceived as an epidemic. In the last five years, the Republic of Moldova has recorded an overall upward morbidity due to paediatric allergic rhinitis. Thus, in the reference period, the incidence rate went up by 2.39 times and the level of allergic rhinitis prevalence also increased by 2.11 times during the same period of time. It is well-acknowledged that perinatal risk factors are decisive factors in the development of allergic rhinitis in children at high risk, a statement supported by various authors.

\section{Objective}

To estimate perinatal risk factors in children with allergic rhinitis.

\section{Methods}

A questionnaire based selective descriptive study was conducted to estimate some perinatal risk factors in children with allergic rhinitis. The research cohort included 151 children with confirmed diagnosis of allergic rhinitis. The patients were interviewed based on the actual cases of the Departments of Allergology, Pulmonology and ENT from the Institute of Mother and Child.

\section{Results}

The percentage of children with an aggravated anamnesis on the mother's side (and/or her relatives) prevails $-43.7 \%$, followed by the brothers/ sisters' side $-31.7 \%$, and the father's side (and/or his relatives) $-24.6 \%$. We classified the perinatal risk factors in children with allergic rhinitis. Thus, the disregard of doctor's recommendations on hypoallergenic diet in pregnancy and during breastfeeding was ranked first $-49.0 \%$. The gestational age at birth (premature born babies and overdue babies) was ranked second $-42.0 \%$. The use of milk-based infant formulas was ranked third $-40.0 \%$. The early introduction of solids before 5 months was ranked fourth $-27.81 \%$, followed by the use of milk-based infant formulas across the first six months postpartum $-27 \%$.

\section{Conclusion}

The primary prophylaxis of paediatric allergic rhinitis is rooted in the outlining and ranking of the perinatal risk factors.

\section{3}

\section{Allergy \& Immunology}

\section{Kawasaki disease in two siblings of a Sub-Saharan family} Tom Balfroid ${ }^{l}$, Nancy Vitali ${ }^{l}$, Laurent $Y_{i}{ }^{l}$, Gilles Rondia ${ }^{2}$, Phu-Quoc L $\hat{e}^{l}$ ${ }^{T}$ Pediatric, Etterbeek-Ixelles hospital, Belgium

${ }^{2}$ Cardiology, Etterbeek-Ixelles hospital, Belgium

Kawasaki disease (KD) is an acute systemic vasculitis that predominantly affects children under 5 years old. Its major complication is the 
development of coronary arteries aneurysms. The underlying aetiology is still unknown but supposed to be a complex interplay of genetic susceptibility and/or an activation of the immune system triggered by an infection. We report hereby the case of one Cameroonian family with $2 \mathrm{KD}-$ affected siblings.

A girl of 8 months developed high fever since 9 days associated with cough, bilateral non-purulent conjunctivitis, cheilitis and impaired of general status. Laboratory features showed anemia, thrombocytosis, neutrophilia, high sedimentation rate and hyponatremia. Echocardiography revealed diffuse aneurysm formations of the coronary arteries. KD was suspected and the evolution is favourable under intravenous immunoglobulin (IVIG) and high-dose aspirin.

Her younger brother was diagnosed with KD 18 months later at 11 months old. He presented high fever since 3 days, with cervical lymphadenopathy and all mucocutaneous tissues alterations of the KD. IVIG and high-dose aspirin were given at the 7 th day from the initial fever with prompt defervescence. Unfortunately, coronary aneurysm was observed 20 days after the initial symptoms.

KD occurs worldwide with the highest incident in Japan and Korea. The incidence rates of KD were about 265 and 217 cases per 100,000 children under 5 years of age respectively. In contrast, in the United States, the estimated figure was lower at 19-25 per 100,000. In Japan, siblings' cases are well recognised. The risk is increased by 2 when the parents are affected with KD and by 10 in siblings of an index case. Furthermore, studies from Japan also suggest a higher risk of developing coronary artery lesions with families with multiple affected members.

Further genetic researches are needed to understand the physiopathology of KD. They could therefore lead to an improvement of its management.

\section{4}

\section{Allergy \& Immunology}

Fetal phagocytes loaded with Salmonella typhimurium or its flagellin trigger adaptive immunity in adult recipients

Jeng-Chang Chen ${ }^{1}$, Ming-Ling Kuo ${ }^{2}$

${ }^{T}$ Department of Surgery, Chang Gung Children's Hospital, College of Medicine, Chang Gung University,, Taiwan

${ }^{2}$ Department of Microbiology and Immunology, Graduate Institute of Biomedical Sciences, College of Medicine, Chang Gung University, Taiwan

\section{Background}

Our previous studies revealed that in utero exposure to ovalbumin could not elicit tolerance to ovalbumin but rather conferred immunity to ovalbumin. Such an event of in utero sensitization was caused by fetal phagocytes that attenuated proteolysis to sequester internalized ovalbumin and differentiated towards dendritic cells for delayed presentation.

\section{Objective}

We tried to manipulate fetal phagocytes as a strategy for cellular therapies against pathogens.

\section{Methods}

Fetal phagocytes were harvested from gestational day $14 \mathrm{FVB} / \mathrm{N}$ fetal liver and peritoneal cavity. Pooled fetal cells were pulsed with flagellin of Salmonella typhimurium (ST) or heat-killed ST overnight. Then, they were transferred intravenously into 4-6 week-old FVB/N mice. Foursix weeks later, recipients were examined for serum anti-flagellin or anti-LPS $\mathrm{IgG}_{1}$ levels by ELISA using mouse monoclonal anti-flagellin (FLIC-1, BioLegend) or anti-LPS $\operatorname{IgG}_{1}$ (1E6, Abcam) as the standard curve. Mice were also evaluated for the capacity of defending against lethal intraperitoneal challenge of live $S T$.

\section{Results}

We demonstrated that there were phagocytes within harvested fetal cells, capable of taking up bacterial bioparticles. Then, flagellin-loaded or heatkilled ST-loaded fetal cells were intravenously transferred into adult FVB/ $\mathrm{N}$ mice. Both antigen-loaded fetal cells caused significant secretion of anti-flagellin or anti-LPS $\operatorname{IgG}_{1}$, as compared with fetal cells only.
Following lethal challenge of live ST, ST-loaded but not flagellin-loaded fetal cells endowed the recipients with significantly better survivals than the controls of fetal cells only.

\section{Conclusion}

Fetal phagocytes loaded with $S T$ particles or its flagellin could trigger adaptive immunity in adult recipients to significantly secret antigenspecific $\operatorname{IgG}_{1}$. However, only $S T$-loaded fetal phagocytes conferred effective protection against lethal challenge of $S T$ in the generation of anti-LPS $\mathrm{IgG}_{1}$ in recipient mice. The ineffective protection against $S T$ in the presence of anti-flagellin $\mathrm{IgG}_{1}$ following the transfer of flagellin-loaded fetal phagocytes might be due to its low antibody titer generated.

\section{5}

\section{Allergy \& Immunology}

Complications of pregnancy in asthmatic womans and development allergy in their infants

Inna I. Evsyukoval

${ }^{T}$ D.O.Ott Research Institute of Obstetrics \& Gynecology, St-Petersburg, Russia

\section{Objective}

to determine the role pathology of pregnancy of the asthmatic womans in development of their infant's allergy. Methods. The analysis clinical state during first year of life 86 full term newborns, which mothers had bronchial asthma (BA): mild BA (63) - I group, moderate and severe BA(23) II group.. All infants received only the maternal milk to 5-6 month of life. Results. 1.No associations were observed with current symptoms of BA during pregnancy and the newborn's illness (IUGR), which was $38 \%$ in I and $39 \%$ in II groups. 2. The pathology of placenta were the main cause in development of allergy during first year of life. $36,5 \%$ women of I group had the chronic placental insufficient, $39,0 \%$ - pathologic immaturity of placenta and respectively $39,1 \%, 39,4 \%$ women of II group. 3 . Having pathologic immaturity of placenta with chronic placental insufficient were associated with more earlier (during the first month of life $21,4 \%$ infants) and more significantly higher risk of allergy after 6 month of life: $64,2 \%$ against $30,0 \%$ (p0,05) in infants which mothers hadn't such complications. Conclusion: The results suggest that pathology of the of placenta play the main role in alteration of functional activity antigen presenting cells of newborn's immune system and explain the association between number of future risk of atopic disease.

\section{9}

\section{Allergy \& Immunology}

\section{Component resolved diagnostics in severe allergy problems.}

Kaja Julge ${ }^{I}$, Tiina Rebane ${ }^{I}$

${ }^{T}$ Allergy Centre, Tartu University Hospital, Estonia

\section{Case history}

Current allergy tests define allergen-containing sources, not specific allergenic molecules. Molecular allergology improves clinical practice helping to differenciate true allergy from sensitisation, to predict the severity of the reaction and how long the allergy persist, to rationalise oral food challanges and make immunotherapy more effective.

Component resolved diagnostics was used for estimating sensitisation against foods and inhalant allergens in a child with severe allergy against several foods and inhalant allergens. Atopic dermatitis was diagnosed at 3 mo of age and due to very severe milk allergy only amino-acid based formula was possible to use. At 6 mo of age the level of IgE antibodies against casein, wheat, potato, cat was increased. At 2 yrs of age the child was sensitised against egg, rye, soy, mix of meets, nuts, tree pollens and at $3 \mathrm{yrs}$ the level of total IgE was over $5000 \mathrm{kU} / 1$. Using strict elimination diet and medication the symptoms of atopic dermatitis and asthma were almost under control. According component resolved diagnostics (Thermo Scientific, Immunocap ISAC) the child was multi-sensitised with IgE to species-specific and cross- 
reactive components. IgE to milk Bos $\mathrm{d} 8$ (casein)-7.2 ISU is associated with persistent milk allergy. IgE to egg Gal d1 (ovomucoid)-31 ISU is associated with persistent egg allergy. IgE to the storage proteins peanut Ara h1-1.7 ISU, hazelnut Cor a9-0.9 ISU, soybean Gly m6-0.6 ISU is associated with risk of severe allergic reactions. IgE to birch Bet v1-44 ISU may cross-react with pollen of related trees and plant food containing PR-10 proteins. IgE to cow Bos d6-3.2 ISU, cat Fel d2-1.9 ISU, dog Can f3-1.4 ISU explain crosssensitisation between furry animals and may cause allergic reactions after meat consumption or airway exposure to animal hair or dander.

In the presentation will be given overview of the course of the disease, diet, results of immunotherapy with milk.

\section{7}

\section{Allergy \& Immunology}

Sublingual imunotherapy: how satisfactory is it? ${ }_{\text {Maria Loureiro }}{ }^{2}$, Ana Azevedo ${ }^{I}$, Sandra Pereira ${ }^{1}$, Paulo Guimarães ${ }^{l}$, Arménia Oliveira

${ }^{1}$ Pediatrics, Centro Hospitalar Entre Douro e Vouga, Portugal

\section{Introduction}

Specific immunotherapy is the only treatment capable of impeding allergic disease progression and sensitization to new antigens.

\section{Methods}

We evaluated the degree of patient satisfaction with this line of therapy and its perceived efficacy through a questionnaire administered to outpatients undergoing specific sublingual immunotherapy (SSLIT) and followed in consultation at our department.

\section{Results}

Our sample consists of 119 answered questionnaires. Fifty-five percent of respondents were male $(n=66)$; median age 10 years-old; $53.8 \%$ had undergone SSLIT for two years or more at the time of answering; most frequent diagnoses: allergic rhinitis (AR) - $27.7 \%$; asthma - 25.2 \%; AR and asthma $37.8 \%$. Preventing disease progression was mentioned as the main reason for seeking therapy $(49.6 \%$ ) while the second most common was to lessen the number of flares $(30.3 \%)$, followed by wanting to be less reliant on medication $(20.2 \%)$. An overall improvement in night-time symptoms was reported (p0.01) as well as a lesser number of flares for both asthma and AR (p0.01). Patients noticed a reduced use of both SOS medication (p0.01) and basal medication. An improvement in tolerability for physical activity (p0.01) and perceived number of ER visits were also reported (p0.01). $97.5 \%$ of respondants refered improved overall symptoms and $92.4 \%$ would recommend the treatment to other patients. As the main positive aspects of this line of therapy respondents reported a overall better quality of life and less need of medication. The main downside reported was the price of treatment $(72.3 \%)$. Conclusions

Our results show an excellent degree of satisfaction with SSLIT and perceived symptomatic improvement, which demonstrates the value of this therapy in patients wellbeing and quality of life.

830

\section{Allergy \& Immunology}

Subcutaneous immunotherapy: the patients' point of view. Maria Loureiro $^{1}$, Sandra Pereira ${ }^{2}$, Ana Azevedo ${ }^{2}$, Arménia Oliveira ${ }^{2}$, Paulo Guimarães

${ }^{1}$ Pediatrics, Centro Hospitalar Entre Douro e Vouga, Portugal

${ }^{2}$ Pediatrics, Centro Hospitalar Entre Douro e Vouga, Portugal

\section{Introduction}

Asthma and allergic rhinitis (AR) interfere in the quality of life of patients. Specific subcutaneous immunotherapy (SSCIT) is a powerful therapeutic weapon which can prevent sensitization to new antigens and induce sustained clinical improvement.

\section{Objective}

To evaluate patients' satisfaction with SSCIT and its perceived efficacy.

\section{Methods}

We evaluated the degree of patient satisfaction through a questionnaire administered to outpatients undergoing SSCIT at our department.

Results

We had 94 answered questionnaires. $62.8 \%$ of respondents were male; median age: 14 years-old; $62.8 \%$ had undergone SSCIT for 2 years or more at the time of answering; polysensitization was present in $55.3 \%$ of patients; most frequent diagnoses: allergic rhinitis (AR) - $39.3 \%$; asthma $-21.3 \%$; AR and asthma $-39.4 \%$. Preventing disease progression was mentioned as the main reason for seeking therapy $(47.9 \%)$ while the second most common was to lessen the number of flares (33\%), followed by wanting to be less reliant on medication $(19.2 \%)$. An overall improvement in night-time symptoms was reported (p0.01) as well as a lesser number of flares for both asthma and AR (p0.01). Patients noticed a reduced use of both SOS medication (p0.01) and basal medication. An improvement in tolerability for physical activity $(\mathrm{p} 0.01)$ and perceived number of ER visits were also reported (p0.01). $97.9 \%$ of respondents would recommend the treatment to other patients. As the main positive aspect of this line of therapy respondents reported an overall better quality of life $(57.5 \%)$. The main downside reported was the price of treatment $(68.1 \%)$. Only $7.4 \%$ reported pain at the time of SSCIT administration. Conclusion

Our results are similar to what has already been described in the literature regarding SSCIT, symptomatic improvement and better quality of life. Immunotherapy should always be considered in the appropriate context, as it is the only treatment capable of stopping disease progression

411

\section{Allergy \& Immunology}

Eosinophilic Esophagitis - Experience of a Pediatric Allergology Unit Ana Neves ${ }^{l}$, Noemia Silval, Laura Azuraral, Ines Serras ${ }^{1}$, Joana Fermeiro $^{I}$, Ricardo Fernandes ${ }^{1}$

${ }^{1}$ Pediatric Department-Pediatric Allergology Unit, Centro Hospitalar Lisboa Norte - Hospital de Santa Maria, Portugal

\section{Background}

Eosinophilic esophagitis (EE) is a chronic, immunologically mediated inflammatory disease characterized by eosinophilic infiltration of the esophageal epithelium. There is significant associated morbidity, with a negative impact in the quality of life.

Our objectives were the immunoallergological characterization and the scientific review of EE, in children.

\section{Methods}

Retrospective descriptive study of the patients with the diagnosis of EE followed in the pediatric allergy consultation of a tertiary hospital during a one-year period.

\section{Results}

Eleven patients were included, all with a history of atopy. The most frequent presenting symptoms were dysphagia, abdominal pain, vomiting and food impaction. All patients performed skin prick tests (SPTs), which were positive for house dust mites in 9 cases, and for food allergens in 7 cases. Specific IgE was positive for the allergens detected in SPTs. Atopy patch tests and Immuno Solid-phase Allergen Chip (ISAC) were performed in two patients, showing that one was sensitized to allergens not previously detected in SPTs. The majority of patients are medicated with swallowed fluticasone. Those with food allergies are doing elimination diets for the identified allergens. During the follow-up period, all patients showed clinical improvement and three also revealed histological improvement.

\section{Conclusion}

We documented a greater predisposition for EE in males and a positive association with atopy. The presenting symptoms varied with age, with abdominal pain and vomiting being more frequent in young children, and dysphagia and food impaction in adolescents. The allergy assessment provided important clues to the etiology of the disease and helped in treatment management. 
328

\section{Allergy \& Immunology}

Overweight and obesity as a risk factor for severe wheezing in preschool children

Leticia Lambert $^{I}$, Leticia Lambert ${ }^{l}$, Luisa Savastano ${ }^{l}$, Stephanie Vargas $^{I}$, Etienne Duim ${ }^{2}$, Maria Celia Ciaccial ${ }^{1}$, Vera Rullo ${ }^{1}$

${ }^{1}$ Pediatric, Medicine University Fundação Lusiada, Brazil

${ }^{2}$ Public Health, Medicine University of São Paulo, São Paulo, Brazil

\section{Background}

Recently, overweigth/obesity were found to be a risk factor for asthma. Objective

The aim of the study was to investigate the relationship between obesity in early life and recurrent wheezing.

Methods

One-hundred and fifty pre-school children with recurrent wheezing were enrolled in this retrospective cohort study in Santos, Brazil. Children were followed in our pediatric allergy unit and the data of birth weigth, annual weigth and height, were reviewed from their electronic medical records from birth up to 5 years old, during the period from 2015 to 2017. International Body Mass Index (BMI) cut-offs were used to define: normal weight: $\mathrm{BMI} \leq 85 \mathrm{th}$; risk of overweight: $85 \mathrm{BMI} \leq 97 \mathrm{th}$; overweight: BMI 97th obesity: BMI 99.9. Recurrent wheezing was defined as three or more wheezing episodes in the past year. Severe wheezing was considered uncontrolled wheezing despite high doses of inhaled corticosteroids or that had indication to hospitalization. Infection of the upper or lower respiratory tract requiring antibiotics were recorded. The association between severe wheezing and risk factors studied was assessed by Poisson regression analysis at a $5 \%$ statistical significance.

\section{Results}

Adjusted analysis revealed that overweigth/obese children presented 2.23 of Incidence Rate Ratio to development of severe wheezing ( $p=0.002)$. On the other hand there was no significant association between low bith weigth or respiratory infection and severe wheezing.

\section{Conclusion}

Overweigth and obesity in early life were associated with severe wheezing in pre-school children.

\section{7}

\section{Critical/Emergency Care}

An Audit of Parents' Knowledge and Confidence in Managing Autistic Children following-CYGNET Training Programme at an Integrated Neurodevelopmental Service in South Eastern England Hani Ayyash ${ }^{1}$, Michael O. Ogundele ${ }^{2}$,Emma Weisblatt ${ }^{1,3}$, Lorraine Cuff ${ }^{I}$, Lorraine Azmi ${ }^{1}$, Tracey Bean ${ }^{l}$, Venkat Reddy ${ }^{l}$

${ }^{1}$ Cambridgeshire and Peterborough NHS Foundation Trust, University of Cambridge Health Teaching Trust, UK

${ }^{2}$ Community Paediatrics, Glenwood Health Centre, Glenrothes, NHS Fife, Scotland, UK

${ }^{3}$ Department of Psychology, Cambridge Laboratory for Research in Autism, University of Cambridge, UK

\section{Background}

Autism Spectrum Disorders (ASD) are neurodevelopmental social communication disorders, with common problems for families including sleep, anxiety, difficulty with change, sensory processing difficulties and disruptive behavior. The CYGNET programme was developed in 2003 by the UK-based charity Barnado as an information group for parents of children diagnosed with ASD. It is the primary post-diagnostic intervention offered in the Autism pathway in the Peterborough Neurodevelopmental Service.
Parents are routinely invited to attend the 6 session programme which aims to increase their understanding of ASD.

Aim

To evaluate the effectiveness of the CYGNET course in increasing parents' confidence in dealing with common situations relating to their child's ASD. Method

Data were collected retrospectively using an observational study design, using questionnaires completed by parents attending courses over a 12 month period.

Sixty-eight parents rated their confidence in dealing with 15 different common situations on a 6 point scale $(0=$ no confidence to $5=$ completely confident), at the start and end of the course. Paired t-test was used to compare total and item scores before and after the course. Fifteen questionnaires were excluded because of incomplete data leaving 53 participants.

Results

Self-rated the parents'/carers' confidence in dealing with the 15 situations was significantly greater following the programme completion in 51 (96\%) of the respondents (Figure 1). The mean increase in total score was 18.75 (95\% CI 16.11-21.40), $\mathrm{t}=-14.23, \mathrm{p} 0.0001$. The confidence ratings increased by over one point on average for each of the 15 situations (mean 4.0 vs 2.8 , mean difference $1.26 \pm 0.16,95 \%$ CI 1.16-1.35, $\mathrm{t}=29.94$, df 14, p 0.0001).

\section{Conclusion}

This study suggests that the CYGNET programme is effective in increasing the confidence of parents in dealing with difficulties arising when supporting young people with ASD. It would be useful to also evaluate longer-term effects of the CYGNET programme.

\section{3}

\section{Critical/Emergency Care}

Non-urgent visits of children with fever at the emergency care and observation department of the Children's Clinical University Hospital in Latvia

Anda Balode ${ }^{1}$, Dace Zavadska ${ }^{1,3}$, Dace Strazdina ${ }^{1,2}$

${ }^{T}$ Department of Pediatrics, Children's Clinical University Hospital,

Latvia

${ }^{2}$ Continuing Education Faculty, Riga Stradins university, Latvia

${ }^{3}$ Department of Pediatrics, Riga Stradins university, Latvia

\section{Background}

Increasing amount of non-urgent visits is one of the major problems in European countries at the emergency medical care of European countries, with fever being the most frequent reason for these visits. In Latvia the concept of non-urgent visits is not defined.

Methods

The specific study as a retrospective, descriptive study within the framework of European Union project's HORIZON 2020 PERFORM study MOFICHE took place from September 1, 2016 until May 31, 2017. A non-urgent visit within the study was considered to be every visit that met certain criteria based on source literature.

\section{Results}

These are preliminary results. Twenty percent of all the emergency care department visits (except for trauma patients) were visits by patients with fever. The most frequent triage priority for febrile patients at emergency department (ED) was non-urgent or "green" $-50 \%$ of all cases on average. Before going to ED a child's caretaker most often had complaints about fever that had lasted for less than 24 hours. Further diagnostics at ED were performed for $70 \%$ of all cases on average. Of all the febrile patient visits to $\mathrm{ED}$, below $20 \%$ were non-urgent visits according to the defined criteria. Within the non-urgent patient group (compared to the rest of the ED visit group) there was a statistically significant tendency of the parents/ caretakers more frequently being the ones who chose to go to $\mathrm{ED}$ (p0.001). 


\section{Conclusion}

Results from the study clearly indicate a necessity to perform a more in-depth and more comprehensive investigation and analysis of the non-urgent visits in order to assess and define non-urgent visits to the emergency care and observation department of Children's Clinical University Hospital and in accordance with health care in Latvia in general. Practical recommendations are necessary for parents on how to act if a child has fever.

\section{2}

\section{Critical/Emergency Care}

Patterns of Acute Poisoning in Children in Al Ain city (UAE)

Tarek I. El-Azzabi ${ }^{1}$, Jayalal Vasantha Nair ${ }^{l}$, Raied M Hasoon ${ }^{l}$, Rahat

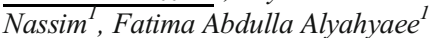

${ }^{I}$ Child Health Institute, Al Ain Hospital, United Arab Emirates

\section{Introduction}

Acute poisonings in children cause considerable morbidity and health resources use worldwide. It is a common presenting problem to paediatric emergency rooms (ER). This study was designed to describe the pattern of acute childhood poisoning in Al Ain.

\section{Method}

This is a retrospective study. It is involving children up to 13 year of age who attended our ER with acute poisoning between 1/6/2011 and 31/8/ 2014. We extracted data from the hospital patient electronic medical notes. We used electronic Word proforma and Excel to collect data. SPSS software was used for statistical analysis.

\section{Results}

Four hundred seventy-five children were recruited. The majority of the substances involved were pharmacological drugs $(40 \%)$ and household chemicals (29\%). In $78.5 \%$ cases, the culprit substance was not stored in the original container. Most children $(68 \%)$ were asymptomatic. No contact was made with a poisoning center in $92 \%$ of the cases.

For children with of acute non-caustic poisoning, charcoal was administered to 143 cases. $39.1 \%$ out of them received charcoal within one hour of ingestion of a substance. Gastric lavage was the second choice for decontamination (24\%). Approximately $47.6 \%$ of the children were admitted, mainly for observations (77\% stayed up to 24 hours). No reports of deaths from acute poisoning during the study. Diagnostic upper gastro-intestinal (GI) endoscopy was indicated in (11.9\%) of the caustic poisoning cases but none had the procedure.

\section{Conclusion and recommendations}

Acute poisoning substances pattern in $\mathrm{Al}$ Ain is not different from that in other countries of similar economic status. Pharmacological drugs and household chemicals represent the majority of the cases. This pattern has not changed for approximately two decades in comparison to previous data. We advocate education campaigns aiming to increase the general public awareness of the problem and to eradicate the habit of storing potential poisoning substances in secondary containers. There is a need to establish 24 hours poisoning center and review the criteria for diagnostic upper-GI in caustic-poisoning.

\section{2}

\section{Critical/Emergency Care}

Acute renal failure in oncologic pediatric patients ${ }_{\text {Bruno Espirito Santo de Araujo }}{ }^{1}$, Carolina Isidoro ${ }^{1}$, Fernanda Capela ${ }^{1}$,

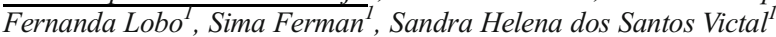
${ }^{1}$ CTI Pediatrico, Instituto Nacional de Cancer-INCA, Brazil

Between March 2014 and December 2016 eleven patients, (6 males, 5 females) with ages between less than 1 month (1), one children of 3 years old (yo) and 9 adolescents (10 to 18 yo); were admitted in the PICU with criteria of acute renal failure (ARF) and subsequent use of renal replacement therapy (RRT).
The causes of ARF were sepsis (7), tumoral lysis (1), bilateral nephrectomy (1), tumor compression (1) and chemotherapy (1)

The RRT was initiated less than 24 hours of admission in 6 patients and more than 24 hours (96 hours to 120 hours) in 5 patients. It was considered fluid overload when fluid intake-fluid output X-Weigh $(\mathrm{kg})$ X 100 were $10 \%$ in 24 hours.

All patients, but one underwent RRT by hemodialysis. The neonate used peritoneal dialysis. The types of neoplasia were: hemangioma (1), Leukemia (2), Linfoma (3), Osteosarcoma (1), rhabdomyosarcoma (1), Wills's tumor (1) and ovarian tumor (1).

The overall mortality rate was $18 \%$ (2) and one patient developed a chronic renal disease after discharge. The average of length of stay (LOS) in PICU was 27.5 days

Our data showed that $54 \%$ o the patients (6) had liquid overload just before the RRT and in that group, the mortality rate was significantly higher (33\%) when it was compared with the group with ARF (5) without liquid overload, which the mortality rate was zero. From those 6 patients, 2 underwent RRT in the first 24 hours of admission and 4 after 24 hours.

Although the number of patients was small, the results highlight the worse outcome in patients with liquid overload, which indicates the importance of early recognition and the prompt beginning of the RRT in acute renal failure in oncologic pediatrics patients.Further studies are necessary in order to validate the results.

\section{6}

\section{Critical/Emergency Care}

Developing a teaching faculty for the Emergency Paediatric Care Programme (EPCP) in Myanmar

Jay Halbert ${ }^{I}$, Jean Bowyer ${ }^{I}$, Michael Malley ${ }^{I}$, Pratusha Babu ${ }^{I}$, Marie Monaghan $^{1}$, Emma Roche ${ }^{l}$, San Oo ${ }^{l}$

${ }^{1}$ Global Child Health, Royal College of Paediatrics and Child Health, UK

\section{Purpose}

A skilled national teaching Faculty is central to the programme to improve clinical practice in district hospitals. The recruitment, training and activities of this group are described, with analysis of factors which enhance their impact.

\section{Methods}

During the EPCP courses outstanding participants are invited to attend a Generic Instructor Course (GIC) for further training in teaching skills before joining the Faculty as instructor candidates. Teaching is largely group-based, practical and highly interactive. Internationally experienced instructors mentor new instructors and assist the most experienced Myanmar doctors to be course-directors.

\section{Results}

Between June 2015 and August 2016 three GIC courses have successfully recruited 48 medical and nursing instructors. The new faculty have taught 37 times, contributing 3 course directors and half of the total activity. Twenty instructors have taught on at least two courses and are fully accredited. Nurses are diffident about teaching more senior staff, but have proved invaluable in ensuring smooth running and supporting other nurses. Junior doctors are generally less available to teach. Teaching is in Myanmar and in English, with vital explanations given by local instructors. They have also translated the final test and many of the materials. Feedback from instructors shows that they greatly appreciate the new teaching skills, the evidence based practice, teamwork and international contacts. They described a range of other EPCP-related activities on the wards, for MSc and medical students, and in outlying hospitals. Many have been instrumental in devising and implementing planned improvements, both through ongoing teaching and introducing new practices. One commented "I do love EPCP course and use its systematic approach not only in teaching but also in caring for the patients in daily ward works". 


\section{Conclusion}

The enhanced capacity of the faculty facilitates consistent teaching, improved senior buy-in, and better clinical practice. On them depends the long-term viability of this training course.

\section{1}

\section{Critical/Emergency Care}

Keeping Children Safe: Prevention and Management of Accidental Poisoning in Children

Laura Harrison $^{1,2}$, Maric Thorpe ${ }^{2}$

${ }^{T}$ Manchester Medical School, University of Manchester, UK

${ }^{2}$ Accident and Emergency Department, Nobles Hospital, Isle of Man

\section{Background}

Accidental poisoning in children is a common but preventable occurrence. In 2013-14, in the UK, almost 150,000 hospital admissions were due to poison exposure. Thankfully mortality is rare, however injury is still the leading cause of death in children, of which poisoning is the third most common mechanism. Objective

The aim of this project was to reduce incidence of accidental poisoning in children in an accident and emergency department and improve prehospital and immediate hospital management.

\section{Methods}

Firstly this project reviewed relevant literature on accidental poisoning in children, in particular focussing on epidemiology, looking at the age groups most commonly affected and the most common and most lethal substances ingested, and also the optimum pre-hospital management. This information was then used to devise a resource for parents, accessible to all, that advises on how to prevent accidental poisoning and what to do if this occurs. The relevance, usefulness and accessibility of this leaflet was audited, relevant adjustments made and the leaflet implemented. Suggestions were also made as to how doctors could aid in the prevention of accidental poisoning and how management could be improved both in the pre-hospital setting and in immediate hospital management

\section{Results}

The results showed that more could be done to prevent accidental poisoning in children and parents felt a lack of understanding about how to prevent accidental poisoning and what to do if it occurred. Our audit showed that our leaflet was able to provide parents with useful information and increase their understanding in these areas.

\section{Conclusion}

In conclusion, our study highlighted numerous areas in which accidental poisoning could be prevented or managed more effectively and a leaflet devised and suggestions made to rectify this. This could be implemented more widely across the UK and Europe and with the translation of the leaflet into other languages.

410

\section{Critical/Emergency Care}

Implementing Paediatric Sepsis Six: Our Experience Amy Lee ${ }^{I}$, Amitabh Gite ${ }^{I}$, Catherine Beadle

${ }^{T}$ Paediatrics, Lister Hospital, UK

\section{Background}

Sepsis campaigns in adults in the UK have been effective in improving early identification and management. There is limited data to assess the value of such campaigns in paediatrics. This project aims to assess compliance with the national standard and evaluate the effectiveness of a 'Raising Sepsis Awareness' (RSA) campaign in a district general hospital in the UK. Objective

A baseline audit was undertaken to assess compliance to national targets for sepsis screening and antibiotic administration over a 3 month period. National targets were set at $90 \%$ for screening compliance, and $68 \%$ for antibiotic administration within 60 minutes. A re-audit was undertaken to evaluate the effectiveness of the RSA campaign.

\section{Method}

Patients were identified using the hospital's electronic database and patient notes. Cases were reviewed to confirm the presence of sepsis triggers, appropriateness for screening and timing of antibiotics. Following the initial audit, we began a campaign which included teaching sessions for staff, posters displayed across the department and encouraging the use of sepsis toolkits informed by NICE guidelines.

\section{Results}

Thirty cases of sepsis were reviewed between July-September 2016, of which $100 \%$ (30/30) met diagnostic criteria. Screening compliance was $100 \%$ (30/30). In $43 \%$ (13/30) of cases, antibiotics were administered within 60 minutes. At 72 hours into admission, 25 cases still required antibiotics, with $100 \%(25 / 25)$ of patients being reviewed. In the re-audit between October-December 2016, 68 cases of sepsis were identified where $95.8 \%$ $(65 / 68)$ met diagnostic criteria. $100 \%(65 / 65)$ of cases were appropriately screened. Antibiotics were delivered within 60 minutes in $72.3 \%(47 / 65)$ of cases. All 59 patients requiring antibiotics at 72 hours were reviewed.

\section{Conclusion}

Our study showed a $29 \%$ improvement in compliance to national targets following the introduction of a RSA campaign. This study has demonstrated the value in educating staff and raising awareness of the paediatric sepsis six tool kit.

\section{4}

\section{Critical/Emergency Care}

Constrictive Pericarditis after Paediatric Cardiac Surgery: Case Report and Literature Review Hwee Ying Lim ${ }^{1}$, Ching Kit Chen ${ }^{2}$

${ }^{T}$ Department of Paediatric Medicine, KK Women and Children Hospital, Singapore

${ }^{2}$ Department of Paediatric Cardiology and Cardiothoracic Surgery, KK Women and Children Hospital, Singapore

\section{Objective}

Constrictive pericarditis (CP) is a rare occurrence post cardiac surgery in paediatric patients. Its cause is largely hypothetical and there is usually nothing significant found on review of the patients' peri-operative course. However, it is an important entity as it contributes significantly to morbidity and mortality. We aim to improve awareness of this rare complication for paediatricians involved in the post-operative care of patients with cardiac conditions.

\section{Case and Literature Review}

We present a case of a 10 year old child with a history of doubly committed juxta-arterial ventricular septal defect (VSD) who underwent patch closure via midline sternotomy. He presented 15 months later with reduced effort tolerance and was diagnosed to have $\mathrm{CP}$ and bilateral pleural effusions. $\mathrm{He}$ underwent a pericardiectomy and was discharged after 2 weeks.

$\mathrm{CP}$ is a well-known complication post cardiac surgery in adult literature. However, to our knowledge, there are only 6 cases of $\mathrm{CP}$ occurring as a complication post cardiac surgery in current paediatric literature, making our case the 7 th report. Of the 7 , there are 4 cases noted occurring post VSD closures, denoting a possible implication. Pericardiectomy remains the definitive form of therapy for post-operative $\mathrm{CP}$ and majority of paediatric cases do well post-pericardiectomy.

\section{Conclusion}

$\mathrm{CP}$ is a rare but important complication post invasive heart surgery in children. Time of occurrence is variable ranging from months to years post-surgery. Presentation may be non-specific and clinicians need to be aware of the condition to have a high index of suspicion. Majority of paediatric cases do well post-pericardiectomy which is the treatment of choice in this condition. This is in contrast with reports from adult literature and may be attributed to comorbidities and nature of cardiac surgery in the adult population. 
Pooling data from current and future literature would be beneficial for further research.

649

\section{Critical/Emergency Care}

"On-Call" Tasks in the Paediatric Cardiology Unit - A Quality Improvement Project

Siobhan McCormack ${ }^{1}$, Catherine Breen ${ }^{I}$, Terence Prendiville ${ }^{1}$

${ }^{T}$ Department of Paediatric Cardiology, Our Lady's Children's Hospital, Crumlin, Dublin, Ireland

\section{BACKGROUND}

The national cardiology inpatient unit cares for some of the most complex and acutely unwell children in Ireland. Such patients have frequent alterations in clinical state necessitating frequent review and often have complex care plans and early warning score amendments in place. Oftentimes medical input to these patients on-call cannot be anticipated, however it was hypothesised that much of the task burden for house officers could be dealt with during, and is more appropriate to, routine hours and familiar team input to achieve improved quality patient care. Reducing time spent on routine tasks on-call also increases time available for direct patient care. AIMS

To examine the variation, content and quantity of tasks requested of the on-call house officer on the cardiology unit and to seek to improve the number of tasks which would be better served, and could be anticipated by medical input during routine hours.

\section{METHODS}

The house officer on-call tasks were recorded on a specifically designed project sheet from a sample period in March and April 2017 and were analysed using Excel. The results were presented to the cardiology team in chart format and discussed as a continuous measure.

RESULTS

The most frequent tasks involved charting and altering medications, in particular analgesia and amending early warning scores. A smaller proportion of these tasks necessitated medical reviews. As the study period progressed the number of routine tasks declined as these areas were brought to the attention to the primary team. Medical reviews remained stable throughout the period.

\section{CONCLUSION}

Awareness of the on-call tasks by the responsible team lead to an improvement in the number of avoidable routine tasks passing forward to-on call doctors while unanticipated review remained appropriately stable. This has favourable impact on time management and improving quality of patient care.

723

\section{Critical/Emergency Care}

ALTE to BRUE: Assessing the Implications of the American Academy of Paediatrics First Clinical Practice Guideline for the Management of Apparent Life Threatening Events Leona Nertney ${ }^{1}$, Paul McNally ${ }^{l}$, Ikechukwu Okafor ${ }^{2}$

${ }^{T}$ Department of Paediatrics, Royal College of Surgeons in Ireland, Ireland

${ }^{2}$ Emergency Department, Temple Street Children's University Hospital, Ireland

\section{Background}

Apparent Life Threatening Events (ALTE) can prove challenging due to lack of consensus on management. In 2016, the American Academy of Paediatrics produced its first clinical practice guideline to address this. These propose a nomenclature change, replacing $A L T E$ with a new term Brief Resolved Unexplained Event (BRUE), criteria to risk stratify infants, and management of lower risk infants. It is unclear what proportion of infants will be reclassified as low-risk and the potential health service implications of this.

\section{Objective}

To assess the potential health service utilisation implications of AAP Guidelines specific to Apparent Life Threatening Events.

\section{Methods}

Retrospective review of ALTE presentations (January-December 2016) to a large Paediatric Emergency Department. Cases were reclassified as low-risk BRUE if they met all the following criteria: age60 days; gestational age $32 / 40$ or post-conceptual age 45 weeks; 1st event; no CPR required by trained provider; no significant past medical history; no concerning historical features or physical examination findings; event lasting 1 minute duration.

\section{Results}

Data from 39 patients diagnosed with an ALTE were analysed (mean age $8.1+/-9.9 \mathrm{wks} ; 20(51.3 \%)$ female). Of these $9(23.1 \%)$ were reclassified as a low-risk event (mean age $17.3+/-10.7 \mathrm{wks} ; 7$ (77.7 \%) female). This group had a median length of stay of 2 days, with a total of 20 infant bed days used (19.1\% of total ALTE cohort). 54 (18.3\%) of total ALTErelated investigations were conducted on this group.

\section{Discussion/Conclusion}

This is the first study to assess the impact of introducing AAP Guidelines specific to ALTE presentations. 1 in 4 presenting with an ALTE can be reclassified as a low-risk BRUE. Benefits to both the patient and hospital are evident, including reduction of unwarranted invasive investigations, unnecessary radiation exposure plus avoidance of hospital admissions. The new guidelines offer a simplified means to risk stratify and manage these patients.

255

\section{Critical/Emergency Care}

Evaluation of Cases With Forensic Qualifications Applying to The Emergency Service In A Hospital

Seva Oner ${ }^{1}$, Leyla Uces Harmanogullari ${ }^{1}$, Gulcin Yapici ${ }^{1}$, Sevcan Ozel ${ }^{2}$ ${ }^{T}$ Department of Public Health, Mersin University Faculty of Medicine, Turkey

${ }^{2}$ Health Sciences Institute, Mersin University Faculty of Medicine, Turkey

\section{Background}

It is emphasized that children's rights must be protected against all kinds of physical or mental assault, violence, abuse, neglect or abuse. However, one in every four children is exposed to neglect/abuse at least once in their lifetime. Objective

In this study, It was aimed to evaluate the cases with forensic qualifications who applied to the pediatric emergency department in a hospital.

\section{Methods}

The sample group of this descriptive study constitutes cases with forensic qualifications of Mersin Obstetrics, Gynecology and Pediatrics Hospital, Pediatric Emergency Service that were applied in January-April-July-October 2016. Five thousand six hundred seventy-five of them were cases with forensic qualifications. The data were collected between 01.11.2016-14.11.2016. The data of the study were obtained by examining the patient records at the indicated 4 months. Descriptive statistics (mean, percent) were used in the analysis of the data.

\section{Results}

$5675(6.54 \%)$ of the 86752 applications made to the pediatric emergency department in the 4 months of 2016 constituted cases with forensic qualifications. The mean age of cases was $6.4 \pm 4.8$ years $(\min =0, \max =18) .60 .3 \%$ of the cases were male. $6.2 \%$ of the cases were hospitalized. $8.5 \%$ of cases were reported as judicial cases. $75.8 \%$ of the cases were injured/falls, $6.7 \%$ poisoning, $5.3 \%$ burns, $3.8 \%$ foreign body ingestion, $2.9 \%$ animal bite, $2.8 \%$ cutter or penetrative tool injury, $1.4 \%$ traffic accidents, $0.4 \%$ electric shock, $0.4 \%$ neglect/exploitation, $0.3 \%$ strike, $0.1 \%$ water drowning and $0.1 \%$ suspicious death.

\section{Conclusion}

Approximately four-thirds of the cases were injuries and falls, followed by poisonings. In childhood, injuries and poisonings 
are important in terms of preventable health problems and being at the forefront of the causes of disability. All cases with forensic qualifications should be detected by the physician, forensic report should be prepared and necessary treatment and rehabilitation services should be presented to the children.

813

\section{Critical/Emergency Care}

Acute intoxication in Pediatric Emergency Department: a 12 months survey

Francesca Rech Morassutti ${ }^{1}$, Carlo De Pieri ${ }^{l}$, Anna Pusiol ${ }^{l}$, Paola Cogo ${ }^{l}$ ${ }^{T}$ Department of Clinical and Experimental Medical Science, University Hospital of Udine, Pediatric Clinic, Italy

\section{Background}

Acute intoxication (AI) is a major risk of morbidity and mortality among pediatric population, especially in toddlers. Small literature on the modality of admission to the emergency department (ED) following AI and outcome management is available.

\section{Objective}

To assess the epidemiology of first evaluation following AI in a third level pediatric ED

Methods

We retrospectively reviewed medical record of all children aged 0-16 years admitted to ED for AI. Patients evaluated from April 1, 2016 to March 31, 2017 to the pediatric ED of University Hospital of Udine were enrolled. Admissions for foreign body ingestion were excluded. For each patient we collected: gender, age, date and time of admission, access modality, triage code, discharge code and eventual hospital admission, substance category.

\section{Results}

Patients evaluated for AI were 73 among a total of 14795 ED admission. Male were $52.1 \%$. Median age was 21 months (IQR 14-57). Of the evaluated children $65.7 \%$ were

Conclusions

Ours results reflect literature data, adding lacking information especially about our region. We found of interest that despite AI scares healthcare professionals, no red code were assigned, neither in the two cases that need ICU admission.

\section{0}

\section{Critical/Emergency Care}

Child With Fever At The Emergency Department And Observation Unit Of The Children's Clinical University Hospital: Clinical Characteristics And Treatment Tactics

Dace Strazdina ${ }^{1,2}$, Dace Zavadska ${ }^{1,2}$, Anda Balode ${ }^{l}$

${ }^{T}$ Department of Pediatric, Children Clinical University Hospital, Latvia

${ }^{2}$ Department of Pediatric, Riga Stradins University, Latvia

\section{Introduction}

Management of a child with fever is one of the most frequent and most important health care problems in the world. Majority of increased body temperature in children are related to a self limiting virus caused acute infection and indeed rarely the cause of fever is related to a serious bacterial infection. Fear of increased body temperature in a child is the most frequent reason for parents to visit hospital emergency units.

Methods

Retrospective, descriptive study within the framework of European Union project's HORIZON 2020 PERFORM study MOFICHE. Study included all patients who visited Children clinical university hospital (CCUH) in Riga Emergency Department (ED) with complaints of fever $\left(38^{\circ} \mathrm{C}\right.$ and over) at the time period from September 1 till December 31, 2016. Data was selected from the ED paper and electronic case files by using a specially created MOFICHE case record form.

\section{Results}

Preliminary data - ED was visited by 2720 patients with complaints of febrile body temperature during the study period. Most frequent cause of fever was upper respiratory tract infection (otitis, tonsillitis, pharyngitis, rhinitis) $-25 \%$. Based on complaints, objective findings, and laboratory results $5 \%$ of the fever patients had proven bacterial infection. Following the complaint of fever, the next more frequent were: rhinitis $20 \%$, cough $25 \%$, vomiting $15 \%$, diarrhoea $10 \%$. Antibacterial therapy was prescribed for $30 \%$ of all febrile children who visited ED during the study, in which bacterial infection was proven for $10 \%$ of these children.

\section{Conclusions}

The average CCUH ED patient within the mentioned time frame was a child of up to 4 years of age who most frequently had a viral upper respiratory tract infection with possible self limiting course of disease; treatment and health care most frequently corresponds with that of a primary care patient.

\section{9}

\section{Critical/Emergency Care}

Beware of the booby trap: behavioral and psychiatric symptoms may mask serious endogenous pathology in children. Viola Van Gorp ${ }^{1}$, Hendrik Discart ${ }^{3}$, Gerlant van Berlaer ${ }^{2}$, Ives Hubloue ${ }^{2}$

${ }^{T}$ Pediatric Critical Care Department, UZ Brussel, Belgium

${ }^{2}$ Department of Emergency Medicine, UZ Brussel, Belgium

${ }^{3}$ Department of Emergency Medicine, Jessa Ziekenhuis, Belgium

\section{Background}

Pediatric patients present to the Emergency Department (ED) with a wide variety of pathology. In case of altered behavior in children, fast recognition, diagnosis and treatment are indicated. In pediatric patients presenting with "abnormal behavior", it may be very difficult to differentiate between real psychiatric disorders - a growing problem in Western countries - or abnormal behavior masking other underlying organic pathology. Methods

Cases of pediatric ED patients, initially diagnosed with mood and behavioral changes, but whom developed severe organic pathology during admission, were reviewed regarding initial symptoms, final diagnosis, time from initial to final diagnosis, length of stay (LOS) in the hospital and days in the PICU, and outcome.

\section{Results}

Nine patients were included. The median age was 11 years old (range 1 to 18 years-old, 5 were female and 4 were male. Although initial symptoms rather resembled psychological-behavioral disorders, the final diagnoses were Anti-N-methyl-D-aspartate receptor encephalitis (ANMDAR), 1 case due to Mycoplasma pneumoniae and 1 case due to toxoplasmosis, two cases with acute disseminated encephalomyelitis (ADEM), Guillain Barré syndrome, epileptic state, partial convulsions, confusion due to $\mathrm{AKI}$ and a cerebral tumor. Median hospital LOS was 12 days, and median ICU stay was 7 days. Median time to final diagnosis was 2 days. All patients' neurological outcome improved over time.

\section{Conclusion}

Pediatric patients presenting to the ED with behavioral changes or psychiatric symptoms, may suffer from more serious organic pathology than initially suspected.

Physicians need to pay attention to this "booby trap", in order to prevent long-term neurologic damage, an increasing risk of debilitating complications, or even death.

\section{7}

\section{Critical/Emergency Care}

Acute peritonitis due to uncommon bacteria Nicola Zampieri ${ }^{l}$, Marta Peretti ${ }^{l}$, Federica Bianchi ${ }^{l}$, Simone Patanè ${ }^{I}$, Virginia Murri $^{2}$, Francesco Camoglio ${ }^{1}$ 
${ }^{1}$ Pediatric Surgical Unit, Azienda Ospedaliera Universitaria Integrata, Italy

${ }^{2}$ Department of Pediatrics, Azienda Ospedaliera Universitaria Intergata, Italy

\section{INTRODUCTION}

Acute appendicitis is the most comment urgency in pediatric population. some cases has peritonitis with complicated appendicitis; the aim of this study is to report our experience with severe peritonitis caused by strange bacteria

\section{MATERIALS AND METHODS}

We retrospectively analyzed cases treated for peritonitis related to acute appendicitis, between December 2010 and December 2016 aged between 0 and 14 years; all medical charts were reviewed and analyzed.

\section{RESULTS}

Among 138 patients with peritonitis 8 cases were studied; during hospital stay we were informed by the pathology service about the bacteria extracted into the appendix and into the peritoneal fluid. One case was diagnosed as having Giardia Lamlia, 4 cases were peritonitis due to enterobius vermicularis, one case was Yersinia enterocolitica and two cases were non typhoidal salmonella. Four patients developed intraabdominal abscess after surgery that were treated with surgical drainage in two cases and conservatively in the other.

\section{CONCLUSIONS}

Even if acute appendicitis with peritonitis is not a rare association, there are some cases that require different treatment; rare pathogens could be the cause of these peritonitis and it is better to have a complete fluid analysis are histological evaluation in order to have the best treatment options

169

\section{Critical/Emergency Care}

Decision Tree for Admission of Infants Under 6 months of Age Presenting with Non-specific Complaints

Michael Zhang $^{1,3}$, Marie-Louise Ratican ${ }^{2,3}$, Nicholas Marriage ${ }^{3}$, Valerie Astle ${ }^{3}$, Jonathan Ash ${ }^{3}$, Haddijatou Hughes ${ }^{3}$

${ }^{1}$ School of Medicine and Public Health, University of Newcastle, Australia

${ }^{2}$ Department of Medicine, Queens University, UK

${ }^{3}$ Emergency Department, John Hunter Hospital, Australia

\section{BACKGROUND}

Young infants are brought to the Emergency Department (ED) with a variety of complaints. Some of these complaints can be non-specific to the carers and the clinicians attending these infants. They are assessed thoroughly after arrival and sometimes have to undergo a variety of investigations for serious illnesses. When the serious diseases are confirmed or cannot be excluded in ED, the infants need to be admitted to the hospital for further management. This study was proposed to examine a prediction model for the young infants with nonspecific complaints.

\section{OBJECTIVE}

This research project aimed to derive a prediction model for admission from the pertaining features of the young infants presenting to the ED.

\section{METHODOLOGY}

This was a retrospective observational study examining the clinical features and the disposition outcomes of young infants under 6 months presenting to a mixed ED in the 2013-14 period. The infants deemed to have non-specific complaints or diagnoses by the clinicians in the Emergency Department were included. With recursive partitioning statistical technique, clinical data of eligible patients extracted from electronic medical record were used to construct a decision tree models of risks for admission.

\section{RESULTS}

The admission rate for these 968 infants studied was $33.6 \%(95 \% \mathrm{CI}$ $30.59-36.55 \%)$. Some risks factors associated with higher chance of admission were identified through statistical modelling. A decision tree incorporating the most important risk factors was constructed, that has a sensitivity of $85.96 \%$, specificity $97.99 \%$ and a negative prediction value $93.01 \%$. Its misclassification rate was only $6.15 \%$.

\section{CONCLUSION}

Prediction models with excellent accuracy can be built on the features of young infants presenting with non-specific complaints. They could be used to guide decision making and as a bench marking measure for institutional comparisons.

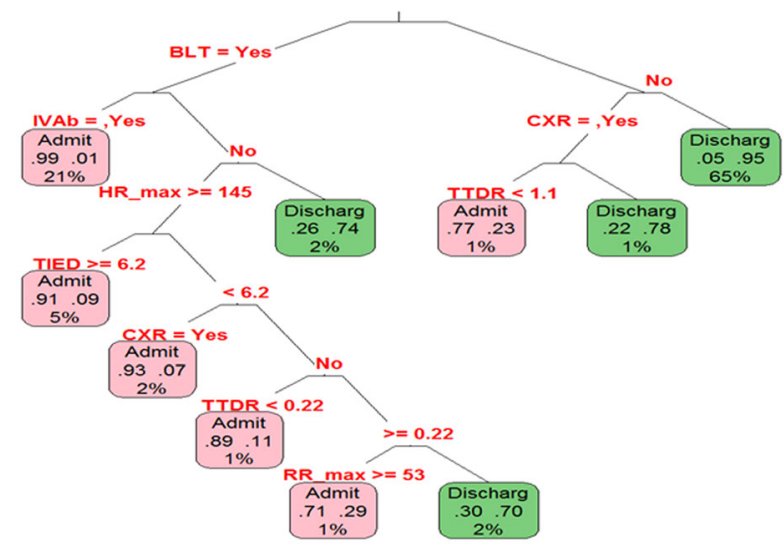

Tracking the path from the first node to the terminal mode, following the left branch if the condition at a node is satisfied The majority class was used to label the terminal nodes. The proportions below the major class represented the probahility of eac $h$ class. Percentage of the patients was shown at the bottorn of the terminal boxes. BIT, Blood Test Done. CXR, Chest Xray- IVAb, Intravenous Artibiotics. HR_max, Maximum heart rate. TTDR, Time first seen by doctor. TIED, Time staying in ED. RR_max, maximum respiratory rate

291

Endocrine \& Diabetes

Dental health in children with diabetes mellitus

Bibigul Abeuova ${ }^{I}$, Svetlana Tuleutaeyva ${ }^{I}$, Zhadyra Ashirbekova ${ }^{I}$

${ }^{T}$ Department of Children's Diseases, Karaganda State Medical University, Kazakhstan

There are 225,735 patients with diabetes mellitus (DM) in Kazakhstan, children under 18 years of age with type 1 diabetes - 1,801 of them. Often oral diseases are noted in children with type 1 diabetes, which according to the literature are observed in $87.9 \%$ of patients.

\section{OBJECTIVE}

To study the prevalence and intensity of caries and periodontal diseases in children with type 1 diabetes

\section{MATERIALS AND METHODS}

Sixty children with type 1 diabetes undergoing in-patient treatment at the Regional Children's Clinical Hospital in Karaganda were examined. Patients were divided into three groups depending on the level of glucose: in children of group 1 the blood glucose level was less than $10 \mathrm{mmol} / \mathrm{L}$, in group 2 - from $10 \mathrm{mmol} / \mathrm{L}$ to $20 \mathrm{mmol} / \mathrm{L}$, in 3 Group - more than $20 \mathrm{mmol} / \mathrm{L}$. Indices of hygiene of the oral cavity, intensity and prevalence of caries and periodontal diseases were defined.

\section{RESULTS}

In 15 children with DM 1, a compensated form of caries was observed, subcompensated in 36 children decompensated in 9 children.

Pathological changes in periodontal tissue are noted in all cases. It was revealed: $100 \%$ bleeding gums, $100 \%$ presence of supra- and subgingival tartar, $87.5 \%$ of cases of inflammation of the gingival papilla, $80 \%$ of the marginal and $55 \%$ of the alveolar gum.

\section{CONCLUSIONS}

- The prevalence of caries in children with type 1 diabetes was $70 \%$ and was characterized as an average. The intensity of caries in children with type 1 diabetes by the DMF index was $\mathrm{M}=8.7 \mathrm{SD}=2.2$.

- The prevalence of periodontal diseases in children with diabetes is characterized as high and is $100 \%$. The intensity of periodontal disease is estimated as an average and is $\mathrm{M}=2.28, \mathrm{SD}=0.47$ 
306

\section{Endocrine \& Diabetes}

Endocrine disrupters and hyperestrogenism Ruxandra Simona Bacanu ${ }^{1}$, Mauricio Moreno López ${ }^{1}$

${ }^{T}$ Pediatrics, Tomelloso General Hospital, Spain

\section{Background}

Bloody nipple discharge rarely occurs in infants, but represents a cause of stress for the parents. Sometimes could be produced by an increase estrogen levels from an external source, represented by an endocrine disruptors (cosmetic products with estrogen, medication, food).

\section{Aim of paper}

To demonstrate that the bloody nipple discharge in childhood is frecuently benign and it's related with endocrine disrupters.

\section{Matherial and method}

We present a 15 months-old girl with bloody nipple discharge without a history of medication or trauma. Physical examination revealed a healthy child with normal findings. At the left breast the skin had a normal aspect, with no injuries or palpable masses. Pressure on the areolar area didn't bring us any new. We realized a breast ultrasound and blood analizes. All was normal, but a high level of estradiol was found. We have considered there is a disruptive endocrine factor, thinking in the estrogens from chicken breast (presented every day in her diet). So we have advised parents to remove the chicken breast from the girl's diet. After two months, the estrogen level was normal and the girl has no more bleeding, as well as one year later.

\section{Discussion}

As we comment, there were no history of medication or application of cosmetic products at breast region. We considered that the hyperestrogenism could be caused by the contamination by dietary intake of chicken breast. Chicken is often injected with estrogen for obtaining much breast chicken; when we eat it, this hormons will be transferred to our body. The children are more vulnerable at these hormons and could develop various clinical manifestations.

\section{Conclusion}

In our patient we found an increased estrogen level from diet. Therefore the level of estrogen as well as the hypertrophy of glandular tissue regressed within 2 months with the change of the diet.

\section{9}

\section{Endocrine \& Diabetes}

A novel compound heterozygous mutation in a Chinese boy with Thiamine-responsive megaloblastic anemia

Qing Cheng ${ }^{l}$, Xin $\mathrm{Li}^{l}{ }^{l}$, Qun $\mathrm{Li}^{l}$, Yu Ding ${ }^{l}$, Juan $\mathrm{Li}^{l}$, Yao Chen ${ }^{l}$, Guoying Chang $^{I}$, Jian Wang ${ }^{2}$, Xiumin Wang ${ }^{1}$, Yijin Gao ${ }^{3}$

${ }^{1}$ Department of Endocrinology and Metabolism, Shanghai Children's Medical Center, Shanghai Jiaotong University School of Medicine, China ${ }^{2}$ Department of Medical Genetics and Molecular Diagnostic Laboratory, Shanghai Children's Medical Center, Shanghai Jiaotong University School of Medicine, China

${ }^{3}$ Department of haematology and haematological oncology, Shanghai Children's Medical Center, Shanghai Jiaotong University School of Medicine, China

\section{Background}

Thiamine-responsive megaloblastic anemia (TRMA), a rare autosomal recessive inherited disease, is characterized by the clinical traid of diabetes mellitus, megalobastic anemia, and sensorineural deafness. Some of these symptoms can be relieved by therapeutic dose of thiamine. To date, only 100 cases have been reported in the world and 4 in China. We aim to report a novel compound heterozygous mutation in a 6 years old Chinese boy who presented with typical clinical features of TRMA.

\section{Methods}

We collected the boy's clinical presents, and past history. Then we did some examinations for him, such as CBC, serum glucose, serum insulin, serum iron and so on; Besides, the boy was evaluated by the targeted next generation sequencing using his peripheral blood genomic DNA, and the relevant mutation was verified with Sanger sequencing.

Results

The boy presented diabetes mellitus, anemia and deafness. Meanwhile, he also presented thrombocytopenia, leukopenia, horizontal nystagmus, thrombocytopenia, leukopenia, ventricular premature beat, hepatomegaly, and short stature. DNA sequencing revealed a novel compound heterozygous mutation in SLC19A2: (1) gene duplication c.405dupA, p.Ala136Serfs*3 (heterozygous); (2) nucleotide deletion c.903delG p.Trp301Cysfs*13 (heterozygous). The boy was diagnosed with typical TRMA. After thiamine therapy, the diabetes and anemia of him was improved.

\section{Conclusions}

This is the first report of SLC19A2 gene heterozygous mutation in Chinese patient, our finding expands the mutant spectrum of SLC19A2 gene and adds new understanding of the phenotype.

Key words: Thiamine-responsive megaloblastic anemia; SLC19A2 gene; novel mutation; diabetes; megaloblastic; deafness; targeted next generation sequencing

\section{6}

\section{Endocrine \& Diabetes}

Bisphenol A (BPA) Exposure and Risk of Idiopathic Central Precocious Puberty (ICPP) among School-aged Girls in Shanghai, China

Yao Chen ${ }^{1,2}$, Yingcan Wang ${ }^{1,3}$, Zhijun Zhou ${ }^{4}$, Guodong Ding ${ }^{1,5}$, Ying Tian $^{1,6}$, Xiumin Wang ${ }^{2}$, Lixiao Shen ${ }^{1,3}$, Hong Huang ${ }^{1,6}$

${ }^{I} M O E$ and Shanghai Key Laboratory of Children's Environmental Health, Xinhua Hospital, School of Medicine, Shanghai Jiao Tong University, China

${ }^{2}$ Department of Endocrinology and Genetic Diseases, Shanghai Children's Medical Center, School of medicine, Shanghai Jiao Tong University, China

${ }^{3}$ Department of Children Health Care, Xin Hua Hospital, School of medicine, Shanghai Jiao Tong University, China

${ }^{4}$ Department of Occupational Health, School of Public Health, Fudan University, China, China

${ }^{5}$ Department of Pediatrics, Shanghai East Hospital, School of Medicine, Tong Ji University, China

${ }^{6}$ Department of Environmental Health, School of Public Health, School of Medicine, Shanghai Jiao Tong University, China

\section{Background}

It is supposed that exposure to Bisphenol A (BPA) may increase the risk of precocious puberty (PP) in girls. However, few studies have investigated the relationship between urinary BPA levels and idiopathic central precocious puberty (ICPP) in school-aged girls in China.

\section{Objective}

We investigated total BPA concentration in subjects' urine and examined the correlation with ICPP in school-age girls in Shanghai.

\section{Methods}

We conducted a 1:1 matched case-control study of ICPP among schoolaged girls in Shanghai. The study included 136 girls diagnosed with ICPP aged $6-9$ years and 136 healthy girls matched for age. Total urinary BPA concentrations were determined for all subjects. Medical information of ICPP girls was recorded.

Results

BPA was detected in $83.8 \%$ of urine samples in ICPP group, which was significantly higher than the control group with a percentage of $58.8 \%$ $\left(\mathrm{X}^{2}=16.02, p=0.000\right)$. Median levels of urinary adjust-BPA in ICPP group and control group were 6.35 and $1.17 \mu \mathrm{g} / \mathrm{g}$ creatinine, respectively $(Z=-6.345, p=0.000)$. After adjustment for confounders, compared with those in the lower quartiles of adjust-BPA, the higher quartiles were associated with a 1.47-fold increased risk of ICPP [Odds ratio (OR) 
$=1.47,95 \%$ confidence interval $(\mathrm{CI}): 1.22-1.77, p=0.000]$. In the ICPP group, weak negative correlations were presented between urinary adjustBPA levels and basal FSH levels $(r=-0.236, p=0.006)$ and peak FSH levels $(\mathrm{r}=-172, p=0.045)$.

\section{Conclusion}

The present findings suggest that BPA exposure may be associated with an elevated risk of ICPP in girls. The mechanism may be related to the relatively lower levels of FSH.

719

\section{Endocrine \& Diabetes}

Small gestational age - implications of chronic autoimmune thyroiditis in pregnancy

Eduard Circo ${ }^{1}$, Ibadula Seila ${ }^{l}$, Scrinic Olesea ${ }^{l}$

${ }^{1}$ Endocrine, Clinical Emergency County Hospital of Constanta

- "Sf.Apostol Andrei", Romania

\section{Objective}

Implication of thyroid autoimmune disease of pregnant women on some somatic parameters in newborns.

\section{Material and Method}

One thousand eight hundred fifty-nine term newborns were evaluated. Mothers were investigated of thyroid hormonal function (TSH, FT4) and thyroid autoantibodies (ATPO, antithyroglobulin antibodies) were determined. The results divided the mothers with chronic autoimmune thyroiditis into two groups: group 1 - chronic autoimmune thyroiditis present with normal thyroid function; group 2- chronic autoimmune thyroiditis with hypothyroidism.

A questionnaire for iodine supplements taken during pregnancy was completed. For newborns were assessed: weight at birth, Apgar score, and gestational age. A control group of 784 newborns from mothers with no thyroid problems was studied comparatively.

\section{Results}

Newborns from mothers with chronic autoimmune thyroiditis had a lower birth weight compared to children of the same gestational age with mothers without thyroid pathology. The incidence of newborns with SGA was statistically significant in group $2(3.5 \%)$ than group $1(3.3 \%)$ compared to the control group (2.5\%) (p 0.05). Consumption of iodinated supplements was $-31.6 \%$ in group 1 and $-50 \%$ in group 2 respectively.

\section{Conclusions}

Chronic autoimmune thyroiditis can be considered a risk factor for SGA. The impact of maternal hypothyroidism in pregnancy increases this risk. Supplementation with iodine in pregnant women with chronic autoimmune thyroiditis simultaneously requires a dynamic evaluation of thyroid hormonal profile.

\section{8}

\section{Endocrine \& Diabetes}

Pituitary Adenoma Coexisting with Neurofibromatosis Type 1 in Childhood. A Case Report

Smaranda Diaconescu ${ }^{1,3}$, Claudia Olaru ${ }^{2,3}$, Nicoleta Gimiga ${ }^{2,3}$, Cristina Raluca Stanca ${ }^{3}$, Marin Burlea ${ }^{2,3}$, Ileana Ioniuc ${ }^{2,3}$

${ }^{1}$ Medicine, "Titu Maiorescu" University, Romania

${ }^{2}$ Medicine, "Gr.T.Popa" University, Romania

${ }^{3}$ Pediatrics, "St. Mary" Clinical Emergency Hospital, Romania

Herein we present a very rare case associating a non-functioning pituitary adenoma (PA) with neurofibromatosis type 1 (NF1). Our female patient presented at the age of one bilateral telarche and at the age of five she developped adrenarche, pubarche and menarche. FH and FSH values are those for adults $(2,1$ and $0,1 \mathrm{mIU} / \mathrm{mL}$ respectively), estradiol level was elevated $(18.1 \mathrm{pg} / \mathrm{mL})$ and bone age was slightly increased. Initial imaging investigation showed a normal sella turcica on lateral skull X-ray but CT objectived a milimetric cystic lesion above the pituitary gland, confirmed by MRI.The patient was diagnosed as a real precocious puberty and start suppressive gonadotrophin therapy. Yearly clinical, hormonal and image monitoring showed no progress of the lesion so periodic active surveillance was our preferable attitude. At the age of 12 the appearance of café-au-lait spots which have progressively invaded the trunk, the face and the upper limbs was noted; to these, axillary freckling and pathognomonic presence of Lisch nodules was added. Therefore the diagnosis of our patient was completed with that of neurofibromatosis type 1 . After 16 years the patient continued annually her followup in both pediatric and endocrinology clinics performing periodic clinical, biological and MRI examinations in order to monitor both lesions. Her physical and intellectual development is appropriate, attends a vocational school and have an almost normal social existence. Comorbidities in pediatric patients, wherein frequently at least one component is congenitally, may have an unusual course with higher risks of complications and are less responsive to current therapies. Among these eventualities the association between an endocrine neoplasm as PA and a genetic disorder like NF1 are true pathological exceptions. To our knowledge the present case represent the first appearing in early childhood.

\section{1}

\section{Endocrine \& Diabetes}

Short Stature - Most Common Complication in Children Born Small for Gestational Age (SGA) - Our Experiences

Aleksandra Janchevskal ${ }^{1}$, Zoran Gucev ${ }^{1}$, Liljana Tasevska-Rmush ${ }^{2}$, Olivera Jordanova', Aspazija Sofijanova ${ }^{I}$, Velibor Tasic ${ }^{I}$

${ }^{1}$ Medical Faculty, University Children's Hospital, Macedonia

${ }^{2}$ Laboratory of Molecular medicine, University Children's Hospital, Macedonia

\section{Background}

Children born with low birth parameters or small for gestational age (SGA) might had influence in prevalence and outcome in associated conditions or congenital ano-malies which might lead to chronic diseases in adulthood.

\section{Objective}

Growth failure and short adult height are most common expected complications in children born small for gestation age.

\section{Methods}

Clinical birth and anthropometric data were investigated in 100 SGA born children and compared with children born appropriate for gestational age (AGA). An analyzed data were taken during patient's first visit in the study. Auxology parameters were estimated with precise equipment specialized for pediatric population. We confirmed certain diseases and related conditions by applying an appropriate diagnostic protocols and followed with an adequate treatment.

\section{Results}

A 100 SGA born children, 40 boys and 60 girls, were born with mean BW ( -2.51 SDS ) and BL ( -1.46 SDS ) in $39.25 \mathrm{GW}$. An analyzed data of our SGA patients revealed: mostly of them, $65 / 100$ remained short $(-2.87$ SDS), but $35 / 100$ caught up growth ( 0.20 SDS), in $7 / 100$ children were diagnosed congenital anomalies of kidneys and urinary tract (CAKUT), 4/100 had cystic fibrosis (CF), 4/100 children were obese, but two of them had hyperinsulinemia and insulin resistance, $3 / 100$ girls manifested signs of precocious puberty, and 1 patient developed psychosomatic impairment and one girl had autoimmune thyroid disease.

\section{Conclusions}

Herein we presented several associated conditions in our cohort of 100 SGA born children from Macedonia. Mostly of them, $65 \%$ did not achieved catch up growth after 2nd year and remained short.

Key words: small for gestational age, catch up growth, short stature, associated conditions and congenital anomalies 
119

\section{Endocrine \& Diabetes}

Etiologies of Congenital Hypothyroidism: 15-Year Experience in A Tertiary Care Medical Center in Southern Thailand

Somchit Jaruratanasirikul ${ }^{l}$, Jutarat Piriyapan ${ }^{l}$, Tansit Sangkaew ${ }^{l}$, ${\text { Waricha Janjindamai }{ }^{I}, \text { Hutcha Sriplung }}^{1}$

${ }^{1}$ Pediatrics, Prince of Songkla University, Thailand

\section{Background}

Congenital hypothyroidism $(\mathrm{CH})$ is one of the common causes of mental retardation which can be prevented by early detection and treatment with thyroxine supplementation. In Thailand, neonatal TSH screening was implemented nationwide in 2003.

\section{Objectives}

To determine the etiologies of $\mathrm{CH}$ before and after neonatal TSH screening program implementation.

\section{Method}

The medical records of patients aged $0-15$ years with the diagnosis of congenital hypothyroidism during 1995-2012 were retrospectively reviewed. Results: During the study period, 416 children were diagnosed as $\mathrm{CH}: 76(18.3 \%)$ with transient $\mathrm{CH}, 147$ (35.3\%) with mild $\mathrm{CH}$, and 193 (46.4\%) with severe $\mathrm{CH}$. Thyroid scans performed in 306 patients with permanent $\mathrm{CH}$ revealed eutopic thyroid in 121 cases (39.6\%), ectopic thyroid in 102 cases $(33.3 \%)$, athyreosis in 72 cases $(23.5 \%)$, and hypoplasia of the thyroid in 11 cases (3.6\%) Comparison of etiologies and types of $\mathrm{CH}$ between before TSH screening implementation (1995-2004) and after the TSH screening implementation (20052012) found that transient $\mathrm{CH}$ and mild permanent $\mathrm{CH}$ were significantly more commonly diagnosed after TSH screening implementation and eutopic thyroid or thyroid dyshormonogenesis was a significantly common etiology of $\mathrm{CH}$ diagnosis by thyroid scan (p0.001). Transient $\mathrm{CH}$ was significantly more frequently diagnosed in preterm than term neonates (p0.001) with significantly lower levels of screened neonatal TSH and confirmed TSH than the mild and severe permanent $\mathrm{CH}$ cases (P0.001).

Conclusions

Neonatal TSH screening increase the number of cases of transient $\mathrm{CH}$ and mild form $\mathrm{CH}$ detected resulting in early treatment to prevent permanent brain damage in cases with less severe $\mathrm{CH}$.

\section{0}

\section{Endocrine \& Diabetes}

A Paediatric Safety Checklist Based on the Principles of the WHO Surgical Safety Checklist

Victoria John ${ }^{1}$, Beshlie Richards ${ }^{1}$, Torsten Hildebrandt ${ }^{1}$

${ }^{T}$ Department of Paediatrics, Princess of Wales Hospital, UK

\section{Background}

A 3-year old child presented with apparent respiratory problems. The underlying problem of diabetic ketoacidosis was missed and diagnosis was delayed. This was an isolated incident comparable to a surgical 'Never Event'. We hypothesised that a Paediatric Safety Checklist based on the principles of the WHO Surgical Safety Checklist ${ }^{1}$ can address this. Objective

To determine whether a Paediatric Safety Checklist can be integrated successfully.

Methods

Potential barriers were addressed, including clinical engagement, active and passive resistance from staff and practicalities in relation to the current system. Five PDSA cycles were completed from September 2014 to June 2015, with intermittent interventions. A further PDSA cycle was undertaken in January 2017. A staff questionnaire was performed in September 2014 during initial introduction and repeated in February 2017.

\section{Results}

The first PDSA cycle in 2014 showed $55 \%$ compliance rate. A fall to $17 \%$ was seen on the second data collection. After interventions compliance increased to $67 \%$ and remained at $62 \%$ and $67 \%$ respectively. In January 2017 none of the checklists in a sample of 40 were filled appropriately.

In $201473 \%$ of doctors felt that the Paediatric Safety Checklist improved patient safety, compared with $83 \%$ in 2017. One hundred percent of nurses agreed with this in 2014, compared to $53 \%$ in 2017. In 2017 $52 \%$ of staff lacked awareness of the trigger case and $67 \%$ stated this knowledge increased their opinion of checklist importance.

Discussion

We were able to introduce the Paediatric Safety Checklist in 2014; three years later however the checklist has stopped working. There is a lack of compliance despite previous and current positive feedback. Reasons appear to be the absence of champions and a lack of clarity by staff in regards to what is expected. The questionnaires showed that staff perceptions and attitudes are key to the project's success.

\section{References}

1. Haynes AB et al. A surgical safety checklist to reduce morbidity and mortality in a global population. N Engl J Med 2009; 360:491-499.

\section{7}

\section{Endocrine \& Diabetes}

The Occurrence of Dysembryogenesis Variants in Uzbek Girls with SHereshevsky-Turner Syndrome Nilufar Ibragimova ${ }^{I}$, Rustam Mukhamedov ${ }^{I}$, Malika Mirkhaidarova ${ }^{1}$ ${ }^{T}$ Laboratory of Genetics, Institute of Bioorganic Chemistry of the Academy of Sciences of Republic Uzbekistan, Uzbekistan

\section{Aim}

To determine the rate of occurrence dysembryogenesis variants in Uzbek girls with Shereshevsky-Turner syndrome.

\section{Methods}

We examined 46 Uzbek girls with Shereshevsky-Turner syndrome aged from 8 to 18 (mean age: $14.6 \pm 0.38$ years) with mean height $132.8 \pm 1.21 \mathrm{~cm}$. Clinical-cytogenetic investigations were performed with ultrasound examination of small pelvis organs, heart and kidneys.

\section{Results}

One hundred per cent growth delay was found in all patients $(-3.9 \pm 0.26 \mathrm{SDS})$, being marked in puberty age girls $(-4.3 \pm 0.54 \mathrm{SDS})$. Gonadal dysgenesis was found in $93.7 \%$. Cytogenetic investigation helped diagnose monosomy and mosaicism in $25(54.3 \%)$ and $21(45.7 \%)$ girls. We have managed to reveal the following variants of dysembryogenesis. Congenital hearing loss and otopyosis were found in $40 \%$ of examinees with hearing problems; $2 \%$ of girls having congenital heart disease (aortic stenosis, patent ductus arteriosus). Twenty-seven percent of examinees had kidney pathologies, such as, kidney salt masses, supplemental chord and solitary kidney; in $24 \%$ psychomotor retardation and oligophrenia being registered. Autoimmune thyroiditis was found in $37.4 \%$ of girls with Shereshevsky-Turner syndrome to be the Uzbek population feature. Ultrasound examination demonstrated III degree uterine hypoplasia in $66.3 \%$ with mosaicism; $90 \%$ of patients with monosomy had ametria. Spontaneous puberty was observed in $9.7 \%$ of girls only.

\section{Conclusions}

Irrespective to chromosomal aberrations in Uzbek girls with Shereshevsky-Turner syndrome $100 \%$ growth delay was found, $93.5 \%$ having gonadal dysgenesis. The most pronounced manifestations of phenotypic signs and variants of dysembryogenesis were found in girls with monosomy. Percentage of disturbances in hearing system $(40 \%)$ and kidneys $(27 \%)$ was the highest. The feature of Uzbek population, autoimmune thyroiditis was registered in $37.4 \%$ of girls. 
548

\section{Endocrine \& Diabetes}

Situation of iodine deficiency in the Tomsk region, Russia in 15 years Oxana Oleynik ${ }^{l}$, Julia Samoylova ${ }^{I}$, Elena Yurchenko ${ }^{1}$, Sergey Zinchuk ${ }^{2}$ ${ }^{T}$ Department of endocrinology and diabetology, Siberian State Medical University, Russia

${ }^{2}$ Central research laboratory, Kemerovo State Medical University, Russia

The aim of this study is a comparative analysis of the epidemiological situation of iodine deficiency in the Tomsk region from 1998 to 2014 year. Nine thousand nine hundred one and 15174 school-age children were surveyed in the city of Tomsk. Medical history, anthropometric measurements were analyzed for all students, thyroid ultrasonography was performed with portable scanner "Aloka SSD 500" with linear transducer frequency of 7.5 MGz. The excretion of inorganic iodine in urine was determined at 264 children in 1998 year and 120 children in 2014 year respectively, in a single urine portion using ceriumarsenit method. Analysis of TSH results was done to 10717 newborns in 1998, and to 15091 newborns in 2014 in capillary blood samples taking on 4-5 days after birth. Neonatal TSH were determined by fluorometric immunoassay kits using TSH-Neonatal: Delfia, Finland. According to our survey median urinary iodine of schoolchildren was $72.5 \mathrm{mcg} / \mathrm{l}$ in 1998, and $92 \mathrm{mcg} / \mathrm{l}$ in 2014, which shows both the increase in iodine provision of the population over 15 years on $27 \%$, and keeping the level of urinary iodine, wich corresponds to mild iodine deficiency according to WHO criteria. Increase of level of TSH5MU/1 was registered in $11.7 \%$ of newborns in 1998 and in $7.7 \%$ of newborns in 2014, which shows the mild degree of iodine deficiency. Comparative analysis of the epidemiological situation of iodine deficiency in Tomsk for 1998 and 2014 shows iodine provision growth on $27 \%$ over 15 years, also the decrease in frequency of neonatal hyperthyrotropinemia in 1.5 times, but all criteria stacked indicate mild degree of iodine deficiency in Tomsk region.

662

\section{Endocrine \& Diabetes}

Effects of iodine supplementation on physical and psychomotor development in young children and their neurological status Anna Shlieienkova ${ }^{2}$, Marina Mamenko $^{2}$

${ }^{\top}$ Internal medicine, $\bar{V}$. N. Karazin Kharkiv National University, Ukraine

${ }^{2}$ Paediatrics № 2, Shupyk National Medical Academy of Postgraduate Education, Ukraine

Iodine is an essential element for the synthesis of thyroid hormones. Thyroid function is crucial to the metabolism of almost all tissues and is critical for the development of the central nervous system in the fetus and children.

OBJECTIVE

To evaluate effects of iodine supplementation on physical and psychomotor development in young children and their neurological status.

\section{MATERIALS AND METHODS}

Target group: 118 children 0-3 years old from orphanage. Methods: physical and psychomotor development accession; dietary iodine intake evaluation by urinary iodine concentration (Sandell-Kolthoff reaction); thyroid status by TSH, TT4, TT3 serum-based measuring (IMA). Iodine intake: infant formula (iodine concentration $100 \mu \mathrm{g} / \mathrm{l}$ ) and additional iodine supplementation in 51 children by drugs ( $50 \mu \mathrm{g}$ per day for 6 months).

RESULTS

All children were accepted into an orphanage at the age 2.5-3.5 months. They had burdened prenatal history $(100 \%)$, decreased physical development $(44.9 \%)$, deficiency in psychomotor development $(95.7 \%)$, decreased coefficient of neuropsychological development (82 points) and neurological signs $(94.1 \%)$. The most common was the signs of static, kinetic, mental and prespeech retardation $(49.2 \%, \mathrm{p}<0.001)$. Median urinary iodine excretion was $56.5 \mu \mathrm{g}$ per day. Median TSH level was $3.21 \mathrm{mU} / \mathrm{l}$ [QR: 3.1; 3.8]. Negative linear correlation by PPMCC between TSH levels and coefficients of neuropsychological development was detected $(\rho=-0.436$, p 0.001$)$. Iodine supplementation per 6 months reduced median TSH level up to $1.96 \mathrm{mU} / 1$ [QR:
$1.77 ; 2.01](\mathrm{p}<0.001)$ vs median TSH level in control group $2.99 \mathrm{mU} / 1$ [QR: $2.43 ; 3.98], \mathrm{p}<0.001$. That resulted in improvement of anthropometry indicators $(92.3 \%, \mathrm{p}<0.001)$ and psychomotor development $(50 \%, \mathrm{p}<0.001)$. The coefficient of neuropsychological development increased (up to 92 points). The frequency of neurological signs reduced $(28.9 \%$, p $<0.001)$.

\section{CONCLUSIONS}

Iodine supplementation in young children with developmental retardation improves their growth, cognitive, emotional, motor, and social capacities, reduces neurological dysfunction rate.

\section{3}

\section{Endocrine \& Diabetes}

Quality of Life in Women With Turner Syndrome After Pediatric Growth Hormone Therapy

Michael Yafi ${ }^{l}$, Marianna Raia ${ }^{2}$, Jacqueline Hecht ${ }^{l}$, Hope Northrup ${ }^{1}$, Michael Gambello ${ }^{3}$, Patrick Brosnan ${ }^{1}$

${ }^{1}$ Pediatrics, The University of Texas Health Science Center at Houston, USA ${ }^{2}$ Genetics, Cousyl, USA

${ }^{3}$ Medical Genetics, Emory University, USA

\section{Background}

Short stature is the most common feature of Turner Syndrome (TS). Growth hormone $(\mathrm{GH})$ therapy in TS has shown to improve final height. The effect of improving the quality of life (QOL) by improving the final height on short children in general and girls with TS in particular has been controversial. Women with TS may have other TS related comorbidities that may affect their QOL.

Objectives

- To determine whether use of GH therapy in TS women affected the QOL

- To determine if improvement in QOL scores (post treatment) are due to increased height or change in social behavior, increased education or other factors.

Method

Four hundred surveys were mailed out to members of TS Society in the USA. The survey included questions about history of GH therapy, height, income, education, marital status and quality of life index developed by Ferrans and Powers to evaluate:

1-Health and functioning

2-Social and economics

3-Psychological and spiritual life

4-Family status

Results

- $174(43.5 \%)$ surveys were returned.

- $48(27.6 \%)$ were GH treated while $126(72.4 \%)$ were untreated.

- No difference in QOL between the treated versus non-treated groups.

-Overall satisfaction was not significantly different before and after GH treatment.

-Some observational outcomes of well-being were related to physical strength, appearance, ability to make friends and obtaining a higher income. Conclusion

Several studies about children treated with GH have shown no significant improvement in QOL. This study involving 48 TS women has led to the same conclusion. Improving final height in TS girls allows them to attain better functioning in daily activities. The $\mathrm{GH}$ treated group was younger than the non-treated group reflecting the natural history of GH use in TS. Future studies are warranted to help understand the effect of GH- related height gain on QOL by reevaluating different generations of TS women.

\section{1}

\section{Endocrine \& Diabetes}

Cytochrome P450 Oxidoreductase Deficiency PORD: A case report Hector Lantigua ${ }^{l}$, Nunilo Rubio ${ }^{l}$, David Rodriguez-Buritica ${ }^{l}$, Amir Khan ${ }^{1}$, Michael Yafi ${ }^{1}$

${ }^{I}$ Pediatrics, The University of Texas Health Science Center at Houston, USA 


\section{Background}

A rare and the newest form of congenital adrenal hyperplasia is Cytochrome P450 oxidoreductase deficiency (PORD). There has been close to 100 cases reported.

\section{Objective}

We report a case of a newborn with several characteristic features of POR deficiency.

\section{Case description}

Patient is a full term new born product of first pregnancy of an 18 year old mother. Pregnancy uncomplicated, normal estradiol on second trimester screening, mother report increased acne. Delivered via C-section due to failure to progress of labor, APGAR 2, 5, 5 at 1, 5 and $10 \mathrm{~min}$, respectively. Small for age, weigh $2.726 \mathrm{Kg}$ (99th percentile) anterior fontanelle, down slanting palpebral fissures, proptosis, Long palpebral fissures, up turned nose, Flat nasal bridge, retronagtia, large tongue. Microphallus $1.5 \mathrm{~cm}$ (5th percentile) with hypospadias with urethral opening located in the glans, testicles descended. Elbows in fixed position. Second toe this longer than 1st. Increased separation between 1st and 2nd toe, 3rd, 4th, and 5th short. Unremarkable family history with no consanguinity. X-ray demonstrated bilateral Radio-humeral synostosis. Intravenous $250 \mathrm{mg}$ Cosyntropin stimulation test with Cortisol $6.7 \mathrm{ug} / \mathrm{dL}$ and $15.1 \mathrm{ug} / \mathrm{dL}$ at $0 \mathrm{~min}$ and $60 \mathrm{~min}$. No cardiovascular, electrolytes or glucose abnormalities. Sanger sequencing of the $P O R$ gene demonstrated two pathogenic variants c.859GC(p.Ala287Pro) and c.651_653del (p.Glu217del.)

\section{Conclusion}

We demonstrated several features, as well two pathogenic variants in compound heterozygous state of P450 Oxidoreductase (POR) gene in an individual with blunt cortisol production that might require steroid supplementation during stress, as was advised in this case.

622

\section{Endocrine \& Diabetes}

A case of pheochromocytoma

Daniela Ovadia-Perla ${ }^{1}$, Michael Yafi ${ }^{1}$

${ }^{T}$ Pediatrics, The University pf Texas Health Scinece Center at Houston, USA

\section{Background}

Pheochromocytoma is a rare neuroendocrine tumor that arises from the chromaffin cells, originates in the adrenal gland, particularly in the medulla.

Pheochromocytomas can produce large quantities of catecholamines (such as epinephrine and norepinephrine) causing severe hypertension.

The case

A 6-year-old male presented with a 2 months history of mood swings associated with severe headaches, facial flushing, sweating, increased water intake and urination. CT scan of abdomen and pelvis without contrast revealed a $2.5 \times 2.8 \times 3.1 \mathrm{~cm}$ heterogeneous right adrenal mass. Patient was admitted to the pediatric intensive care unit due to severe hypertension and was started on Nicardipine drip and phenoxibenzamine.

Initial studies revealed a 24-hour urine Vanillylmandelic acid of $21.6 \mathrm{mg} /$ day (Ref. Range 0.0-7.5), 24-hour urine Normetanephrine was $7059 \mathrm{ug} /$ 24 hrs (Ref. Range 13-252), urine Normetanephrine was 5679 pg/ml (Ref. range 10-25) and urine Metanephrine was $57 \mathrm{pg} / \mathrm{ml}$ (Ref. range 2-15). Subsequent imaging studies revealed an MRI of the abdomen that showed heterogeneously enhancing $3.2 \mathrm{~cm}$ right suprarenal mass and no liver lesion is identified however MIBG Scintigraphy showed tracer uptake in the right upper quadrant corresponding with adrenal mass seen by CT, compatible with pheochromocytoma, additionally a smaller focus of mild uptake within hepatic segment. Chromosomal microarray was sent to rule out an underlying genetic disease.

The patient underwent surgery to remove the tumor, pathology confirmed pheochromocytoma.

Patient remained stable after surgery he has been off antihypertensive medication.

\section{Discussion}

Pheochromocytoma are exceedingly rare in children. Phechromocytoma has been associated several genetic syndromes such as in multiple endocrine neoplasia, Type I1 (MEN-11), Neurofibromatosis (Von Recklinghausen's disease), Von Hippel Lindau syndrome and cerebellar hemangioblastomas therefore, genetic work up is essential.

Most of these tumors do not spread and are cured with surgery, however in rare cases it can cause metastasis. Recurrence is unusual but patients need to be monitored lifelong.

\section{8}

\section{Endocrine \& Diabetes}

Post-operative use of human chorionic gonadotrophin (u-hCG) in patients treated for intrabdominal undescended testes

Nicola Zampieri ${ }^{l}$, Marta Peretti ${ }^{l}$, Federica Bianchi ${ }^{1}$, Simone Patanè ${ }^{l}$, Virginia Murri ${ }^{2}$, Francesco Camoglio ${ }^{1}$

${ }^{I}$ Pediatric Surgical Unit, Azienda Ospedaliera Universitaria Integrata, University of Verona, Italy

${ }^{2}$ Department of Pediatrics, Azienda Ospedaliera Universitaria Integrata, Italy

\section{INTRODUCTION}

Testicular volume and function improvement of intrabdominal testes after orchiopexy is still under discussion. The aim of this study is to report our experience with post-operative use of human chorionic gonadotrophin to achieve higher testicular volume and function, respect to untreated patients $/ \mathrm{h} 4$ METHODS

A prospective study was done using subjects who underwent orchidopexy between Sptember 2010 and September 2016 for unilateral intrabdominal undescended testes. All patients were treated by the same surgeon with laparoscopic one-stage Fowler-Stephens technique. After surgery ( 2 weeks) those patient parents who accepted to use hormonal therapy, had to follow a 6 weeks sheme. Patients received subcutaneous 500UI (Gonasi-HP) weekly. A follow-up was performed at the end of therapy and 6 months later.Testicular volume was measured at each visit by ultrasound and compared with the untreated one.

\section{RESULTS}

Fortyfive patients were enrolled and treated with a mean age of $18.0 \pm 9.7$ months. Thirty-two patients received post-oprative hormonal therapy. There were no cases of adverse effects nor droupout. All patients completed follow-up. There were no cases of testicular atrophy in both groups. At 6 months among treated patients $26(81 \%)$ subjects achieved normal testicular size while the other had still smaller volume. Among untreated patients, 6 (46\%) subject achieved normal testicular size. (p0.05)

\section{CONCLUSIONS}

Despite the role of hormonal therapy is still under discussion, especially for post-operative treatment, our results suggest that it is safe and useful to improve testicular volume; treated testes have also a good stifness respect to untreated testes $/ \mathrm{h} 4$

\section{6}

\section{Gastroenterology \& Nutrition}

Assessment of Helicobacter Pylori Colonization in Children Tonsillar Tissues

Mandana Rafeey ${ }^{1}$, Yalda Jabbari Moghaddam ${ }^{2}$

${ }^{T}$ pediatric gastroenterology, Liver and Gastrointestinal Research Center, Tabriz University (Medical Sciences), Iran, Iran

${ }^{2}$ Otorhinolaryngology, Liver and Gastrointestinal Research Center, Tabriz University (Medical Sciences), Iran

\section{Objective}

The objective was to survey the results of RUT (rapid urease test) in children tonsillar tissues. 


\section{Methods}

In a prospective clinical study 285 children (4-14 years) tonsillar tissue tested with RUT (rapid urease test) and histopathologic biopsy and simultaneously serum IgG Helicobacter pylori level was measured for all patients.

\section{Results}

One hundred and thirteen patients $(39.6 \%)$ were positive to $\mathrm{H}$. pylori in histopathologic examination. Forty patients (14\%) had positive RUT and 15 patients had positive serum IgG anti-H. pylori level. In 40 patients the results in both histopathology and RUT were positive $(P=0.000)$ although in 172 patients the results in both histopatologhic and RUT were negative $(P=0.000)$.

\section{Conclusions}

This study showed that H. pylori was present in tonsillar tissue and RUT is not sensitive enough for diagnosis of H. pylori in tonsillar tissue. Indicating that $\mathrm{H}$. pylori has a possible role in reservoir of $\mathrm{H}$. pylori in children.

\section{7}

\section{Gastroenterology \& Nutrition}

Plasma Fatty Acids and Their Binding Proteins in Preterm Infants Barbara Koscielniak ${ }^{1}$, Nina Mol ${ }^{2}$, Przemko Kwinta ${ }^{2}$, Przemyslaw Tomasik ${ }^{l}$ ${ }^{T}$ Department of Clinical Biochemistry, University Children's Hospital, Jagiellonian University Medical Collegue, Poland

${ }^{2}$ Department of Pediatrics, University Children's Hospital, Jagiellonian University Medical Collegue, Poland

\section{Background}

Fatty acids and their binding proteins are involved in intracellular signaling inflammatory responses, hemostasis and central nervous system development. Objective

Postnatal deficits of fatty acids in preterm infants are known, but no one determined the levels of fatty acids binding proteins in preterm babies in relation to used diet during first month of life.

Materials

Forty-two premature infants $\leq 34$ weeks hospitalized in the Children's University Hospital in Krakow between March 2014 and September 2016 were enrolled in the study. The children were divided into three subgroups: nursed during staying in the department (55\%), feeding with the breast milk only at the beginning (14\%) and children feeding from the beginning with modified milk (31\%). The control group consisted of 10 healthy, term newborns. Blood samples were drawn at the first day of hospitalization and one month later. Free fatty acids (FFA) concentrations were measured using Roche kit, fatty binding protein 1 (FABP1) and fatty acid binding protein 4) were determined using ELISA kits.

\section{Results}

We have found higher FFA concentration in preterm infants than in term infants (median $1.16 \mathrm{mmol} / \mathrm{l}$ vs $1.01 \mathrm{mmol} / \mathrm{l} ; p=0.012$ ) in the prenatal period, where as in the second month of life there were no statistical differences in FFA concentrations in preterm infants and in term infants. Also type of feeding no influences FFA concentration in preterm neonates. We noted diminution in FABP4 serum concentration in the premature infants feeding with breast milk after one month of hospitalization $(p=0.017)$. Additionally we observed that premature nursed infants had lower serum FABP4 concentration in the second month of life than in the perinatal period $(p=0.022)$. Conclusions

Prematurity is connected with disturbances in FABP4 plasma levels where FABP1 concentrations remain similar to those observed in term infants.

163

\section{Gastroenterology \& Nutrition}

Pediatric Oral Biopsies in Thailand: A 15-year Retrospective Review from a Medical Teaching Hospital

Mana Taweevisit ${ }^{1}$, Paul Thorner ${ }^{1,2}$

${ }^{T}$ Pathology, Faculty of Medicine, Chulalongkorn University, Thailand

${ }^{2}$ Pathology and Laboratory Medicine. Hospital for Sick Children and

University of Toronto, Canada

\section{Background}

Reports on the spectrum of oral pathology in children are almost totally based on biopsies submitted to dental facilities. Specimens submitted to pathology laboratories in medical institutions could show a different spectrum of disease, including entities under-represented in dental studies.

\section{Objective}

To determine the prevalence of oral lesions in children sent to pathology service in the medical teaching hospital.

\section{Methods}

Oral biopsy records from pediatric patients (16 years old) were retrieved from the Pathology archives in Chulalongkorn University Hospital over a period of 15 years (2000-2014). Age, gender, location and pathological diagnoses were collected. The age range was divided into 6 years, 6-12 years and 12 years. Lesions were categorized as inflammatory/reactive, tumor/tumor-like and cystic lesions.

\section{Results}

Of a total of 3,887 cases, 230 biopsies ( $6 \%$ ) were found. Most lesions were in the inflammatory/reactive group $(n=128 ; 56 \%)$, followed by tumor/tumorlike $(n=95 ; 41 \%)$ and cystic lesions $(n=7 ; 3 \%)$. Most cases occurred in the 12 year age group. Mucocele was the most common lesion $(n=87 ; 38 \%)$, followed by hemangioma $(n=19 ; 8.3 \%)$ and nevus $(n=13 ; 5.7 \%)$. Lip was the most common location. The predominance of mucocele is similar to reports from various countries, indicating prevalence is not affected by geographic differences. The proportion of malignant tumours $(4.8 \%)$ was higher compared to most studies (1-2\%). In contrast, odontogenic cysts and odontogenic tumours were rare $(2.8 \%$ and $1 \%$, respectively), compared to published studies (7-35\% and 2-21\%, respectively).

\section{Conclusion}

This study from a medical institution shows a somewhat different spectrum of pediatric oral biopsies compared to dental institutions, which could reflect institutional staffing, practice patterns and the type of facility in which specific lesions are managed.

\section{1}

\section{Gastroenterology \& Nutrition}

\section{In Adequate Vitamin D levels In Children Of Northern Indian} Province Of Punjab

Deepak bhat ${ }^{l}$, Satinder Kaur ${ }^{2}$, Gurdeep Singh Dhooria ${ }^{l}$

${ }^{T}$ Pediatrics, Dayanand Medical College and Hospital, India

${ }^{2}$ Biochemistry, Dayanand Medical College and Hospital, India

\section{Background}

Hypovitaminosis D is common in children in developing countries. Though majority of population in India lives in areas receiving ample sunlight throughout the year,vitamin D deficiency is very common in all the age groups across the country.

\section{Objective}

This study was undertaken to know the incidence of vitamin D deficiency in Punjabi children.

\section{Methods}

This was a retrospective study in which case records of all the children whose 25-(OH)D levels were send from pediatric OPD department of Dayanand medical college and hospital between Jan 2011 and Jan 2015.All the patients were divided into four age groups.Group 1:0 1 years, Group II:1-6 years, Group III:7-12 years and Group IV:12 years. $25(\mathrm{OH}) \mathrm{D}$ levels were categorized as deficient if $20 \mathrm{ng} / \mathrm{ml}$, insufficient if between $20-30 \mathrm{ng} / \mathrm{ml}$, sufficient if between 30 $60 \mathrm{ng} / \mathrm{ml}$ and toxic if $70 \mathrm{ng} / \mathrm{ml}$. Values less than $4 \mathrm{ng} / \mathrm{ml}$ were categorized as severe deficiency.

\section{Results}

More of male children underwent vitamin D level checkup as compared to female.Number of patients has kept on increasing in each age group.In younger age group more of male children reported for investigation whereas in adolescents more of female children got investigated.No. Of children with severely deficient vitamin D levels has also shown a 
decreasing trend over these four years.Cildren with high levels of Vitamin $\mathrm{D}(70 \mathrm{ng} / \mathrm{ml})$ has also increased over the years.Vitamin D deficiency has shown a decreasing trend over these years.

\section{Conclusions}

The world is currently facing an unrecognised and untreated pandemic of vitamin $\mathrm{D}$ deficiency.Sensitising pediatricians to recognise and treat this pandemic would have great impact on child health in the $21 \mathrm{st}$ century.Widespread subclinical and pre rachitic vitamin $\mathrm{D}$ deficiency in children should be diagnosed by serum $25(\mathrm{OH}) \mathrm{D}$ levels and these levels should be maintained above $20 \mathrm{ng} / \mathrm{dl}$ to obtain optimal health benefits.

196

\section{Gastroenterology \& Nutrition}

Identifying Nutritional Risk in Hospitalized Children: Using Anthropometry, Comparing with Laboratory and Nutritional Screening Tools

Guniz Yasoz ${ }^{1}$, Deniz, Ertem ${ }^{1}$

${ }^{T}$ Pediatric Gastroenterology, Marmara University, Turkey

\section{Introduction}

Malnutrition in children is one of the most serious health problems in the world. Especially, hospitalized children are under the risk of malnutrition. It is recommended that all children admitted to hospital should be assessed by anthropometry and determined their nutritional status. We aimed to identify the nutritional risk in hospitalized children by using anthropometric parameters, comparing with laboratory and STRONGkids and PYMS.

Methods

Five hundred three patients, aged 1 month-18 years, were enrolled. Anthropometric measurements were taken and converted to z-scores. STRONGkids and PYMS tools were completed for all patients. Age, race, department for admission, disease, underlying disease, laboratory values were taken. When discharged, weight measurement was repeated. Results

$225(\% 44.7)$ were female, 278 (\%55.3) were male. Age distribution was $101(\% 20.1)$ for $0-2$ years, $102(\% 20.3)$ for $2-5$ years, $138(\% 27.4)$ for 5 10 years and $162(\% 32.2)$ for $10-18$ years. The average length of hospital stay was 9.1 days. Acute malnutrition using W/H z-score was \%14.8, W/A z-score was \%10.6, BMI/A z-score was \%15.5. Chronic malnutrition was \%9.4. When compared antropometric parameters in admission and discharge, there was no statistically significant difference. Patients with chronic malnutrition have lower serum albumin and hemoglobin levels. In general, STRONGkids tool was successful in identifying patients with $z$-score -2 , but patients with $z$-score +2 , but failed in patients with $z$-score $\geq-3$ and -2 , in fact the tool overestimated this group.

Conclusion

Hospital stay does not affect the nutritional status adversely. Actual nutritional status isn't a predictor for hospital acquired malnutrition. Children with underlying disease are more prone to developing malnutrition, attention should be paid to these children. Laboratory tests aren't good predictors. In this study, we confirmed STRONGkids underestimate high nutritional risk patients. Instead, PYMS tool was successful in identifying high risk patients. In screening patients with high nutritional risk, we suggest using PYMS tool with anthropometric measurements.

\section{4}

\section{Gastroenterology \& Nutrition}

Effect of Pre/Probiotic Mixture in Children with Nonalcoholic Fatty Liver Disease: A Randomized Controlled Trial

Voranush Chongsrisawat ${ }^{1}$, Hansamon Poparn ${ }^{1}$, Susheera Chatrproedprai ${ }^{I}$, Kanokwarn Sornsiri ${ }^{2}$, Sombat Treeprasertsuk ${ }^{2}$

${ }^{1}$ Department of Pediatrics, Faculty of Medicine, Chulalongkorn University, Thailand

${ }^{2}$ Department of Medicine, Faculty of Medicine, Chulalongkorn University, Thailand

\section{Background}

The prevalence of pediatric nonalcoholic fatty liver disease (NAFLD) has been increasing worldwide in parallel with the obesity epidemic. Dysbiosis of gut microbiota is implicated in the pathogenesis of NAFLD. Objective

This study aimed to investigate the effect of pre/probiotic mixture treatment in children with NAFLD.

\section{Methods}

This was a randomized, double-blind, placebo-controlled trial conducted between February and October 2016. NAFLD was defined by controlled attenuation parameter (CAP) value of greater than $250 \mathrm{~dB} / \mathrm{m}$ without other causes of fatty liver. Participants received either a mixture of chicory inulin, Lactobacillus acidophilus, and Bifidobacterium lactis or placebo for 16 weeks. Body mass index (BMI), serum alanine transaminase (ALT), and liver stiffness measurement (LSM) with simultaneous controlled attenuation parameter (CAP) determination using the FibroScan ${ }^{\circledR}$ were assessed at baseline and after the completion of the intervention. BMI Z-score adjusted for age and sex was determined according to the 2007 WHO child growth references.

\section{Results}

There were 18 (78\% male) and 19 children (58\% male) in intervention and placebo groups. The number of children aged $\leq 9$ and 9 years were not significantly different between the two groups. Baseline mean (SD) of BMI Z-score, serum ALT, CAP, and LSM of enrolled participants were 2.9 (0.7), 51.8 (41.6) U/L, 312.8 (38.3) dB/m, and 6.1 (3.5) kPa. After the completion of the intervention, BMI Z-score significantly decreased in both groups. The reduction of CAP value was significant only in the placebo group $(P=0.04)$. The median between-group difference in change from baseline (interquartile range) for BMI Z-score, serum ALT, CAP, and LSM values were $-0.0(-0.1,-0.1),-1.7(-19.4,16.1) \mathrm{U} / \mathrm{L}$, $5.74(-22.8,34.3) \mathrm{dB} / \mathrm{m}$, and $0.2(-0.5,0.9) \mathrm{kPa}$, which were not statistically significant.

\section{Conclusion}

The present study is unable to demonstrate the beneficial effect of this particular pre/probiotic mixture on BMI and hepatic derangement in children with NAFLD.

\section{3}

\section{Gastroenterology \& Nutrition}

A Retrospective Review of Vitamin D Requests in General Paediatric Practice

Robert Kirwan ${ }^{1}$, Donncha Sheehan ${ }^{1}$, Uzair Khan ${ }^{1}$, Ann-Marie Murphy ${ }^{1}$ ${ }^{T}$ Paediatrics, University Hospital Limerick, Ireland

\section{Background}

Vitamin D testing in Paediatric Medicine is carried out mainly in the context of a child at risk of deficiency. Deficiency is diagnosed by directly measuring serum levels of 25-hydroxyvitamin D. Serum levels $50 \mathrm{nmol} /$ $\mathrm{L}$ are considered normal. Deficiency is considered mild between $25 \mathrm{nmol} /$ $\mathrm{L}-50 \mathrm{nmol} / \mathrm{L}$, moderate between $12.5 \mathrm{nmol} / \mathrm{L}-50 \mathrm{nmol} / \mathrm{L}$ and severe at $12.5 \mathrm{nmol} / \mathrm{L}$. The ranges for defining deficiency were changed between 2011 and 2012 where normal levels were previously between $75 \mathrm{nmol} / \mathrm{L}$ $-200 \mathrm{nmol} / \mathrm{L}$.

\section{Objective}

To review vitamin D testing in an Irish General Paediatric Practice.

\section{Methods}

A history of all vitamin D tests requested by consultant paediatricians in a large general paediatric practice, servicing a catchment area with a population of 100,000 children over the fiveyear time-period from $01 / 07 / 2011$ to $01 / 07 / 2017$ was collected. Patient charts were reviewed and the indications for testing were identified. Results from testing were also collected. Data was analysed using IBM SPSS Statistics package version 23 for Macintosh. 


\section{Results}

A total of 361 tests were requested over the five-year study period. Most of the samples analysed were from female patients. The main indications for testing were: established vitamin D deficiency, presenting with bony pain, neurological issues and failure to thrive. The mean age of the sample was 6 years of age. The majority of cohort had vitamin D levels within the normal range.

Conclusion

The reference range currently in use for vitamin D testing is that from 2012. Using current ranges, few children were deemed deficient. Comparably, three times more were deficient when previous ranges were used. Most patients were not deficient on testing. Forty-six percent of testing was carried out on a patient with previously established deficiency.

\section{9}

\section{Gastroenterology \& Nutrition}

Nuclear tomographic imaging and nutritional rehabilitation for cerebral functions follow up in kwashiorkor

Enas Raafat AbdelHamid

${ }^{T}$ Child Health Department, National Research center, Egypt

\section{Background and aims}

Kwashiorkor represents a group of related disorders known as proteinenergy malnutrition (PEM), it is associated with cerebral changes which consequently would give rise to intellectual and developmental delay. The present study seeks to define and document cerebral changes and abnormalities in a sample of infants with kwashiorkor using brain imaging.

Methods

Prospective cohort study included 20 infants had admitted to the children hospital Ain Shams University, Cairo, Egypt for treatment of Kwashiorkor aged 4-18 months compared to 5 normal weight matched age infants as control. Clinical assessment, laboratory investigation, brain computed tomography and $99 \mathrm{~m}$-HMPAO brain SPECT was done for all infants twice; first on admission and repeated after 10-12 weeks of nutritional rehabilitation.

\section{Results}

Most infants with moderate disease and all infants with severe disease showed significant cerebral changes and hypoperfusion in certain areas of the brain, which significantly resolved after nutritional rehabilitation in all infants except one with sever disease. A positive correlation was found between severity of the disease and extent of cerebral hypoperfusion. Conclusions

Kwashiorkor is associated with cerebral atrophy and impaired dynamic functional activity of the brain that may be detrimental to intellectual development; brain SPECT is more accurate in assessing deleterious effect of PEM on growing brain.

\section{2}

\section{Gastroenterology \& Nutrition}

Computed Tomography of the Abdomen in Children Presenting to the Emergency Department with Abdominal Pain: Indications and Impact on Management

Sarah Salam Rizkl, Samer El Hayek ${ }^{l}$, Rima Hanna Wakim ${ }^{l}$, Nadine Yazbeck ${ }^{l}$ ${ }^{1}$ Department of Pediatrics and Adolescent Medicine, American University of Beirut Medical Center, Lebanon

\section{Background}

Abdominal pain represents a common complaint of children presenting to the emergency department (ED). The underlying etiology ranges from benign disorders to conditions that require life-saving surgeries. Providers are faced with significant challenges in the evaluation of these patients. Since clinical assessment alone is not always sufficient to reach a correct diagnosis; the use of imaging has become an essential tool in evaluating children presenting with abdominal pain. In particular, computed tomography (CT) emerged as one of the most commonly used imaging for this purpose.

\section{Objective}

The objective of this study was to detect the incidence of use of CT of the abdomen and its impact on children presenting to the ED of the American University of Beirut Medical Center (AUBMC) with abdominal pain.

\section{Methods}

This was a retrospective chart review of children between 3 and 15 years of age presenting with abdominal pain to the ED department at AUBMC from January 1, 2014 to December 31, 2014. Patients' demographics, pain severity, duration, associated symptoms, CT abdomen results and management were collected.

\section{Results}

A total of 464 pediatric ED visits to AUBMC were made with the chief complaint of abdominal pain (253 males and 211 females). Up to $10 \%$ of these patients $(N=46)$ underwent an abdominal CT upon their presentation. These patients had mainly severe right lower quadrant pain commonly associated with nausea and vomiting. Appendicitis was the most common pathologic finding seen on imaging $(30.4 \%, N=14)$ followed by mesenteric adenitis $(21.7 \%, N=10)$.

\section{Conclusion}

The most common etiology of abdominal pain in children that require imaging with abdominal CT is appendicitis. Our data demonstrate that more than half of children who underwent $\mathrm{CT}$ did not have any modification in management; this would lead us to call for a thoughtful approach taking into consideration radiation toxicity and cost.

\section{0}

\section{Gastroenterology \& Nutrition}

Inflammatory Response in Chronic Pancreatitis Patients with Exocrine Insufficiency and Ways Correction Horlenko Olesya ${ }^{1}$, Moscal Oksana ${ }^{2}$, Arhiy Emiliia ${ }^{2}$, Prylypko Lyubomyra $^{2}$, Derbak Mariya ${ }^{2}$, Halay Bogdan ${ }^{2}$

${ }^{I}$ Pediatrics with Infectious Diseases, Uzhhorod National University, Medical faculty, Ukraine

${ }^{2}$ Propedeutic Internal Diseases, Uzhhorod National University, Medical faculty, Ukraine

BACKGROUND CHRONIC PANCREATITIS IS A PROGRESSIVE DISEASE WHICH CHARACTERIZED BY ONGOING INFLAMMATION OF THE PANCREAS and linked to the EXOCRINE INSUFFICIENCY interdependence. SYMPTOMS AND COMPLICATIONS VARY FROM PERSON TO PERSON, SO TREATMENTS ARE TAILORED TO EACH PERSON'S NEEDS. MATERIAL AND METHODS. SEVERITY OF INFLAMMATORY AND INFLAMMATORY AND DESTRUCTIVE CHANGES IN THE PANCREAS IN THE PATIENTS WE HAVE STUDIED ACCORDING TO OUR DATE (GENERAL CLINICAL PARAMETERS AND LABORATORY EXAMINATIONS). PATIENTS WITH CYSTIC FIBROSIS WERE EXCLUDED WE MEASURED THE LEVELS OF PRO- AND ANTI-INFLAMMATORY INTERLEUKINS BEFORE AND AT THE END OF INPATIENT TREATMENT. WE HAVE SELECTED 87 PATIENTS WITH CHRONIC PANCREATITIS PAIN AND WITH EXOCRINE INSUFFICIENCY WHO WERE TREATED IN THE GASTROENTEROLOGY DEPARTMENT OF TRANSCARPATHIAN REGION HOSPITAL IN THE 2013-2015 YY. TO ACHIEVE THIS GOAL RESULTS THE LEVELS OF PRO- AND ANTI-INFLAMMATORY INTERLEUKINS BEFORE TREATMENT WERE SIGNIFICANTLY HIGHER THAN NORMAL. THERE ARETNF, IL-6, IL-4 AND IL-10. BUT AFTER TREATMENT PROINFLAMMATORY INTERLEUKINS WERE DECREASED MORE SIGNIFICANTLY THAN INFLAMMATORY. THUS, TNF AND IL-6 LEVELS HAD TENDENCY TO REFERENCE VALUE. LEVEL OF ANTI-INFLAMMATORY IL-4 WAS DECREASED TO REFERENCE VALUE TOO. IL- 10 IN STUDY PATIENTS WAS MODERATELY AND SIGNIFICANTLY INCREASED BUT WAS DECREASED SIGNIFICANTLY AT THE END OF THE 3RD WEEK OF 
TREATMENT. THUS, THE TREATMENT WAS EFFECTIVE NOT ONLY ACCORDING TO TRADITIONAL SUBJECTIVE AND OBJECTIVE EXAMINATION DATA AND WERE CONFIRMED BY CHANGES IN THE LEVELS OF SUCH SENSITIVE INDICATORS AS PRO- AND ANTI-INFLAMMATORY INTERLEUKINS WITH POSITIVE DIRECTION AFTER TREATMENT. CONCLUSION OPTIMIZED TREATMENT SCHEME USING LEDS TO THE NORMALIZATION AND SIGNIFICANT PATHOLOGICAL CHANGE REDUCTION OF OF THE PRO- AND ANTI-INFLAMMATORY INTERLEUKINS LEVELS AND AS RESULT - DECREASE OF THE SEVERITY OF INFLAMMATORY CHANGES IN THE PANCREAS.

\section{1}

\section{Gastroenterology \& Nutrition}

Efficacy of prevention and correction of vitamin D deficiency using therapeutic doses of cholecalciferol in children under three years of age Leonid Klimov $^{I}$, Irina Zakharova ${ }^{2}$, Victoria Kuryaninova ${ }^{1}$, Svetlana Dolbnya $^{l}$, Anna Kasyanova ${ }^{1}$, Anastasia Yagupova ${ }^{I}$, Ekaterina Koroleva ${ }^{2}$ ${ }^{1}$ Pediatrics, Stavropol State Medical University, Russia

${ }^{2}$ Pediatrics, Russian Medical Academy of Postgraduate Study, Russia

\section{Methods}

Ninety-three children aged from 1 month to 3 years living in Stavropol $\left(45^{\circ}\right.$ North). 42 (45.2\%) children were under $12 \mathrm{~m}, 25$ (26.9\%) - from 12 to $24 \mathrm{~m}$, and $26(27.9 \%)$ - from 24 to $36 \mathrm{~m}$. Following determination of the baseline serum concentration of $25(\mathrm{OH}) \mathrm{D}$, the study participants were prescribed ChoC for 30 days: 3,000 IU/day for subjects having $10-19 \mathrm{ng} / \mathrm{mL}$ serum $25(\mathrm{OH}) \mathrm{D} ; 2,000 \mathrm{IU} /$ day and $1,000 \mathrm{IU} / \mathrm{mL}$ for those having $20-29 \mathrm{ng} /$ $\mathrm{mL}$ and $30 \mathrm{ng} / \mathrm{mL}$ serum concentrations of $25(\mathrm{OH}) \mathrm{D}$. Control laboratory tests were performed after a 30-day treatment course with vitamin D (VD). Results

Median baseline level (Me [25Q-75Q]) of calcidiol in the group examined was 25.4 [20.0-31.2] ng/mL. After 1 month use of ChoC according to the suggested dosage regimen, serum $25(\mathrm{OH}) \mathrm{D}$ demonstrated two-fold increase up to 49.1 [40.5-64.5] ng/mL compared to baseline (p0.001). The calcidiol level in subjects receiving VD at the dose of 1,000 IU/day increased from 39.5 [33.4-48.5] to 45.5 [39.6-57.9] ng/mL (p0.05); from 24.9 [22.6-26.5] to 49.2 [41.0-66.6] ng/mL (p0.001) and from 14.1 [12.0$16.1]$ to 53.1 [42.7-68.1] ng/mL (p0.001) in subjects receiving 2,000 IU/ day and $3,000 \mathrm{IU} /$ day doses. The levels of $25(\mathrm{OH}) \mathrm{D}$ within the range of $20-30 \mathrm{ng} / \mathrm{mL}$ were registered in $40(43.0 \%)$ and $8(8.6 \%)$ subjects (p0.001); and the levels within the range of $30-100 \mathrm{ng} / \mathrm{mL}$ were observed in $30(32.3 \%)$ and $80(80.6 \%)$ children, respectively (p0.001). Calcidiol concentration above $100 \mathrm{ng} / \mathrm{mL}$ was revealed in $4(4.3 \%)$ children. The correlation between the daily dose of ChoC and the increment of 25(OH)D as a result of one-month treatment course comprised $r=0.57$ (p0.001). Conclusion

The highest increments of calcidiol were observed when the ChoC doses of 2,000-3,000 IU/day were used

\section{3}

\section{Gastroenterology \& Nutrition}

Treatment for gastroesophageal reflux in children: which technique? Nicola Zampieri ${ }^{1}$, Marta Peretti ${ }^{l}$, Federica Bianchi ${ }^{I}$, Simone Patanè ${ }^{I}$, Virginia Murri ${ }^{2}$, Francesco Camoglio ${ }^{I}$

${ }^{1}$ Pediatric Surgical Unit, Azienda Ospedaliera Universitaria Intergata, Italy ${ }^{2}$ Department of Pediatrics, Azienda Ospedaliera Universitaria Integrata, Italy

Gastroesophageal reflux (GER) is a common problem in infants and children. Pathologic gastroesophageal reflux disease (GERD) can develop during childhood. Pathologic GERD is the occurrence of reflux with associated complications that can include failure to thrive, aspiration, laryngospasm, and esophagitis. Neurologically impaired children show higher rates of GERD than neurologically normal children and may require treatment for GERD at the time of placement of a feeding tubeFundoplication is the surgical treatment of GERD and consists of a partial (less than 360 degrees) or complete wrap or plication (i.e. folding) of the fundus of the stomach around the esophagus. This surgery can be performed using either traditional open techniques or minimally invasive techniques. At present many different fundoplication techniques are used: patients are selected for surgery only if their medical treatment failed and if they show esophagitis and stricture or Barrett esophagus. Other indications to surgery are pulmonary symptoms, specifically asthma with persistent symptoms and reflux despite medical management and recurrent pneumonia associated with GER. At present there is not the gold standard technique but in the different cases reported in literature it is possible to identify some elements that may represent a "gold standard" for any type of fundoplication: crural closure, well mobilized esophagus, floppy wrap, and the use of non-absorbable sutures. The purpose of this review is to explain each technique from a strictly technical point of view and to report the clinical and surgical outcomes in the pediatric population.

\section{9}

\section{Gastroenterology \& Nutrition}

Monitoring of Vitamin D Levels in Patients with Cystic Fibrosis in Republic of Macedonia

Tatjana Jakovska ${ }^{1}$

${ }^{T}$ Department of metabolism, University Children's Hospital, Macedonia

Vitamin D is necessary for maintaining the bone health, but vitamin D deficiency is being recognized in patients with $\mathrm{CF}$.

Aim

To assess the plasmatic levels of 25OHD in patients with $\mathrm{CF}$ who regular visit the $\mathrm{CF}$ center at the University Pediatric Clinic in Skopje, Macedonia despite the daily supplementation of 1000 IU vitamin $\mathrm{D}$ and to measure the effectiveness of higher dose oral vitamin D supplementation(2000 IE).

\section{Methods}

Serum concentrations of 25OHD were measured in $80 \mathrm{CF}$ patients from $\mathrm{CF}$ center at the University Pediatric Clinic in Skopje, R.Macedonia. 25OHD levels were determined by RIA method. The measurements were held both before changing vitamin D dosage and after at least one year of follow-up. CF patients used to receive $1000 \mathrm{IU}$ vitamin D daily. The protocol was revised to include $2000 \mathrm{IU}$ vitamin D daily dose.

\section{Results}

Vitamin D deficiency was observed at baseline in $26,25 \%$ of CF patients, from them $85 \%$ were pancreatic insufficient. The mean serum concentration of vitamin $\mathrm{D}$ at baseline was $22,7 \mathrm{ng} / \mathrm{ml}$ compared to $25,7 \mathrm{ng} / \mathrm{ml}$ at the end of follow-up (p0, 1). There was no significant difference between the protocols in vitamin D levels. The number of CF patients with deficiency using $2000 \mathrm{IU}$ decline on half, but still mean values remains low $(10,4 \mathrm{ng} / \mathrm{ml}$ versus $10.9 \mathrm{ng} / \mathrm{ml})$.

\section{Conclusion}

Despite supplementation with recommended doses of vitamin D, the study confirms levels of $25 \mathrm{OHD}$ bellow $30 \mathrm{ng} / \mathrm{ml}$ in many CF patients. Our conclusion is that these patients have need of higher doses of vitamin $\mathrm{D}$ per day and frequent monitoring of $25 \mathrm{OHD}$ levels.

\section{6}

\section{Gastroenterology \& Nutrition}

A Less Common Cause of Recurrent Abdominal Pain and Gastrointestinal Bleeding - Look for Clues

Raluca Maria Vlad $^{1,2}$, Irina Dijmarescu ${ }^{l}$, Virginia Sobek ${ }^{l}$, Dumitru Oraseanu $^{1,2}$, Daniela Pacurar ${ }^{1,2}$

${ }^{I}$ Department of Pediatrics, "Grigore Alexandrescu” Emergency Children's Hospital, Romania

${ }^{2}$ Department of Pediatrics, "Carol Davila" University of Medicine and Pharmacy, Romania 


\section{Background and objectives}

Recurrent abdominal pain is a common complaint in children, in many cases the symptoms resulting in a functional diagnosis. When gastrointestinal bleeding is associated, a red flag is raised. Complex imagistic procedures are often required to set the diagnosis.

\section{Method and results (case presentation)}

The authors present the case of a 7 years old boy with recurrent abdominal pain with onset at 2 years of age. In his medical history, he had prior presentations with gastrointestinal (GI) bleeding (rectal bleeding at 2 and hematochezia with severe anemia at 5).

The clinical examination revealed hyperpigmented spots on his lips and oral mucosa. At the time of first presentation in the Department of Pediatrics in "Grigore Alexandrescu" Emergency Children's Hospital, he associated bilious vomiting and diffuse abdominal pain, with normal stools. The blood tests showed hypochromic, microcytic anemia with low iron levels. The colonoscopy found a rectal polyp with no signs of bleeding and no specific findings on histology.

Two months later he was admitted again with anemia. Upper and lower GI endoscopy again revealed no signs of bleeding. He underwent a capsule endoscopy that found a jejunal polyp. MRI enterography set the diagnosis of intussusception on proximal jejunum. Laparotomy was required and found four intussuscepted areas (jejunal and ileal), secondary to intestinal polyps. The pathology confirmed the diagnosis of Peutz Jeghers syndrome.

\section{Conclusion}

Complete clinical examination remains an essential part of a swift diagnosis. Less common causes of recurrent abdominal pain might be discreetly indicated by easily accessible signs.

\section{8}

\section{Gastroenterology \& Nutrition}

Epidemiologic Features of Chronic Viral Hepatitis B in Romania The Experience of a Pediatrics Department

Irina Dijmarescu ${ }^{l}$, Raluca Maria Vlad ${ }^{1,2}$, Alexandra Coroleuca ${ }^{l}$, Dumitru Oraseanu $^{1,2}$, Daniela Pacurar ${ }^{1,2}$

${ }^{I}$ Department of Pediatrics, "Grigore Alexandrescu" Emergency Children's Hospital, Romania

${ }^{2}$ Department of Pediatrics, "Carol Davila" University of Medicine and Pharmacy, Romania

\section{Background}

Chronic viral hepatitis B is still a public health issue worldwide, although effective prophylaxis is available nowadays. Infection rate remains high in Romania; complete data is unavailable because a national reporting system is not implemented.

\section{Objective}

The authors aimed to analyze the epidemiology of viral hepatitis B infection in children admitted to the Pediatrics Department of "Grigore Alexandrescu" Children's Hospital.

\section{Methods}

The study included 448 children with chronic viral hepatitis B. Epidemiological data was collected for all. Each patient underwent laboratory tests: transaminases, HBs and HBe antigens, viral load.

\section{Results}

Twenty percent of the patients were also positive for hepatitis D virus, and $1.6 \%$ for hepatitis $\mathrm{C}$ virus. Regarding the strain of hepatitis B virus, $74 \%$ were wild-type viral infections, while $26 \%$ were mutants. Sixty-seven percent of the patients had active viral hepatitis (high viral load and high transaminases); $15 \%$ were in an immune tolerant state (high viral load, but normal transaminases). Nine percent were healthy carriers (negative viral load and normal transaminases). The rest of $9 \%$ had hepatitis D virus, with negative viral B load, but high transaminases. In cases of negative $\mathrm{HBe}$ antigen viral infections (mutants), $55 \%$ had active viral hepatitis and $22 \%$ were in an immune tolerant state. The rest of $23 \%$ had hepatitis D virus infection, with negative viral B load, but high transaminases. Out of the wild-type hepatitis B viral infection, $13 \%$ patients were in an immune tolerance state, while $70 \%$ presented with active disease. The rest of $5 \%$ had negative viral load, but high transaminases, and $12 \%$ were healthy carriers.

\section{Conclusion}

Epidemiological assessment of pediatric patients with chronic viral hepatitis is necessary in Romania, due to lack of consistent data. Mutant viral strains were identified in a high proportion of patients. Most of the patients have active viral hepatitis and will require treatment.

468

\section{Gastroenterology \& Nutrition}

Hepatitis-Associated Aplastic Anemia in Children: Our 4 Cases Nita Andreia Florina ${ }^{1,2}$, Coroleuca Alexandra ${ }^{1,2}$, Dijmarescu Irina ${ }^{1}$, Oraseanu Dumitru $^{I, 2}$, Pacurar Daniela ${ }^{1,2}$

${ }^{1}$ Pediatrics, "Grigore Alexandrescu" Emergency Children's Hospital, Romania ${ }^{2}$ General Medicine Faculty, "Carol Davila" University of Medicine and Pharmacy, Romania

\section{Background}

Literature reports just a few cases of Hepatitis- associated aplastic anemia (HAAA) -approximately 200 cases until 1975, with a higher prevalence in adolescent boys. Autoimmunity is thought to be the underlying mechanism, but due to scarce prevalence, little is known.

\section{Objective}

To follow and describe cases with autoimmune hepatitis (AIH) and autoimmune associated disorders (AAD), respectively aplastic anemia.

Methods

We conducted a prospective longitudinal study during 1st of January 20101st of January 2017 in the Paediatric Department of "Grigore Alexandrescu" Children's Hospital in Bucharest. We included 11 children with AIH-AAD, out of which were 4 cases with aplastic anemia. Diagnosis was established after ruling out viral, metabolic and toxic etiology.

\section{Results}

Three of the 4 cases were diagnosed as unclassified hepatitis with negative autoimmune antibodies. The forth case had Wilson disease (WD). HAAA cases were teenagers, 3 boys and a girl aged 16, 14, 14 and 13 years. All of them had been diagnosed with medullary aplasia at 3 to 4 months after an attack of acute hepatitis, with levels of transaminases within the range of 1000 to 3500 , with the exception of WD case, with values less than $600 \mathrm{U} / \mathrm{L}$.

We did not perform hepatic biopsy in none of the patients, because at the onset we considered acute hepatitis, probably viral with unidentified infectious agent. The remission of hepatic cytolysis occurred within the first month, so we did not find it necessary afterwards. Two patients received supportive treatment and one immunosuppressive therapy. For 3 cases the outcome was unfavourable, with deceases in short time due to severe pancytopenia. The only survivor in the group was the girl, with complete recovery at 6 years follow-up.

\section{Conclusions}

Children with seronegative AIH should be monitored at least 6 months in order to catch the bone marrow failure in due time.

\section{5}

\section{Gastroenterology \& Nutrition}

Status of vitamin $D$ in children and adolescents with celiac disease

Leonid Klimov ${ }^{I}$, Irina Zakharova ${ }^{2}$, Lyudmila Abramskaya ${ }^{I}$, Victoria Kuryaninova $^{1}$, Marina Stoyan ${ }^{1}$, Lyubov Kochneva ${ }^{1}, \overline{\text { Dmitriy }}$ Bobrishev $^{I}$, Georgy Anisimov ${ }^{3}$

${ }^{1}$ Pediatrics, Stavropol State Medical University, Russia

${ }^{2}$ Pediatrics, Russian Medical Academy of Continuing Postgraduate Education, Russia

${ }^{3}$ Technology of milk, North-Caucasian Federal University, Russia 


\section{Objective}

Was to analyze the status of vitamin D (VD) sufficiency in children and adolescents in the acute period of celiac disease (CD) and keeping a gluten-free diet (GFD).

\section{Methods}

Seventy-seven children with CD were examined: 29 (37.7\%) boys and $48(62.3 \%)$ girls aged 1 to 18 years, who were divided into three groups. The 1 included $22(28.6 \%)$ children examined in the acute period of CD, the 2 included $37(48.0 \%)$ patients keeping a GFD, and the 3 included 18 $(23.4 \%)$ diet non-compliant patients. The control group included 14 healthy children.

\section{Results}

The status of VD sufficiency in children of the control group was within the normal range. The levels of $25(\mathrm{OH}) \mathrm{D}$ in the patients with acute CD were 2.1 times lower (p0.001) than in the healthy children. The content of calcidiol in the GFD-compliant children was 2.0 times lower (p0.001), and in the non-compliant patients 2.4 times lower (p0.001) than in the control group.

\section{5(OH)D levels in children with CD}

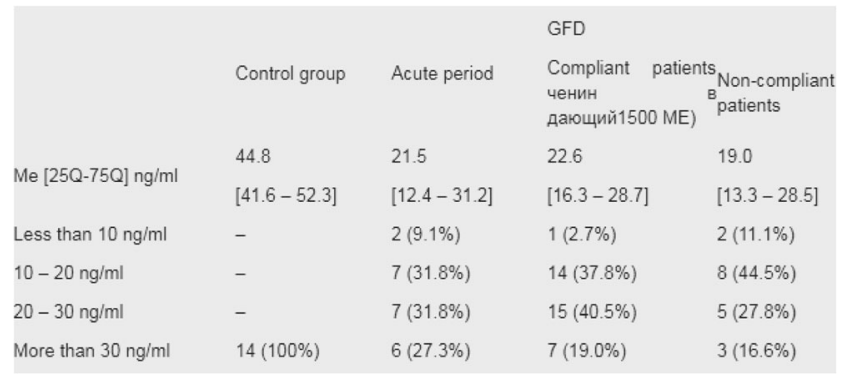

\section{Conclusions}

The study of 25(OH)D levels in blood serum indicated a low VD sufficiency in children and adolescents with $\mathrm{CD}$. A tendency to the parameters improvement in the group of patients keeping a strict GFD was observed; however, even these patients failed to reach the normal calcidiol levels without additional nutritional supplementation. It is evident that children and adolescents with celiac disease need to be provided with cholecalciferol drugs all year round to significantly improve their status of VD sufficiency.

\section{9}

\section{Gastroenterology \& Nutrition}

Efficacy of Zinc Supplementation in Reducing the Severity of Childhood Asthma Exacerbation

Sanguansak Rerksuppaphol ${ }^{l}$

${ }^{T}$ Department of Pediatrics, Srinakharinwirot University, Thailand

\section{Background}

Asthma is the most common chronic disorder of the airways in children. Airway inflammation is considered as one of the pathogenesis and pathophysiology of asthma. Zinc is an immunoregulatory agent with antioxidant, anti-inflammatory and anti-apoptotic properties. Zinc deficiency has demonstrated an association with the risk of asthma.

\section{Objective}

This study aimed to evaluate the efficacy of zinc supplementation in reducing the severity of childhood asthma exacerbation.

Methods

Forty-two children with asthma exacerbation who admitted to the hospital were randomized to receive either zinc bis-glycinate $(30 \mathrm{mg}$ elemental zinc/day) or a placebo in adjuvant to the standard treatment. The Pediatric
Respiratory Assessment Measure (PRAM) was used to measure the asthma severity. The primary outcome was a change in asthma severity from the baseline to the end of study. The secondary outcomes were change in serum zinc levels from the baseline to the end of study and prevalence of zinc deficiency.

\section{Results}

Baseline asthma severity scores did not show a significant difference between groups. However, the PRAM score in the zinc group showed a more rapid decrease compared to the control group at the 24-hour $(2.2 \pm 1.3$ vs $1.2 \pm 1.3 ;$ p-value $=0.015)$ and 48 -hour $(3.4 \pm 2.0$ vs $2.2 \pm 1.8 ; \mathrm{p}$-value $=0.042$ ) intervals. At admission, overall mean serum zinc level was $63.8 \mu \mathrm{g} / \mathrm{dL}$ and $57.1 \%$ of children had zinc deficiency with no difference in prevalence between groups. PRAM scores did not differ between children with low and normal zinc status. Compared to baseline, zinc levels increased by $32.3 \mu \mathrm{g} / \mathrm{dL}$ and $14.2 \mu \mathrm{g} / \mathrm{dL}$ in the zinc and control groups, respectively. However, zinc levels at the end and length of hospital stay were not significantly different between the groups.

\section{Conclusion}

Zinc supplementation as the adjuvant therapy to the standard treatment during asthma exacerbation resulted in rapid lessening of severity.

\section{2}

\section{Gastroenterology \& Nutrition}

Comparative analysis of open echinococcectomy vs. pair technique in children - 16 years experience

Georgi Ivanov ${ }^{I}$, Anton Tonev ${ }^{2}$, Nikola Kolev $^{2}$, Aleksandar Zlatarov ${ }^{2}$, Georgi Todorov, Chavdar Bachvarov ${ }^{3}$, Plamen Kokenski ${ }^{1}$, Dilqn Petrov $^{4}$, Valentin Ignatov ${ }^{2}$, Krasimir Ivanov ${ }^{2}$

1 "Department of General surgery-pediatric surgery", University Hospital "St. Marina" Varna, Bulgaria, Bulgaria

2 "Department of General surgery", University Hospital "St. Marina" Varna, Bulgaria, Bulgaria

3 “Department of Radiology", University Hospital "St. Marina” Varna, Bulgaria, Bulgaria

4 "Department of General surgery", University Hospital "St. Marina" Varna, Bulgaria, Bulgaria

\section{BACKGROUND}

Hydatidosis is a major problem in pediatric practice that can cause significant morbidity and mortality. The liver is the organ affected most frequently.

AIM

This study was carried out to compare the results and efficiency of miniinvasive and operative treatment of hydatid liver disease in pediatric patients.

\section{MATHERIAL AND METHODS}

Comparative analysis of surgical and percutaneous treatment of children with hepatic hydatid cysts is presented. Data was collected from the records of 118 patients with hydatidosis from 2001 to 2016.

\section{RESULTS}

Minor complications were urticaria and fever in 6 patients with PAIR method and inflammation of the surgical wound in 5 patients. Major complications were infection of the cyst cavity in a patient and development of biliary fistula in 3 patients underwent operative surgery. No site recurrences were observed in both of groups, but in four patients we inspected progression of the disease. No mortality, abdominal dissemination, or tract seeding occurred.

\section{CONCLUSION}

Surgery should no longer be regarded as the first choice treatment. PAIR can effectively replace surgical techniques in treatment of liver hydatid disease and may be imposed as a first method of choice. 
602

\section{Gastroenterology \& Nutrition}

Clinical Exome Sequencing of Five Year Old Girl with Hepatosplenomegaly, Cholestasis, and Hypercholesterolemia Natasa Nikcevska ${ }^{I}$, Sonja Petrovska ${ }^{1}$, Mirko Spirovski ${ }^{2}$, Sanja Mehandziska $^{2,3}$, Goran Kungulovski ${ }^{2,3}$, Zan Mitrev ${ }^{2}$

${ }^{1}$ pediatrics, Zan Mitrev Clinic, Macedonia

${ }^{2}$ genetics and personalised medicine, Zan Mitrev Clinic, Macedonia

${ }^{3}$ genetics, Bioengineering LLC, Skopje, Macedonia

\section{BACKGROUND}

Progressive familial intrahepatic cholestasis type 3 is an autosomal recessive condition characterised by hepatocellular phospholipid export deficiency, impaired secretion, caused by mutations in the ABCB4 gene. Lack of phospholipids produces unstable micelles with toxic effect on the bile ducts, leading to cholestasis.

\section{OBJECTIVE}

5 year old girl referred for pediatric checkup and further genetic examination due to hepatosplenomegaly, cholestasis presented as elevated GGT and hypercholesterolemia. First symptom was anemia, at 7 months of age. She was hospitalized for elevated hepatic transaminases, and hypercholesterolemia. Thorough evaluation in finding the etiology was done. Major metabolic diseases, extrahepatic biliary obstruction and infectious pathology were all excluded. Later patient manifested cholestasis with liver and spleen enlargement, telangiectasia, poor weight gain, pruritus and signs of rickets. Treatment was started with ursodeoxycholic acid.At first glycogen storage disease type 6 was suspected; later dismissed by Sanger sequencing of the PYGL gene. Parents refused liver biopsy, thus clinical exome sequencing was recommended.

\section{METHODS}

Clinical exome sequencing of 4811 clinically relevant genes was performed on a NextSeq Illumina instrument. Upon quality control, DNA sequences were mapped to hg19, and a total number of 7790 highconfidence genetic variants were called.

\section{RESULTS}

Two candidate pathogenic variants c.2165GC; p.Gly722Ala; and c.833GT; p.Arg278Met in ABCB4 were identified. Genetic variant c. $2165 \mathrm{GC}$ has been reported in the Clinvar database, while to the best of our knowledge, c.833GT is not reported and was discovered for the very first time. Both meet several pathogenicity criteria. Pathogenic mutations in ABCB4 are associated with PFIC type 3 (PFIC3; OMIM: 171060)

\section{CONCLUSION}

We conclude that patients who present with cholestasis, in their differential diagnosis should be considered for PFIC, even though cholestasis is not the dominant sign. Here we show that compound heterozygosity of one novel and one known ABCB4 mutation could lead to PFIC3.

607

\section{Gastroenterology \& Nutrition}

Different Bowel Habit Subtypes of Irritable Bowel Syndrome May Response Different to Vitamin D Supplementation Regarding Inflammatory Cytokines, Oxidative Stress Biomarkers and Clinical Symptoms

Amir Abbasnezhad ${ }^{1}$, Razieh Choghakhori ${ }^{1}$, Reza Amani ${ }^{2}$

${ }^{T}$ Department of Nutrition, Nutritional Health Research Center, Lorestan University of Medical Sciences, Khorramabad, Iran

${ }^{2}$ Department of Nutrition, Diabetes Research Center, Health Research Institute, Ahvaz.Jundishapur University of Medical Sciences, Ahvaz,., Iran

\section{Background}

Considering the variations in clinical characteristics and pathophysiological mechanisms among irritable bowel syndrome (IBS) subtypes, it is a well-established fact that the response to treatments are different.

\section{Objective}

We evaluated the effect of vitamin D on inflammatory cytokines, oxidative stress biomarkers in IBS. Furthermore, we analyzed the effect of vitamin D in different subtypes of IBS.

Methods

A total of ninety IBS patients, as defined by the Rome III criteria, participated in this double blind, randomized, placebo-controlled 6-month intervention. Participants were randomly categorized to receive either 50,000 IU vitamin $\mathrm{D}_{3}$ or a placebo fortnightly. Patients were further sub-classified as diarrhea predominant (IBS-D), constipation-predominant (IBS-C), and alternating bowel habits (IBS-A). Gastrointestinal (GI) symptoms, biochemical factors (such as IL-17, IL-10, TNF $\alpha$, malondialdehyde (MDA) and total antioxidant capacity (TAC)) were assessed.

Results

Following vitamin D supplementation, the serum IL-17 and MDA levels significantly decreased, TAC and IL-10 levels significantly increased compared to the placebo group. When comparing different subtypes, the serum TNF $\alpha$ and IL-17 levels significantly $\left(\begin{array}{ll}P & 0.05\end{array}\right)$ decreased in IBS-D, whereas IL-10, MDA and TAC significantly $(P 0.05)$ changed in all the subtypes. Following vitamin D supplementation, abdominal pain, abdominal distention, flatulence and overall GI symptoms were significantly $(P$ 0.05) decreased in IBS-D patients with no significant changes in other subtypes.

\section{Conclusion}

Vitamin D has anti-inflammatory and antioxidant effects in patients with IBS. Vitamin D supplementation could ameliorate the GI symptoms in IBS-D. Thus, the present study suggests the beneficial effect of vitamin D in IBS-D.

612

\section{Gastroenterology \& Nutrition}

Vitamin D Deficiency in Patients with Irritable Bowel Syndrome: Association with Clinical Symptoms, Quality of Life and Inflammatory Cytokines

Amir Abbasnezhad ${ }^{1}$, Razieh Choghakhori ${ }^{1}$, Amin Hasanvand ${ }^{2}$, Esmaeil Yousefi Rad', Somayeh Saboori

${ }^{I}$ Department of Nutrition, Nutritional Health Research Center, Lorestan University of Medical Sciences, Khorramabad, Iran

${ }^{2}$ Department of Pharmacology and Toxicology, Faculty of Pharmacy, Lorestan University of Medical Sciences, Khorramabad,, Iran

\section{Background}

Vitamin D deficiency (VDD) is highly prevalent in gastrointestinal (GI) disorders and might play an important role in the development of these disorders.

\section{Objective}

We assessed VDD and also the possible correlation of the serum vitamin D with clinical symptoms, quality of life (QoL) and inflammatory cytokines in IBS patients.

\section{Methods}

A total of 90 IBS patients diagnosed according to Rome III criteria (all subtypes) were recruited from the tertiary referral Hospital of the University. Also 90 sex and age matched HCs without GI symptoms took part in the study. Blood was drawn for measurement of the serum $25(\mathrm{OH}) \mathrm{D}_{3}$ levels and cytokines interleukin (IL)-10, IL-17 and tumor necrosis factor (TNF) $\alpha$. Furthermore, IBS symptoms severity score (IBSSS), IBS quality of life (IBS-QOL) and the severity of GI symptoms were evaluated.

\section{Results}

The serum $25(\mathrm{OH}) \mathrm{D}_{3}$ was significantly ( $\mathrm{P}$ 0.05) lower in IBS patients. When comparing different bowel habit subtypes with $\mathrm{HCs}$, the serum $25(\mathrm{OH}) \mathrm{D}_{3}$ was significantly ( $\mathrm{P}$ 0.05) lower in 
diarrhea-predominant IBS (IBS-D). Furthermore, the lower serum $25(\mathrm{OH}) \mathrm{D}_{3}$ was significantly $(\mathrm{P} 0.05)$ associated with higher proinflammatory cytokines (TNF $\alpha$ and IL-17) and lower antiinflammatory cytokines (IL-10). The lower serum $25(\mathrm{OH}) \mathrm{D}_{3}$ was significantly (P 0.05) associated with higher severity of GI symptoms and IBSSS, whereas a significant positive association was found between the lower serum $25(\mathrm{OH}) \mathrm{D}_{3}$ and IBS-QoL.

\section{Conclusions}

Our study revealed that VDD is highly prevalent in patients with IBS; especially, in IBS-D. VDD is correlated with the higher severity of the IBS symptoms and lower QoL.

\section{IBS-QoL}
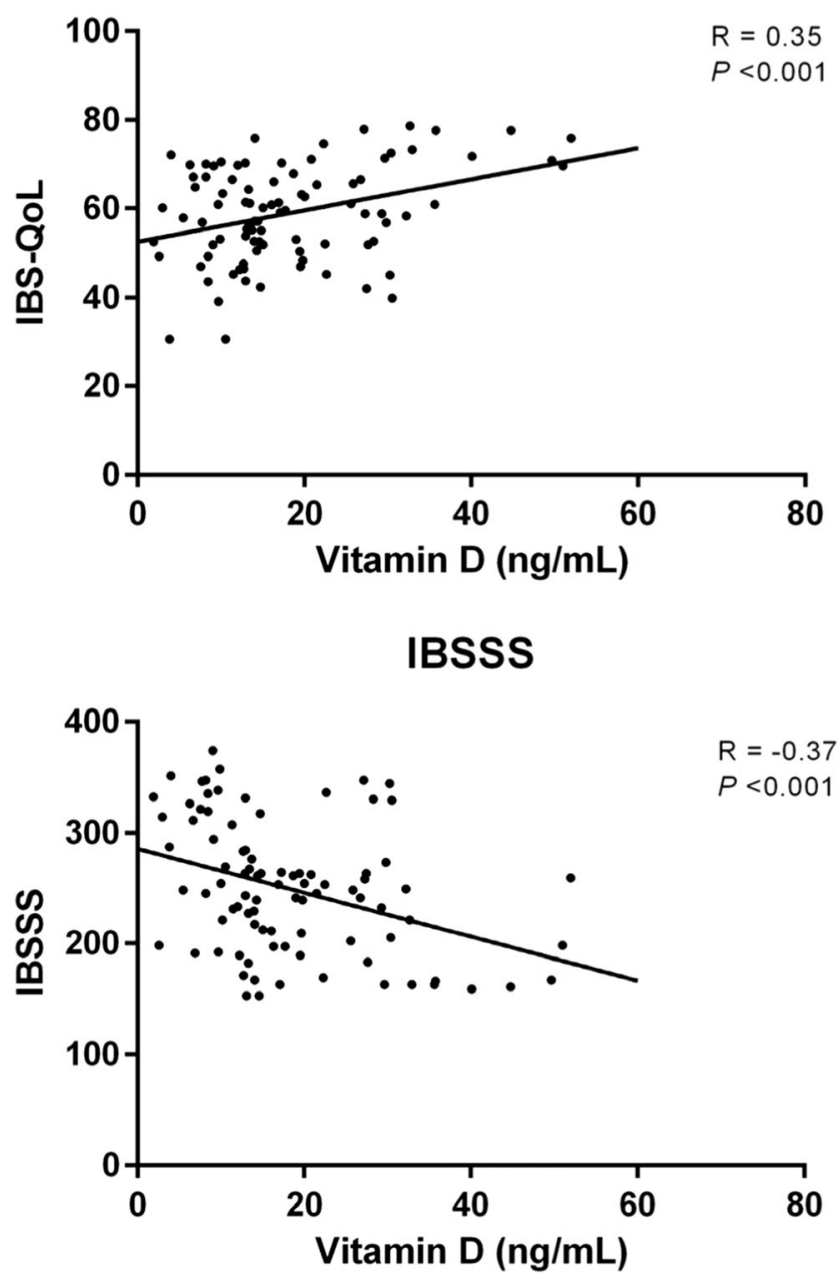

0.05 were considered statistically significant. IBS, irritable bowel syndrome; IBSSS, IBS symptoms severity score; IBS-QoL, IBS quality of life." width $=$ " 317 " height $=$ " $485 " /$

\section{8}

\section{Gastroenterology \& Nutrition}

Vitamin D - do we know enough?

Bianca Elena Popovici ${ }^{1}$, Maria Mitrica ${ }^{1}$, Flaviu Moldovan ${ }^{3}$, Luciana Petrescu ${ }^{2}$ ${ }^{T}$ Pediatrics, Transilvania University, Faculty of Medicine, Romania

${ }^{2}$ Intensive Care Unit, Children Hospital, Romania

${ }^{3}$ Pediatrics, University of Medicine and Farmacy, Romania

\section{Introduction}

Vitamin D is necessary for health in children as well in adults.

Aim of the study

To evaluate the level of knowledge of parents regarding Vitamin D and its importance in child health.

\section{Material and method}

We had performed a prospective study including 200 subjects, based on questionnaire: the data were collected and processed.

\section{Results}

The subjects came from urban area (76\%) and rural area (24\%); most of them had medium education - high school $(88 \% 0$, faculty $(10 \% 0$ and primary school (2\%). The source of medical information was: internet (58\%), knowledge from school $(13.5 \%)$, medical staff - familiy doctor and pediatrician (28.5\%). Regarding the role of Vitamin D, $65 \%$ of subjects knew the connection with calcium and phosphorus absorbtion and bone integrity and $35 \%$ had no informations. $58.5 \%$ of subjects knew the role of sun light exposure in Vitamin D synthesis and $52.8 \%$ could enumerate few main diet sources of Vitamin D. The informations were pour regarding the dosis, period of year, and lenght of administration of Vitamin D. data about ricket were also pour $950 \%$ of subjects knew about it and connection with Vitamin D deficiency, from those, $68 \%$ knew signs of ricket); $48 \%$ of subjects knew about intoxication sings with Vitamin D and the correct attitude.

Conclusions

The knowledge about Vitamin D were satisfactory. The knowledge about ricket were pour. the administration of Vitamin $\mathrm{D}$ is accepted by parents as "something that must be done" but with no connection with ricket nor with other effects on body. The main source of information was media, internet, and discussions with other parents on forum; the parental decisions were influenced by other parents advices. Medical policy sholud focus on better information and communication with parents.

652

\section{Gastroenterology \& Nutrition}

The impact of mothers' LEPR rs1137101 gene polymorphisms on neonatal obesity risk

Oana Marginean ${ }^{1}$, Claudiu Marginean ${ }^{2}$, Mihaela Iancu ${ }^{3}$, Lorena Elena $\overline{\text { Melit }}^{\prime}$, Maria Oana Marginean ${ }^{I}$, Claudia Banescu ${ }^{4}$

${ }^{1}$ Pediatrics, University of Medicine and Pharmacy Tirgu Mures, Romania

${ }^{2}$ Obstetrics and Gynecology, University of Medicine and Pharmacy Tirgu Mures, Romania

${ }^{3}$ Department of Medical Informatics and Biostatistics, University of Medicine and Pharmacy Cluj Napoca, Romania

${ }^{4}$ Genetics, University of Medicine and Pharmacy Tirgu Mures, Romania

\section{Introduction}

LEPR rs1137101 (Gln223Arg) gene polymorphism is one of the most frequent polymorphisms associated with obesity. Therefore, it is important to assess these genetic factors, anthropometric parameters and bioimpedance results in order to evaluate the nutritional status.

The aim of this study was to establish the role of mothers' LEPR rs 1137101 gene polymorphisms on newborns' parameters, especially birth weight, and therefore establishing the impact on the neonatal obesity risk.

Material and methods

We performed a cross-sectional study on 215 mothers-newborns couples in a Tertiary Hospital from Romania. Both mothers and newborns were assessed anthropometrically, clinically and regarding the LEPR rs1137101 gene polymorphisms.

Results

We noticed that the mothers' $L E P R$ rs 1137101 gene polymorphisms influenced the newborns' weight ( $p=0.0078)$ and BMI $(p=0.004)$. Also, newborns that carry AG + GG genotype whose mothers had AA genotype, presented higher birth weight and BMI $(p=0.0054, p=0.038)$ in comparison with those carrying AA genotype. In newborns and mothers that carry the variant allele, an interaction between LEPR rs 1137101 gene polymorphisms and MUAC was encountered $(p=0.078)$. In addition, 
newborns carrying AG + GG genotype of LEPR rs1137101 gene whose mothers had an increased FMI (fat mass index), presented a significantly higher BMI $((p=0.0028)$.

\section{Conclusions}

The LEPR rs1137101 gene polymorphisms have a significant influence on newborns' birth weight and BMI depending on the presence or absence of variant allele in mothers. Maternal FMI presented a significant positive effect on newborns' BMI.

We can conclude that mothers' LEPR rs1137101 gene polymorphisms present an impact on newborns' birth weight and BMI, therefore being involved in the newborns' risk for developing obesity.

This research was partially supported by the Collective Research Grants of the University of Medicine and Pharmacy Tîrgu Mureș, Romania ("The role of mother's genetic determinism in child's obesity correlated with bioimpedance and anthropometric parameters" no.275/4/ 11.01.2017)

\section{7}

\section{Gastroenterology \& Nutrition}

Serotonin Blood Level in Children with Overlapping of Irritable Bowel Syndrom and Functional Dyspesia

Zimnytska Tatiana ${ }^{l}$, Rakovska Ludmila ${ }^{I}$, Panko Natalia $^{l}$, Golovko Tatiana ${ }^{I}$

${ }^{T}$ Pediatrics, VN Karazin Kharkiv National University, Ukraine

\section{Background}

The epidemiological studies of the prevalence of functional dyspepsia (FD) and irritable bowel syndrome (IBS) showed a wide range (13-87\%) of the overlap between them (Fujiwara, 2014). The main clinical symptom in children with FD as well as IBS is abdominal pain, which has different characteristics, but similar origins. Both of these functional gastrointestinal disorders are largely dependent on the condition of the gut motility and visceral hypersensitivity on a background of neurohormonal dysregulation. Serotonin plays an important role in these processes taking part in nociceptive transmission and regulation of a pain impulse (J Talley Nicholas, 2013).

\section{Objective}

Evaluation of serotonin blood level in children with overlapping of IBS and FD.

\section{Methods}

The blood concentration of serotonin was measured by the fluorometric assay in 43 children, aged from 10 to 15 years old with IBS (the main group) and 10 healthy individuals of the same age (the control group). The main group was divided into two subgroups: 23 children with overlapping IBS and FD and 20 children with IBS only. The diagnosis was established clinically according to the Rome IV Diagnostic Criteria (2016). The accuracy of the diagnosis was confirmed by the laboratory tests and low and upper endoscopies.

\section{Results}

The mean value of the serotonin blood level in children with overlapping IBS and FD was $3.56 \pm 0.02 \mathrm{mkmol} / \mathrm{L}$ and it markedly exceeds (p0,01) this parameter in the control group $(1.45 \pm 0.15 \mathrm{mkmol} / \mathrm{L})$. It was also higher $(\mathrm{p} 0,05)$ than the same one in the patients with IBS only $(2.62 \pm 0.26 \mathrm{mkmol} / \mathrm{L})$

Conclusions

We have found the higher blood level of serotonin in children with overlapping of IBS and FD compared with the ones with IBS only. It should be taken into account for management of such patients.

694

\section{Gastroenterology \& Nutrition}

Cystic fibrosis and glycogen storage disease: A case report of a rare association

Aysel Yuce ${ }^{1}$, Meryem Seda Boyraz ${ }^{I}$, Gokcen Tugcu ${ }^{l}$, Pinar Kahyaoglu ${ }^{l}$, Nural Kiper ${ }^{I}$, Inci Nur Saltik Temizel ${ }^{1}$, Ugur Ozcelik

${ }^{I}$ Department of Pediatrics, Hacettepe University, Faculty of Medicine, Turkey

\section{Background and aims}

Glycogen storage diseases (GSD) are inborn error of the glucose and glycogen metabolism resulting from mutations in genes responsible for glycogen synthesis, degradation or regulation. Abnormal glycogen is stored in various tissues, especially liver and muscle. Cystic fibrosis (CF) is caused by the mutations in the CFTR gene. These mutations cause thick and viscous secretions in all exocrine tissues. The co-existence of two inherited liver diseases in the same patient has not been reported before. We reported the first case of cystic fibrosis with glycogen storage disease on a 17 month-old boy.

\section{Methods}

Case report

\section{Results}

A 17 month-old boy who was diagnosed as cystic fibrosis during neonatal screening (serum immunoreactive trypsinogen was positive. Sweat chloride testing was abnormal $(104 \mathrm{mEq} / \mathrm{L})$. CF mutation analysis revealed p.E92K GA homozygosity. The parents are second degree cousins. He was referred to our hospital due to hepatomegaly and elevated transaminases levels. Since he had massive hepatomegaly and a history of older brother who died of GSD type 3, GSDs were suspected. Laboratory findings were also suggestive for GSD type 3 such as high serum levels of triglycerides and creatine kinase. Liver biopsy was performed and histopathological findings were consistent with GSD. Hepatic glycogen content was high ( $9 \mathrm{~g} / 100 \mathrm{~g}$ liver tissue; normal 5-7 g/100 g liver tissue). Conclusion

This is the first report of co-existence of CF and GSD in a child. Cystic fibrosis and GSD are rare inherited diseases and co-existence is unusual. However it may not be surprising in countries where parental consanguinity rate is high.

\section{7}

\section{Gastroenterology \& Nutrition}

Exclusive Enteral Nutrition and Remission in Pediatric Crohn's Disease: How Deep is Deep Enough?

Lacramioara Eliza Pop $^{1,2}$, Otilia Fufezan ${ }^{3}$, Diana Hopulele ${ }^{1,2}$, DanielaElena Serban ${ }^{1,2}$

${ }^{1}$ Second Department of Pediatrics, Emergency Hospital for Children, Romania ${ }^{2}$ Second Department of Pediatrics, "Iuliu Hatieganu" University of Medicine and Pharmacy, Romania

${ }^{3}$ Third Department of Pediatrics, Emergency Hospital for Children, Romania

\section{BACKGROUND}

Deep remission [clinical remission (CR) associated with mucosal healing $(\mathrm{MH})$ ] represents the treatment target in Crohn's disease (CD). Exclusive enteral nutrition (EEN) is recommended as first-choice agent in pediatric $\mathrm{CD}$, being effective in inducing deep remission (DR). However, its efficacy on transmural inflammation has been rarely investigated.

\section{OBJECTIVE}

We aimed to assess the efficacy of EEN on transmural healing (TH) in CD and identify predictors for remission.

\section{METHODS}

All CD patients from our tertiary centre database (2006-2017) were reviewed and included if EEN was used for induction of remission, as first-line therapy, over 6-12 weeks. All parameters were evaluated at baseline and at the end of induction period. The following definitions of remission were used: CR (wPCDAI 12.5 points), biological remission (BR, normal C-reactive protein), $\mathrm{MH}$ (normal fecal calprotectin \pm normal ileocolonoscopy) and TH (normal transmural aspect on contrast ultrasonography, plus MH). STATA software version 13 was used for analysis.

\section{RESULTS}

Of the 44 newly diagnosed CD patients, 20 (mean age at diagnosis $12.32 \pm 4.3$ years, $67 \%$ male) met the inclusion criteria. $\mathrm{CR}$ was detected in 17 cases $(85 \%)$, BR in $18(90 \%), \mathrm{MH}$ in $10(50 \%)$ and $\mathrm{TH}$ in $4(20 \%)$. Of the17 patients with $\mathrm{CR}$, all achieved BR, but only $24 \% \mathrm{MH}$ and $12 \% \mathrm{TH}$ $(p=0.05)$. In multivariate analysis, of all parameters, only weight loss 5 
kilograms before diagnosis was found to negatively affect $\mathrm{MH}$ and $\mathrm{TH}$ $(\mathrm{OR}=12.99 ; 95 \% \mathrm{CI}=1.7-99.36 ; p=0.013)$.

\section{CONCLUSION}

$\mathrm{CR}$, assessed by wPCDAI, is detected in $85 \%$ of patients; however, only half of patients achieve DR and one fifth TH. Of children in CR, TH is detected in only $12 \%$. Therefore, we consider that deep remission should include $\mathrm{TH}$; otherwise young patients could be at risk of developing progressive bowel damage, like stenosis, fistula and abscesses, with possible definitive disabilities.

\section{7}

\section{Gastroenterology \& Nutrition}

Progressive Familial Intrahepatic cholestasis type3 resulting from compound heterozygosity of $\mathrm{ABCB} 4$ mutations

Natasa Nikcevskal, Sonja Petrovskal, Sanja Mehandziska ${ }^{2,3}$, Mirko Spirovski ${ }^{2}$, Goran Kungulovski ${ }^{2,3}$, Zan Mitrev ${ }^{2}$

${ }^{1}$ pediatrics, Zan Mitrev Clinic, Macedonia

${ }^{2}$ genetics and personalised medicine, Zan Mitrev Clinic, Macedonia

${ }^{3}$ genetics, Bioengineering LLC, Skopje, Macedonia

\section{BACKGROUND}

Progressive familial intrahepatic cholestasis type 3 is an autosomal recessive condition characterised by hepatocellular phospholipid export deficiency, impaired secretion, caused by mutations in the ABCB4 gene. Lack of phospholipids produces unstable micelles with toxic effect on the bile ducts, leading to cholestasis.

\section{OBJECTIVE}

5 year old girl referred for pediatric checkup and further genetic examination due to hepatosplenomegaly, cholestasis presented as elevated GGT and hypercholesterolemia. First symptom was anemia, at 7 months of age. She was hospitalized for elevated hepatic transaminases, and hypercholesterolemia. Thorough evaluation in finding the etiology was done. Major metabolic diseases, extrahepatic biliary obstruction and infectious pathology were all excluded. Later patient manifested cholestasis with liver and spleen enlargement, telangiectasia, poor weight gain, pruritus and signs of rickets. Treatment was started with ursodeoxycholic acid.At first glycogen storage disease type 6 was suspected; later dismissed by Sanger sequencing of the PYGL gene. Parents refused liver biopsy, thus clinical exome sequencing was recommended.

\section{METHODS}

Clinical exome sequencing of 4811 clinically relevant genes was performed on a NextSeq Illumina instrument. Upon quality control, DNA sequences were mapped to hg19, and a total number of 7790 high-confidence genetic variants were called.

\section{RESULTS}

Two candidate pathogenic variants c.2165GC; p.Gly722Ala; and c.833GT; p.Arg278Met in ABCB4 were identified. Genetic variant c.2165GC has been reported in the Clinvar database, while to the best of our knowledge, c.833GT is not reported and was discovered for the very first time. Both meet several pathogenicity criteria. Pathogenic mutations in $\mathrm{ABCB} 4$ are associated with PFIC type 3 (PFIC3; OMIM: 171060)

\section{CONCLUSION}

We conclude that patients who present with cholestasis, in their differential diagnosis should be considered for PFIC, even though cholestasis is not the dominant sign. Here we show that compound heterozygosity of one novel and one known ABCB4 mutation could lead to PFIC3.

\section{3}

\section{Gastroenterology \& Nutrition}

The condition of the esophagus and stomach in children with atopic dermatitis

Anastasia listopadova ${ }^{1}$, Valeria Novikova ${ }^{2}$, Olga Demchenkova ${ }^{I}, \underline{\text { Yulia }}$ Zamyatina ${ }^{1,3}$

${ }^{T}$ pediatrics, 8 th Children's Polyclinic, Russia
${ }_{2}^{2}$ pediatrics, Federal Medical Research Center Almazov, Russia ${ }_{3}^{3}$ pediatrics, North-western State Medical University named after I.I.Mechnikov, Russia

The aim is to study the characteristics of esophageal mucosa in children with atopic dermatitis. Patients and methods: We examined 30 children aged 10 to 17 years (mean age $13.4 \pm 2.9$ ) with chronic gastritis $(\mathrm{CG})$ and atopic dermatitis (AD). The comparison group consisted of 33 children suffering from CG. The main group consisted of patients with $\mathrm{AD}$ in mild to moderate disease severity. In the examined patients was observed partially localized and widespread skin lesions. Clinical condition survey was conducted on a scale SCORAD. All patients underwent standard gastroenterological examination, including fibrogastroscopy (FGS). Statistical processing of the research materials was carried out using Statistica for Windows.Results: Allergic history in children with $\mathrm{CG}$ and $\mathrm{AD}$ had significantly more often $(26.67 \% 0.00 \%$, p 0.001$)$. In $83.3 \%$ of children with atopic dermatitis revealed sensitization to cow's milk protein - in $66.6 \%$, to the egg - $79 \%$; an allergic reaction to fish in $66.6 \%$, for a chicken $-50 \%$, wheat $-36.6 \%$, oats - from $23.3 \%$ of patients. Sensitization to domestic and epidermal allergens can be found at $6.67 \%$ of children with $\mathrm{AD}$ and $\mathrm{CG}$, and allergic to tree pollen was observed in $15.6 \%$. Complaints of heartburn equally apply to the patients in both groups $(40.00 \%$ at $30.30 \%, \mathrm{p} 0.05$ ). Among comorbidities in children with $\mathrm{AD}$ were significantly more often identified the presence of asthma $(13.33 \%$ and $0 \%$, $\mathrm{p} 0.05$ ). While reflux esophagitis in children with $\mathrm{AD}$ also was diagnosed significantly more often ( $20 \%$ and $3.03 \%$, p0.05). According to FGS in patients with $\mathrm{AD}$ was significantly more often detected erosive esophagitis (26.67\% and $6.06 \%, \mathrm{p} 0.05)$, gastroesophageal reflux $(26.67 \%$ and $6.06 \%, \mathrm{p} 0.05)$ and insufficiency of the cardia (20\% and $3.03 \%, \mathrm{p} 0.05)$. Conclusion: in children with atopic dermatitis were significantly more often identified with erosive reflux esophagitis that requires study of the relationship of atopic dermatitis and disease of the esophagus.

\section{0}

Gastroenterology \& Nutrition

\section{PREDISPOSING FACTORS IN PORTAL VEIN OBSTRUCTION IN CHILDREN}

Alina Gramal, Gheorghe Popa ${ }^{1}$, Lucia Burac ${ }^{1}$, Alexandru Pirvan ${ }^{1}$, Bianca Simionescu ${ }^{I}$, Cristina Blag ${ }^{1}$, Mihaela Spirchez ${ }^{l}$, Otilia Fufezan ${ }^{2}$, Tudor L Pop ${ }^{1}$

${ }^{1}$ Pediatric Gastroenterology, 2nd Pediatric Clinic, University of Medicine and Pharmacy "Iuliu Hațieganu” Cluj-Napoca, Romania, Romania

${ }^{2}$ Radiology, 3rd Pediatric Clinic, University of Medicine and Pharmacy "Iuliu Hațieganu” Cluj-Napoca, Romania, Romania

\section{Introduction}

The portal vein thrombosis (PVT) is one of the most important causes of portal hypertension among children. The initial clinical manifestation can be represented by upper gastrointestinal bleeding, splenomegaly or ascitis. In children, the most common risk factors of thrombosis in the port system are: neonatal vein catheterization, umbilical cord infections, blood transfusions, sepsis, dehydration, coagulation abnormalities (thrombophilia, antiphospholipid syndrome).

Purpose

The aim of our study was to evaluate the risk factors for PVT in children. Methods

We have analysed retrospectively all patients who were admitted to our hospital with PVT between January 2012 and May 2017.

\section{Results}

During the last 5 years, 40 were admitted to our hospital with PVT. The mean age at the diagnosis was 7.48 . The clinical presentation was with upper gastrointestinal bleeding in 20 patients $(50 \%)$. 31 children $(77.5 \%)$ were umbilical catheterized immediately after birth. This was the most important risk factor for PVT in our patients. Sepsis in neonatal period 
were associated in other 11 cases $(27.5 \%)$. Fourteen patients were investigated for thrombophilia, 12 (70 \%) being with factor V Leiden mutation, factor II mutation, deficiency of protein $\mathrm{C}$, protein $\mathrm{S}$ or antithrombin III. Conclusion

The increasing use of umbilical catheterization in our Neonatal Intensive Care Units and especially the use of these catheters for the purpose of administering of any type of medication could be linked with the increased number of cases diagnosed with PVT. The genetic factor certainly plays an important role among these patients. Active ultrasound evaluation of children with risk factor for PVT should be encouraged for an early diagnosis and treatment

476

Genetic \& Metabolic

THE USE OF ARRAY CGH IN A COHORT OF ALBANIAN CHILDREN WITH DEVELOPMENT DELAY/INTELLECTUAL DISABILITY, CONGENITAL ANOMALIES AND DYSMORPHIC FEATURES

Anila Babameto-Laku ${ }^{l}$, Dorina Roko ${ }^{I}$, Mirela Tabaku ${ }^{l}$, Donjeta Bali $\left(\right.$ Alia $^{2}$

${ }^{T}$ Service of Medical Genetics, Pediatric Department, Faculty of Medicine, University Hospital Center "Mother Teresa", Albania

${ }^{2}$ Service of Oncohematology, Pediatric Department, Faculty of Medicine, University Hospital Center "Mother Teresa", Albania

\section{Background}

The aim of our study was to identify chromosomal imbalances by microarray comparative genomic hybridization (array CGH) in DNA samples of children with developmental delay/intellectual disability, congenital anomalies and dysmorphic features, in which karyotype results cannot be obtained. This increased resolution of microarray technology over conventional cytogenetic analysis allows for identification of chromosomal imbalances primarily because of its higher sensitivity for submicroscopic deletions and duplications.

Objective

To present the first Albanian experience of an array CGH application. The cohort included 13 children with with developmental delay/intellectual disability, congenital anomalies and dysmorphic features. The patients were newborns and children with congenital anomalies diagnosed for the time period 20142016

Methods

The cytogenetic analysis determined by a standard method of Gbanding was performed for all our patients, while array CGH was performed on genomic DNA isolated from the blood of 11 cases. For all patients, karyotype analysis has been extended to parental samples.

Results

Among the 13 patients for one male patient, standard G-banding revealed 46,XY,dup(10)(q22) karyotype and for another male patient revealed 46,XY,del (7) (q21q22.2) karyotype. Among 11 patients that resulted with normal karyotype, array CGH revealed 4 patients $(36.3 \%)$ resulted with a duplication and a deletion, 2 patients $(18 \%)$ with a microdeletion, 2 patients $(18 \%)$ with a duplication, 2 patients $(18 \%)$ with a deletion and 1 patient $(9 \%)$ with two deletions. In 3 out of the 11 patients the chromosomal aberration had been inherited from one of the healthy parents, carrier of balanced reciprocal translocation.

\section{Conclusion}

Array-CGH has improved significantly the genetic diagnosis of pediatric patients with developmental delay/intellectual disability, congenital anomalies and dysmorphic features.

The identification of the specific genetic defect in these patients clarify the diagnosis, suggest the prognosis and elucidate the risk of a disease in family members and future children.
- Keywords: congenital anomalies, deletion, duplication, intellectual disability, karyotype, microarray comparative genomic hybridization.

9

Genetic \& Metabolic

Impact of obesity and nitric oxide synthase gene polymorphism on essential hypertension at children

Svetlana Cojocari ${ }^{1}$, Nelea Mătrăgună ${ }^{1}$, Bichir-Thoreac Lilia ${ }^{1}$, Nicolae Barbacar $^{2}$

${ }_{2}^{1}$ pediatric cardiology, Cardiologycal Institute, Moldova, Republic of

${ }^{2}$ genetic, The Institute of Genetics, Physiology and Plants Protection of the Academy of Science in Moldova, Moldova, Republic of

\section{Background}

Hypertension is a multifactorial disease caused by environmental, metabolic and genetic factors, but currently it is little known about the complex interplay between these factors and blood pressure.

The aim of the study was: estimation of the relations between carrying of gene polymorphism Glu298Asp of endothelial nitric oxide synthase gene and risk of arterial hypertension at children with obesity/overweight

\section{Methods}

The investigation included 120 children with obesity/overweight at the age of $10-16(n=62 ; 51,67 \%$ - hypertensives and $n=58 ; 48,33 \%$ - normotensives). The control group included 50 children with normal tension and weight of the similar age - 26 boys and 24 girls, without family history including arterial hypertension and obesity. The protocol of the study was approved by the Medical Ethics Committee. Polymorphism Asp298Glu of the gene NOS3 (polymorphism Glu298Asp of endothelial nitric oxide synthase gene) was identified through the method of analyzing of the length of the fragments amplified and polymorph fragments of restriction.

Results

At children with overweight/obesity both hypertensives and normotensives was registered a greater frequency of the genotype AG of NOS3, but the proportion of the homozygous children according to $\mathrm{G}$ allele was greater at hypertensives $(25,81 \%)$, than at normotensives $(10,34 \%)$ and minimal in control group $(2,00 \%)$, where dominated carrying of the genotype AA $(46,00 \%)$. Homo/heterozygous holders of $\mathrm{G}$ allele showed higher chances of realization of arterial hypertension (OR 9,71; 95\% CI: 3,05-32,98; p0,001), they had advanced degree of obesity, mainly abdominal, dyslipidemia and higher serum values of insulin than homozygous holders according to A allele.

\section{Conclusion}

Our results suggest that at children with overweight/obesity carrying homo/heterozygous of G allele of NOS3 the risk of realization of arterial hypertension and metabolic disorders is higher. Appreciation of the polymorphism of this gene at children with overweight will initiate taking measures in the early stages.

\section{5}

\section{Genetic \& Metabolic}

A Complex VACTERL related malformation: aganglionosis, ilealcolonic atresia, recto-vestibular fistula and duplex kidney in a newborn Georgina Mata ${ }^{1}$, Gerard Roy ${ }^{2}$, David Croaker ${ }^{3}$

${ }^{T}$ Paediatrics and Child Health, Sydney Children's Hospital, Australia

${ }^{2}$ Paediatric Surgery, John Hunter Hospital, Australia

${ }^{3}$ Paediatrics and Child Health, The Canberra Hospital, Australia

\section{Aim of the study}

To describe an unusual and challenging anorectal malformation. 


\section{Methods}

Case report by review of records.

Main results

We report of a case of a female infant, born at 40 weeks gestation with a birth weight of $4.065 \mathrm{~kg}$, and ileal atresia with absent ileum and colon down to the sigmoid, leaving $150 \mathrm{~cm}$ of small bowel. There was aganglionosis of the remaining microcolon, imperforate anus with recto-vestibular fistula and right duplex kidney. The kidney demonstrated upper pole hydronephrosis and an unusual blood supply prevented the planned heminephrectomy. Antenatal ultrasounds elsewhere at 11 and 20 weeks of gestation were reported to be normal. The child was not obviously otherwise dysmorphic. Management over the first ten years of life involved more than 30 anaesthetics, 49 hospital admissions, and 135 hospital contacts. Perhaps related to this, the patient developed selective mutism for a period.

\section{Conclusion}

Hirschsprung's disease has been noted to be associated with a range of syndromic conditions. We propose that the underlying defect in this case is VACTERL related, with the distal aganglionosis secondary to the barrier to ganglion cell migration represented by the atresia which is sometimes associated with VACTERL. We note that the combination of pathologies in our patient has rarely if ever been previously described.

Management has been extremely challenging, but a degree of continence and stability has been achieved and with the patient now 12 years old, the family do not seem to regret their decision to ask for complete reconstruction.

604

\section{Genetic \& Metabolic}

Diagnosis of Rare Sphingolipid Storage Disease Mimicking Recurrent Septic Arthritis

Eftychia Ioanna Dimitrakopoulou ${ }^{I}$, Margarita Pesmatzoglou ${ }^{l}$, Aggeliki Korbaki ${ }^{I}$, Eleni Angelopoulou', Irene Orfanou ${ }^{I}$, Evanthia Botsa ${ }^{I}$

${ }^{I}$ First Pediatric Department, "Aghia Sophia Children's Hospital, University of Athens, Greece

\section{Background}

Gaucher's disease (GD) is an autosomal recessive disorder with genetic and phenotypic variability. It is the most prevalent sphingolipid storage disease, characterized by accumulation of glucocerebrosidase in macrophage-monocyte cells known as 'Gaucher cells', due to enzyme deficiency encoded by GBA-gene on chromosome-1.

\section{Objective}

Presentation of a case of GD with rare clinical presentation and rare mutation on the genetic analysis of GBA-gene.

Methods

A 12 year-old Caucasian boy presented with recurrent episodes of left hip pain and gradually increasing limp. He was developmentally normal, born to non-consanguineous parents. From his past medical history he had undergone surgical debridement and drainage of a soft tissue infection of the left femoral area.

There was no clear history of trauma and no significant family history of any disorder.

On physical examination at presentation restricted range-of-movement of the left hip was noted as well as painful weight-bearing and antalgic gait; there was mild splenomegaly, no hepatomegaly, no lymphadenopathy and no signs of neurological abnormalities. The rest of systemic examination was essentially normal. Laboratory investigations including fbc, liver and kidney function test and urine analysis were unremarkable. Blood and joint fluid cultures were negative. X-ray of the joint was normal. The joint-ultrasound revealed joint effusion. The patient was initially treated as septic arthritis. He showed deterioration of his clinical condition on ambulation and from the CT/MRI femoral head necrosis was noted needing surgical intervention. A bone marrow biopsy was performed which revealed Gaucher cells. Elevated plasma chitotriosidase confirmed the diagnosis of GD. Genetic analysis of GBA-gene showed the patient was heterozygote for two different known mutations NS370S/ IVS6-2A-G which is consistent with Type 1 GD.

\section{Conclusion}

GD should be considered in the differential diagnosis of patients with unexplained bone pathology. The early recognition of GD leads to early treatment with enzyme-replacement which can decrease morbidity.

\section{9}

\section{Genetic \& Metabolic}

Case report of an patient with recurrent cerebral edema cause by medium-chain acyl-coenzyme a dehydrogenase deficiency

Nicoleta Aurelia Popescu ${ }^{1}$, Maria Gutu ${ }^{I}$, Nicoleta Aurelia Popescu ${ }^{l}$, Adrian Stanciu ${ }^{l}$, Adrian Stanciu ${ }^{I}$

${ }^{1}$ Pediatric, Clinical Emergency Hospital for Children Maria Sklodowska Curie, Romania

\section{Background}

Recurrent cerebral edema is a rare pathology in adults and children. Metabolic diseases associated with genetic defects are one of the causes that have been found in recurrent cerebral edema.

\section{Case study}

1-year-old patient, male, known with severe global developmental delay, central hypotonic syndrome and genetic syndrome (1p36 deletion) is admitted for tonic-clonic seizures associated with fever $(\mathrm{T}=38.9$ degrees Celsius). After preclinic and imagistic evaluation (laboratory tests and cerebral CT,EEG), the diagnosis is massive intra-infectious acute cerebral edema. Under treatment with antibiotic, antiviral, brain depletive and anticonvulsant, the evolution was initially favorable. When the treatment was discontinued, the patient presented recurrent cerebral edema.

Results

After deep investigations, a metabolic cause is found - MCAD (Mediumchain acyl-coenzyme A dehydrogenase deficiency) with carnitine deficiency in a child with genetic syndrome.

\section{Discussion}

Metabolic causes remain in discussion for recurrent cerebral edema when the etiology of tumor, malformations, vascular diseases, infections, especially tuberculosis, have been excluded.

Keywords: recurrent cerebral edema, MCAD, carnitine deficiency, 1p36 deletion.

\section{8}

\section{Genetic \& Metabolic}

Girl With Ectopia Lentis, and Possibly Marfan Syndrome, caused by a FBN1 Gene Mutation

Ulrich Zechner ${ }^{1}$, Oliver Bartsch ${ }^{1}$, Sorin Ioan Iurian ${ }^{2}$

${ }^{1}$ Institute of Human Genetics, Johannes Gutenberg University, Mainz, Germany;

${ }^{2}$ Pediatric Hospital, Faculty of Medicine, "Lucian Blaga” University of Sibiu, Romania

\section{Background}

Marfan syndrome (MFS) and Ectopia Lentis, which is part of the MFS spectrum of fibrillinopathies, are allelic autosomal-dominant connective tissue disorders caused by mutations in the Fibrillin 1 gene (FBN1). FBN1 enables normal development of ocular, cardiac and musculoskeletal structures. Almost inevitable, most severe complications of MFS include aortic root dilatation, aortic dissection and sudden cardiovascular death. To alleviate the clinical course, early diagnosis and monitoring are essential. 


\section{Objective}

We performed molecular diagnostics in a 4-year-old girl with bilateral lens dislocation but no other signs of MFS.

\section{Methods}

The girl was the only affected in her family. Clinical examination revealed no skeletal, cutaneous, cardiovascular, or pulmonary abnormalities. According to the revised Ghent criteria (2010), patients without positive family history should demonstrate four different clinical abnormalities to establish a diagnosis of MFS (revised criteria give more weight to aortic aneurysm and lens dislocation than original criteria). This girl did not meet the Ghent criteria, but nevertheless we strongly suspected mild or incomplete MFS and performed molecular genetic analysis.

\section{Results}

Using next generation sequencing we identified a heterozygous base exchange c.4589GC in FBN1. The variant was not reported previously. It was absent in the parents and thus likely originated de novo in the patient. We propose that it represents a novel pathological (disease-causing) mutation. Furthermore the girl demonstrated a heterozygous c.4645CT variant (predicting p.Arg1549Cys) in MYLK (the gene for aortic thoracic aneurysm, type 7) inherited from the mother. This $M Y L K$ variant was also not described previously and was predicted to likely represent another pathological finding.

\section{Conclusions}

(1) Bilateral ectopia lentis represents an important diagnostic clue to MFS spectrum disorders. (2) Early diagnosis is crucial to delay complications. (3) Additionally we identified in the girl and her mother a MYLK gene variant that could possibly represent another genetic predisposition for aortic aneurysm.

197

\section{Genetic \& Metabolic}

Hidden neurodiversity in paediatric practice: Klinefelter Syndrome Cillian Lineen ${ }^{1}$, Uzair Khan ${ }^{1}$, Mahmoud Hassan ${ }^{1}$, Anne Marie Murphy ${ }^{1}$ ${ }^{1}$ Department of Paediatrics, Univeristy Hospital Limerick, Ireland

\section{Introduction}

Klinefelter syndrome has a reported incidence of 1 in 500 live male births. It is commonly diagnosed in adulthood but the majority of cases go undiagnosed. Early diagnosis and intervention can be of medical, psychological and fertility benefit.

Aim

Our aim is to present four unexpected cases of Klinefelter Syndrome who presented to general paediatrics in an effort to raise awareness of this under recognised phenomenon.

\section{Methods}

Clinical details and natural history of all four patients were identified from hospital records. A literature search was conducted on the optimal management of Klinefelter syndrome.

\section{Results}

Case one was diagnosed at the age of 18 months with global developmental delay, plagiocephaly and absent testes. His karyotype demonstrated 49 XXXY.

Case two (47 XXY, with deletions on chromosomes 14 and 19) was diagnosed at the age of 10 years. His karyotype was tested because he had an extensive family history of chromosomal deletions and his family wished to know if he had the same. He had a history of motor delay (including hypotonia since birth) and cryptorchidism.

Case three $(47 \mathrm{XXY})$ was diagnosed at the age of 5 years with a history of social, psychological and speech delay. He had a history of emotional problems and tantrums.

Case four $(47 \mathrm{XXY})$ was diagnosed at the age of 11 months, after presenting with macrocephaly and plagiocephaly, with a normal neurological exam and normal brain MRI.

\section{Conclusion}

Klinefelter syndrome can present variably and subtly. Increased clinical suspicion can detect cases more frequently and earlier, providing optimal management options.

\section{4}

\section{Genetic \& Metabolic}

Successful Resection of Rapidly Developing Right Atrial Myxoma in a Patient with Familial Carney Complex

Rachel Power $^{\text {I, Jonathon McGuinness }}{ }^{1}$, Colin McMahon ${ }^{1}$

'Paediatric Cardiology, Our Lady's Children's Hospital Crumlin, Ireland

\section{Background}

Carney syndrome is a rare autosomal dominant inherited neoplastic condition characterised by atrial myxomas, buccal and cutaneous lentigines and non-cardiac neoplasia. The condition arises secondary to a mutation in the PRKAR1A gene. Successful surgical resection has been reported in several cases.

\section{Objective}

This case highlights successful surgical resection of a rapidly growing tumour including the atrial septum, where the tumour was anchored.

\section{Methods}

We report a case of a rapidly growing atrial myxoma in a patient with Carney Complex and review contemporary literature.

\section{Results}

A 14 year old young man with Carney syndrome initially presented to the clinic for screening for atrial myxoma.. Three generations of family members were affected including the patients' mother and grandmother who previously underwent surgical resection of a left atrial myoma.

Annual screening failed to detect any intracardiac lesion until the patient presented at 17 years of age with a massive right atrial myxoma(Image 1). The tumour measured $30 \times 50 \mathrm{~mm}$. Surgical resection was undertaken via a median sternotomy(Image 2). Despite the rapid growth of the tumour the patient recovered well and is now asymptomatic.

\section{Conclusion}

In conclusion patients with Carney complex have the potential for rapid growth of an atrial myxoma, coexistent tumours, and a high risk of recurrence. Close serial surveillance of patients with this condition is warranted.

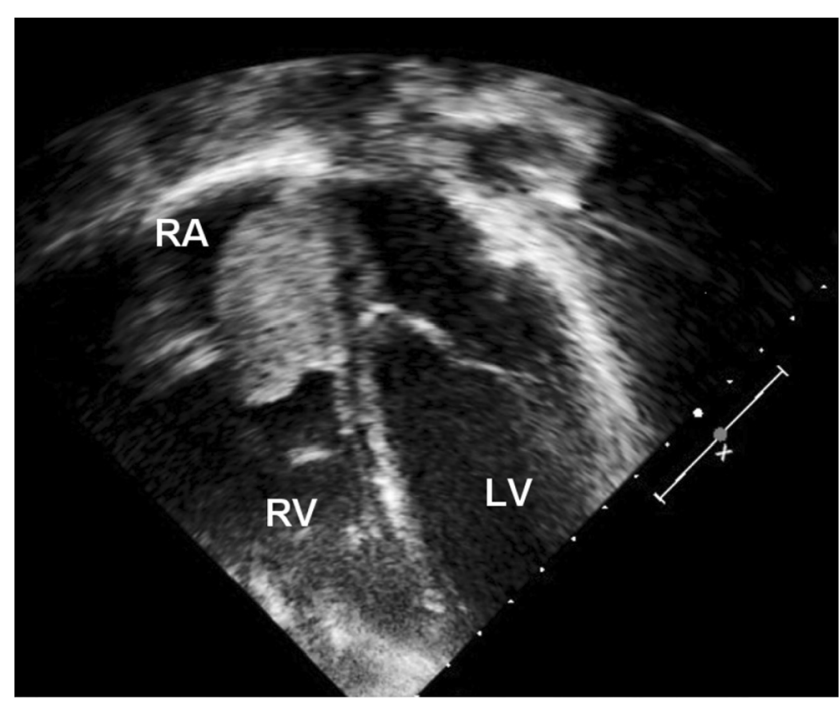




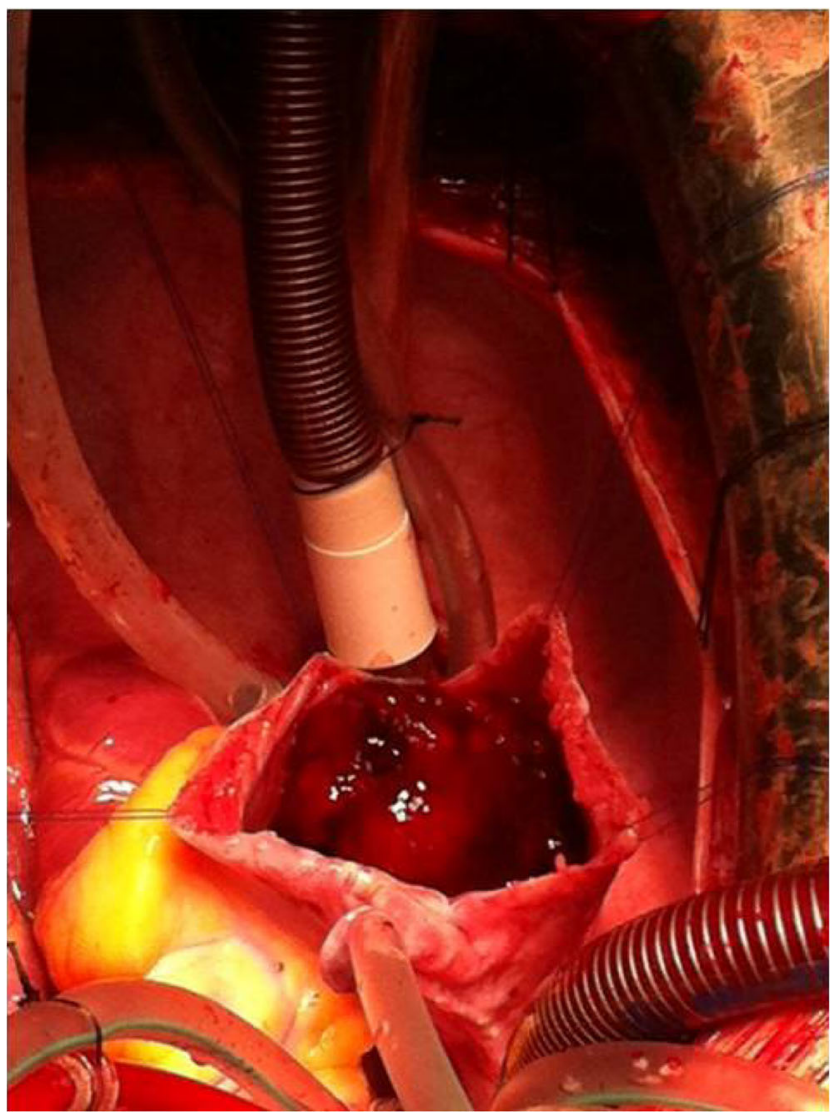

366

Genetic \& Metabolic

Novel Skeletal Features in Siblings with Schimke Immuno-Osseous Dysplasia

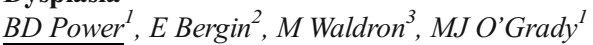

${ }^{T}$ Dept. of Paediatrics, Regional Hospital, Mullingar, Co. Westmeath, Ireland ${ }^{2}$ Dept. of Nephrology, Midland Regional Hospital, Tullamore, Co. Offaly, Ireland ${ }^{3}$ Dept. of Paediatric Nephrology, Our Lady's Children's Hospital, Crumlin, Dublin, Ireland

\section{Background}

Schimke Immuno-Osseous dysplasia (SIOD) due to SMARCAL1 mutation is an autosomal recessive disorder with a prevalence of one in 1 to 3 million. It is characterised by a diagnostic triad of spondyloepiphyseal dysplasia, T-cell immunodeficiency and progressive renal disease.

\section{Objective}

To report novel skeletal features in a sibship with genetically confirmed SIOD. Methods

Case notes were summarised and a review of previously published cases of SIOD was undertaken.

\section{Results}

The female proband presented with failure to thrive in infancy and echocardiogram revealed a perimembranous ventricular septal defect (VSD). Marked proteinuria was identified at age 5 and renal biopsy subsequently confirmed Focal-segmental glomerulosclerosis (FSGS). Physical examination was notable for short stature, dysmorphic features, Klippel-Feil syndrome and a Sprengel shoulder. She progressed to end-stage renal failure in adolescence. Her clinical course also included an ischaemic stroke from cerebral vasculopathy, hypothyroidism and T-cell immunodeficiency. Heterozygous c.2114CT and c. $2070+2$ _3insT mutations in the SMARCAL1 gene were identified. The probands younger brother had incidental detection of mild proteinuria in early adolescence. His physical and skeletal features are similar to those of the proband. $\mathrm{He}$ has subclinical hypothyroidism and laboratory evidence of T-cell immunodeficiency. His renal function is currently normal aged 19 years. Genetic analysis confirmed identical mutations to his sister.

\section{Conclusion}

To date, no published case has outlined cardiac septal defects as a clinical feature of SIOD. Klippel-Feil syndrome and Sprengel shoulders in patients with SIOD have not been previously described in the literature, but cardiac abnormalities can be a feature of Klippel-Feil syndrome. In addition, this case series describes both a case of early-onset SIOD with multiple, lifethreatening co-morbidities and a case of juvenile-onset SIOD with a significantly milder phenotype despite an identical genotype.

\section{4}

\section{Genetic \& Metabolic}

Hunter syndrome- 11 years of enzyme replecament therapy in Estonian patient Riina MD, PhD Zordania ${ }^{1}$, Valentin MD Sander ${ }^{2}$, Kairit MD, PhD Joost ${ }^{3}$ ${ }^{T}$ Tallinn, Tartu University Hospital UL Centre of Clinical Genetics, Estonia ${ }^{2}$ Neurology, Tallinn Children' Hospital, Estonia

${ }^{3}$ Molecular Genetics, Asper Biotech Ltd, Estonia

Hunter syndrome (mucopolysaccharidosis II, MPS II) is a rare, X-linked metabolic disease caused by a deficiency of the lysosomal enzyme, iduronate-2sulfatase (I2S).The clinical spectrum of Hunter syndrome spans a wide range, from an attenuated to a severe phenotype.Patients usually appear normal at birth, but symptoms typically begin to appear between 2 and 4 years of age. All affected patients experience progressive course of disease including hepatosplenomegaly, skeletal and joint involvement resulting in reduced joint range of motion and contractures, and heart and airway disease. Recombinant human I2S (idursulfase) was approved for the treatment of Hunter syndrome in 2006. Idursulfase is administered intravenously on a weekly $(0.5 \mathrm{mg} / \mathrm{kg})$. Improvements included reduction in urinary GAG excretion, reduction in liver and spleen volume, and improvements in respiratory function.

Aim- to present clinical and laboratory data of the Estonian MPSII patient, who has been on enzyme replacement treatment (ERT) during 11 years.

Clinical data: child presented at the age of age 3.5 years with coarse face, hepatomegaly $(+3 \mathrm{~cm}$ from costal margin). Gradually developed joint stiffness, restricted movements and hearing loss. ERT was started at the age of 10 years with the weekly infusion $0.5 \mathrm{mg} / \mathrm{kg}$.

Results: Following 11 years of ERT at the age of 21 years patient has short stature ( $-2 \mathrm{SD})$, minimal hepatomegaly and no cardiomegaly. Urinary GAG excretion is within reference range and disease has shown no significant progression. Detailed data are presented.

Conclusion: Idursulfase has modified significantly the the natural course of MPS II in patient. As effective disease modifing treatment is available for MPS II early identification of patients is crucial to improve final outcome of patients.

500

Hemato - Oncology

Bone Demineralization, Iron Overload and Time to Engraftment following Allogeneic Stem Cell Transplantation in Pediatric Patients Giulia Bravar ${ }^{1}$, Massimo Gregori ${ }^{2}$, Davide Zanon ${ }^{2}$, Roberto Simeone ${ }^{2}$, Natalia Maximova ${ }^{2}$

${ }^{1}$ Institute of Maternal and Child Health, University of Trieste, Italy ${ }^{2}$ Institute of Maternal and Child Health, IRCCS-Burlo Garofolo, Italy

\section{Background}

Iron overload and osteoporosis are relatively common but often neglected complications of chemotherapy and transplantation ${ }^{3,4,5}$. 
Under these pathological conditions bone marrow-derived mesenchymal stem cells (BM-MSCs) exhibited deficiencies in proliferation and imbalances in osteogenic/adipogenic differentiation, along with reduced expression of common hematopoietic cytokines chemokines and adhesion proteins ${ }^{2}$. This can induce impaired production of hematopoietic progenitors and at the end compromise engraftment.

\section{Methods}

The medical records of 41 pediatric patients who underwent bone mineral density (BMD) measurement and bone iron concentration (BIC) evaluation prior to allogeneic HSCT from January 2013 to December 2016 were retrospectively reviewed. Magnetic resonance imaging was used to estimate BIC and dual-energy X-ray absorptiometry was used to measure BMD. Patients were divided into three groups, 17 with non-elevated BIC $(100 \mu \mathrm{mol} / \mathrm{g})$ and normal BMD (Group 2) and 10 patients with elevated BIC $(100 \mu \mathrm{mol} / \mathrm{g})$ and pathological BMD at baseline. The endpoint of our study was to compare times to engraftment in the three groups.

Results

Pre-transplant BIC and BMD were significantly associated with neutrophil and platelet engraftment, with mean times to engraftment of both platelets $(22.1 \pm 9.5$ days vs. $42.0 \pm 35.3$ and $44.6 \pm 31.5$ in three groups respectively; p0.05) and neutrophils $(17.8 \pm 4.6$ days vs. $22.8 \pm 8.8$ and $23.1 \pm 10.4$ days in three groups respectively, p0.05) being significantly longer in Group 2 and 3 than in Group 1. Time to platelet engraftment showed statistically significant correlations with pre-transplant BIC $(\mathrm{r}=0.7305 ; \mathrm{p} 0.001)$. Pre-transplant BIC also showed close correlations with mean times to neutrophil engraftment (p0.001).

\section{Conclusion}

Our findings show a correlation between pre-transplant BIC, BMD and time to engraftment. Further studies are needed to determine whether normalization of BMD and BIC could reduce time to engraftment and therefore transplant-related morbidity and mortality.

\section{6}

\section{Hemato - Oncology}

An Audit of a National Paediatric Cardiology Warfarin Clinic Catherine Breen ${ }^{I}$, Siobhan McCormack ${ }^{1}$, Professor Colin McMahon ${ }^{1}$ ${ }^{T}$ Cardiology, Our Lady's Children's Hospital Crumlin, Ireland

\section{Background}

The National Cardiology Heart Centre manages the most complex cardiology conditions in Ireland leading to a large cohort of patients requiring anticoagulation. The warfarin clinic is a telephone clinic managed by the Cardiology medical team.

Aim

To audit the Warfarin Clinic using the NPSA (National Patient Safety Authority) guidelines established by the NHS for warfarin dosing.

Method

The computerized data collection of all warfarin patients was reviewed over a three month period. This revealed 54 patients on warfarin with a total of 495 INRs. Data recorded from the telephone clinic was audited under the heading of key safety indicators as outlined by the NPSA. The method of monitoring the INR was also audited by telephone when contacting parents with doses. The results were compared to the NPSA safety standards for warfarin dosing. A standard of $90 \%$ was set for each standard

Results

Fifty-four patients were included in the audit. One hundred percent of patients had a documented target INR, clinical diagnosis and most recent doses recorded. No patient had a documented stop date. Sixty-nine percent of INR's were in target range. $7 \%$ $(N=4)$ had an INR of greater than 5 and no patients had an INR greater than 8 . No patients had an adverse outcome. There was no process for identifying patients lost to follow up. Thirty-three percent of 30 patients audited are using home 'coagucheck' kits with the remaining audited patients using their local hospital phlebotomy services.

\section{Conclusion}

This audit shows that there is a need to implement a computerized system of following up patients. An online calendar has been set up which documents what date patients are booked in for INR review. All new patients will now have a stop date documented. There will be a re-audit in three months' time with the aim of improving compliance to the safety standards.

\section{9}

\section{Hemato - Oncology}

Effect of Bovine Colostrum on the Absolute Neutrophil Count of Pediatric Patients with Acute Lymphocytic Leukemia undergoing Chemotherapy - A Double Blind Randomized Placebo Controlled Study Edith Cyrill Caysido ${ }^{l}$, Edith Cyrill Caysido ${ }^{1}$

${ }^{T}$ Department of Pediatrics, Baguio General Hospital and Medical Center, Philippines

\section{BACKGROUND}

Changes in the blood counts mainly leukopenia and neutropenia in patients with Acute Lymphoblastic Leukemia (ALL) are common adverse events following chemotherapy. These commonly delays further administration of chemotherapeutic agents thereby potentially affecting therapeutic outcomes. Bovine colostrum has shown some promises in different fields of medicine and one claim is its use in prevention of neutropenia. However, there are no studies to support such claim

\section{OBJECTIVE}

The general objective of this study is to determine the efficacy of a 1 week supplementation of bovine colostrum in preventing neutropenia among patients with ALL undergoing standard chemotherapy.

\section{METHODOLOGY}

This is a randomized, double blind, placebo controlled study involving the use of bovine colostrum for 1 week against placebo in preventing neutropenia among patients aged 6 months to 18 years old diagnosed with ALL, and undergoing chemotherapy using the standard protocol. Participants were randomly assigned to receive either the bovine colostrum of the placebo by fishbowl technique which were given orally $2 \mathrm{x}$ a day for 7 days starting simultaneously with the chemotherapy before the outcome measures were evaluated.

\section{RESULTS}

A total of 21 subjects were enrolled, 10 of them received the placebo while 11 received the bovine colostrum. Majority in both treatment group belonged to the age group of 1-9 yrs old and most are male. There was significant increase in the Absolute Neutrophil Count (ANC) of patients given bovine colostrum as compared to the placebo group. There was also noted significant increase in the $\mathrm{WBC}$ and platelet counts among those who were given Bovine Colostrum. No incidence of infection or untoward effects on both treatment groups.

\section{CONCLUSION}

This study shows that Bovine Colostrum is effective and safe in preventing leukopenia, neutropenia and thrombocytopenia among ALL patients undergoing chemotherapy thereby preventing delays in chemotherapy.

Keywords: neutropenia, ALL, ANC, bovine colostrum, chemotherapy 
505

\section{Hemato - Oncology}

Unusual Presentation of Paediatric Burkitt's Lymphoma: Acute Pancreatitis and the Sequelae - a Case Report

Deevena Chinthala ${ }^{1}$, John Tolliday ${ }^{1}$, Alastair Baker ${ }^{2}$, Ramya Ramanujachar

${ }^{1}$ Paediatric Haem-Oncology, University Hospital Southampton, UK

${ }^{2}$ Paediatric Hepatology, Kings College Hospital, UK

\section{Introduction}

Non-Hodgkin Lymphoma (NHL) is the third most frequent cancer of childhood. We report an unusual presentation of Paediatric Non Hodgkin Lymphoma as acute pancreatitis and the sequelae.

Case report

An eight year old boy was referred with acute pancreatitis. He had a history of evolving multiple subcutaneous scalp nodules, fever, weight loss, abdominal pain, jaundice and renal impairment for a month. Cross sectional imaging demonstrated bilateral pleural effusions, ascites, hepatosplenomegaly, enlarged pancreas causing obstruction of the common bile duct, thickened stomach wall and enlarged kidneys.

Stomach wall and scalp nodule biopsies confirmed $t(8 ; 14)$ B-NHL and the disease was staged as high-risk Stage IV.

Treatment with modified chemotherapy for hepatic and renal dysfunction based on CCLG(Children's Cancer and Leukemia Group) recommendations was commenced. Further response assessment showed necrosis in the head of the pancreas and pseudocyst formation in the tail of the pancreas. He subsequently required Insulin for endocrine pancreatic insufficiency.

He then required treatment for with invasive pulmonary aspergillosis and systemic bacterial infections which worsened the hepatic dysfunction. He developed progressive obstructive jaundice with peak bilirubin of $108 \mathrm{umol} / \mathrm{L}$ after five cycles of chemotherapy. A Magnetic Resonance Cholangiopancreatogram demonstrated distal common bile duct obstruction with pancreatic necrosis and pseudocyst. He has now completed treatment and has necrotic nonfunctional chronic pancreatitis. The liver function has improved after placement of a stent in the common bile duct.

Imaging:

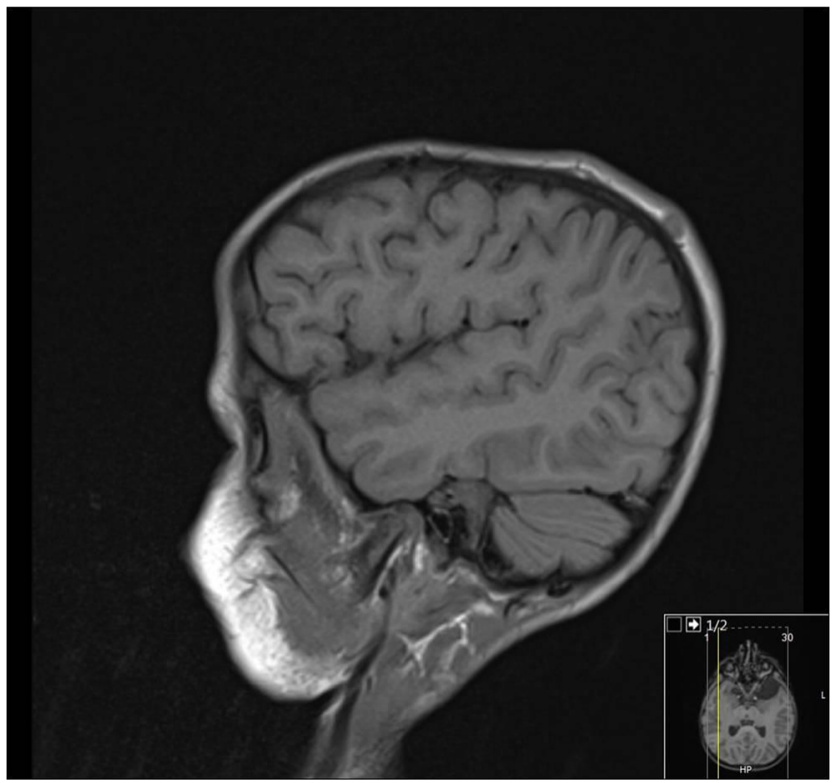

MRI head- scalp lesions

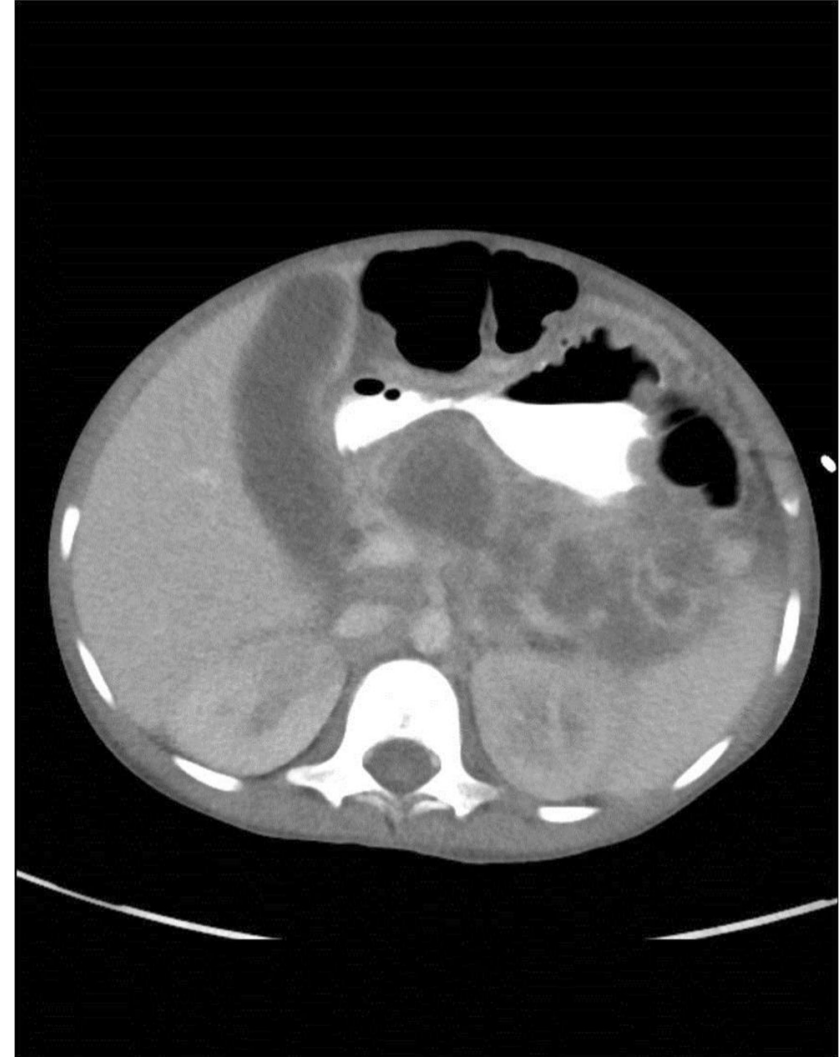

CT abdomen- enlarged pancreas

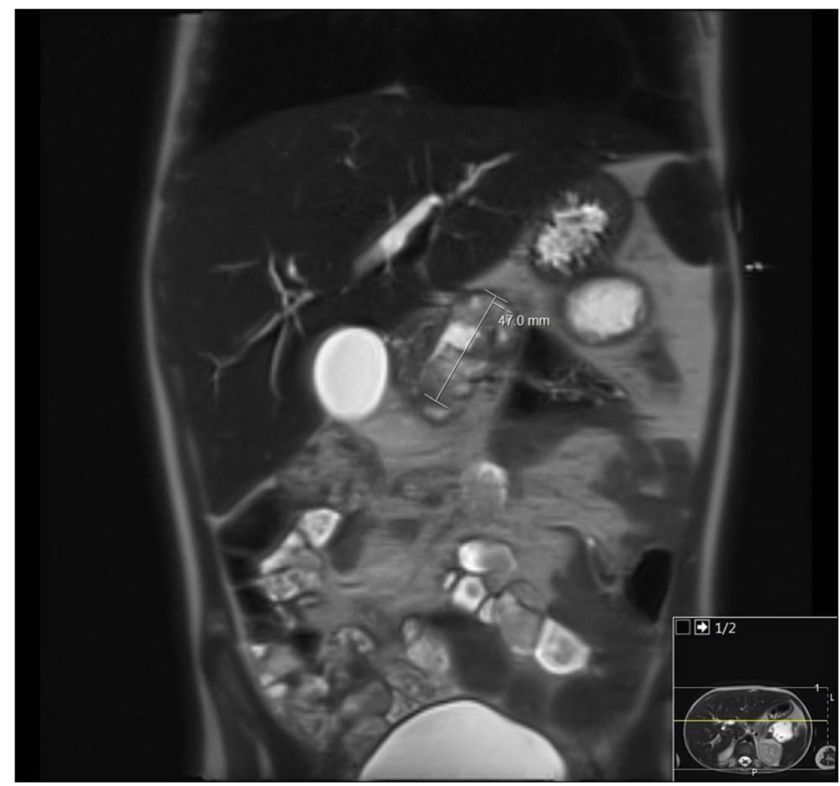

MRCP- pancreatic pseudocyst 


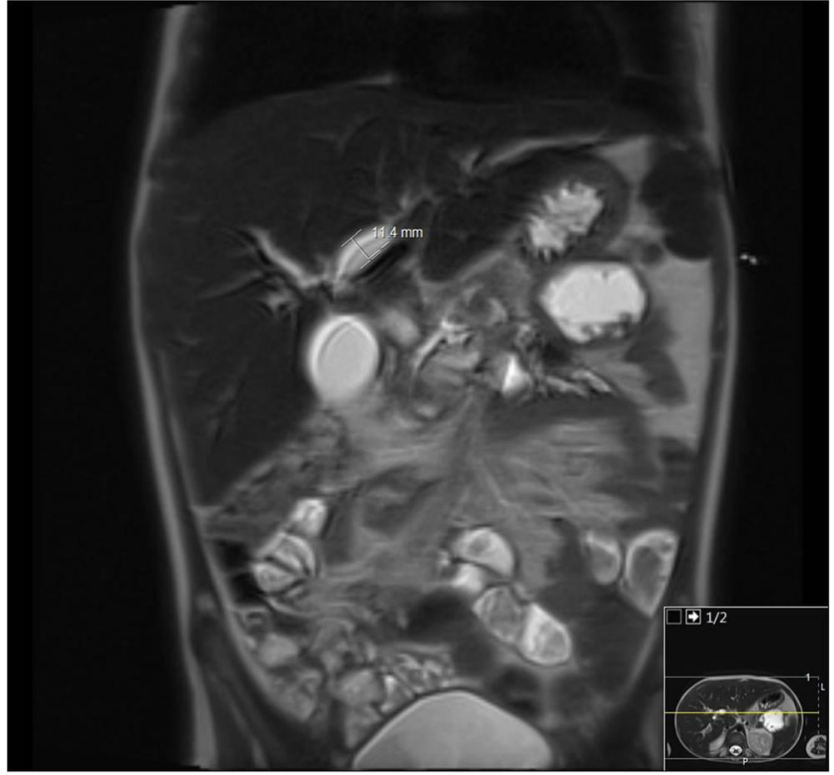

MRCP- dilated CBD

\section{Discussion}

Acute pancreatitis in children is secondary to medications, trauma, anatomical abnormalities or autoimmune phenomena. Only less than $2 \%$ of Paediatric NHL patients have pancreatic involvement.Our patient represents an unusual presentation of pancreatitis and sequelae of Burkitt's Lymphoma, the first case report in literature.

Reference:

Koca T. et al., Burkitt lymphoma with unusual presentation: Acute pancreatitis. Pediatr Int. 2015 Aug;57(4):775-7,Epub 2015 May 29.

\section{3}

\section{Hemato - Oncology}

Invasive Zygomycosis during Treatment for Acute Lymphoblastic Leukaemia - Management of Two Life Threatening Diseases

A. Trobisch $^{l}$, V. Strenger ${ }^{l}$, B. Kohlmaier ${ }^{I}$, M. Egger ${ }^{l}$, S. Kurath-Koller ${ }^{I}$, $\overline{\text { H. Lackner }}^{1}$, A. Karastaneval, E. Sorantin ${ }^{2}$, C. Urban ${ }^{1}$

${ }^{1}$ Department of Paediatric and Adolescent Medicine, Division of Paediatric Haemato-Oncology, Medical University Graz, Austria ${ }^{2}$ Department of Radiology, Division of Paediatric Radiology, Medical University Graz, Austria

\section{Background}

Zygomycetes cause acute angioinvasive infections and have mortality rates $60 \%$, depending on underlying condition.

\section{Case Presentation Summary}

A 5-year-old patient with acute lymphoblastic leukaemia (ALL) under treatment according to AIEOP-BFM-ALL 2009 protocol developed suspected pulmonary mycosis. After stop of ALL treatment and initiation of antimycotic treatment with liposomal Amphothericin B (AmBisome) and Voriconazole the patient was transferred to our clinic. After admission, thoracoscopic biopsy was performed. Specimens showed fungal hyphae, fungal PCR (ITS/5,8SPCR) and sequencing revealed Actinomucor elegans representing proven invasive zygomycosis according to EORTC criteria. Therefore, Voriconazole was switched to Posaconazole $(15 \mathrm{mg} / \mathrm{kg} / \mathrm{d}$, target drug levels of 0.5-5 mg/l) and dosage of AmBisome was increased to $10 \mathrm{mg} / \mathrm{kg}$. After 4 weeks without ALL treatment, an interval therapy with Methotrexate and 6Mercaptopurine was administered for another month. Under continued antimycotic treatment with AmBisome, we reinitiated therapy according to the AIEOP-BFM-ALL 2009 protocol, completed block Ilb and started maintenance therapy. Pulmonary zygomycosis was monitored every 2-6 months by thoracic MRI (avoiding radiation of $\mathrm{CT}$ ) with additional intermittent thoracic $\mathrm{CT}$ and PET-CT scans. In long term, AmBisome dosages were reduced, and intervals were extended to every 3rd day due to practicability and to reduce possible side effects during myelodepressive chemotherapy. ALLtreatment was terminated 22 months after diagnosis ( 2 months earlier than required) in order to improve immune system. Since flow-cytometry showed continuously diminished B- and T-cell fractions, AmBisome was given for another 7 months post ALL-treatment. Recent thoracic CT indicates further improvement with primarily signs of tissue scarring.

\section{Learning Points/Discussion}

Despite invasive pulmonary zygomycosis, ALL treatment has to be continued under intensive antimycotic therapy in order to control these two potentially lethal diseases. Thoracic imaging and immune monitoring help in guiding treatment decisions.

580

\section{Hemato - Oncology}

Using the International Society of Paediatric Oncology minimum standards of paediatric oncology nursing care recommendations for low- and middle-income countries to identify priorities in Myanmar Jay Halbert ${ }^{1}$, Michael Malley, ${ }^{1}$, Marie Monaghan ${ }^{1}$

${ }^{T}$ Global Child Health, Royal College of Paediatrics and Child Health, UK

\section{Aims}

In 2016 the International Society of Paediatric Oncology (SIOP) published recommendations for the minimum standards of paediatric oncology nursing care in low- and middle-income countries. Here we review how these guidelines may be used to identify local successes and priorities at a paediatric oncology department in Myanmar.

\section{Methods}

In August 2016 a visiting international team together with local paediatric oncologists and nurses performed a review of a local paediatric oncology department in Myanmar. The new SIOP minimum standards of paediatric oncology nursing care recommendations for low- and middle-income countries were used to guide the review.

\section{Results}

The table below summarises the six core minimum standards and how they relate to local successes and challenges at Mandalay Children's Hospital.

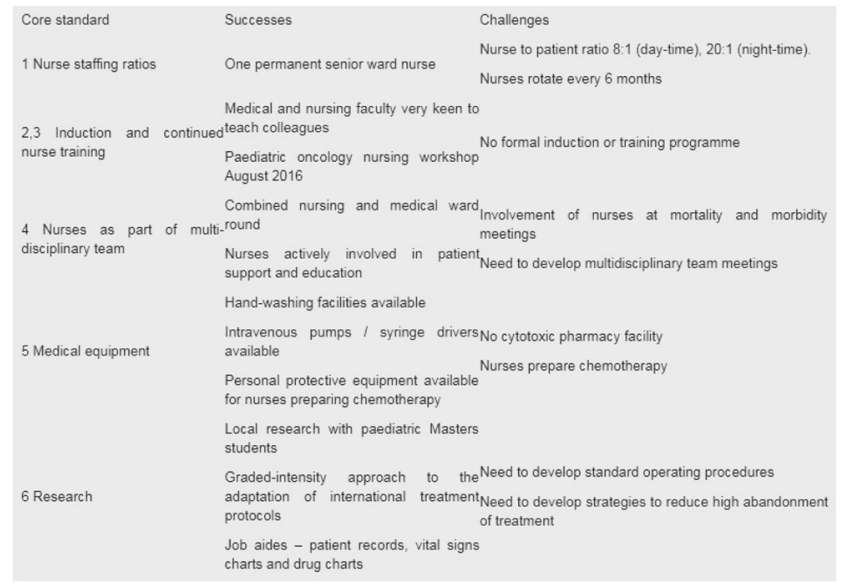

\section{Conclusion}

The 2016 SIOP minimum standards of paediatric oncology nursing care recommendations for low- and middle-income countries is a useful tool to identify local needs and guide service development. 
493

\section{Hemato - Oncology}

Prevalence and significance of BRAF alterations in a pediatric population with low-grade gliomas

Ilaria Liguoro $^{1}$, Chiara Pilotto ${ }^{1,2}$, Raffaello Tosolini ${ }^{1,2}$, Eva Passone $^{l}$, Paola Cogo

${ }^{1}$ Pediatric Clinic, Department of Maternal and Child Health, ASUIUD S. Maria della Misericordia, University of Udine, Italy

${ }^{2}$ Department of Medical and Biological Science, University of Udine, Italy

\section{Background}

Low-grade gliomas (LGG) are the most common brain tumours in children, represented by heterogeneous pathological entities. BRAF gene alterations have been recently identified as responsible of constitutive activation of the mitogen-activated protein kinase pathway (MAPK) and hence involved in the development of LGG in children. There is evidence that the BRAF V600E mutation is more common in supratentorial LGG while the KIAA1549:BRAF fusion in posterior fossa pilocytic astrocytoma (PA).

Objective

This case series describes the prevalence of BRAF alterations in LGG patients, trying to relate them to outcome.

Methods

Children aged $0-14$ years, with a diagnosis of LGG, referred to a single neuro-oncologic centre, were retrospectively reviewed to analyze clinical and histopathological features related to BRAF alterations.

\section{Results}

A total of 35 patients were included (16 males, median age $85.5 \pm 81.3$ months). BRAF mutations were searched on $7 / 35$ children $(20 \%)$ resulting positive in $5 / 7$ (71\%). Two of them (40\%) showed the KIAA1549:BRAF fusion. They were both pilocytic astrocytomas located in posterior fossa: $1 / 2(50 \%)$ was totally resected, showing stable disease (SD) 1 year after surgey; the other was not surgically treatable and showed a progressive disease during chemotherapy. Three out of five $(60 \%)$ presented V600E mutation. All of them were supratentorial: 1 pilomyxoid astrocytoma, treated with two partial resections and subsequent chemotherapy, was in SD 5 years after diagnosis; 1 diffuse pleomorphic xanthoastrocytoma underwent three partial resections and showed SD after 5 years of vemurafenib; 1 glioneuronal tumor was in SD after complete resection and radiotherapy.

\section{Conclusion}

Our data support the evidence that specific mutations of the $B R A F$ pathway are related to site and histological subtype of brain tumors. This sample is too small to help supporting the hypothesis that these alterations have a prognostic impact. Extent of surgery and localization seem to be the most important prognostic factors.

\section{5}

\section{Hemato - Oncology}

Primary intramedullary spinal cord tumors in children: a singlecentre experience

Ilaria Liguoro $^{1}$, Raffaello Tosolini ${ }^{1,2}$, Chiara Pilotto ${ }^{1,2}$, Eva Passone $^{l}$, Agostino Nocerino ${ }^{I}$, Paola Cogo ${ }^{1}$

${ }^{1}$ Pediatric Clinic, Department of Maternal and Child Health, ASUIUD S.

Maria della Misericordia, University of Udine, Italy

${ }^{2}$ Department of Medical and Biological Science, University of Udine, Italy

\section{Background}

Primary spinal cord tumors are rare, accounting for $4 \%$ to $8 \%$ of all Central Nervous System (CNS) neoplasms in the pediatric population.
Most of them are low grade tumors and prognosis is related to the extent of surgical resection.

Objective

The aim of this study was to evaluate the clinical outcome of a small group of patients with intramedullary spinal tumors.

\section{Methods}

We retrospectively analyzed clinical and histopathological features, treatment modalities, survival rates of children aged $0-14$ years with a diagnosis of spinal tumor treated at our department during the last 15 years. Results

A total of 93 patients with a CNS tumor were included. Four of them (4.3\%) had a spinal localization: 2 males and 2 girls, $3 / 4(75 \%) 5$ years of age at diagnosis (mean age $4.4 \pm 3.6$ years). Three $(75 \%)$ had cervical localization, one $(25 \%)$ was localized in the lumbosacral tract. All cases underwent surgery: 2 biopsies (50\%), 1 partial resection $(25 \%)$ and 1 total resection $(25 \%)$. All of them were low-grade gliomas: $2(50 \%)$ pilocytic astrocytomas, 1 (25\%) glioneuronal tumor and 1 ependymoma. All of the children with subtotal resection (biopsy and partial resection) underwent subsequent therapies: 2 were treated with radiotherapy (RT) and chemotherapy (CT), 1 is under medical treatment (SIOP-LGG 2004 protocol). Regimens of CT included Carboplatin-Vincristin in pilocytic astrocytomas, and Cisplatin-Temozolomide in glioneuronal tumor. At current time, all these patients had stable disease.

\section{Conclusion}

Our data support the evidence that the majority of intramedullary tumors are histologically benign and radical resection results in long-term survival. When total surgical resection is not achievable, excellent results in survival rates could be obtained with a multimodal treatment combining $\mathrm{RT}$ and $\mathrm{CT}$.

\section{0}

\section{Infectious Diseases}

Evaluation of the New Molecular Alere ${ }^{\mathrm{TM}}$ RSV Test for Rapid Detection of Respiratory Syncytial Virus (RSV) in Routine Conditions

Omar Kamel ${ }^{1}$, Sarah Jourdain ${ }^{2}$, Phu-Quoc L $\hat{e}^{2}$, Isabelle Thomas ${ }^{3}$, Ingrid ${\text { Beukinga }{ }^{I}, \text { Laurent Blairon }}^{I}$

${ }^{1}$ Department of Laboratory Medicine, Iris Hospitals South, Belgium

${ }^{2}$ Department of Paediatrics, Iris Hospitals South, Belgium

${ }^{3}$ National Influenza Reference Centre, Scientific Institute of Public Health, Belgium

\section{Background}

RSV is an important respiratory pathogen in children, but also in elderly adults with underlying cardiopulmonary diseases, in young adults, and in the immunocompromised. The epidemic period of RSV infection in Belgium stretches from early October to late February, with a peak in December and January. In our institution, the diagnosis of RSV is made with the immunochromatographic BinaxNOW® RSV assay during epidemic period and with the SimulFluor ${ }^{\circledR}$ direct immunofluorescence during the rest of the year. However, these methods lack sensitivity (estimated at $52 \%-79 \%$ and $65 \%-86 \%$ respectively).

\section{Objectives}

We aimed to compare performance and convenience of the new molecular Alere ${ }^{\mathrm{TM}} \mathrm{RSV}$ to our usual methods in routine conditions.

\section{Methods}

Consecutive clinical samples were collected during 15 days within the epidemic period and tested with BinaxNOW®, SimulFluor ${ }^{\circledR}$ and Alere $^{\mathrm{TM}} \mathrm{i}$ according to the manufacturer's instructions. In absence of PCR 
in our lab, we used a composite gold standard, with help of the National Influenza Reference Center (NRC) in order to confirm discordant results.

\section{Results}

Thirty-six paediatric and 13 adult samples were tested during the study period (January 9 to 24, 2017). Twelve out of 49 samples were found positive with the composite gold standard $(N=11$ in children 1y.o.; $N=1$ in a 83y.o. adult). We observed 6 discrepant results, positive with Alere ${ }^{\mathrm{TM}} \mathrm{i}$ and negative with both BinaxNOW® and SimulFluor ${ }^{\circledR}$. Out of these 6 samples sent to the NRC, 2 were positive for RSV-B in PCR, 3 were negative, and 1 was positive for Entero/Rhinovirus. BinaxNOW® and SimulFluor® showed similar results, with sensitivity of $83.3 \%$ and specificity of $100 \%$ (NPV: $94.9 \%$ ). Alere ${ }^{\mathrm{TM}_{\mathrm{i}}}$ sensitivity and specificity were $100 \%$ and $89.2 \%$ respectively, with a PPV of $75.0 \%$.

Conclusions

Alere ${ }^{\mathrm{TM}} \mathrm{i}$ lacks specificity compared to BinaxNOW@ and SimulFluor ${ }^{\circledR}$, but is a very sensitive and easy-to-use tool for rapid detection of RSV in less than 13 minutes. Further experience is needed on larger series.

\section{9}

\section{Infectious Diseases}

Prevention of parent to child transmission of HIV : An experience from Jammu, Jammu \& Kashmir, India

Sanjeev Kumar Digra

${ }^{T}$ Pediatrics, Government Medical College Jammu, India

\section{BACKGROUND}

Prevention of mother to child transmission of HIV is one of the important HIV prevention activities. Earlier short course of zidovudine and single dose of nevirapine were used for this pupose which has now been replaced by 6 weeks administration of nevirapine to newborns. It is a simple and low cost intervention to substantially reduce the risk of HIV transmission from mother to child. Nevirapine is favoured in resource constrained settings because it is cheap, easy to administer, rapidly absorbed and has a long half life.

\section{OBJECTIVES}

To assess the prevention of parent to child transmission of HIV in a tertiary care hospital in Jammu.

\section{RESULTS}

Over a period of 5 years w.e.f 1st March 2012 to 28th February 2017 a total of 18 babies were born to mothers with HIV in SMGS hospital, Government Medical College Jammu. Two babies received zidovudine for 6 weeks and 16 received nevirapine for 6 weeks soon after birth as preventive measure. Ten out of 18 were males. Majority (12) were from rural areas of Jammu.None of them was initiated on breast feeding. In two cases father was negative for HIV. In 9 cases ART was started in mother during 1 st trimester while in 5 and 4 it was started in 2 nd and 3 rd trimester respectively. At the end of study on 28th February 2017 youngest subject was 1 day old and the eldest was 4.5 years old. Five children who were more than 18 months of age at the end of study tested negative to HIV. All remaining subjects were asymptomatic.

\section{CONCLUSION}

Short term (6 weeks) administration of zidovudine or nevirapine to newborns and avoiding breast feeding resulted in prevention of mother to child transmission of HIV.

\section{2}

Infectious Diseases

Co-Administration of Trimethoprim-Sulfamethoxazole during HighDose Methotrexate - Haematologic Side Effects?

Ulrike Wanz ${ }^{I}$, Herwig Lackner ${ }^{l}$, Wolfgang Schwinger ${ }^{I}$, Martin Benesch ${ }^{l}$, $\overline{\text { Petra Sovinz }}^{I}$, Daniela Sperl ${ }^{I}$, Markus G Seidel ${ }^{I}$, Anna Karastaneva ${ }^{I}$, Christian Urban ${ }^{I}$, Volker Strenger ${ }^{1}$

${ }^{1}$ Department of Paediatrics and Adolescent Medicine, Medical University Graz, Austria

\section{Introduction}

While High-dose Methotrexate (HDMTX) is part of several chemotherapeutic regimens, Trimethroprim-Sulfamethoxazole (TMP/SMX) is commonly used to prevent Pneumocysits-jiroveci pneumonia, in immunocompromised patients. Since these drugs are folate antagonists, coadministration is not recommended to avoid cumulative toxicity. We explored haematologic effects of co-administration of HDMTX and TMP/ SMX.

Methods

Consolidation therapy for acute lymphoblastic leukaemia include four courses of HDMTX $\left(5000 \mathrm{mg} / \mathrm{m}^{2} / \mathrm{d}\right.$ intravenously) together with daily 6Mercaptopurine ( $50 \mathrm{mg} / \mathrm{m}^{2}$ orally). For Pneumocystis-pneumonia-prophylaxis, patients receive either TMP/SMX $5 \mathrm{mg} / \mathrm{kg} / \mathrm{d}$ (based on TMP component) p.o. for 3 or 4 days weekly or Pentamidine $300 \mathrm{mg}$ by inhalation once monthly.

We retrospectively compared blood count parameters before and 14 days after HDMTX-administration (Wilcoxon-Test) as well as relative changes of these parameters between patients with and without TMP/SMX prophylaxis (Mann-Whitney-U-Test).

\section{Results}

We analyzed 112 HDMTX-episodes in 28 patients (1.4 to 19.1; median 5.4 years old; $42.9 \%$ female.).

Compared to baseline parameters at HDMTX-administration, 14 days thereafter we observed widely spread changes of the white blood count (WBC, -78.4 to +251.8 ; median $-6.7 \%$ ), absolute neutrophil count (ANC, -85.5 to +406.2 ; median $-12.6 \%$ ), lymphocytes (Ly, -94.8 to +161.4 ; median $-5.0 \%$ ), thrombocytes ( $\mathrm{Thr},-78.5$ to +173.2 ; median $-13.2 \%$ ) and haemoglobin $(\mathrm{Hb},-32.3$ to +44.1 ; median $+3.8 \%)$. The WBC $(p=0.003)$, ANC $(p=0.029)$ and $\mathrm{Thr}(\mathrm{p} 0.001)$ were significant lower and $\mathrm{Hb}(0.001)$ was significant higher as prior to HDMTX-administration. Difference of lymphocytes was not significant.

Comparing episodes with TMP/SMX-prophylaxis $(n=51)$ and with Pentamidine-prophylaxis $(n=61)$, relative changes showed no significant differences for any of the tested parameters.

Discussion

While most parameters decreased after HDMTX (additionally caused by 6-Mercaptopurine co-administration), co-administration of TMP/SMX seems to have no significant influence on these changes.

Thus, our results do not support the recommendation to avoid coadministration of HDMTX and TMP/SMX

\section{0}

Infectious Diseases

Reference-controlled study of Coryzalia medication efficacy in children with acute infectious (IR) and obstinate allergic rhinitis (AR). Irina Farber ${ }^{1}$, Natalya Geppe ${ }^{l}$

${ }^{T}$ Departament of child diseases, I.M. Sechenov First Moscow State Medical University, Russia 


\section{Aim}

Study of comparative efficacy of Coryzalia in children with IR and AR. The study included 74 children from 3 to 15 years $(8.4 \pm 2.5)$. The patients were divided into 3 groups and 4 subgroups -34 with IR (1), 30 with AR (2), 10 with IR + AR (3). $1 \mathrm{~A}$ - monotherapy-16 children with IR, 1B-combined with vasoconstictive medication - 18 with IR; 2A monotherapy- 15 with $\mathrm{AR}, 2 \mathrm{~B}$ - combined with antihistamines -15 with AR. Coryzalia was prescribed in 1 tablet every 1 or 2 hour during the day. The treatment lasted from 5 (IR) to 14 (AR) days.

We also estimated allergic anamnesis, computerized bronchophonography (CDPhG), ciliary potency: saccharin test, brush biopsy. Nasal stuffiness during night and day, nasal cavity discharge, sneezing, cough were estimated from 0 to 4 points.

\section{Results}

All clinical symptoms are improvement in $82 \%$ of patients with IR, $67 \%$ with AR, $50 \%$ with IR and AR. In therapy IR there was improvement of $\mathrm{CDPhG}$ in a low frequency range in group 1A from $120.1 \pm 10.5$ to $113.4 \pm 19.29(\mathrm{p} 0.05)$; and in 1B from $90.6 \pm 30.5$ to $66.55 \pm 10.4$ (p0.001); as long as in a high frequency range in $1 \mathrm{~A}$ from $0.15 \pm 0.23$ to $0.08 \pm 0.03$ (p0.05); in $1 \mathrm{~B}$ from $0.16 \pm 0.2$ to $0.1 \pm 0.1$ (p0.03). In therapy of $\mathrm{AR}$ in $2 \mathrm{~A}$ from $147.8 \pm 12.5$ to $116.6 \pm 9.29(\mathrm{p} 0.001)$; in $2 \mathrm{~B}$ from $200.7 \pm 10.5$ to $105.6 \pm 8.11$ (p0.015); in a high frequency range in $2 \mathrm{~A}$ from $0.22 \pm 0.23$ to $0.15 \pm 0.03(\mathrm{p} 0.05)$; in $2 \mathrm{~B}$ from $0.34 \pm 0.23$ to $0.196 \pm 0.03(\mathrm{p} 0.01)$. In children with IR the time of saccharin test was reduced from $22.33 \pm 12.1$ to $12.5 \pm 7.4$ min (normally $8.8 \pm 4.9$ ); with AR from $19.0 \pm 11.2 \mathrm{~min}$ to $14.1 \pm 7,3$.

$\mathrm{CDPhG}$ can be used to cure both IR and AR. Coryzalia improves clinical effect of treatment and quality of life of patients.

\section{8}

\section{Infectious Diseases}

Case report: faces of multiple respiratory tract infections Justina Germanaviciene ${ }^{I}$, Valdone Miseviciene ${ }^{I}$, Vestina Martinkute ${ }^{2}$ ${ }^{T}$ Pediatric clinic, department of pulmonary diseases, Hospital of Lithuanian University of Health Sciences, Kauno Klinikos, Lithuania

${ }^{2}$ Pathological anatomy clinic, department of cytology, Hospital of Lithuanian University of Health Sciences, Kauno Klinikos, Lithuania

\section{Background}

Atypical and combined respiratory infections can present with different clinical symptoms and mimic various diseases. Unfortunately the diagnosis is sometimes difficult and usually retrospective.

\section{Aim}

To present case report about complex of various clinical symptoms and rare brochoscopic changes caused by combined respiratory infection.

\section{Methods}

Retrospective analysis of the patient's medical data.

\section{Results}

11 y old boy was admitted to the hospital due to severe hyponatremia, low grade fever, mild diarrhea and cough. Lung auscultation was normal, later crackles on the right side appeared. Chest X-ray showed infiltration on the right, blood test was normal. Suspected pneumonia was treated with Penicillin following with Clarithromycin. On the 9th day changes on the X-ray decreased, IgM Mycoplasma and Chlamydia pneumonia were negative, the boy was free of symptoms therefore he was discharged from the Clinic. Four days later the patient returned back because of high fever, dry cough and moderate diarrhea. Crackles on the right side were detected again, mild bronchial obstruction appeared. However, blood test, Chest $\mathrm{X}$-ray were normal, spirometry showed mild partially reversible bronchial obstruction. Symptomatic treatment was not effective. Bronchoscopy showed infiltration of the right bronchus with soft multifocal nodules (Fig. 1) without clear origin. Lymphocytes with some alveolar macrophages (Fig. 2) were present in the cytology. Paired sera for Mycoplasma pneumoniae was positive and Boca virus was identified from the nasopharyngeal swab. Azithromycin was given because of persistent respiratory symptoms and confirmed atypical infection. After ten days repeated bronchoscopy was normal (Fig. 3), the patient was clear of all symptoms.

\section{Conclusion}

Human Boca virus was the leading pathogen of the whole clinical picture in our patient. Co-infection with Mycoplasma pneumonia stimulated more severe and complicated disease. Suspition and identification of possible infection on time is very impotant for further clinical decisions.

Fig. 1

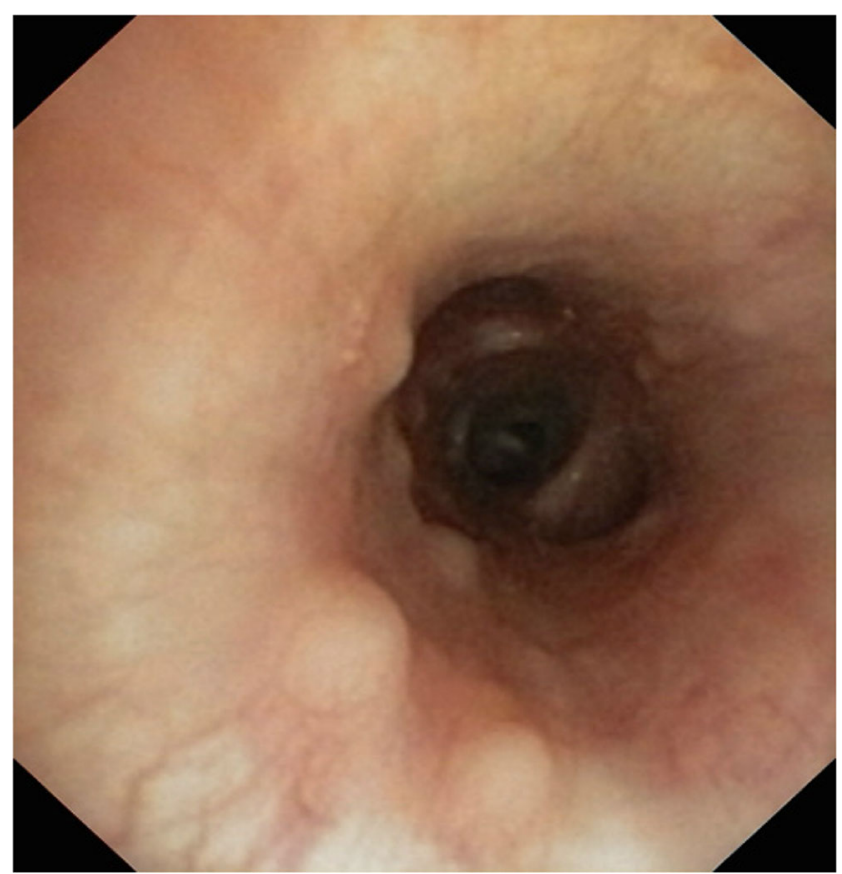

Fig. 2

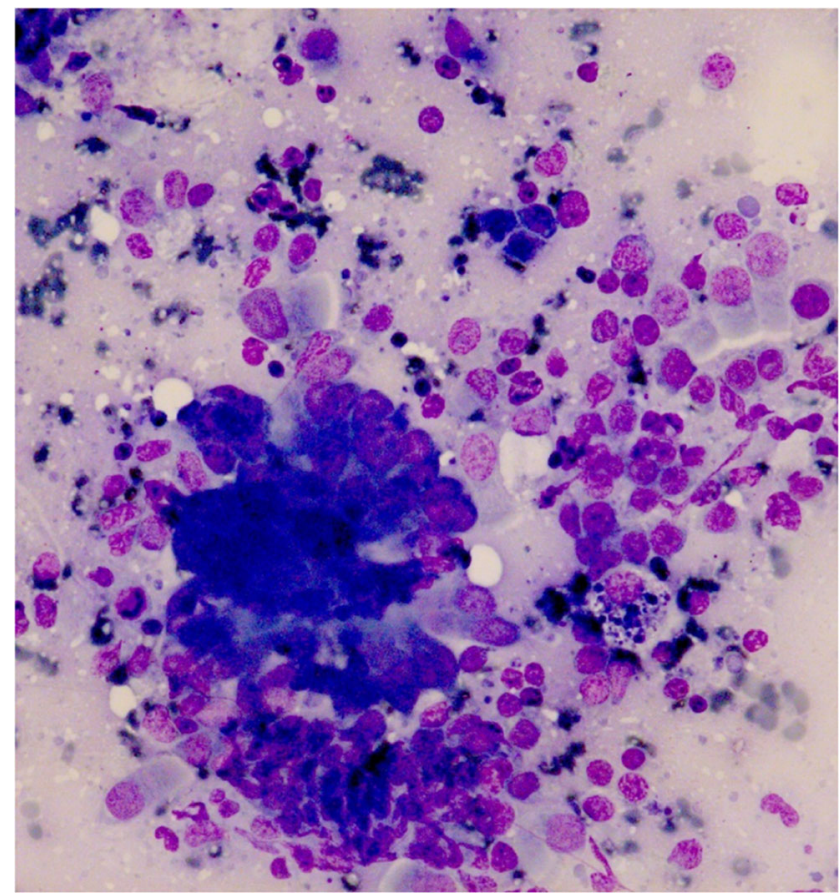


Fig. 3

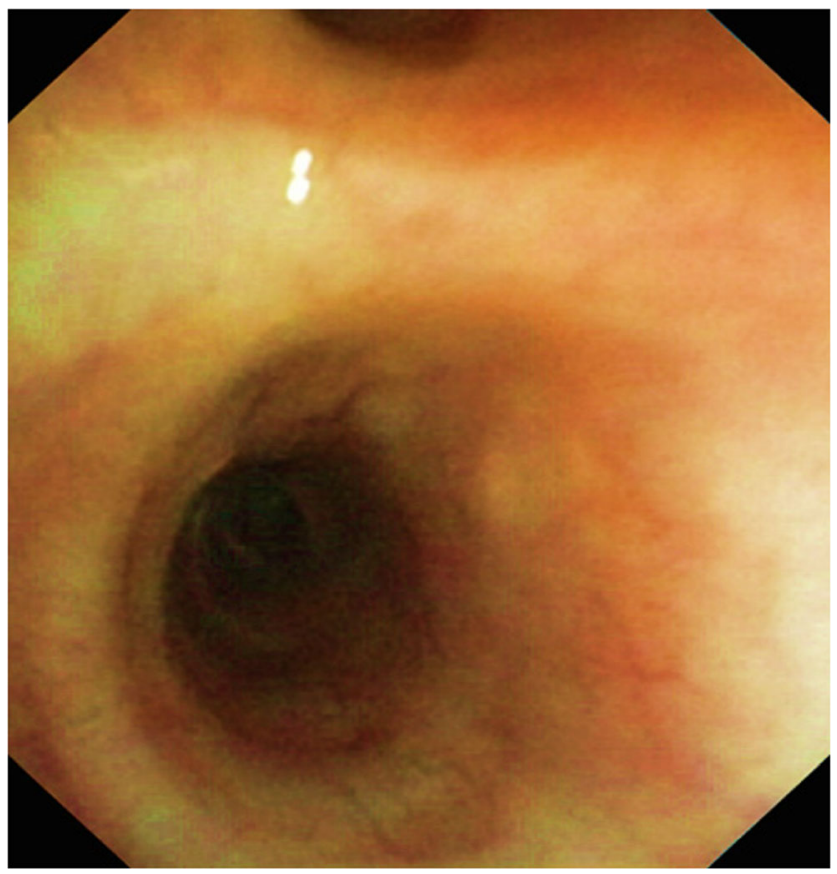

511

\section{Infectious Diseases}

An investigation to Improve the Effectiveness of the Use of Real-time Multiplex PCR Testing for Viral Respiratory Infections James Gray ${ }^{I}$, Shona Braybrook ${ }^{1}$

${ }^{I}$ Department of Microbiology, Birmingham Chiildren's Hospital, UK

\section{Background}

Multiplex PCR tests are increasingly used to accurately and rapidly diagnose infectious diseases, including viral respiratory infections (VRI). These tests can assist in addressing clinical challenges such as infection control, antimicrobial stewardship and bed pressures. Optimal use of these tests depends on streamlining all stages of the diagnostic pathway, from sample collection to results reporting. However, often new tests are simply introduced into existing diagnostic pathways that are not designed to provide full advantage from these new, expensive tests. Objective

To investigate the current use of multiplex PCR testing for VRI and to develop a toolkit to improve practice throughout whole diagnostic pathway. Methods

VRI testing by PCR was introduced on a $24 / 7$ basis at Birmingham Children's Hospital (BCH) in autumn 2015. Retrospective review of VRI testing on 3 priority wards (PICU, Paediatric Assessment Unit, Haematology/Oncology) during the 2015-2016 winter. We then undertook a questionnaire survey of clinical staff to determine their understanding and experiences of the diagnostic pathway in autumn 2016.

\section{Results}

The 85 tests reviewed took on average twelve hours and seven minutes from sample taken to result availability. Delays were identified during specimen transport to the laboratories, but particularly during laboratory handling. 36/85 samples gave negative results; RSV and rhinoviruses were the commonest viruses detected (16 cases of each). Ward questionnaires indicated that staff considered that infection control isolation management the main reason for testing; the majority of respondents also believed that antibiotic treatment would be influenced in at least $25 \%$ of cases.

\section{Conclusion}

The testing protocol was inefficient and overly complex; a diagnostic test that took one hour to perform was taking 12 hours to generate results.
Opportunities for better clinical management were consequently missed. A redesign of the entire diagnostic pathway is now underway.

\section{4}

\section{Infectious Diseases}

Epidemiology of Lyme Borreliosis among Risk-Group Children of Western Ukraine

Halyna Pavlyshyn ${ }^{1}$, Nataliya Haliyash ${ }^{1}$, Mariia Shkilna ${ }^{2}$, Ivanna Horishna ${ }^{l}$, Viktoriya Furdela ${ }^{I}$

${ }^{I}$ Department of Pediatrics, I.Horbachevsky Ternopil State Medical University, Ukraine

${ }^{2}$ Department of Infectious Diseases and Epidemiology, Dermatology and Venereology, I.Horbachevsky Ternopil State Medical University, Ukraine

\section{Background}

Western Ukraine, including Ternopil region, is an endemic area for Lyme borreliosis, as it is located in a zone with fertile soils, moderate continental climate, and forest landscapes. It provides maintenance of the main tick vectors of Borrelia burgdorferi sensu lato - Ixodes ricinus ticks in the wild. Objective

To detect the prevalence of Lyme borreliosis among the pediatric population of Ternopil region, Western Ukraine.

\section{Methods}

The group of 108 children, members of the Youth Scout Organization 'PLAST', was observed because of the extremely high risk of exposure to the Lyme borreliosis. All subjects have answered the question of compatible international questionnaire, in that marked: amount and places of the Ixodes ricinus tick bites; methods of removing of the ticks from the body surface; inspection of the ticks for the causative agent of Lyme borreliosis; the most frequent symptoms of the diseases, which appeared after the tick bites; using of the repellents during stay in the forest; checking the body surface for the presence of ticks after return from the forest.

\section{Results}

The results of questionnaire study indicate that 85 (78.7\%) of children, aged $(13.1 \pm 2.7)$ years, reported to have tick bites. $59(54.6 \%)$ experienced tick bites while staying in the forest camps. 40 (37.04\%) children had multiple tick bites. The ticks were removed during the first $24 \mathrm{~h}$ in 72 $(66.7 \%)$ of them. Erythema migrans was observed in $12(11.1 \%)$ scouts. The screening test Elisa (Euroimmun) has been conducted in 16 (14.8\%) study participants to detect the presence of antibodies IgM/IgG antiBorrelia burgdorferi sensu lato. The further investigations are planned.

\section{Conclusion}

The elaboration of educational, preventive and efficacy monitoring standards for the residents of the area (children) is required. A two-step algorithm consisting of ELISA and Western blot tests should be used for this group. Children with tick bites should receive preventive treatment.

\section{0}

\section{Infectious Diseases}

\section{EXTRAINTESTINAL MANIFESTATIONS IN CHILDREN WITH} VIRAL DIARRHEA

Dumitra Simona ${ }^{1}$, Pilat Luminita ${ }^{2}$, Crisan Carmen ${ }^{1}$, Horga Adeline $\frac{\text { Larisa }}{}^{1}$, Covaci Claudial, Balan Ozana ${ }^{1}$, Stanculet Carmen $^{I}$, Pop

\section{${ }^{1}$ PEDIATRICS, Vasile Goldis Western University of Arad, Romania}

${ }^{2}$ PEDIATRICS, Arad County Emergency Clinical Hospital, Romania

\section{Introduction}

There is a wide and complex spectrum of extraintestinal clinical manifestations at children with gastroenteritis, most often hiding the severity and intensity of digestive signs. The purpose was to emphasize the main extraintestinal manifestations, to evaluate the presence of some specific signs of the Rotavirus/Norovirus infection. 


\section{Material and method}

We evaluated 124 children with viral diarrhea, confirmed by ELISA Ridascreen Rotavirus and Norovirus tests, from October 2014 to January 2016 in the Pediatrics Clinic from Arad. The group included two subgroups: subgroup 1-93 children with Rotavirus infection,subgroup 2 - 31 children with Norovirus infection. We have monitored several clinical parameters and we have compared the frequency of the symptoms, to find statistical elements which are suggestive of the etiology. The data was analyzed by Stata v12 statistical analysis program, using the Student $t$ and Z-test.

Results and discussion

Agitation was found in $37.1 \%$ of cases, lethargy in $29.8 \%$, seizures in $4 \%$, rhinorrhea in $17.7 \%$, myalgia in $13.7 \%$, chills at $5.6 \%$, cough in $20.2 \%$ cases.Compared to group Rotavirus/Norovirus - agitation appeared in $32.3 \% / 51.6 \%(p=0.05)$, lethargy in $30.1 \% / 29 \%$ $(p=0.90)$, seizures in $4.3 \% / 3,2 \%(p=0.79)$, rhinorrhea in $12.9 \% /$ $32.3 \%(p=0.01)$, myalgia in $17.2 \% / 3.2 \%(p=0.05)$, chills $7,5 \% / 0 \%$ $(p=0.11)$, cough in $16.10 \% / 32.3 \%(p=0.05)$.

\section{Conclusion}

The main extraintestinal manifestations in children with viral diarrhea are the changes in behaviour (agitation, lethargy) and cough. Rhinorrhea was found in the Norovirus group rather than the Rotavirus group.

Key words: diarrhea, Rotavirus, Norovirus, extraintestinal manifestations

\section{9}

\section{Infectious Diseases}

Association of ADAMTS-13 Activity with Clinical Outcomes in Hospitalized Children with Infections

Irina Katirlo $^{1,4}$, Alina Prokofjeva ${ }^{1,4}$, Anna N̦ikulcenkova ${ }^{1,4}$, Gita $\overline{\text { Krievina }}^{3}$, Péteris Tretjakovs ${ }^{3}$, Ilze Grope ${ }^{1,4}$, Reinis Balmaks ${ }^{2,4}$, Dace Gardovska ${ }^{1,4}$

${ }^{1}$ Department of Paediatrics, Riga Stradins University, Latvia

${ }^{2}$ Department of Clinical Skills and Medical Technology, Riga Stradins University, Latvia

${ }^{3}$ Department of Human Physiology and Biochemistry, Riga Stradins University, Latvia

${ }^{4}$ Clinic of Children's Diseases, Children's Clinical University Hospital, Latvia

\section{Background}

Deficient proteolysis of ultra-large von Willebrand factor multimere caused by decreased activity of ADAMTS-13 may result in thrombotic microangiopathy, which is one of the organ damage mechanisms in septic patients. Objective

The aim of this work was to analyze ADAMTS-13 blood concentration in association with clinical disease characteristics and outcomes in hospitalized children.

\section{Methods}

The study was conducted in a tertiary children's hospital in Latvia. Children aged from 1 day to 18 years hospitalized with infections of various localization were enrolled prospectively during the period from February 2015 to January 2017. Complete blood count, C-reactive protein (CRP), interleukin-6 (IL-6), and procalcitonin (PCT) were performed using standardized methods. ADAMTS-13 was determined by Quantikine ELISA Human ADAMTS-13 Immunoassay test.

\section{Results}

During the study period, 77 patients were enrolled - $31(40.3 \%)$ girls and 46 (59.7\%) boys with average age of 52 months (interquartile range 16.5-87.5). Median ADAMTS-13 concentration was $693.38 \mathrm{ng} / \mathrm{ml}$ (interquartile range 584.75-770.37). Diagnosis of sepsis was established in 51 (66.2\%) children and the mean ADAMTS-13 concentration was significantly lower in these patients (mean difference $110.08 \mathrm{ng} / \mathrm{ml}$; $95 \%$ CI 51.77-168.37; p0.001). Sepsis was defined as severe infection with life-threatening organ dysfunction. Decreased ADAMTS-13 concentrations correlated with increased CRP $(r=-0.323 ; p=0.004)$, IL-6 $(r=-0.226 ; p=0.049)$, length of stay in hospital $(r=-0.425 ; \mathrm{p} 0.001)$ and in paediatric intensive care unit $(r=-0.238 ; p=0.037)$, and a tendency to longer mechanical ventilation in intubated patients $(r=-0.217 ; p=0.058)$. The mean ADAMTS-13 concentration was significantly lower in paediatric intensive care unit patients, compared to patients hospitalized on general paediatric wards (mean difference $159.04 \mathrm{ng} / \mathrm{ml} ; 95 \%$ CI 69.29-248.8; $p=0.001)$.

Conclusion

Decreased ADAMTS-13 concentration was associated with more pronounced inflammation and sepsis, poorer clinical outcomes in hospitalized children with infection. It makes this protein interesting as a potential diagnostic marker of the organ damage.

\section{5}

Infectious Diseases

\section{NEONATAL MENINGOCOCCAL MENINGITIS} COMPLICATED BY CEREBRAL SINOVENOUS THROMBOSIS Kene Maduemem ${ }^{1}$, Muhammad Rizwan ${ }^{1}$, Comfort Adedokun ${ }^{2}$

${ }^{T}$ Paediatrics, Cork University Hospital, Ireland

${ }^{2}$ Emergency Medicine, Cork University Hospital, Ireland

\section{BACKGROUND/OBJECTIVE}

Neisseria meningitidis is one of the major causes of meningitis in children and adolescents, but it is rarely found during the neonatal period. The outcome of meningococcal disease can be fatal and depends on the innate immune system, age, serogroups, pre-existing antibodies, and other unknown host factors. We report a case of an eight day old with serogroup B meningococcal meningitis complicated by cerebral sinovenous thrombosis (CSVT).

\section{METHODS}

Clinical data, laboratory and radiological records of our case were reviewed. RESULTS

An eight day old male neonate presented with fever, poor feeding and subtle seizures. Neisseria meningitidis serogroup B was isolated in blood and cerebrospinal fluid by viral polymerase chain reaction. Magnetic resonance venography (MRV) of the brain demonstrated sagittal sinus thrombosis. He was managed on twelve week course of daily subcutaneous tinzaprin (low molecular weight heparin). Follow up MRVs confirmed re-canalization of the vessel after anticoagulant therapy. He made a remarkable recovery and was followed up till one year of age with no neurological sequale.

\section{CONCLUSION}

To the best of our knowledge, this is the first documented case of neonatal meningococcal meningitis complicated by CSVT in Republic of Ireland. It is a rare but fatal complication which can be managed if detected early. Keywords: neonatal meningitis, cerebral venous thrombosis, serogroup $\mathrm{B}$, tinzaparin

\section{3}

\section{Infectious Diseases}

The seroprevalence of HHV-7 in children in Russia Mikhail Nikolskiy ${ }^{1}$, Dmitriy Lioznov ${ }^{2}$

${ }^{T}$ Pediatrics, Pavlov First State Medical University, Russia

${ }^{2}$ Infectious Diseases, Pavlov First State Medical University, Russia

\section{Background}

There are no data on HHV-7 seroprevalence in Russia. Previously we detected HHV-7 DNA in plasma of 8-10\% children with exanthem subitum and fever without rash.

\section{Objective}

We aimed to comparative study of the seroprevalence of HHV-7 and HHV-6 in children in Russia.

\section{Methods}

The pilot study included 105 children, aged from 10 days to 10 years, and diagnosed with respiratory tract infections. All children were recruited at St.Petersburg Filatov's Children Hospital between March-April 2016. 
Serum samples were tested for IgG to HHV-7 and HHV-6 by indirect immunofluorescent assays and ELISA correspondingly. We distributed patients into five groups, according to their ages: under six months, from six months to 1 year, from one year to 2 years, 2-4 years, and older than 4 years.

\section{Results}

Antibody prevalence for HHV-7 was three times lower than HHV-6. There were only $16 \%$ patients seropositive to HHV-7, compared to $52 \%$ seropositive to HHV-6. Under 6 months old the frequency of HHV-7 seropositive was $14.8 \%$, which possible was due to maternal antibodies. From 6 months to 4 years the HHV-7 seroprevalence was about 8-13\%. In children older than 4 years the rate of seropositivity rised to $28 \%$.

\section{Conclusion}

We can conclude that contact to HHV-7 occurs later in life than to HHV-6, predominantly after age of four years. The seroprevalence to HHV-7 is lower than to HHV-6 in children under 10 years. The sample size of our study is too small to extrapolate on Russian population and other seroprevalence studies are needed.

301

\section{Infectious Diseases}

Risk of Lyme Borreliosis in children of Ternopil region

Svitlana Nykytyuk ${ }^{1}$, Mariia Shkilna ${ }^{2}$, Kateryna Yavorska ${ }^{2}$, Kateryna Vorobets $^{2}$, Oksana Chybata

${ }^{1}$ Department of Pediatrics, I. Horbachevsky Ternopil State Medical University, Ukraine, Ukraine

${ }^{2}$ Department of Infectious Diseases and Epidemiology, Dermatology and Venereology,, I. Horbachevsky Ternopil State Medical University, Ukraine, Ukraine

${ }^{3}$ Infection -diagnostic, Ternopil Regional Children Hospital, Ukraine

\section{Background}

Lyme disease - natural and nucleus inoculable disease caused by Borrelia(Borreliaburgdorferi), catching by clips and becomes apparent by inoculable ring-shaped erythema, fever, affection of central and peripheral neural system, heart and big joints. This disease occurs on the territory of Eurasia of forest and forest-steppe zones.The western part of Ukraine including Ternopil oblast is endemic zone of Lyme borreliosis. It is located in an area with the forest landscapes (the total area of forest area is 199.3 thousand ha of broad leaves and mixed-broad leaves trees pecies); Areas Ixodestick-borneborreliosis found in 57 localities 14 districts of the region and the city of Ternopil.

\section{Objective}

To detect the prevalence of Lyme borreliosis among children of Ternopil region, Western Ukraine.This study is a part of the Ukrainian-Polish project "Investigation of epidemiology, pathogenesis, clinics and prophylaxis of borreliosis"

\section{Methods}

These studies covered 200 children, with middle age (10.02 \pm 4.29$)$.There were patients from Ternopil regional children hospital with different somatic pathology. The croup of the examined patients comprised $44 \%$ girls and $56 \%$ boys. Thirty-two of the tested group, has answered the questions of compatible international questionnaire.

\section{Results}

The majority of the examined children $(47.0 \%)$ reported tick attack during their life. There were children from the early school age. On seasonal, tick bites the highest rate was during May-July, where in May $-6 \%$, in June $-38 \%$, in July $-31 \%$, in August $-3 \%$, in September $6 \%$ accordingly. The most disturbing is that $6 \%$ of examined patients do not remember the month of the tick bite generally.

\section{Conclusions}

The presented study show, that children's from Ternopil region (endemic zone of Lyme borreliosis), are exposed to tick bite, more often in May-July of the year.The elaboration of educational and prophylactic standards among the tested group is needed, more active during the winter months.

\section{9}

\section{Infectious Diseases}

Prevalence of Mastoiditis in Children Hospitalized in the Last 16 Years in a Teaching Hospital Alcides Carneiro - Rio de Janeiro - Brazil Eneida Quadrio de Oliveira Veiga ${ }^{l}$, Barbara Pires Martins ${ }^{l}$, Felipe Freitas Andrade $^{I}$, Lucas Mello Netto ${ }^{I}$, Nathalia Veiga Moliterno ${ }^{l}$, Felipe Machado Moliterno ${ }^{1}$, Susie Andries Nogueira ${ }^{l}$, Solimar Stumpf Cordeiro ${ }^{1}$, Alvaro Veiga ${ }^{1}$

${ }^{1}$ Pediatrics, Faculty of Medicine of Petrópolis, Brazil

\section{Background}

Acute Mastoiditis is defined as inflammation/infection of the aerated cells of the mastoid process of the temporal bone, being the most frequent evolutionary course of Acute Otitis Media. The pediatric age group is particularly more susceptible to the sequel and complications inherent to the course of this pathology. According to the literature, its prevalence in the last years remains stable, the average age is below 4 years, there is predilection for the male sex and complications are associated with high leukocyte count and high values of C-reactive protein (CRP).

\section{Objective}

To identify common characteristics in children hospitalized HEAC for Acute Mastoiditis in the period 2001-2017.

\section{Methods}

Retrospective, cross-sectional, quantitative, descriptive study of Acute Mastoiditis cases occurred in the last 16 years in HEAC Pediatrics. Variables analyzed: sex, age, hospital stay, comorbidities, complications, therapeutics, complementary exams and outcome.

\section{Results}

Total hospitalizations during the study period: 17,058. Hospitalizations for acute mastoiditis: $24(0.14 \%)$. Averages: Age 5.1 years; Length of stay: 14 days; Antibiotic therapy: 25 days. Complications: 6 (50\% tympanic perforation); $100 \%$ initial diagnosis of Otitis Media. Leukocytosis $71.42 \%$ and increased CRP in $64.2 \%$. Surgical approach: 1 (7.4\%). Initially the most used empirical antibiotic therapy: Ceftriaxone $(21.4 \%)$ and Ceftriaxone + Oxacillin $(21.4 \%)$. Terminal monotherapy: Ceftriaxone in $28.57 \%$. Antimicrobial therapy maintained at $42.85 \%$, followed by completion with Amoxicillin + Clavulanate $21.4 \%$. Computed tomography: $71.42 \%$. Good evolution: $95.83 \%$.

\section{Conclusion}

In contrast to the world literature, the majority of cases progressed satisfactorily with the use of antibiotic monotherapy, only one case required surgical resolution, we did not find gender preference and the mean age was higher than expected. Although we find low prevalence of complications and inadequate outcome, it is necessary to strictly comply with the diagnostic and therapeutic protocols of this and other pathologies in order to minimize sequel and serious complications for the future of our small patients.

\section{7}

\section{Infectious Diseases}

"Achievements" Claimed In The EMA Paediatric 10-Years-Report On The EU Pediatric Regulation Don't Withstand Critical Examination Klaus Rose

${ }^{T}$ Medical Science, klausrose Consulting, Switzerland

\section{Background}

The EMA 10-years-report claims that many medicines for the treatment of infectious diseases became available after completion of paediatric investigation plans (PIPs), giving peginterferon alpha (PEG $\alpha$ ) for hepatitis $\mathrm{C}$ as an example.

\section{Objective}

To verify/challenge the EMA's claims and the medical justifications given for requiring separate safety \& efficacy (S\&E) studies in minors. 


\section{Methods}

PIPs listed on the EMA website and associated publications of pediatric $\mathrm{PEG} \alpha$ trials were analyzed for medical validity considering basic principles of developmental pharmacology/physiology and commonsense.

\section{Results}

Three PIPs required pediatric hepatitis C S\&E studies: two open-label studies PEG $\alpha$ + ribavirin in 3-17 year olds, and one double-blind placebo-controlled study in 5-17 year olds. The latter, performed in the USA (Schwarz K et al. 2011), confirmed superiority of PEG $\alpha+$ ribavirin over $\operatorname{PEG} \alpha$ alone shown years before in adults. The belief that children need separate proof of S\&E originates from the poorly supported dogma that "kinetics, end organ responses, and toxicities" of drugs in children of all ages differ fundamentally from adults, expressed in key documents of the American Academy of Pediatrics (AAP). This dogma wrongly uses "children" as both a legal and physiological term. Children are not another species. Physiology and pharmacology often differs in babies and young children but not in most, more mature minors.

The EMA's claim that their PIPs made PEG $\alpha$ available for children with hepatitis $\mathrm{C}$ is misleading at best. PEG $\alpha$ was available off-label without PIPs. Medically, assessment of paediatric dosing is needed, but this can be better achieved by modeling \& simulation and confirmation by opportunistic PK/PD studies rather than the medically and ethically questionable, PIP-demanded PEG $\alpha$ double-blind placebo-controlled S\&E study.

\section{Conclusion}

The EAP should voice an independent position on what type of paediatric regulatory studies are needed based on science, developmental pharmacology/physiology, and commonsense; not on dogma.

\section{6}

\section{Infectious Diseases}

Uptake of Pertussis and Influenza Vaccinations in Pregnancy Anja Saso $^{1,2}$, Beverly Donaldson ${ }^{1}$, Beate Kampmann ${ }^{1}$

${ }^{T}$ Department of Paediatrics, Imperial College London, UK

${ }^{2}$ Department of Paediatrics, Barnet General Hospital, UK

\section{Background}

Maternal immunisation may be a key strategy in reducing neonatal morbidity and mortality worldwide. In the UK, it is recommended that pregnant women receive one dose of pertussis-containing vaccine from 16weeks gestation in addition to annual influenza immunisation. Vaccine uptake remains poor, however, with considerable variation nationally.

\section{Objectives}

Our audit aimed to evaluate vaccine uptake at Barnet Hospital, London and explore factors influencing pregnant women's decisions to accept or decline immunisation.

\section{Methods}

A cross-sectional survey was conducted on 100 women over 18years old on the postnatal ward in May 2016. A short, anonymised questionnaire was designed to collect data on patient demographics, vaccine status, awareness of recommended vaccinations, sources of information, and reasons for accepting or declining immunisation. Answers were cross-checked with patient notes (if recorded) to account for inaccuracy in self-reported vaccine status. Quantitative data was tabulated into Microsoft Excel and descriptive statistics calculated. Qualitative data from free text was manually analysed and divided into key common themes.

\section{Results}

Of the 100 participants, $32 \%$ had been vaccinated against pertussis and $36 \%$ against influenza during pregnancy. Of the women who had not been immunised with either vaccine, $63 \%(38 / 60)$ were aware of either programme but had still refused. The primary sources of information were GPs and/or midwives. The greatest barriers to uptake were paucity of information, particularly regarding the proposed benefits, uncertainty about potential risks to the fetus and lack of encouragement from professionals involved in their antenatal care.

\section{Conclusions}

Uptake of both vaccines was suboptimal. A significant proportion of women were either unaware, insufficiently informed or felt unsupported in taking the vaccinations. Therefore, we have implemented a series of changes focussing on improving education of relevant healthcare professionals, in order to ultimately boost patient knowledge and acceptance. New staff teaching sessions have been introduced and written information distributed specifically targeting those involved in antenatal care.

\section{4}

\section{Infectious Diseases}

Clinical Profile of Osteomyelitis and Septic Arthritis in Children in India Ira Shah ${ }^{1}$, Sareena Ameen ${ }^{1}$, Rujuta Mehta ${ }^{2}$

${ }^{T}$ Pediatrics and Pediatric Infectious Diseases, $B J$ Wadia Hospital for Children, India

${ }^{2}$ Pediatric Orthopedics, B J Wadia Hospital for Children, India

\section{Aim}

To describe the clinical profile of children with pyogenic osteomyelitis(OM) and septic arthritis(SA).

\section{Methods}

This observational study was conducted from May 2014 to May 2016. All children diagnosed as pyogenic OM and/or SA were included in the study. Clinical features, immunodeficiency status, the organism isolated and their resistance patterns were evaluated. Factors associated at different age groups was analysed.

\section{Results}

Out of 70 children, 18(25.7\%) had tuberculous OM and were excluded from the study. The mean age of presentation was $3.5 \pm 4.2$ years. $9(17 \%)$ were neonates, 11(21\%) were between 1 month-1 year, 19(37\%) were between 1 to 5 years and $13(25 \%)$ children were 5 years of age. Male:female ratio was 36:16. 39(75\%) patients had lower limb involvement, $8(15 \%)$ patients had upper limb involvement, $4(7 \%)$ had multifocal involvement and only one patient had spinal involvement. Bacterial culture was positive in 39(75 \%) patients and MSSA grew in 21(54\%) patients, MRSA grew in 11(28\%), gram negative organisms grew in $4(10 \%)$ and candida species were isolated in $3(8 \%)$ patients. No patient was detected to have acquired or congenital immunodeficiency. 20(38\%) patients had OM of which $19(95 \%)$ were 1 year of age $(p=0.0007)$, $22(42 \%)$ had SA of which $16(72.7 \%)$ were

\section{Conclusion}

Staphylococcal aureus is the most common organism isolated with predominant affection in boys. Lower limbs are more commonly affected than upper limbs. OM is more common in children above 1 year of age whereas SA is more common in infants.

\section{5}

\section{Infectious Diseases}

Recurrent Late-Onset Neonatal Group B Streptococcal Disease Transmitted Through Breast Milk: A Case Report

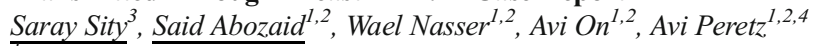

${ }^{T}$ Department of Pediatrics, Baruch Padeh Medical Center, Poriya, Tiberias, Israel

${ }^{2}$ Faculty of Medicine in the Galilee, Bar Ilan University, Zefat, Israel

${ }^{3}$ Sackler Faculty of Medicine, Tel-Aviv University, Israel

${ }^{4}$ Clinical Microbiology Laboratory, Baruch Padeh Medical Center, Poriya, Tiberias, Israel

\section{Background}

Group B Streptococcus is the most frequent cause of neonatal invasive disease. Recurrent GBS infections are infrequent, with reported incidences ranging from $1 \%$ to $6 \%$. Recurrent GBS infections usually represent persistent mucosal colonization, but occasionally reinfection occurs. Treatment for recurrent infection is suggested to be continued 
for one week longer than the usual course, but there are no data to indicate efficacy of this recommendation.

\section{Case presentation}

A healthy neonate, delivered by caesarian section who developed recurrent late onset GBS infection. Workup includes positive blood cultures. We cultured the breast milk of the mother and discovered GBS, the culture was verified twice. We had the mother stop breastfeeding and switch to formula. The neonate was treated with Ampicillin for 14 days and preventive Amoxicillin for 2 more months.

Discussion

Breast milk can be a route for neonatal contamination by pathogenic microorganisms. To the best of our knowledge, forty-eight cases of GBS late onset sepsis transmitted by breast milk were reported in the literature. Two main mechanisms of acquisition have been proposed. The first, based on recent studies in animal models suggest that bacteria from the maternal digestive tract may also colonize the breast. In the second, maternal milk ducts are generally assumed to be infected during breast feeding by bacteria that colonize the neonate's oropharyngeal mucosa in the perinatal period, the neonate is then re-infected as the concentration of bacteria increased in the breast milk. Conclusions

We suggest culturing breast milk for GBS in mothers whose children have recurrent late onset GBS infection.

\section{4}

\section{Infectious Diseases}

Is vaccination the end of the Rotavirus problem in Romania? Cristina Becheanu ${ }^{1,2}$, Alexandra Virginia Sobek ${ }^{1}$, Andreea Cristina Girbea $^{I}$, Iulia Florentina Tincu ${ }^{1,2}$, Ioana Oprescu ${ }^{I}$, Gabriela Lesanu ${ }^{1,2}$ ${ }^{1}$ Pediatric Gastroenterology, "Grigore Alexandrescu” Emergency Hospital for Children, Romania

${ }^{2}$ Pediatrics, Carol Davila University of Medicine and Pharmacy, Romania

\section{Background}

In Romania, although there is a Rotavirus vaccine available since 2007, viral acute gastroenteritis with this etiology is still a major cause of hospitalization in infants.

\section{Objective}

The aim of our study is to evaluate the trend of Rotavirus infection in hospitalized infants in 2016 vs. 2011.

Methods

We carried out an observational, retrospective study, which included all infants diagnosed with Rotavirus infection in "Grigore Alexandrescu" Emergency Hospital for Children from Bucharest, between January and December 2016. We compared our data with similar ones from a study conducted in the same conditions, during 2011.

\section{Results}

During 2016, 710 infants from the gastroenterology department were diagnosed with acute gastroenteritis, 177 with Rotavirus infection vs. 839 in 2011, of which 209 with Rotavirus. There is a significant decrease in the incidence of Rotavirus diarrhea in the past 5 years, $41.9 \%$ in 2011 vs. $24.9 \%$ in 2016 (p0.01). There were 93 cases $(24.9 \%)$ of nosocomial infection with Rotavirus reported in 2011, vs. 48 (24.85\%) in 2016. If in 2011 , the 3-6 months age group was most frequently affected by nosocomial Rotavirus ( $54.8 \%$ of cases vs. $29.2 \%$ in 2016 , p0.01), in the past year, the 6-12 months age group was mainly infected during hospitalization $(41.7 \%$ in 2016 vs. $21.5 \%$ in 2011, p0.01). The mean duration of hospitalization in 2011 was 6.3 days vs. 5.3 days in 2016 .

Conclusion

Although Rotavirus vaccination is not mandatory, the availability of the vaccine on the Romanian market determined a decrease in the incidence of acute gastroenteritis in infants, suggesting an improved strategy in informing the parents. Nosocomial infections with Rotavirus remain a significant problem, probably because of crowded hospitals. Remains exposed to nosocomial Rotavirus the 6-12 months age group, probably unvaccinated infants.
562

Infectious Diseases

Rotavirus Infection in Children: Burden and the Dominant Genotype in 2014-2015 years, Kiev, Ukraine

Mariia Teslenko $^{2}$, Lyudmila Chernyshova $^{1}$, Natalia Rodionova $^{3}$, Alexandr Kasyan

${ }^{T}$ Pediatrics Infection and Immunology Department, Shupyk National Medical Academy of Postgraduate Education, Ukraine

${ }^{2}$ Pediatric Infection and Immunology Department, Pediatric Infection Department, Shupyk National Medical Academy of Postgraduate Education, Pediatric Clinical Hospital \#1., Ukraine

${ }^{3}$ Pediatric Infection and Immunology Department, Pediatric Infection Department, Shupyk National Medical Academy of Postgraduate Education. Pediatric Clinical Hospital \#1, Ukraine

${ }^{4}$ Pediatric Infection Department, Pediatric Clinical Hospital \#1, Ukraine

\section{Background}

Rotavirus (RV) is the most common cause of severe gastroenteritis (GE) in infants and young children worldwide. There is no reliable epidemiological information on RV gastroenteritis (RVGE) in Ukraine. Rotavirus infection (RVI) is a vaccine preventable. To introduce universal vaccination, data on the burden of RVI and the dominant genotype must be collected.

\section{Objective}

To estimate the disease burden of RVGE and assess the relationship between the RV dominant genotype and the severity of clinical manifestations in Ukraine children up to 5 years old.

\section{Methods}

Hospital surveillance was conducted by using a WHO standard protocol. We performed an observational hospital based study which was conducted at Pediatric hospital \#1 Kyiv, Ukraine during 2014-2015 years. The disease severity was estimated by score scale for clinical manifestations of RVGE assessment of Vesikari. Detection of RV antigen in feces was carried out by ELISA. RV genotyping was performed by RT-PCR.

\section{Results}

Among the 367 samples studied stool RVI was confirmed in 181 $(49.3 \%)$. Genotyping was performed in 123 positive samples.

The following genotypes were revealed: G1P[8]-12.2\%(15/123); G2P[4]-2.44 \%(3/123); G2P[8]-0.81 \%(1/123); G3P[8]-3.25 \%(4/123); G4P[8]-73.98 \% (91/123); G9P[8]-4.07 \%(5/123); G2[NT]$1.63 \%(2 / 123)$; NT[P8]-0.81 \%(1/123); NT[NT]- $0.81 \%(1 / 123)$. The mean number of points on a scale Vesikari: G1P[8]-15(3,04); G2P[4]12(1,41); G2P[8]-15; G3P[8]-13.25(1,48); G4P[8]-14.68(2,56); G9P[8]15.4(1,5); G2[NT]- 12(2); NT[P8]-13; NT[NT]-12. The number of points which is typical for moderate course met only with genotypes G1P[8]$6.67 \%(1 / 15)$ and G4P[8]-4.4\%(4/91).

\section{Conclusions}

RVI is the main cause of hospitalization in children

Burden of RVGE, according to data about circulating genotypes justify the introduction of vaccination in Ukraine.

Key words: children, rotavirus, burden, genotype.

\section{0}

\section{Infectious Diseases}

The high seroreversion of Human Immunodeficiency Virus (VIH) in vertically exposed infants who received all the care measures to reduce the mother-to-child transmission in Ecuador.

Ketty Vera ${ }^{2}$, Ketty Acuña ${ }^{1}$, Eulogio Vera ${ }^{3}$, Edith Aspiazu ${ }^{1}$, Rebeca Infante $^{I}$, Julio Solorzano

${ }^{1}$ Pediatría, Hospital Matilde Hidalgo de Procel, Ecuador

${ }^{2}$ Medicina General, Ministerio de Salud Pública, Ecuador

${ }^{3}$ Salud Pública, Vigilancia Epidemiológica, Ministerio de Salud Pública, Ecuador 


\section{Background}

The leading global epidemic Human Immunodeficiency Virus (VIH) infection has been well-documented ${ }^{1}$. It is transmitted from an infected person to an uninfected one by two ways: horizontal and vertical transmission (VT), which is mother-to-child transmission (MTCT) and is acquired at one or more of the following stages: transplacentally in the uterus during pregnancy, perinatally during the process of labor and delivery and postnanatally during breastfeeding ${ }^{1,2}$.

Objective

To demonstrate that adecquate management at each of these three moments reduces the $\mathrm{MTCT}^{2}$.

Methods

A observational-retrospective study was carried out at Maternidad Matilde Hidalgo de Procel in Guayaquil, Ecuador to detect the prevalence of serorevertors newborns of VIH who received prophylactic antiretroviral treatment at birth, formula milk and whose mothers got administered antiretroviral therapy (ART) during pregnancy or partum according to the established schemes $3^{, 4}$. These vertically exposed infants were followed up by an accredited pediatrician by the National Program of HIV-AIDS to receive special care during at least the first 18 months.

\section{Results}

One hundred (100) pregnant women were enrolled. ART was started between the 14th and 28th pregnancy week in a $41 \%$, after the 28th weeek in $24 \%$ and during labor or delivery in $35 \%$. One hundred percent of pregnant women received ART intrapartum. One hundred percent of the newborns received antirretroviral prophylaxis from 6 to 8 hours old for 4-6 weeks according to the applied scheme. In both, mothers and children, the most frequently administered regimen was the $\mathrm{C}$ with $48 \%$ based on zidovudine. One hundred percent of the newborns was fed by formula milk and $100 \%$ was serorevertor of HIV.

\section{Conclusions}

This study shows that MTCT was $0 \%$ due to the seroreversion in children at $=18$ months which represents that the treatments and properly applied procedures reduce the MTCT to zero and place Ecuador at the level of developed countries where the VT has been decreased at 1-2 \% ${ }^{5,6}$.

107

\section{Neonatology}

An Audit of Thyroid Function Testing Among Infants with Down Syndrome Over a Four Year Period

Catherine Breen ${ }^{l}$, Michael Boyle ${ }^{l}$

${ }^{1}$ Department of Neonatology, Rotunda Maternity Hospital, Ireland

\section{Introduction}

Infants with Down Syndrome (DS) have an increased risk of thyroid dysfunction and congenital hypothyroidism.[i] [ii] [iii]. Most countries have adapted TSH based screening for Congenital Hypothyroidism using a heel prick test[iv],[v] Irish and International guidelines recommend screening for thyroid dysfunction in Down Syndrome with the Newborn Screening Programme and repeating serum TSH and T4 at 6 months and annually thereafter.[vi]

Objectives

1. To determine the percentage of infants with Down Syndrome who undergo inappropriate thyroid function tests in the first three months of life. 2. To implement a pre-discharge checklist for neonates with DS.

\section{Methodology}

All neonates born with Down Syndrome between 1/1/2012 and 1/9/2016 were included. Blood results were reviewed retrospectively on the Hospitals APEX software system. If thyroid function testing was preformed before 3 months of age, charts were reviewed for an indication. The standard was based on the 'National Guideline for the Medical Management of Children and Adolescents in Ireland with Down Syndrome'. This states that Newborn Screening is an adequate screen for thyroid dysfunction in DS and venous thyroid function testing be carried out at least once every two years from their first year. A standard of $90 \%$ was set.

\section{Results}

One hundred babies with Down Syndrome were included. $40 \%(n=40)$ had serum thyroid function tests. $4 \%$ had a maternal history of thyroid dysfunction and $4 \%$ were repeated as requested by the National Newborn Screening programme. Thirty-two percent had no indication. Six of the 32 had abnormal repeat TSH but all had normal T4 levels.

\section{Conclusion}

Thirty-two percent had inappropriate thyroid function tests. This means unnecessary cost to the hospital, a poor use of time and staff resources taking the blood and an unnecessary physical and emotional burden to the patient and their parents. This was below the standard set. A checklist for all children diagnosed with Down Syndrome has now been introduced and this audit will be repeat in one year time.

138

Neonatology

Prediction of Hyperbilirubinemia in Term Infants Pi-Feng Chang ${ }^{I}$, Yu-Cheng Lin ${ }^{I}$, Kevin Liu ${ }^{I}$

${ }^{T}$ Pediatrics, Far Eastern Memorial Hospital, Taiwan

\section{Background and aims}

Recognition of neonates with high risk for significant hyperbilirubinemia is important. The aim of this study was to investigate the risk factors for significant hyperbilirubinemia in term infants.

\section{Methods}

A prospective cohort study was conducted to investigate the effects of birth weight, gestational age, sex, mode of delivery and feeding, glucose-6phosphate dehydrogenase deficiency, variant UDP-glucuronosyltransferase 1A1 (UGT1A1) gene, and solute carrier organic anion transporter 1B1 (SLCO1B1) gene on hyperbilirubinemia. The PCR- restriction fragment length polymorphism (RFLP) method was applied to detect the known variant sites in the UGT1Aland SLCOIB1 gene. Significant hyperbilirubinemia was defined as a bilirubin level exceeding the hour-specific phototherapy threshold. We analyzed the risk factors for significant hyperbilirubinemia using univariate logistic regression models.

\section{Results}

Totally 293 term infants (144 males and 149 females) were enrolled in this study. Thirty one (22 males and 9 females) had significant hyperbilirubinemia. The statistically significant risk factors for jaundice were G6PD deficiency $(37.38 ; 95 \% \mathrm{CI}, 4.58$ to $305.22 ; P=0.001)$, the $211 \mathrm{G}$ to $\mathrm{A}$ variation in the UGT1Al gene $(3.09 ; 95 \% \mathrm{CI}, 1.23$ to $7.74 ; P=0.016)$, vaginal delivery $(3.36 ; 95 \% \mathrm{CI}, 1.12$ to $10.05 ; P=0.03)$, breast feeding (4.91; $95 \%$ CI, 1.64 to $14.70 ; P=0.004)$, male $(2.89 ; 95 \%$ CI, 1.104 to $7.55 ; P=0.031)$ and gestational age $(0.46 ; 95 \% \mathrm{CI}, 0.29$ to $0.73 ; P=0.001)$. Conclusion

The infants who are G6PD deficiency, carry the 211 variants in the UGT1A1, vaginal delivery, low gestational age, male and breast feeding are at high risk to develop severe hyperbilirubinemia.

329

Neonatology

Bilistick ${ }^{\circledR}$ System as a reliable, cost-effective tool for predicting the need of treatment for hyperbilirubinemia in Low Middle Income Countries Carlos D. Coda Zabetta ${ }^{1}$, Chiara Greco ${ }^{1}$, Rinawati Rohsiswatmo ${ }^{2}$, Luciano Moccia ${ }^{3}$, Iman F. Iskander ${ }^{4}$, Stephen Oguche ${ }^{5}$, Chinyere Ezeaka ${ }^{6}$, Claudio Tiribelli ${ }^{1}$

${ }^{1} R \& D$ Department, Bilimetrix s.r.l., Italy

${ }^{2}$ Neonatology Division, Child Health Department, Cipto Mangunkusumo Hospital - University of Indonesia, Indonesia

${ }^{3}$ Research Department, Firetree Asia Foundation, China

${ }^{4}$ Department of Paediatrics, Cairo University Children Hospital, Egypt

${ }^{5}$ Department of Paediatrics, Faculty of Medical Sciences, University of Jos, Jos university Teaching Hospital, Nigeria

${ }^{6}$ Department of Paediatrics, Lagos University Teaching Hospital, Nigeria 


\section{Background}

In many Low Middle Income Countries, the access to laboratory support to estimate promptly bilirubin levels in jaundiced newborns is very difficult. This results in a delayed assessment of potentially dangerous jaundice increasing the risk of kernicterus. Implementation of inexpensive and simple devices to evaluate hyperbilirubinemia and treatment needs are essential to prevent neurological damages. We performed a multicenter study in 21 hospitals serving ethnically different populations distributed in Indonesia (7), Vietnam (9), Nigeria (4) and Egypt (1) to evaluate the assessment of the low cost Point-ofCare bilirubin assay Bilistick ${ }^{\circledR}$ System (BS) in determining hyperbilirubinemia and treatment needs according to NICE guidelines.

\section{Objective}

To determine the effectiveness of BS to assess neonatal hyperbilirubinemia and indicate the appropriate treatment according to NICE guidelines.

\section{Methods}

Blood samples were obtained in 1016 healthy $\geq 36$ weeks of gestation newborns, presenting visual symptoms of neonatal jaundice. Bilirubin level was assessed by laboratory and BS methods; age of the patient was recorded at admission. The accuracy of BS in predicting the need for phototherapy or exchange transfusion treatment was evaluated according to NICE Guidelines for neonatal jaundice management. The bilirubin level obtained by laboratory assay was used as control for treatment prediction.

\section{Results}

The prediction of BS to determine need of phototherapy (Table 1A) was accurate in $88 \%$ of the cases, with a sensitivity of $77 \%$, specificity of $94 \%$, Positive Predictive Value (PPV) of $87 \%$ and Negative Predictive Value (NPV) of $88 \%$. BS was accurate in predicting the need for exchange transfusion therapy (Table 1B) in $99 \%$ of the cases, with a sensitivity of $90 \%$, specificity of $99 \%$, PPV of $93 \%$ and NPV of $99 \%$.

Table 1A - Prediction of Phototherapy Treatment using Bilistick ${ }^{\oplus}$ System

\begin{tabular}{|c|c|c|c|c|c|c|c|c|}
\hline & \multicolumn{5}{|c|}{$\begin{array}{l}\text { NICE Guidelines recommendation } \\
\text { according to Lab bilirubin result } \\
\text { (Control) }\left(^{*}\right)\end{array}$} & \multirow{2}{*}{\multicolumn{2}{|c|}{ Prediction analysis by BS }} \\
\hline & & A & B & C & D & $\mathbf{E}$ & & \\
\hline \multirow{5}{*}{$\begin{array}{l}\text { NICE Guidelines } \\
\text { recommendation } \\
\text { according to BS } \\
\left.\text { bilirubin result ( }{ }^{*}\right)\end{array}$} & A & 510 & 34 & 40 & 2 & 0 & True negative & 619 \\
\hline & B & 17 & 58 & 36 & 3 & $o$ & False negative & 81 \\
\hline & $\mathrm{c}$ & 18 & 14 & 99 & 56 & o & True positive & 275 \\
\hline & D & 6 & 3 & 4 & 84 & 3 & False positive & 41 \\
\hline & E & 0 & 0 & 0 & 2 & 27 & TOTAL & 1016 \\
\hline
\end{tabular}

Table 1B - Prediction of Exchange Transfusion using Bilistick ${ }^{\oplus}$ System

\begin{tabular}{|c|c|c|c|c|c|c|c|c|}
\hline & \multicolumn{5}{|c|}{$\begin{array}{l}\text { NICE Guidelines recommendation } \\
\text { according to Lab bilirubin result } \\
\text { (Control) (*) }\end{array}$} & \multirow{2}{*}{\multicolumn{2}{|c|}{ Prediction analysis by BS }} \\
\hline & & A & B & c & D & $\mathbf{E}$ & & \\
\hline \multirow{5}{*}{$\begin{array}{l}\text { NICE Guidelines } \\
\text { recommendation } \\
\text { according to BS } \\
\text { bilirubin result (*) }\end{array}$} & A & 510 & 34 & 40 & 2 & $o$ & True negative & 984 \\
\hline & B & 17 & 58 & 36 & 3 & $o$ & False negative & 3 \\
\hline & C & 18 & 14 & 99 & 56 & 0 & True positive & 27 \\
\hline & D & 6 & 3 & 4 & 84 & 3 & False positive & 2 \\
\hline & E & 0 & 0 & 0 & 2 & 27 & & 1016 \\
\hline
\end{tabular}

(*) NICE Guidelines recommendation

A - Negative

B - Repeat bilirubin measurement in 6-12 hours

C-Consider phototherapy and repeat bilirubin

measurement in 6 hours

D-Start phototherapy

- Perform an exchange transfusion unless the bilirubin level falls below threshold while the treatment is being prepared

\section{Conclusion}

Bilistick ${ }^{\circledR}$ System is a cost-effective POC bilirubin assay able to accurately predict treatment in the case of neonatal hyperbilirubinemia according to NICE Guidelines recommendations.

473

Neonatology

Transport Characteristics of Referred Newborns and Their Outcomes: Indian Perspective

Poonam Dalal $^{1}$, Jasbir Singh ${ }^{1}$, Geeta Gathwala ${ }^{I}$

${ }^{T}$ Pediatrics, Pt. B.D. Sharma PGIMS Rohtak, Haryana India, India

\section{BACKGROUND}

Safe and specialized neonatal transport is a significant determinant for favourable outcome among sick neonates, yet it is often inaccessible and unorganized in developing countries like India. The National Family Health Survey (NFHS-4) shows encouraging improvements in infant and under-five mortality rates in India over last few years, however, the single digit neonatal mortality rate still remains a distant dream.

\section{OBJECTIVES}

To determine the transport characteristics and define predictors of mortality among newborn babies referred to a tertiary care centre of North India.

\section{METHODS}

This descriptive study was conducted from 15th Jan 2016 to 30th April 2016 and included babies who were referred to a tertiary care centre of North India. Baseline characteristics of referred newborns, transport practices observed, clinical presentation and condition of babies at arrival were recorded. The data was evaluated to define predictors of mortality among the transported newborns.

\section{RESULTS}

A total of 301 newborns were enrolled during the study period. The most common indication for transport was prematurity $(40 \%)$ followed by need for mechanical ventilation ( $38 \%$ ) and birth asphyxia (28\%). Most neonates were transported by government ambulance services $(84 \%)$ followed by private ambulances $(12.5 \%)$ and private vehicles $(3.5 \%)$. Paramedic personnel accompanied the babies in $85 \%$ of cases. About $60 \%$ babies got referred within 12 hours of life, $73 \%$ in 24 hours and $85 \%$ presented within first week. Ninety babies out of 301 died. Out of these $45 \%$ expired within 12 hour of presentation. Hypothermia at time of admission (P0.0001), shock at presentation (P0.0001) and extreme low birth-weight (P0.0001) were found to be the most significant predictors of mortality among the transported newborns. CONCLUSION

The presently observed neonatal transport practices in India are suboptimal. Pre-transport stabilization, dedicated teams for neonatal transport and prevention of hypothermia before and during transport seem to be promising interventions to improve neonatal outcomes.

\section{8}

Neonatology

Pathogen Specific Changes in Core Temperature and NeuroInflammation in The Neonatal Rat

Mari Falck ${ }^{1}$, Damjan Osredkar ${ }^{1,2}$, Elke Maes ${ }^{1}$, Torun Flatebo ${ }^{I}$, Thomas Wood $^{I}$, Hemmen Sabir ${ }^{1,3}$, Marianne Thoresen ${ }^{1,4}$

${ }^{1}$ Department of Physiology, University of Oslo, Institute of Basic Medical Sciences, Norway

${ }^{2}$ Department of Paediatric Neurology, University Children's Hospital, Slovenia ${ }^{3}$ Department of General Paediatrics, Neonatology and Paediatric Cardiology, University Children's Hospital, Heinrich-Heine University, Germany

${ }^{4}$ School of Clinical Sciences, University of Bristol, UK

\section{Background}

Perinatal infections increase vulnerability of the neonatal brain to hypoxic-ischemic (HI) injury. We recently demonstrated 
experimentally how inflammatory pre-sensitisation through TLR-4 (LPS) abolished the effect of therapeutic hypothermia (TH) after HI. Remarkably, when inflammation is induced through TLR-2 $\left(\mathrm{PAM}^{3} \mathrm{CSK}^{4}\right)$, TH provides $80 \%$ neuroprotection ${ }^{1,2}$. A sepsislike condition is induced in both situations, but how they differ with respect to neuro-inflammatory responses in the newborn is unknown.

\section{Objective}

To examine physiological and neuro-immunological changes induced in the neonatal rat by triggering inflammation systemically using LPS or $\mathrm{PAM}^{3} \mathrm{CSK}^{4}$ (PAM), compared to saline.

\section{Methods}

After a systemic injection of $0.9 \% \mathrm{NaCl}$ (Veh), LPS, $(0.1 \mathrm{mg} / \mathrm{kg}$ ) or PAM $(1 \mathrm{mg} / \mathrm{kg})$ in P7 rats, core temperature was measured 2-hourly for $24 \mathrm{~h}$. Brain tissue was analyzed for pro-inflammatory cytokines (IL-6, IL-1 $\beta$, TNF- $\alpha$, and IL-10) (PCR) and apoptosis (cCas3) and microglial activation (Iba1) (WB).

\section{Results}

LPS induced a significant drop in core temperature after injection compared to Veh $(p=0.01)$ and PAM $(p=0.01)$ (Fig 1). Temporal expression of IL-6, IL-1 $\beta$, TNF $\alpha$ and IL-10 differed significantly after injection of LPS and PAM, with expression peaks after different incubation lengths (Fig 2, A: PAM, B: Veh, C: LPS). cCas 3 was significantly increased 24-48 hours after LPS injection compared to PAM and Veh $(p=0.0002)$ which were similar. Iba1 was increased by systemic LPS and PAM compared to $\operatorname{Veh}(p=0.07)$.

\section{Conclusion}

Experimental induction of a sepsis-like condition through TLR-2 and TLR-4 induced neuro-inflammation. The gram-positive pathway (PAM) creates a neuro-inflammatory state very different from that of the gram-negative pathway (LPS). The difference in core temperature preservation indicates a role of metabolic demands during infection that might be pathogen specific. These results highlight the need for more research on inflammatory cross-talk between blood and brain, and the importance of pre-clinical models being carefully tailored to their clinical scenario.

1. Osredkar, D. et al. Resuscitation 85, 567-572 (2014).

2. Falck, M. et al. http://www.abstracts2view.com/pas/ view.php?nu=PAS16L1_4615.4 (2016).

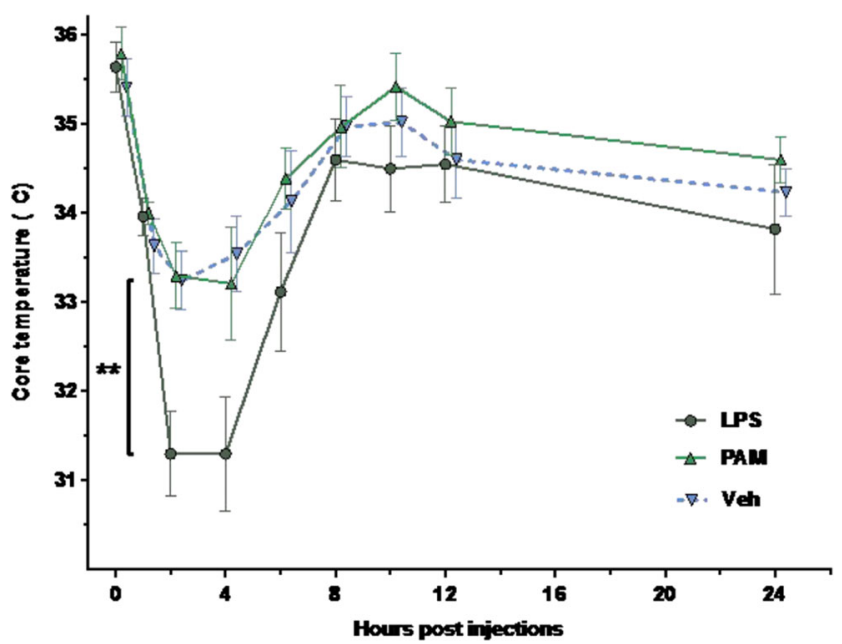

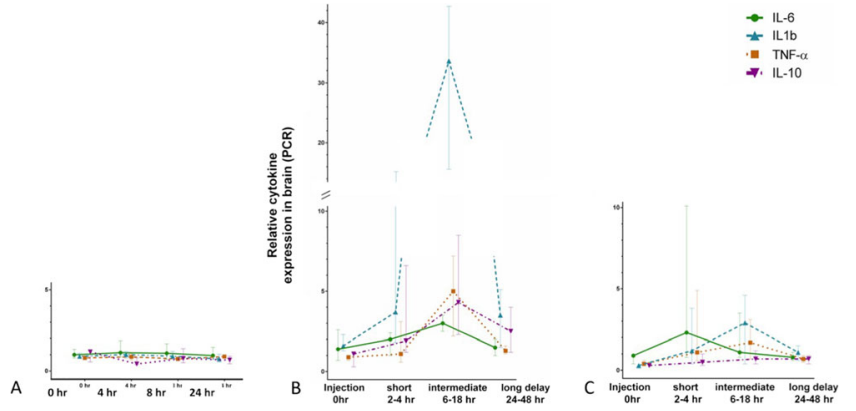

802

Neonatology

Enterovirus Meningitis in Neonates

${ }_{\text {Petja Fister }}{ }^{1}$, Dominika Žugelj ${ }^{1}$, Mojca Kavčič ${ }^{1}$, Liza Lah ${ }^{2}$, Darja ParoPanjan ${ }^{I}$

${ }^{1}$ Division of Paediatrics, Department of Neonatology, University Medical Centre Ljubljana, Slovenia

${ }^{2}$ Department of Infectious Diseases, University Medical Centre Ljubljana, Slovenia

\section{Background}

Enterovirus infection is common in children, but less prevalent in neonates. It causes nonspecific febrile disease, aseptic meningitis, hepatitis with coagulopathy and/or myocarditis.

\section{Objective}

To evaluate the burden and clinical characteristics of enterovirus meningitis in a tertiary neonatal unit from 2006 to 2016.

\section{Methods}

We included neonates with positive lumbar puncture $(21 \pm 10$ leukocytes $/ \mathrm{mm}^{3}$ ) and enterovirus RNA detected in the cerebrospinal fluid (CSF). We analysed maternal and perinatal history, clinical and laboratory data, therapy and short-term outcomes.

\section{Results}

A total of 21 neonates were included, all were term, all but one had appropriate birth measures, 15 were male $(71 \%)$. Most patients presented during the summer and autumn months. We found two peaks of disease onset: 5 to 9 and 18 to 29 days (median values 7 and 21). Eight patients were in contact with siblings, who had signs of viral disease. Three mothers were ill at delivery or shortly after. Twenty percent neonates presented with irritability, 16 with fever (median $38.6{ }^{\circ} \mathrm{C}$ (37.5-39.5)), 16 with feeding intolerance, 15 with diarrhoea, 6 with nonspecific rash, 1 had seizures. Seven neonates had mildly elevated CRP (range 9-31 mg/L) and 7 had elevated PCT (range 0.53-10 ug/L). All had elevated leukocyte count in the CSF (median $11 / \mathrm{mm}^{3}$ (3-1739)). None had clinical or laboratory signs of SIRS, hepatitis with coagulopathy and/or myocarditis. In 15 neonates antibiotics were started, median time of treatment was 1 day. All patients had good outcome.

\section{Conclusions}

Enterovirus infection is an important cause of meningitis in neonates during summer and autumn months, particularly with positive history of contact with ill persons, with persistent fever, irritability and normal or mildly elevated CRP and/or PCT values. Males have a higher risk of disease. Our findings suggest that enterovirus meningitis in neonates may be transmitted in two ways and carries a good prognosis. 
732

\section{Neonatology}

An Epidemiological Study of Retinopathy of Prematurity in the Maltese Population

Matthew Fenech ${ }^{I}$, Paul Soler ${ }^{I}$

${ }^{1}$ Paediatrics and Neonatology, Mater Dei Hospital, Malta

\section{Background}

Retinopathy of Prematurity (ROP) is a proliferative retinal disease, characterized by abnormal development of retinal vessels in children of young gestational age and low birth weight. It remains a leading cause of childhood blindness, with more than 30,000 children affected worldwide annually.

\section{Objective}

Our primary objective is to assess the mean gestational age and birth weight for the development of ROP within the Maltese population. Our secondary objectives are to identify any correlation between pre-natal steroid us and maternal diabetes with the development of ROP.

\section{Methods}

A retrospective review of the ROP register from the Paediatric and Neonatology Department, Mater Dei Hospital Malta was undertaken. All patients screened for ROP between January 2012 to April 2017 were included. The notes of each infant as well as the maternal notes were reviewed in order to collect and collate the data. Cases with incomplete or unavailable data were not used for the purposes of this study.

\section{Results}

The data has not yet been analyzed due to the time restrictions for submission of this abstract. That being said, the results will prove to be interesting considering the high prevalence of diabetes amongst the Maltese population coupled with the common current practice of antenatal steroid use in pre-term infants. Nonetheless, the small genetic pool of the Maltese population will shed light onto the influence genetics may have on the course of ROP should any discrepancy in the results of the primary objectives be obtained compared to already published data.

\section{Conclusion}

We hypothesize that the mean birth weight and mean gestational age in infants with ROP on the Maltese islands could vary from already published data, with some influence coming from the large prevalence of diabetes mellitus amongst mothers of childbearing age.

Key Words: ROP, Malta, Epidemiology, Diabetes mellitus, pre-natal steroids

\section{0}

\section{Neonatology}

Myocarditis from fetal to neonates: Our experience to diagnosis treatment and outcomes in a single centre.

Olga Filaretova ${ }^{3}$, Anna Kotova ${ }^{1}$, Margarita Tumanyan ${ }^{1,3}$, Elena Bespalova ${ }^{2}$ ${ }^{T}$ Intensive Cardiology for neonates\&infants with congenital heart disease, Bakoulev Scientific Center for Cardiovascular Surgery, Russia

${ }^{2}$ Perinatal Cardiological Center, Bakoulev Scientific Center for Cardiovascular Surgery, Russia

${ }^{3}$ Pediatric Cardiology Course at the Chair of Cardiovascular Surgery, Moscow State University of Medicine and Dentistry, Russia

\section{Aim}

We performed a retrospective analysis of 89 fetal echograms (in between 01.2015 and 10.2016).

\section{Methods}

Prenatal ultrasound screening included 2-D and 3/4-D EchoCG. Diagnosis was confirmed either by postnatal EchoCG or autopsy; the percentage of prenatal mistakes was $0 \%$. After birth newborns underwent routine cardiology examination with EchoCG, diagnostic blood tests, virus antibody tests.

\section{Results}

$89 \backslash 2586$ (3.4 \%) CHD foetuses with a prenatal diagnosis of myocarditis were enrolled. Initial fetal EchoCGs were obtained between 12 and 39 weeks of gestation (median 24.5 weeks). Diagnostic ultrasound criteria and complications: dilatation of LV(87/89) and dilatation of $\mathrm{RV}(2 / 89)$ with poor ventricular function; $\mathrm{AV}$ regurgitation and pericardial effusion(89/89); fetal non-immune hydrops(21/89); fetal arrhythmias AVB, bradyarrhythmia(11/89). Outcomes for the cases with fetal myocarditis: the termination of pregnancy $9 / 89(10.1 \%)$; intrauterine fetal death 4/89(4.5\%), neonatal death soon after birth 6/89(6.7\%), fetal successful treatment 67/89(75.2\%). Of 67 newborns $25(37 \%)$ were a previously successful cases of fetal myocarditis treatment; also $25(37 \%)$ cases had CHD, of which 16/25(64\%) were critical CHD (CCHD). In all $\mathrm{CHD}$ cases myocardial antibody titre was higher than the reference value. Of virus antibody tests positive results were obtained for: CMV (20/25), HHV type 1(165), and EBV (46). Patients with CHD and myocarditis combined were indicative of heart failure (mean NYHA class 3.1): pericardial effusion (10/25), dilatation heart cavities or myocardial hypertrophy (195) with reduction systolic function. The CHD in newborns with prenatal myocardities were primarily represented by critical CHD 165 (64\%). All patients underwent the following active treatment: glucocorticosteroids; NSA; anti-virus/anti-bacterial; beta blocker, heart failure and arrhythmia therapy. Neonates with CCHD required emergent surgical intervention due to CCHD incidence and other planned CHD repair. Before 1 year 3(3.3 \%) infants with AVB underwent pacemaker implantations. Hospital mortality was $0 \%$.

\section{7}

\section{Neonatology}

Cool babies, not so cool....

Rebecca Finnegan ${ }^{1}$, Siobhan Hackett ${ }^{1}$, Ireti Farombi ${ }^{l}$

${ }^{T}$ Department of Neonatology, Our Lady of Lourdes Hospital, Drogheda, Co Louth, Ireland

\section{Aims}

- To assess the incidence of hypothermia in newborns in our institution

- To develop a care bundle to reduce the incidence of hypothermia Methods

This is a prospective audit of babies born over an eight week period (October-December 2016). A Plan-Do-Study-Act cycle was used. Prior to commencement, staff were educated on the mechanisms of reducing heat loss in the newborn. A quality improvement care bundle was developed and used to collect data. Data was reviewed and analysed on a weekly basis and improvement steps were implemented. Babies born in the labour ward during a one-week period of the study were used as control.

\section{Results}

There were 200 babies born during the study period; 166 (83\%) of these were included in the study (10\% were preterm). Eighteen infants in the study had hypothermia on admission to the postnatal ward. In the hypothermia group, a third of these were preterm; median gestational age was 36 weeks. The median birth weight was $2.79 \mathrm{~kg}$ (range $1.26 \mathrm{~kg}-4.12 \mathrm{~kg}$ ). The average room temperature recorded was lower in one theatre being $22.5^{\circ}$ compared to another theatre $\left(24.9^{\circ}\right)$. Increasing the temperature in theatre to the optimal level of $23-25^{\circ}$ reduced the incidence of hypothermia. Three babies (17\%) were hypothermic prior to leaving theatre. The average room temperature recorded in the labour ward was $24.8^{\circ}$. Only two babies born on the labour ward during the one week audit period had hypothermia on admission to the postnatal ward.

\section{Conclusions}

Eleven percent of babies born in the lower temperature environment had hypothermia on admission to postnatal ward, compared to $3 \%$ of babies born in the higher temperature environment

Recommendations:

- Continued education of staff and implementation of care bundle

- Ensuring delivery environmental temperature is set between 23$25^{\circ}$ degrees

- Transport incubator to be used when transferring preterm infants/low birth weight infants from delivery to NICU 
303

\section{Neonatology}

Incidence and risk factor for the development of hearing impairment in infants treated with Therapeutic Hypothermia for Hypoxic Ischaemic Encephalopathy

Michael Fitzgerald $^{1}$, Breda Hayes ${ }^{I}$, Adam Reynolds ${ }^{I}$, Gary Norman ${ }^{2}$, Cliona Hughes $^{3}$

${ }^{I}$ Department of Neonatology, Rotunda Hospital, Ireland

${ }^{2}$ Department of Audiology, Rotunda Hospital, Ireland

${ }^{3}$ Department of Statistics, Children University Hospital Temple Street,

Ireland

Therapeutic hypothermia for moderate to severe hypoxic ischaemic encephalopathy (HIE) has been associated with an increased risk of longterm hearing loss ${ }^{1}$ Our objective was to establish the local incidence of hearing loss in newborns with HIE and to identify risk factors associated with hearing loss.

A retrospective case note review was conducted between June 2012 and March 2016 in a tertiary neonatal intensive care unit in Dublin, Ireland. Eligibility criteria were HIE and enrolment in the National Newborn Hearing Screening Programme (AOAE and AABR).

Information collected included demographic data, labour and delivery details along with detailed neonatal history including resuscitation, ventilation, seizure and medication history. Measures of renal function, gentamycin levels and brain magnetic resonance imaging (MRI) findings were reviewed.

Fishers exact test and both Kruskall Wallis rank and Pearson's $r$ correlation coefficient were used to analyse continuous and categorical variables respectively.

Fifty-seven newborns received therapeutic hypothermia for HIE. Fifteen infants were excluded from the study (12 died and 3 had incomplete data). Complete data was available for 42 babies, 4 of whom had significant hearing impairment.

The development of hearing loss was associated with the use of nitric oxide $(p=0.046)$, diuretics $(p=0.002)$, inotropes $(p=0.032)$ and abnormal blood glucose levels $(p=0.006)$. Hearing loss was also associated with a low Apgar Score at 1 minute $(p=0.0219)$, high creatinine on days 1 and 2 of life $(p=0.0172$ and 0.0198$)$ and raised liver function tests (AST $p=0.0012$, ALT $p=0.0037$ ).

Hearing impairment is common in survivors of term HIE treated with hypothermia. Hypoglycaemia should be aggressively treated in this population.

Larger studies are required to fully identify risks associated with hearing impairment in this population.

Smit, E. et al. Factors associated with permanent hearing impairment in infants treated with therapeutic hypothermia. J. Pediatr. 163, 995-1000 (2013).

346

\section{Neonatology}

Interpretation of Umbilical Venous Catheter position on X-ray: a trainee's perspective.

Eleanor Katherine Harrison ${ }^{1}$, Kate Lydekker ${ }^{1}$, Mark Sellwood ${ }^{l}$

${ }^{T}$ Neonatal Intensive Care Unit, University College London Hospital, UK

\section{Background}

As paediatric trainees working within a tertiary neonatal unit, we observed uncertainty amongst fellow trainees when assessing umbilical venous catheter (UVC) position on X-rays. This had two potential adverse consequences; the unnecessary removal of safely positioned lines and the continued use of malpositioned UVCs.

\section{Objective}

To analyse the accuracy of trainees' interpretation of UVC placement on X-ray within our department.

Methods

Through 'BadgerNet Neonatal' we identified all patients undergoing UVC insertion within our department over a four-month period $(n=83$, images available in 78 cases). With a senior neonatal consultant, we retrospectively reviewed the accompanying X-ray images against British Association of Perinatal Medicine guidelines. Medical notes for 52 patients were available, allowing assessment of trainee interpretation of position at the time of insertion, and if any adjustments were made. Each image was categorised as either safe or unsafe for use.

\section{Results}

$\begin{array}{lll}\text { Assessment } & \text { Number of UVCs ( } \mathrm{n}=78) & \text { Number of UVCs (where notes available, } \mathrm{n}=52) \\ \text { Safe for use } & 61 & 38 \\ \text { Unsafe for use } & 17 & 14\end{array}$

Seventy-eight percent of UVCs inserted were deemed safe for use on retrospective assessment. Where assessable, $8 \%(n=3)$ of safely positioned UVCs were unnecessarily removed. Worryingly, $24 \%(n=4)$ of malpositioned UVCs were not adjusted. In two cases, these UVCs were subsequently found to lie within the liver parenchyma, resulting in TPN peritonitis in one instance. Conclusion

Our study highlights difficulties faced by paediatric trainees in the interpretation of UVC position on X-rays. Available guidelines focus upon a narrow range for the acceptable height of a line, with no commentary on the expected trajectory. This led to safe but not 'perfectly' positioned lines being removed as well as unsafe lines being left in position. We have therefore collated our X-ray images to create an interactive educational toolkit for trainees. We hope increased exposure to images will improve trainees' confidence in neonatal anatomy and X-ray interpretation, reducing adverse patient events.

\section{5}

Neonatology

The Impact of Placental Pathology on Necrotising Enterocolitis (NEC)

Abu Mohammed Ismail ${ }^{1}$

${ }^{T}$ NICU, Sickkids Hospital, Canada

\section{Background}

The placenta has recently become an organ of interest in the search for causes of preterm necrotising enterocolitis.Placental pathology are associated with preterm mortality and morbidity. However, the impact of placental pathology on NEC is inconsistent across studies. This study explores the role of placental pathology in predisposing to NEC pathogenesis.

\section{Objectives}

To determine the association between placental pathology with clinical and radiological diagnosis of NEC

\section{Methods}

One hundred eight neonates (mean gestational age 28.5 weeks and mean birth weight $1162 \mathrm{~g}$ ) were studied retrospectively over 10 years (2003 to 2013). Based on placental pathology they were classified as P1: maternal placental vascular pathology; P2: fetal placental vascular pathology; P3: placental inflammatory-immune pathology and clinical diagnosis and radiological of NEC (grade 2 Bells). SAS method was used for statistically analysis. 
Results

All 108 neonates had NEC (grade 2 Bells). P2 $N=58(53.7 \%)$, P1 $N=33(30.5 \%), \mathrm{P} 3 N=17(15.7 \%)$

Conclusion

Placental abnormalities, especially fetal vascular lesions put preterm neonates at risk of NEC. The results of this study explore the risk placental infection may contribute to pathogenesis of NEC and warrants further study

\section{7}

\section{Neonatology}

Translating Lessons Learnt from the Dead Babies into Action in Resource-limited Settings: Perinatal Death Audits in Sri Lanka Kapila Jayaratne $^{I}$

${ }^{T}$ Ministry of Health, Family Health Bureau, Sri Lanka

\section{Background}

With the reduction of infant mortality rate to a single digit ( 8.2 per 1000 live births) and a larger proportion (60\%) of such deaths being early neonatal deaths (END), perinatal deaths (PND) emerge as a priority area in further reducing neonatal mortality in Sri Lanka -a low and middle income country in South Asia.

National Perinatal Mortality Surveillance system was implemented in 2006. All specialized hospitals (with an obstetrician/paediatrician) are required to document all PNDs, review them monthly at a hospital stakeholder meeting and send a report to Ministry of Health. Structured data collection formats, monthly reporting formats and guidelines are available.

\section{Objective}

To analyze national PND data for meaningful outcomes

Methods

In 2014, data were received from all hospitals (both government and private sector) with labour rooms from a total of 452 hospitals ( 74 Specialized government hospitals, 357 Non-specialized peripheral hospitals and 21 Private hospitals). Considering total live births reported by Civil Registration System (349715), coverage of live births in this study was $99.6 \%(n=348362)$.

\section{Results}

The analysis included 1386 (46\%) stillbirths and 1623 (54\%) ENDs (Total 3009 PNDs). Stillbirth and early neonatal mortality rates were 4.0 per 1000 total births and 4.7 per 1000 live births respectively. The perinatal mortality rate was 8.6 per 1000 total births. Majority of PNDs $(52.1 \%)$ were male. One third $(35.7 \%)$ of PNDs occurred in primies. Birthweight $1000 \mathrm{~g}$ was reported in $20 \%$ of all PNDs. Nearly $10 \%$ of ENDs had a period of gestation.

\section{Outcome}

Lessons learnt were translated into action at hospital, district and nationallevels. Actions included; prevention strategies on premature deliveries, expansion of premature baby units, strategies on birth defects prevention, early neurodvelopmental care, neonatal retrieval systems and introducing therapeutic body cooling. With contribution from PND surveillance, Sri Lanka targets a neonatal mortality of 2.5 by year 2025 .

619

\section{Neonatology}

Barriers to implementing NICE Guidelines for early onset neonatal infection - Cross-sectional survey of neonatal blood culture reporting by UK laboratories

Siba Prosad Paul ${ }^{1}$, Edward Caplan ${ }^{2}$, Hannah Morgan ${ }^{3}$, Paul Christopher Turner ${ }^{4}$
${ }^{1}$ Paediatrics, Torbay Hospital, UK

${ }^{2}$ Medical School, University of Bristol, UK

${ }^{3}$ Medical School, Peninsula College of Medicine \& Dentistry, UK

${ }^{4}$ Medical Microbiology, Torbay Hospital, UK

\section{Background}

In 2012, the National Institute for Health and Care Excellence (NICE) published guidelines for managing early-onset neonatal infections. It recommended starting antibiotics early for neonates with suspected infection and to discontinue them at 36 hours if blood culture were negative, initial clinical suspicion wasn't strong, clinical condition was reassuring and CRP was satisfactory.

\section{Aims}

To explore how neonatal blood cultures are reported by microbiology laboratories in the UK, to ascertain current practice and to facilitate better implementation of NICE guidelines.

\section{Methods}

Cross-sectional survey was conducted amongst biomedical scientists via telephone interview involving all 208 UK laboratories attached to hospitals providing neonatal services. Respondents were asked about awareness of NICE guidelines, if paediatric blood culture bottle were used, if tests were performed 'on-site' and whether there was a system for publishing results automatically out-of-hours including how positive/negative results were communicated.

\section{Results}

198/208 (95.2\%) of laboratories responded. 125 (63.1\%) laboratories did blood culture analysis 'on-site'. 6/125 biomedical scientists were not aware of the NICE guidelines and 5/125 centres used adult blood culture bottles. Communication to clinicians for negative cultures by 36 -hours was available out-of-hours using an automated reporting system in 56/125 (44.8\%). For positive blood culture a similar reporting system was available in $32 / 125$ $(25.6 \%)$ laboratories

\section{Conclusions}

Fifty-five percent laboratories were not reporting negative blood culture results out-of-hours while $74 \%$ laboratories depended on manual systems for positive results. Standardisation of blood culture reporting at 36 hours is essential for proper implementation of the NICE guidelines and better service delivery including improved antimicrobial stewardship.

\section{1}

\section{Neonatology}

Development of Children Born to Female Liver or Kidney Recipients Bozena Kociszewska-Najman ${ }^{l}$, Joanna Schreiber-Zamora ${ }^{l}$, Bronislawa Pietrzak ${ }^{2}$, Natalia Mazanowska ${ }^{2}$, Miroslaw Wielgos ${ }^{2}$

${ }^{1}$ Division of Neonatology and Neonatal Intensive Care Unit of the First Department of Obstetrics and Gynaecology, Medical University of Warsaw, Poland

${ }^{2}$ First Department of Obstetrics and Gynecology, Medical University of Warsaw, Poland

\section{BACKGROUND}

Neonates born to transplant recipients have higher rate of prematurity and low-birth-weight. Chronic immunosuppression during pregnancy may have adverse effect on developing fetus and impair later development of the neonate, infant and child.

\section{OBJECTIVE}

The aim of this study was to assess the neurological and somatic development of children born to mothers after liver (LT) or 
kidney (KT) transplantation exposed to chronic immunosuppression in utero.

\section{METHODS}

Study group involved 82 children, including 46 children in LT and 36 children in KT group. Control group consisted of 82 children born to healthy mothers. Neurologic abnormalities were divided into light, moderate and severe. In the somatic assessment body weight, body mass index (BMI), length/height and head circumference of children were included. Anthropometric measurements and BMI were compared to the percentile values matched to the sex and age.

\section{RESULTS}

Neurological examination was normal in 40 (86.9 \%) LT children and in $31(86.1 \%) \mathrm{KT}$ children. Light disorders were found in $5(10.9 \%)$ LT children and in $4(11.1 \%)$ KT children. Moderate disorders were present in $1(2.2 \%)$ LT child and in 1 $(2.8 \%)$ KT child. There were no severe disorders (cerebral palsy). Percentage of abnormalities in neurological examination was similar in study and control groups $(p=0.32)$. In the somatic assessment higher prevalence of obesity was found in study group (16.4\%) compared to controls $(6.3 \%), p=0.037$. Obesity was observed in both groups, more frequently (LT $17.1 \%$ and KT $15.4 \%)$ than in general population $(5 \%)$ (p0.05). This difference was not observed in control groups. Tacrolimus prenatal exposure seems to associate with increased risk of obesity in children.

\section{CONCLUSIONS}

Chronic immunosuppressive treatment does not affect negatively neurologic development of the children born to graft recipients. Somatic differences, in particular obesity in children born to posttransplant mothers, may be associated with immunosuppressive treatment used in pregnancy.

\section{2}

\section{Neonatology}

\section{Therapeutic Hypothermia in Latvia}

Sabine Laktina $^{1,2}$, Aleksandra Jurasa ${ }^{3}$, Sandija Stanke ${ }^{3}$, Daiga Kviluna ${ }^{3}$ ${ }^{T}$ Science and Education, University Children's Hospital, Latvia

${ }^{2}$ Residency, Riga Stradins University, Latvia

${ }^{3}$ Nneonatology, University Children's Hospital, Latvia

\section{Background}

Therapeutic hypothermia ( $\mathrm{TH})$ has now become a standard of care for term and near term infants with moderate to severe hypoxicischemic encephalopathy. The method is protective by inhibiting various pathophysiological events in the cascade of injury. It has recently been proven to be the only medical intervention which reduces brain damage and improves an infant's chance of survival and reduced disability. Neonatal TH in Latvia was started in 2012 - in University Children's Hospital using whole body hypothermia (Criticool) and Riga Maternity Hospital presenting selective head cooling (Cool Cap). Since 2016 whole body hypothermia (Thecoterm) is also available in Pauls Stradins Clinical University Hospital.

\section{Objective}

Provide the information about our experience and results using TH in Latvia during 2012-2016 in newborns with moderate and severe hypoxic- ischemic brain damage.

\section{Methods}

In retrospective research, a total of 107 newborns with moderate and severe HIE receiving therapeutic hypothermia in tree hospitals were included in our analysis.

\section{Results}

Mortality rate $-17.76 \%(19 / 107)$ is comparable with other European studies. At age 1 year 48 patients underwent neurodevelopmental examination. $33(68.75 \%)$ - have normal neurological development; $7(14.58 \%)$ - spastic tetraparesis and seizures; $4(8.33 \%)$ - hemiparesis; $3(6.25 \%)$ - mild motor development retardation; $1(2.08 \%)$ - isolate language delay Conclusions

$69 \%$ of the children whose neurological development was examined at the age of 1 had an age-appropriate psychomotor development. For $40 \%$ of the treated patients an MRI scan showed no signs of hypoxic ischemic damage - a finding that has a high correlation with good psychomotor development. There is no data in Latvia on the neurological development of patients of hypoxic ischemic encephalopathy who have not received $\mathrm{TH}$, since there is no unified dynamic follow-up of these children.

\section{7}

Neonatology

The Rat 50/10 Oxygen-induced Retinopathy As a Potential Model of Bronchopulmonary Dysplasia

Huijuan Li ${ }^{1}$, Ruyuan $\mathrm{Zhu}^{1}$, Li Jiang ${ }^{1}$

${ }^{T}$ Pediatrics, Zhongda Hospital Affiliated to Southeast University, China

\section{Background}

Bronchopulmonary dysplasia (BPD) is a chronic lung disease that affects preterm babies, characterized by abnormally greater amount of myofibroblast and alveolar walls overwhelmed by collagen. Rodent models of BPD are dominated by hyperoxia insults, while these models are in urgent need of modification due to that pure hyperoxia is not consistent with the clinical status in most of the NICUs nowadays. The rat 50/ 10 oxygen-induced retinopathy (OIR) model is used in the study of retinopathy of prematurity (ROP) and becoming widely accepted these years resulting from its consistency with clinic.

\section{Objective}

In pursuit of a better model of BPD, we conducted OIR and analyzed the pathological changes of lungs.

\section{Methods}

The rat OIR model was conducted by putting the newborn rat into cycled $50 \%$ and $10 \%$ oxygen (from the day of birth, each concentration for 24 hours), lasting for 14 days and then exposed to normoxia for another 4 days. Lungs were harvested at both day 14 (D14) and D18 for HE, $\alpha$-SMA and Picrosirius Red staining.

\section{Results}

At D14, lungs in the OIR group presented with fewer and enlarged alveolar. At D18, the alveolar enlarged further and the septa thickened. In $\alpha$-SMA, it was higher in OIR groups, indicating more myofibroblast localization. At D18, when myofibroblast was almost absent from the alveolar walls in control group, the OIR lungs had persistence expression of $\alpha$-SMA. In Picrosirius red, the amount of collagen in OIR group was higher than that of control group at both time points and the trend was more obvious at D18.

\section{Conclusion}

The prolonged and stronger existence of myofibroblast produced and intensified collagen deposition in the OIR group, which was similar to other BPD animal models. Combining the positive manifestations of HE staining, the rat OIR has potential in serving as a new model of BPD. 


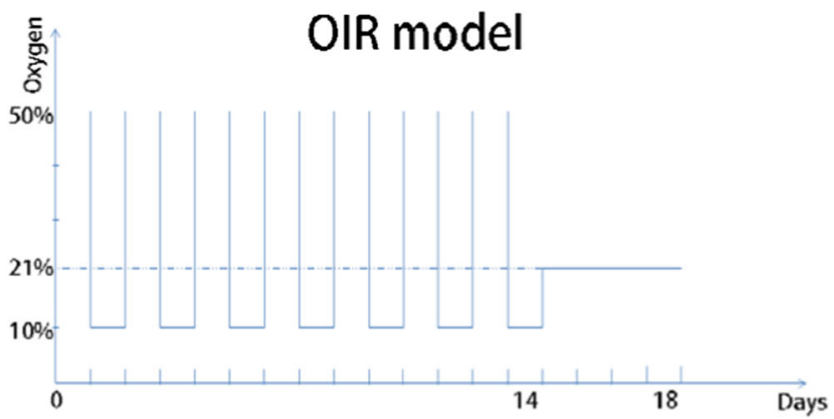

Control
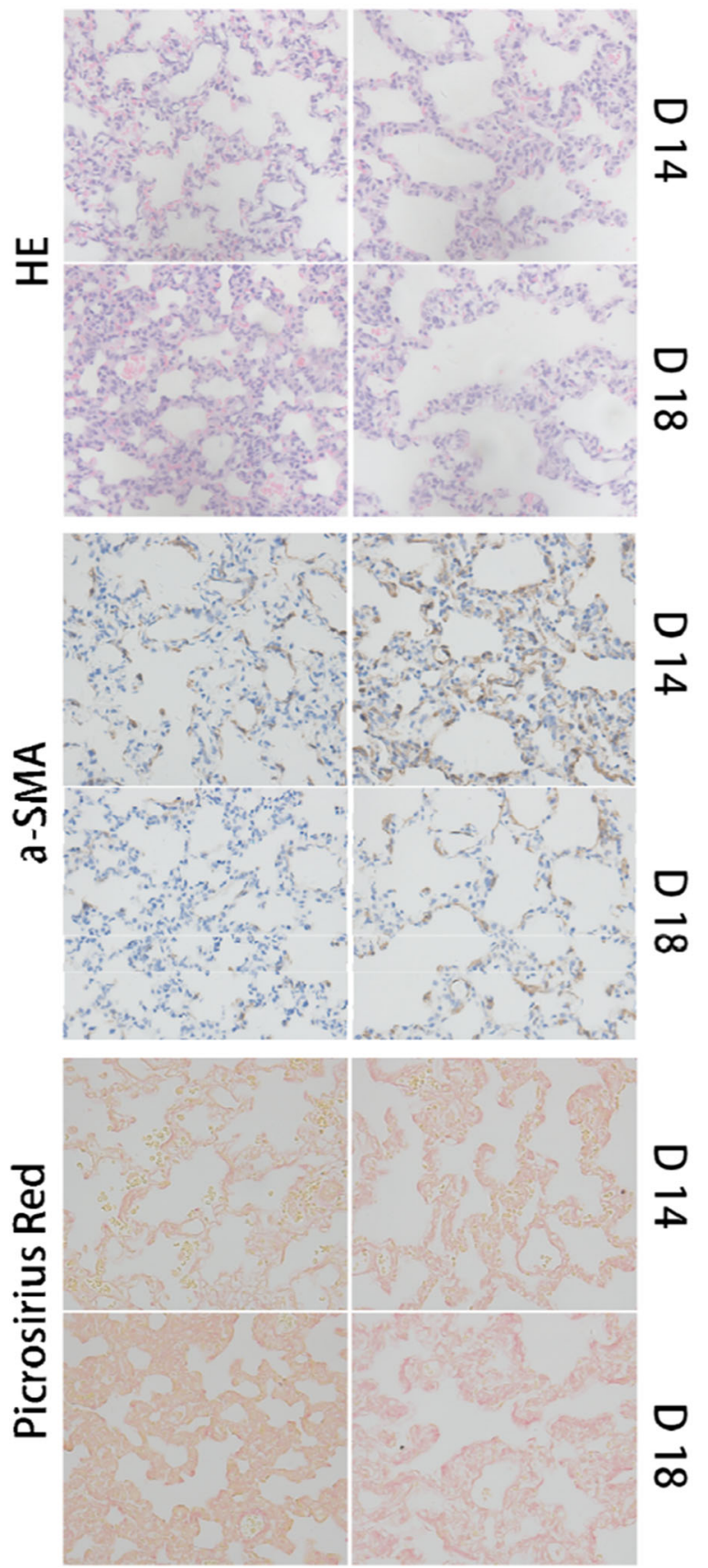

153

Neonatology

Caesarean Section is associated with reduced perinatal cytokine response, increased risk of bacterial colonization in the airway, and infantile wheezing

$\underline{\text { Sui-Ling Liao }}{ }^{1}$, Ming-Han Tsai ${ }^{1}$, Man-Chin Hua ${ }^{1}$, Shen-Hao Lai ${ }^{2}$, JingLong Huang ${ }^{2}$

${ }^{1}$ pediatrics, Chang Gung Memorial Hospital, Keelung branch, Taiwan

${ }^{2}$ Pediatrics, Chang Gung Memorial Hospital, Taiwan

\section{Background}

The relationship between cesarean section (CS) and allergic disorders such as asthma and wheezing has been inconsistent, and the mechanisms for their connection remained largely unknown. We aimed to investigate whether CS is associated with infantile wheeze and to explore the correlation between CS and several risk factors known to relate with development of allergy.

Method

Mononuclear cells were isolated from cord blood and assessed for TLR-triggered TNF-a, IL-6, and IL-10 responses by ELISA. Bacteria from nasopharyngeal specimens were identified with traditional culture methods at ages 1, 6, and 12 months. Infant lung function tests were performed at 6 and 12 months. Total and specific IgE, and clinical outcomes were assessed at 12 months of age.

\section{Results}

Children delivered by CS were associated with increased risk of wheezing (aOR: 1.89 ; $95 \%$ CI: $1.09-3.28$ ) and decreased compliance of the respiratory system at 12 months of age $(p=0.045)$. CS was associated with reduced perinatal TLR1-2- triggered TNFa and IL- 6 response ( $p=0.016$ for TNF- a and $p=0.026$ for IL6). Children born by CS had significantly higher bacteria colonization rate by 12 months of age (aOR: 1.89; $95 \% \mathrm{CI}$ : 1.11 3.20 ). No difference was found in the atopic sensitization or total IgE level between the 2 groups.

\section{Conclusion}

Our findings suggested that CS was associated with decreased pro-inflammatory cytokine response to TLR1-2 stimulation, followed by higher abundance of bacterial colonization in the airway during late infancy, thus increasing the risk of developing infantile wheezing.

379

Neonatology

Haemolytic disease of the newborn: a retrospective analysis of aetiology, hematologic evaluations and treatment in a tertiary neonatal centre.

Jana Lozar Krivec ${ }^{l}$, Metka Luznar ${ }^{1}$, Aneta Soltirovska-Šalamon ${ }^{l}$, Darja Paro-Panjan'

${ }^{1}$ Department of Neonatology, University Medical Centre Ljubljana, Division of Paediatrics, Slovenia

\section{Background}

Hemolytic disease of the newborn (HDN) results from immunologic destruction of erythrocytes (RBC), resulting in anaemia and hyperbilirubinemia. It is caused by maternal $\operatorname{IgG}$ antibodies that cross the placenta and in case of maternal/child blood group incompatibilities bind to RBC.

Objective

To evaluate the aetiology, hematologic findings and treatment of HDN. 


\section{Methods}

All patients with HDN, admitted to the Department of Neonatology, University Children's hospital Ljubljana between January 2007 and December 2016 were studied retrospectively. Inclusion criteria were laboratory sings of haemolysis (hyperbilirubinemia and anaemia or decrease in haemoglobin level or reticulocytosis) and positive Coombs test (DCT) or Lui elution. Medical records were reviewed and data of interest extracted. Patients with HDN due to incompatibility in: $\mathrm{ABO}$ blood groups (HDN-ABO), $\mathrm{RhD}$ (HDN-RhD) and other rarer blood antigens (HDN-other) were compered.

\section{Results}

During the study period 109 newborns with HDN were hospitalised. 92(84.5 \%) had HDN-ABO, 10(9 \%) HDN-RhD and $7(6.5 \%) \mathrm{HDN}$-other (Table 1). In HDN-ABO DCT was positive in 59(64\%) newborns. Heamoglobin level 10P for age was more often present in HDN-RhD (p0.05). Groups were comparable in the decrease in haemoglobin and reticulocytosis. Spherocyte were detected in $38 \%$ of HDN-ABO, while they were negative in all patients with HDN-RhD. Erythroblasts were more often detected in HDN-RhD and HDN-other than in HDN-ABO (36\% vs $60 \%$ vs $100 \%$ ). The differences in proportion of reticulocytes, number of erythroblasts and $\mathrm{COHb}$ level between the groups were not significant (Table 2). Exchange transfusion (ET) was performed in most patients with HDN-RhD and $41 \%$ with HDNABO (Table 3).

\section{Conclusions}

Most common cause of HDN was ABO incompatibility. 2/3 of patients with HDN-ABO had positive DCT, in others identification of antibodies was performed by Lui elution. Spherocytes were often identified in $\mathrm{HDN}-\mathrm{ABO}$ and proportion of patients with erythroblastosis was higher in HDN-RhD. Since our unit is referral tertiary centre, the proportion of newborns with HDN$\mathrm{ABO}$ who needed ET was high.

Table 1. Aetiology of the $\mathrm{HDN}$ in our patients.

\begin{tabular}{c|c|c}
\hline Antibodies & Number of children & Proportion \\
& $\mathbf{n}$ & $\%$ \\
\hline Anti-A & 67 & 61,5 \\
Anti-B & 25 & 22,9 \\
Anti-D & 6 & 5,5 \\
\hline Anti-C & 3 & 2,8 \\
\hline Anti-D in C & 4 & 3,7 \\
\hline Anti-c in E & 2 & 1,8 \\
Anti- Cw & 2 & 1,8 \\
\hline Total & 107 & 100 \\
\hline
\end{tabular}

Table 2. Comparison of hematologic evaluation results between patients with HDN-ABO, HDN$\mathrm{RhD}$ and HDN-other.

\begin{tabular}{|c|c|c|c|c|c|c|c|c|c|}
\hline & \multicolumn{2}{|c|}{ HDN-ABP } & \multicolumn{2}{|c|}{$\mathrm{HDN}-\mathrm{RhD}$} & \multicolumn{2}{|c|}{ HDN-other } & \multicolumn{2}{|c|}{ Total } & \multirow[t]{2}{*}{ Sign. } \\
\hline & $\mathrm{n}$ & $\%$ & $\mathrm{n}$ & $\%$ & $\mathrm{n}$ & $\%$ & $\mathrm{n}$ & $\%$ & \\
\hline $\begin{array}{l}\mathrm{Hb}<10 \mathrm{P} \\
\mathrm{n}=108\end{array}$ & 49 & 54 & 9 & 90 & 4 & 57 & 62 & 57 & ** \\
\hline $\begin{array}{l}\text { Decease in } \mathrm{Hb} \\
\mathrm{n}=105\end{array}$ & 48 & 54 & 5 & 56 & 3 & 43 & 56 & 53 & \\
\hline $\begin{array}{l}\text { Elevated Rte } \\
n=102\end{array}$ & 74 & 84 & 6 & 75 & 3 & so & 83 & $s I$ & \\
\hline $\begin{array}{l}\begin{array}{l}\text { Positive Sphero } \\
\mathrm{n}=93\end{array} \\
\end{array}$ & 30 & 38 & 0 & $o$ & 1 & 20 & 31 & 33 & \\
\hline $\begin{array}{l}\text { Positive Erythrob } \\
\mathrm{n}=94\end{array}$ & 30 & 36 & 3 & 60 & 3 & 100 & 36 & $3 s$ & \\
\hline $\begin{array}{l}\text { Positive DCT } \\
\mathrm{n}=107\end{array}$ & 59 & 65 & 10 & 100 & 6 & 100 & 75 & 70 & \\
\hline $\begin{array}{l}\mathrm{COHb}>1,7 \% \\
\mathrm{n}=107\end{array}$ & & 79 & 4 & 100 & 3 & 75 & 45 & so & \\
\hline Median \%Rtc & ${ }_{\min =1}$ & $\operatorname{lnax}=35$ & $(\min -$ & 6 & $(\min =$ & $\operatorname{nax}=13,2)$ & ${ }_{(\min =1}$ & $\operatorname{nax}=35$ & \\
\hline Median number Erythrob & ${ }_{(\min =0}$ & $2 x=250)$ & $(\min -$ & $\begin{array}{l}5 \\
\max -19)\end{array}$ & & 5 & ${ }_{(\min =0}$ & $2 x=250)$ & \\
\hline Avera ge COHb (SD) & & & & $(0,35)$ & & & & & \\
\hline
\end{tabular}

Table 3. Therapy of HDN in our patients.

\begin{tabular}{|c|c|c|c|c|c|c|c|c|}
\hline \multirow[t]{2}{*}{ Therapy } & \multicolumn{2}{|c|}{$\begin{array}{c}\text { HDN-ABO } \\
\text { n (\%) }\end{array}$} & \multicolumn{2}{|c|}{$\begin{array}{c}\text { HDN-RhD } \\
\text { n (\%) }\end{array}$} & \multicolumn{2}{|c|}{$\begin{array}{c}\text { HDN-other } \\
\text { n (\%) }\end{array}$} & \multicolumn{2}{|c|}{$\begin{array}{l}\text { Total } \\
\text { n (\%) }\end{array}$} \\
\hline & 3 & 3,5 & 0 & & 1 & 14 & 4 & 3,7 \\
\hline Photo & 46 & so & 0 & & 4 & 57 & 50 & 46 \\
\hline Photo + ET & 38 & 41,5 & 8 & 80 & 2 & 29 & 48 & 44 \\
\hline Photo + RBC trans & 2 & 2 & 0 & & 0 & & 2 & $1, s$ \\
\hline RBC trans & 2 & 2 & 0 & & o & & 2 & $1, s$ \\
\hline Photo+ RBC trans +EPO & 1 & 1 & 0 & & 0 & & 1 & 0,9 \\
\hline IUT + Photo & 0 & & 1 & 10 & 0 & & 1 & 0,9 \\
\hline IUT + RBC trans & 0 & & 1 & 10 & 0 & & 1 & 0,9 \\
\hline Total & 92 & 100 & 10 & 100 & 7 & 100 & 109 & 100 \\
\hline
\end{tabular}

384

Neonatology

Neonatal alloimmune thrombocytopenia: a retrospective analysis of clinical and laboratory findings in Slovenian patients Jana Lozar Krivec ${ }^{1}$, Tatjana Tanasic ${ }^{1}$, Jelena Đorđević ${ }^{1}$, Petra Fidler ${ }^{2}$, Irena Štucin Gantar ${ }^{3}$, Andreja Trojner-Bregar ${ }^{3}$, Primož Rožman ${ }^{4}$, Polona Klemenc ${ }^{4}$, Darja Paro-Panjan ${ }^{1}$

${ }^{1}$ Department of Neonatology, University Medical Centre Ljubljana, Division of Paediatrics, Slovenia

${ }^{2}$ Department of Perinatology, University Medical Centre Maribor, Division of Gynecology and Perinatology, Slovenia

${ }^{3}$ Department of Gynecology and Obstretics, University Medical Centre Ljubljana, Division of Perinatology, Slovenia

${ }^{4}$ Platelet Immunology Laboratory, Blood Transfusion Centre of Slovenia, Slovenia

\section{Background}

Neonatal alloimmune thrombocytopenia (NAIT) results from transplacental transmission and binding of alloimmune antibodies on child's platelets (PLT) antigens. It is a rare disease with unpredictable course.

\section{Objectives}

To evaluate the incidence, aetiology, clinical course and adherence of recommendations for treatment of NAIT in Slovenia.

\section{Methods}

Medical documentation of newborns with NAIT born in Slovenia between 1996 and 2016 was studied. Cases were identified from the Slovenian Blood transfusion centre database. NAIT inclusion criteria were neonatal thrombocytopenia and the presence of PLT antibodies (PLT-Ab) in mother and/or newborn. 


\section{Results}

PLT-Ab were detected in 65 out of 229 referrals (due to neonatal thrombocytopenia, recurrent miscarriage or intrauterine fetal death). Sixteen medical records of the newborns were not available. Thirty-seven from the remaining 49 with PLT-Ab developed thrombocytopenia (incidence $0.1 / 1000$ live births). Specificity of PLT-Ab in Slovenian patients is shown in Table $1.15(38 \%)$ of the newborns were anti-HPA-1a positive and $8(21 \%)$ were HLA antibodies positive only. Five mothers needed IVIG prenatally. Two out of 6 mothers with the history of NAIT delivered with C-section. Twenty-seven newborns presented with petechiae and/or suffusions and one with intraventricular haemorrhage. Among 9 asymptomatic newborns, 2 had anti-HPA-1a. PLT were lower in newborns with anti-HPA-1a with the lowest value presented earlier (2nd day vs $3 \mathrm{rd} / 4$ th day) than in the other groups (Figure 1). Eight $(20.5 \%$ ) newborns needed PLT transfusion (PLT-trans), 4 (10 \%) PLT-trans and IVIG, $1(2.5 \%)$ IVIG, and $1(2.5 \%)$ PLT-trans, IVIG and steroids.

Conclusion

The most common aetiology of NAIT was anti-HPA-1a but its frequency was lower than reported in the literature. HLA antibodies were frequently identified as the only antibodies in patients with symptomatic thrombocytopenia. Anti-HPA-1a caused more severe thrombocytopenia than other PLT-Ab. Only $35 \%$ of newborns with PLT $30 \times 10^{9} / \mathrm{L}$ received therapy and C-section was not performed in all cases with history of NAIT.

Table 1. Specificity of platelet's antibodies in patients with NAIT in Slovenia.

\begin{tabular}{|c|c|c|}
\hline A ntibodies & $\begin{array}{c}\text { Number of patients } \\
n\end{array}$ & $\begin{array}{c}\text { Proportion } \\
\%\end{array}$ \\
\hline HPA1 a & 11 & 28 \\
\hline HPA15ab & 1 & 2.5 \\
\hline FPA33 & 1 & 2.5 \\
\hline Anti gplib/11la & 2 & 5 \\
\hline FPAIa $+\mathrm{HLA}$ & 4 & 10 \\
\hline HPASB+FILA & 2 & 3 \\
\hline HLA. & 8 & 21 \\
\hline Unspecified & 10 & 26 \\
\hline Total & 39 & 100 \\
\hline
\end{tabular}

Figure 1. Distribution of patients with different level of thrombocytopenia according to platelet's antibody type. (PLT- platelets, HPA1 a - human platelet antigen 1a, AB - antibody)

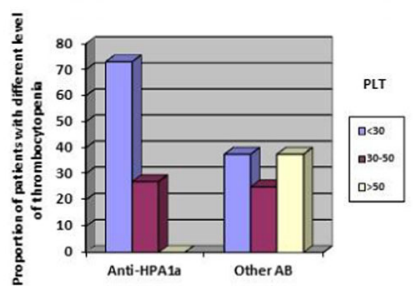

670

\section{Neonatology}

Validity of Ages and Stages Questionnaires Social Emotional-2 at predicting Bayley III Social Emotional and Adaptive Behavioural results in children with Congenital Diaphragmatic Hernia Aine Lynch ${ }^{1}$, Stephanie Hardcastle ${ }^{2}$, Aine Sutton ${ }^{2}$, Eleanor Molloy ${ }^{1,3}$, John Kelleher

${ }^{1}$ Department of Neonatology, Our Lady's Children's Hospital, Crumlin, Ireland ${ }^{2}$ Department of Psychology, Our Lady's Children's Hospital, Crumlin, Ireland

${ }^{3}$ Paediatric School of Medicine, Trinity College Dublin, Ireland

\section{Background}

Congenital diaphragmatic hernia $(\mathrm{CDH})$ is a rare congenital malformation affecting 1/3000 live births. Neurodevelopmental delay in survivors ranges from $16 \%$ to $70 \%$.

\section{Objective}

To examine the correlation between Ages and Stages Questionnaires Social Emotional - 2 (ASQ:SE-2) and Bayley Scales of Infant and Toddler Development III Social-Emotional and Adaptive Behaviour Questionnaire (BSID III SE/ABQ) results in a cohort of children with $\mathrm{CDH}$.

\section{Methods}

This was a singe centre prospective cohort study of neurodevelopmental outcomes of patients with $\mathrm{CDH}$ at 24-36 months corrected age. The primary outcome was correlation of social emotional and adaptive behavioural outcomes using two standardised assessments. Data was analysed using Microsoft Excel.

Standard scores greater than 2 standard deviations (SD) below mean on BSID III SE/ABQ were considered a "fail" and scores between 1 and 2 $\mathrm{SD}$ of the mean were considered "borderline".

A score indicating "refer" in the ASQ:SE-2 was considered a "fail" and a score indicating "monitor" was considered "borderline".

\section{Results}

Thirty-one patients were identified as eligible for study inclusion. Of these, 8 had died at the time of the study, 23 were approached and 20 consented. Sensitivity of ASQ:SE-2 for predicting results of BSID III SE/ABQ was $50 \%$ (PPV $=67 \%)$; with specificity $94 \%$ (NPV $=88 \%$ ) using referral cut-offs. When 65th percentile (monitoring zone) was used as cut off, sensitivity of ASQ:SE-2 for predicting results of BSID III SE/ABQ was $33.3 \%$ $(\mathrm{PPV}=75 \%)$; with specificity $91 \%(\mathrm{NPV}=62.5 \%)$

\begin{tabular}{|c|c|c|c|}
\hline \multirow[b]{2}{*}{ ASQ SE-2 } & \multicolumn{3}{|c|}{ BSID III SE/ABQ } \\
\hline & PASS & FAIL & TOTALS \\
\hline PASS & 15 & 2 & 17 \\
\hline FAIL & 1 & 2 & 3 \\
\hline TOTALS & 16 & 4 & 20 \\
\hline
\end{tabular}

Table 2: sensitivity/specificity of the AQ:SE-2 $\left(>65^{\text {th }}\right.$ percentile) and Bayleys III Social-Emotional and Adaptive Behaviour Questionnaire (>1 SD from mean)

\begin{tabular}{|c|c|c|c|}
\hline & BSID III SE/ABQ & & \\
\hline ASQ SE-2 & PASS & BORDERLINE/FAIL & TOTALS \\
\hline PASS & 10 & 6 & 16 \\
\hline BORDERLINE/FAIL & 1 & 3 & 4 \\
\hline TOTALS & 11 & 9 & 20 \\
\hline
\end{tabular}

Sensitivity $33.3 \%$ (PPV = 75\%); Specificity 91\% (NPV = 62.5\%)

\section{Conclusions}

In this cohort, sensitivity and specificity of ASQ:SE-2 did not meet quoted levels of 78-88\%. Sensitivity was less when cut off scores of 1 SD was used. This indicates that ASQ:SE-2 does not have appropriate correlation with BSID III SE/ABQ to be used to predict outcomes in this cohort.

\section{7}

\section{Neonatology}

The Effect of Gestational Diabetes on Human Milk Macronutrients Content Dana Shapira ${ }^{1}$, Ronit Lubetzky ${ }^{1,4}$, Francis Mimouni $^{3}$, Dror Mandel ${ }^{2,4}$

${ }^{I}$ Pediatrics, Dana Dwek Children's Hospital, Tel Aviv Sourasky Medical Center, Israel

${ }^{2}$ Neonatology, Dana Dwek Children's Hospital, Tel Aviv Sourasky Medical Center, Israel

${ }^{3}$ Neonatology, Shaare Zedek Medical Center, Israel

${ }^{4}$ Sackler Faculty of Medicine, Tel Aviv University, Israel 


\section{Background}

Little is known about the effect of gestational-diabetes (GD) upon macronutrients content of human-milk (HM).

Aim

To study macronutrients (fat, lactose, protein) and caloric content in HM from women with GD compared to women with no-GD.

Methods

Sixty-two lactating mothers ( 31 with GD, 31 without GD) were recruited. Diagnosis of GD was made by using a 100-gram Glucose-Tolerance-Test. After manual expression, each mother contributed 3 samples of HM (during first 72 hours after labor (colostrum), after 7-days (transitional-HM) and at 14-days post-partum (mature-HM). Immediately following expression, samples were stored at $-20{ }^{\circ} \mathrm{C}$ until thawed and analyzed using infrared transmission spectroscopy HM analyzer.

Results

Sixteen women $(52 \%)$ in the GD group were treated by diet alone (48\%) while $15(48 \%)$ by pharmacotherapy. The non-GD and GD groups did not differ in terms of maternal age, maternal pre pregnancy weight, height, diet and weight gain during pregnancy, gestational age and infant birthweight. A total of $186 \mathrm{HM}$ milk samples were collected. Macronutrients content in colostrum and transitional milk did not differ between the 2 groups. Fat and energy contents in mature HM were higher in the non-GD samples than in the GD samples ( $p=0.07$ and 0.02 , respectively). There were no differences in macronutrients content of samples of mother with diet-treated-GD compared to mother with pharmacotherapy-treated-GD.

\section{Conclusions}

Fat and energy content of mature milk obtained from mothers with GD is lower compared to that of milk from mothers without GD. The mechanism and biological significance of our findings is yet to be determined.

762

\section{Neonatology}

When are "Sticky Eyes" a Neonatal Emergency? Neonatal Gonococcal Conjunctivitis: Case Report and Literature Review Karen McCarthy ${ }^{1}$, Nese Aber ${ }^{1}$, Idress $^{2}$, T Ryan ${ }^{l}$

${ }^{T}$ Cork University Maternity Hospital, Department of Neonatology, Ireland

${ }^{2}$ Cork University Hospital, Department of Opthalmology, Ireland

\section{Background}

Opthalmia neonatorum as a result of Neisseria Gonorrhoeae infection is rarely encountered in modern neonatal practice. It is, however, a sight threatening medical emergency and an awareness of this pathogen, the consequences of infection and up to date management strategies is paramount. We present the first reported case from a maternity hospital in Ireland.

\section{Objective}

The objective of this report was to describe a case of neonatal gonococcal conjunctivitis and review the literature relating to this condition.

\section{Case Report}

We present the case of a 2 day old term infant who was noted to have bilateral purulent exudate and periorbital oedema, more pronounced on the right side. An ophthalmological assessment revealed right haemorrhagic conjunctivitis and left conjunctivial injection with no corneal involvement or evidence of endopthalmitis. A history of paternal genital infection requiring antibiotics during pregnancy was given, however the infant's mother was not investigated or treated. Investigations included eye swabs for culture and sensitivity, Chlamydia and Gonococcus PCR. Empiric treatment was commenced with IV Cefotaxime, PO Azithromycin and Chloromycetin ophthalmic drops. Neisseria Gonorrhoeae was grown and RT-PCR was also positive for $N$. Gonorrhoeae. Antibiotic therapy was rationalized and the baby received 1 dose of IV Ceftriaxone and topical Azithromycin. Clinical improvement was noted within 12 hours of initial therapy and had completely resolved by 48 hours.

\section{Conclusion}

The incidence of gonorrhea notifications has been increasing among the Irish population. From 2009 to 2014 there was a $200 \%$ increase in notifications. This reflects European trends where there was a $19 \%$ increase in gonorrhea cases between 2013 and 2014 with $55 \%$ of cases occurring in the young heterosexual population. Potential consequences of gonococcal infection include keratitis, endopthalmitis, globe perforation and visual loss. An awareness of this serious neonatal complication is necessary as prompt recognition and treatment are sight saving interventions.

\section{5}

\section{Neonatology}

Randomized Control Trial to Compare Kangaroo Mother Care with Oral Sucrose for Pain Management in Premature Neonates on heel prick Vivek Shukla ${ }^{2}$, Apurva Chapla ${ }^{1}$, Jigar Uperiyal, Archana Nimbalkar ${ }^{3}$, Ajay Phatak', Somashekhar Nimbalkar ${ }^{1,2}$

${ }^{1}$ Department of Pediatrics, Pramukhswami Medical College, India

${ }^{2}$ Central Research Services, Charutar Arogya Mandal, India

${ }^{3}$ Department of Physiology, Pramukhswami Medical College, India

\section{Background}

Multiple studies have shown the benefits of Kangaroo mother care (KMC) and oral Sucrose on neonatal pain reduction. There is lack of head to head randomized control trials comparing the pain reduction between these two methods. Objectives To compare efficacy of Kangaroo Mother Carewith oral sucrose for pain management on heel prick with parallel group randomized control trial.

\section{Methods}

Preterm neonates (28-36 weeks gestational age) were recruited. Study interventions were done on neonates requiring heel prick as per medical management. Fifty participants per study arm were randomized to (1) Kangaroo Mother Care (KMC) group and (2) Sucrose groupwith the use of WINPEPI software. KMC was provided for $10 \mathrm{~min}$ in advance to procedure and continued till at least $5 \mathrm{~min}$ post procedure. Infants less than 32 weeks corrected age received $0.5 \mathrm{ml}$ of sucrose and those between 32 to 36 weeks received $1 \mathrm{ml}$ sucrose 2 minutes prior to the procedure. Blinded assessment of pain was done using Premature Infant Pain Profile score on recorded videos. Trial was registered on CRTI with CTRI/2017/ 02/007824.

\section{Results}

Analysis of 100 (50 KMC and 50 Sucrose group) cases were done.The mean(SD) gestational age [32.79(2.34)] weeks, age of neonate [14.04(11.10)] days and birth weight [1.62(0.35)] Kg. was similar across groups. The difference across components of PIPP is seen in Figure 1.

\begin{tabular}{|l|l|l|l}
\hline Particulars & KMC Group & \multicolumn{1}{l|}{ Sucrose Group } & \multirow{2}{*}{-value } \\
\cline { 1 - 2 } & Mean(SD) & Mean(SD) & \\
\hline GESTATIONAL AGE & $1.46(0.61)$ & $0.8(0.53)$ & $<0.001$ \\
\hline BEHAVIOURAL STATE & $2.58(0.76)$ & $1.34(1.17)$ & $<0.001$ \\
\hline Heart Rate & $0.42(0.5)$ & $1.4(0.61)$ & $<0.001$ \\
\hline SPO 2 & $0.24(0.48)$ & $0.24(0.43)$ & 1 \\
\hline BROW BULGE & $0.52(0.71)$ & $0.94(0.79)$ & 0.006 \\
\hline EYE SQUEEZE & $1.5(0.93)$ & $1.86(0.97)$ & 0.06 \\
NASO LABIALFURROW & $1.02(0.89)$ & $1.52(0.91)$ & 0.007 \\
\hline TOTAL PIPP SCORE & $7.74(2.43)$ & $8.1(2.82)$ & 0.50 \\
\hline
\end{tabular}

Mean(SD) PIPP score was smaller in KMC group as compared to Sucrose group but could not achieve statistical significance [7.74(2.43) vs. 8.1(2.82), $p=0.50)$. [Figure 2]. 


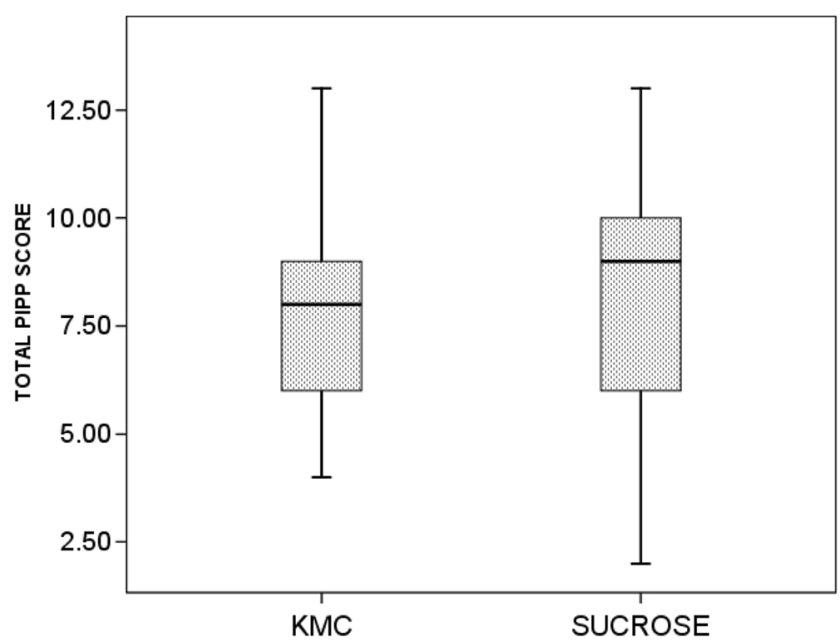

\section{Conclusion}

There is no difference in between the pain reducing possibilities of sucrose and KMC and hence they can used alternatively as the situation demands. KMC should be preferred if possible due to other beneficial effects but sucrose is a good substitute when $\mathrm{KMC}$ is not feasible.

\section{2}

\section{Neonatology}

Post Discharge Practice of Kangaroo Mother Care at Home in Anand, Gujarat, India

Somashekhar Nimbalkar $^{1,2}$, Ruju Doshi ${ }^{1}$, Vallaree Morgaonkar ${ }^{1}$, Dipen Patel $^{1}$, Ajay Phatak ${ }^{2}$, Archana Nimbalkar ${ }^{3}$

${ }^{1}$ Department of Pediatrics, Pramukhswami Medical College, India

${ }^{2}$ Central Research Services, Charutar Arogya Mandal, India

${ }^{3}$ Department of Physiology, Pramukhswami Medical College, India

\section{Background}

Kangaroo Mother Care (KMC) prevents/decreases hypothermia in neonates, with multiple other benefits and is a high impact intervention to reduce neonatal mortality. KMC is initiated in hospitals following delivery and is continued at home after discharge for better gains.

Objective

To evaluate KMC practices at home among LBW and premature infants that began receiving $\mathrm{KMC}$ in the hospital.

\section{Methods}

We surveyed mothers of babies admitted to NICU, who completed treatment and were advised to continue $\mathrm{KMC}$ at home. Medical record and contact details of neonates $(N=97)$ that were admitted from Jan 2016 to Oct 2016 were obtained. Mothers were phoned and interviews were scheduled at home. Results

Of 120 mothers, 70 participated. 60(85.7\%) neonates were preterm and mean(SD) discharge weight was $1.8(0.3) \mathrm{Kg}$. Mean (SD) age of mothers was 25.1(3.3) y. Most mothers 69(98.6) were explained benefits and method of KMC through charts, many 62(88.6) received reinforced advice during outpatient visits and most 68(97.1) reported practicing $\mathrm{KMC}$ at home. However, 39(55.7 \%) mothers provided KMC for $6 \mathrm{~h}$ per day. In 42(60 \%) cases, $\mathrm{KMC}$ was provided by other family member, 48(68.6\%) said they had family support for daily work. There was maternal fatigue and pain [56(80\%)], lack of house help support [48(68.6\%)], and presence of sibling to look after [38(54.3\%)]. Majority were motivated to provide KMC [62(88.6\%)] and would advise other mothers for the same [69(98.6\%)].

\section{Conclusion}

Mothers were supportive and provided KMC at home following discharge but quality is suboptimal. Interventions focussed on improving $\mathrm{KMC}$ at home need to be explored urgently to extract maximum benefit of this proven intervention.

466

Neonatology

Embrace $^{\text {TM }}$ Versus Conventional Care during Transport of Newborn 1500 Grams: A Randomized Double Blind Controlled Trial at Tertiary Care Centre In Gujarat, India

Vallaree Morgaonkar ${ }^{1}$, Dipen Patel ${ }^{1}$, Archana Nimbalkar ${ }^{2}$, Ajay Phatak ${ }^{3}$, Somashekhar Nimbalkar ${ }^{1,3}$

${ }^{1}$ Department of Pediatrics, Pramukhswami Medical College, India

${ }^{2}$ Department of Physiology, Pramukhswami Medical College, India

${ }^{3}$ Central Research Services, Charutar Arogya Mandal, India

\section{Background}

Neonates are prone to develop hypothermia during transport especially in resource poor settings. The Embrace ${ }^{\mathrm{TM}}$ is a small sleeping bag-like apparatus. The Embrace ${ }^{\mathrm{TM}}$ are composed of three components: an infant-sized sleeping bag or baby interface, a pouch of phase change material $(\mathrm{PCM})$, and a heater. Embrace ${ }^{\mathrm{TM}}$ may be used to prevent neonatal hypothermia during transport.

Objective

We decided to assess effectiveness of Embrace ${ }^{\mathrm{TM}}$ vs conventional care during transport from emergency department (ED) to Neonatal Intensive Care Unit (NICU).

\section{Methods}

All neonates weighing 1500 grams coming to ED were included. During stay in ED, neonates were placed in open warmers. Neonate was placed in Embrace $^{\mathrm{TM}}$ or Pre-Warmed linen (Control) for transport to NICU. The embrace/linen was removed and neonate was placed on radiant warmer in the NICU. Temperature was recorded again ( 0 hour) and at $0.25,0.5,1,2$, $3,6,12$ and 24 hours of the NICU stay. The distance travelled by every neonate from the ED to NICU was 700 meter.

\section{Results}

Socio-demographic, clinical variables were similar. Preterm were 31/60 (35 \%) in Embrace ${ }^{\mathrm{TM}}, 19 / 60$ (31.67 \%) in control. Females were 21/60 $(35 \%)$ in both groups. Mean birth weight was 2.5(embrace)2.44(control). Average age at admission is 4.9 days(embrace) 3.15 (control). Average time for transport was 12.3 mins(embrace) and 13 mins(control). From ED to $\mathrm{NICU}$, the mean $(\mathrm{SD})$ temperature difference in EMBRACE ${ }^{\mathrm{TM}}$ group was $+0.37 \mathrm{C}(0.53)$ (that is the temperature increased by +0.37 degree) whereas the same was $-0.37(0.79)$ (that is the temperature dropped by 0.37 degrees on an average). This difference was statistically significant ( $\mathrm{p} 0.001)$.

\section{Conclusions}

Embrace $^{\mathrm{TM}}$ is effective in maintaining euthermia in intervention group as compared to control group for short distance neonatal transport. More studies are required to assess effectiveness for inter hospital transfers of prolonged duration/distance (few $\mathrm{Km}$ ).

237

Neonatology

Salivary C-Reactive Protein as Non-Invasive Marker in Diagnosis of Neonatal Sepsis

Ahmed Omran $^{1}$, Abdallah Maaroof ${ }^{1}$, Mai Saleh ${ }^{2}$, Amina Abdelwahab ${ }^{1}$

${ }^{T}$ Departments of Pediatrics \& Neonatology, Faculty of Medicine, Suez Canal University, Egypt

${ }^{2}$ Department of Clinical Pathology, Faculty of Medicine, Suez, Canal University, Egypt

\section{Background}

Neonatal sepsis is a major cause of morbidity and mortality in neonatal intensive care units. Neonatal saliva overcomes many of the hurdles associated with neonatal research and offers the investigator a new and exciting non-invasive sample source for 
exploring neonatal biology. Till now salivary C-reactive protein (CRP), neutrophil/lymphocyte ratio (NLR) and platelets/ lymphocytes ratio (PLR) have not been studied as markers of diagnosis in neonatal sepsis.

\section{Objective}

To assess the applicability of salivary CRP, mean platelet volume (MPV), NLR and PLR in diagnosis of neonatal sepsis.

\section{Methods}

Prospective case control study of 70 full term neonates, 35 with sepsis ( 20 with proven sepsis and 15 with clinical sepsis) and 35 healthy controls were enrolled. Serum and salivary CRP concentrations were measured by ELISA, while MPV, NLR and PLR were measured by automated blood cell counter.

\section{Results}

This study showed statistically significant difference between salivary CRP mean between septic neonates and controls $(12.0 \pm 4.6 \mathrm{ng} / \mathrm{L}$ and $2.8 \pm 1.2 \mathrm{ng} / \mathrm{L}$ ) respectively. At cut- off point $3.48 \mathrm{ng} / \mathrm{L}$ salivary CRP showed sensitivity $94.3 \%$ and specificity $80 \%$. Salivary CRP showed also good predictive accuracy in predicting elevated serum CRP values in septic neonates. MPV and NLR showed significant difference between septic neonates and controls, $(10.2 \pm 1.2 \mathrm{fL} / 8.0 \pm 0.5 \mathrm{fL}),(2.9 \pm 1.7,1.6 \pm 0.4)$ respectively. MPV at cut-off point $10.2 \mathrm{fL}$ has $80 \%$ sensitivity and specificity. NLR at cut-off point 2.7 has $80 \%$ sensitivity and $57.1 \%$ specificity.

\section{Conclusion}

This is the first study to demonstrate that salivary CRP and NLR are suitable as diagnostic markers in neonatal sepsis. Salivary CRP could be used as a non-invasive marker for diagnosis of neonatal sepsis.

\section{0}

\section{Neonatology}

Outcomes in East London Premature Babies on Home Oxygen: Growth and Readmissions

Raymand Pang ${ }^{l}$, Harriet Williams-Gunn ${ }^{1}$, Prita Rughani ${ }^{l}$, Ajay Sinha ${ }^{l}$, Cristina Ilea ${ }^{I}$, Ghada Saleh ${ }^{I}$, Michael Hird ${ }^{l}$, Caroline May ${ }^{I}$

${ }^{1}$ Neonatal Unit, Royal London Hospital, Barts Health NHS Trust, UK

\section{Background}

Preterm infants with bronchopulmonary dysplasia are a vulnerable group of babies. Home oxygen has the advantage of early discharge to allow family life normalisation, hopefully facilitating growth. We carried out a study to evaluate the growth and readmission rates.

\section{Method}

Data was collected retrospectively with electronic patient records for infants born at the Royal London Hospital between 20112014, discharged on home oxygen and followed up in clinic until 2 years old. Growth parameteric SD scores (SDS) were calculated using LMS calculator for Microsoft Excel and data was analysed with non-parametric tests due to the non-normal distribution of the data from small sample size.

\section{Results}

Thirty-six babies met our criteria, born between $22+6$ and 30 weeks gestation. Babies less than 26 weeks spent longer on the ventilator (median $=36$ days) compared to babies born at 26 weeks or more (median 6 days, p0.001). Already small at birth (median SDS-0.68, $25^{\text {th }}$ centile), growth is poor during the first 28 days on NICU (median SDS-1.74, $4^{\text {th }}$ centile) then remains steady at discharge $\left(6^{\text {th }}\right.$ centile, median SDS-1.53). Following discharge, half of the babies required at least 1 Accident and Emergency attendance within the first year, particularly if discharged over autumn or winter (p0.015). Half of the episodes resulted in admissions but very few required intensive care $(n=4)$. Growth improved rapidly after discharge, catching up to their birth centile at 4 months corrected (median SDS- 0.63 , 26th centile). By 1 year corrected, growth velocity tailed off (median SDS-0.93, $18^{\text {th }}$ centile), although still above the discharge centile.

\section{Conclusion}

Growth fell dramatically within the first month, which may reflect their unstable health. Home oxygen leading to normalisation of family life may contribute to rapid growth in the first few months but may also contribute to increased anxiety resulting to unnecessary A\&E visits. Improved community support may reduce the burden on acute services.
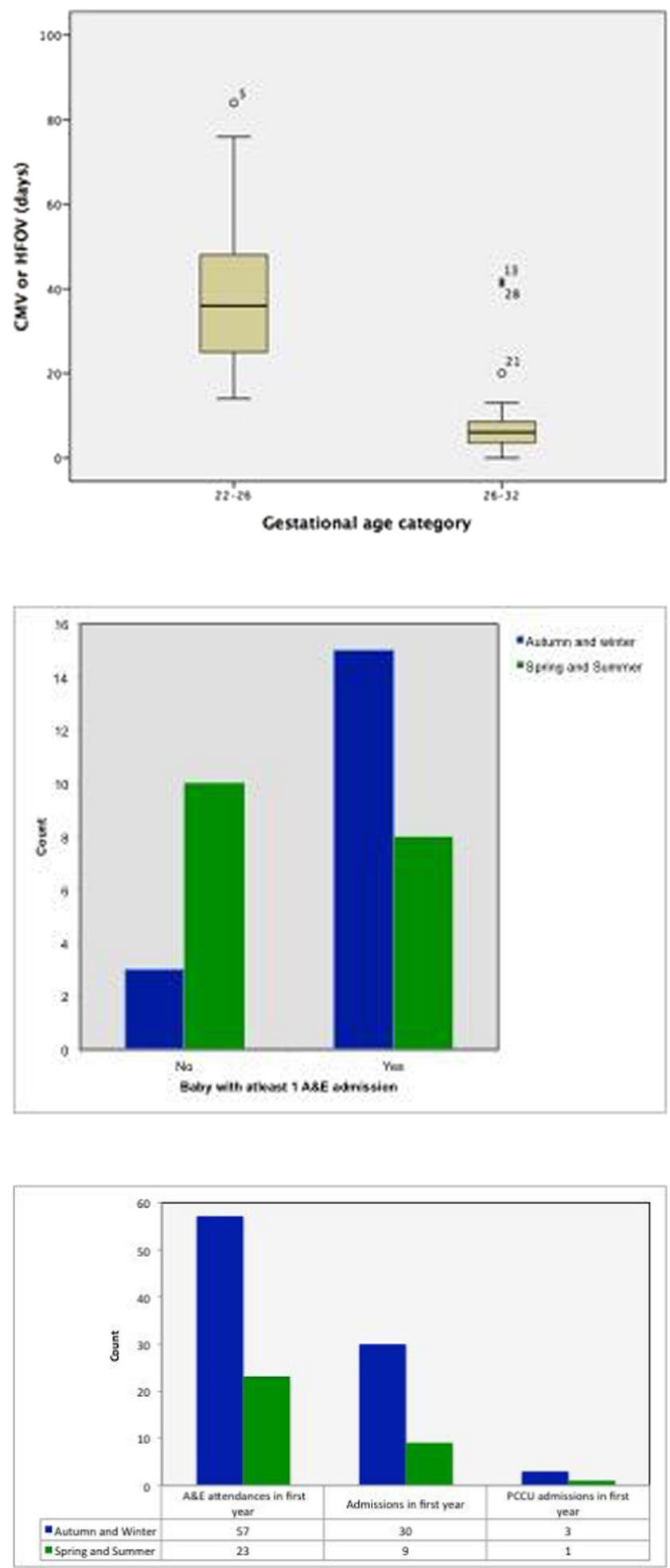
Growth of babies during NICU admission and subsequent discharge on Home oxygen (median Centile)

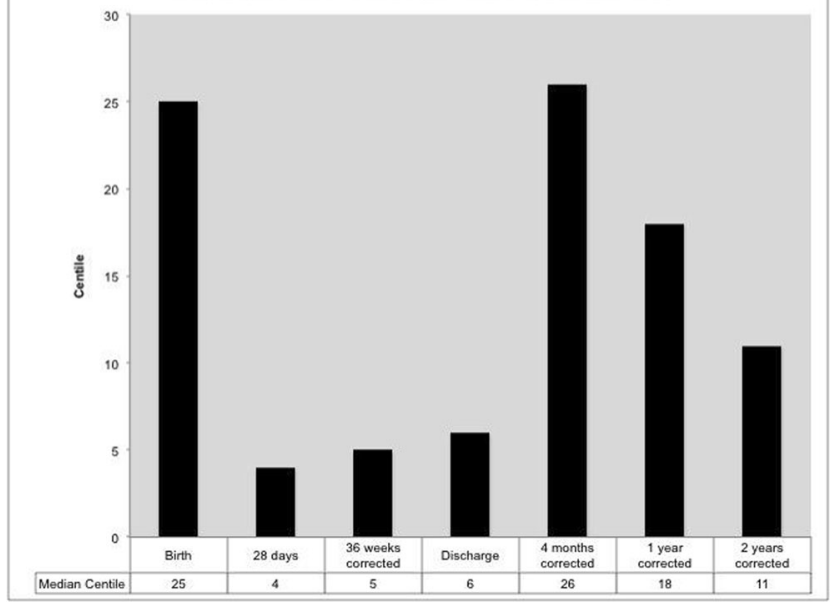

267

\section{Neonatology}

The Neonatal Difficult Airway Course - a collaboration between three Teaching Hospitals in the UK Abby Parish ${ }^{1}$, Torsten Hildebrandt ${ }^{2}$, Alok Sharma ${ }^{3}$

${ }^{T}$ Neonatal Intensive Care Unit, Cardiff and Vale University Health Board, UK

${ }^{2}$ Paediatrics, Abertawe Bro Morgannwg University Health Board, UK

${ }^{3}$ Neonatal Intensive Care Unit, University Hospital Southampton NHS Foundation Trust, UK

\section{Background}

Encountering a difficult airway in a neonate is a life-threatening emergency. Contrary to practice in older children there are no national guidelines available in the UK to facilitate a structured approach. The course was initiated at the Princess Anne Hospital Southampton and rolled out through collaboration with the University Hospital of Wales Cardiff and Abertawe Bro Morgannwg University Healthboard.

\section{Objectives}

The course focuses on assessment and decision making for neonatologists faced with a difficult airway.

Methods

The course is designed in a multidisciplinary fashion to give neonatal, surgical and anaesthetic perspectives involving lectures from ear, nose and throat surgeons (ENT), Anaesthetists and Neonatologists to provide background information about anatomy, common problems and practical approaches. There is a series of workshops to allow participants to practise skills with video-assisted technology (GlideScope®, Storz CMAC®, InfantView from ACUTRONIC), laryngeal masks and i-gel®. ENT surgeons demonstrate surgical equipment and discuss surgical airways. Neonatologists demonstrate the use of a difficult airway trolley and guidelines in clinical practice. The second part of the day consists of simulation training with small groups rotating through 4 scenarios to put learning into practice.

\section{Results}

The course has run 5 times in involved centres in the UK, with plans to run more courses in other locations. Feedback is consistently high with over $93 \%$ of participants rating the course as good or excellent and over $93 \%$ of participants agreeing that the course was effective and would change their clinical management. A thematic analysis of comments concluded the participants would 'highly recommend' and it was 'helpful' with 'realistic' methodology.

Conclusions

We believe The Neonatal Difficult Airway Course has demonstrated its usefulness with persistently very good feedback and hope that it will give attendees a frame work to deal with this difficult clinical scenario in a structured and logical way.

\section{9}

\section{Neonatology}

Review of neonatal pneumothoraces in tertiary neonatal units in Wales Prasad Parvathamma ${ }^{1}$, Vasiliki Makri ${ }^{3}$, Gail Tector ${ }^{2}$, Joanna Webb ${ }^{3}$, Mallinath Chakraborty $^{2}$, Gautam Bagga ${ }^{1}$

${ }^{1}$ Neonatalogy, Royal Gwent Hospital, UK

${ }^{2}$ Neonatalogy, University Hospital of Wales, UK

${ }^{3}$ Neonatalogy, Singleton Hospital, Swansea, UK, UK

\section{Background}

Pneumothorax is the most common air-leak syndrome resulting in significant morbidity and mortality in neonates.

We aimed to evaluate common clinical characteristics, any identifiable predisposing factors in neonates developing pneumothorax.

\section{Methods}

We retrospectively reviewed the medical records of all the neonates with pneumothorax admitted in 3 NICUs in Wales from January 2012 to December 2014.

\section{Results}

Full clinical data was available in 83 neonates. More common in males $66.2 \%$ and most of the neonates were inborn (70 \%). Thirty-two $(38.5 \%)$ were 34 weeks' Gestation, 12 borderline preterm $(14.4 \%)$ and 39 term babies (47\%). About $78 \%$ of eligible babies (42) received atleast one dose of antenatal steroids. Fifty neonates $(60 \%)$ required resuscitation at 5 mins (42) or more (9). Forty-six babies were intubated within Delivery room and majority of them within 10 minutes at first attempt. Thirty-two babies received Surfactant. Almost all babies (82) needed respiratory support in the form of either mechanical ventilation (41), CPAP/BiPAP (32) or High-flow Nasal cannula (9). All the pneumthoraces were confirmed with chest X-ray and/or transillumination except one needing emergency needle thoracocentesis in the delivery room. Respiratory distress was the main feature (38) followed by increasing respiratory support (28). Six pneumothoraces were identified following a routine chest $\mathrm{x}$-ray for line/tube placement. Pneumothoraces were commonly seen on right (40) and 18 neonates had bilateral pneumothoraces. Twenty-six resolved with expectant management while 53 needed either thoracotomy (30) or pigtail catheter (23). Three were drained by needle thoracocentesis. There was no significant difference in the complications seen with pigtail catheters or thoracotomy chest drains.

Conclusion

Common risk factors identified were preterm, respiratory distress, respiratory support.Commonest age at presentation $24 \mathrm{hrs}$. Conservative management successful in simple pneumothorax (34\%).No difference in the complications seen with pigtail catheters or thoracotomy - chest drains.

\section{6}

Neonatology

Clinical, infectious and biochemical characteristics of arterial hypotension in premature infants with early bacterial infections

Valeriy Pokhylko ${ }^{3}$, Tetiana Znamenska ${ }^{1}$, Tetiana Klimenko ${ }^{2}$, Olena Kovalova ${ }^{3}$, Yuliia Cherniavska

${ }^{1}$ Neonatology department, Institute of Pediatrics, Obstetrics and Gynecology of NAMS Ukraine, Ukraine

${ }^{2}$ Chair of Neonatology, Kharkiv medical academy of postgraduade education, Ukraine

${ }^{3}$ Chair of Pediatric \#1 with propedeutics and neonatology, Ukrainian medical stomatological academy, Ukraine

\section{Background}

Early onset infections play a big role in the number of adverse health effects among newborns, while their frequency is high enough. Namely 
hemodynamic disturbances often cause adverse events. Prediction of early neonatal sepsis in premature infants is imperfect, and pathogenetic mechanisms of hypotension syndrome and its correction - are not finally clarified. Objective

The aim of our study was to explore the clinical, infectious and metabolic manifestations of hypotension in premature infants and to justify diagnostic algorithm for determining hypotension syndrome and indications for use of hemodynamic support in premature infants with early bacterial infections.

Methods

Prospective observational study included 118 preterm infants with early neonatal sepsis $(n=57$ with arterial hypotension and $n=61$ without arterial hypotension) admitted to the neonatal intensive care units of children's hospitals of Poltava region.

\section{Results}

In premature infants with hypotension compared with children without hypotension, there are significantly lower average values of SILV (stroke index of left ventricle) $(p=0.005)$, IR ACM $(p=0.012), \mathrm{pH}(p=0.05)$, and significantly higher levels of urea $(p=0.002)$ in blood serum and more specific percentage of children with hypoglycemia $(p=0.013)$. Operating characteristics of predictive model that includes SILV, $\mathrm{pH}$, glucose

Conclusions

Our results were the basis for the definition of "hypotension syndrome", which includes the mean blood pressure of a child in mm less than gestational age in weeks and the presence of SILV less than the age limit, $\mathrm{pH}$ less than 7,2 , blood glucose less than $2.8 \mathrm{mmol} / \mathrm{l}$ and it can serve as a demonstration to the purpose of drug hemodynamic support, unlike the current situation where it is prescribed only under reduced pressure, which in premature infants may not be accompanied by adverse changes in organs and systems.

\section{4}

\section{Neonatology}

Use of Donor Expressed Breast Milk in Maternity Hospitals and Neonatal Units in the Republic of Ireland

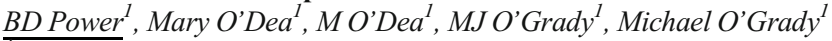
${ }^{T}$ Dept. of General Paediatrics, Regional Hospital Mullingar, Co. Westmeath, Ireland

\section{Background}

Over the past decade, where mothers own milk is unavailable, use of donor expressed breast milk (DEBM) has become increasingly common, particularly in VLBW infants. Practices and opinions regarding DEBM use in the Republic of Ireland (ROI) have not previously been established. Objective

To examine the current practices, opinions and knowledge regarding use of DEBM in Maternity Hospitals and Neonatal Units the ROI.

\section{Methods}

A standardized paper-based questionnaire was mailed to all Neonatologists and Paediatricians working in Maternity Hospitals and Neonatal Units in the ROI in 2016.

Results

Eighty-eight Paediatricians and Neonatologists were surveyed and 44 $(50 \%)$ responded. Responses were received from 21 neonatal units, of whom $15(71 \%)$ reported using DEBM. Fifty-three percent of units had a written DEBM policy, and significant variation existed in birthweight and gestational age thresholds for its use. Healthcare professionals were asked to indicate their level of agreement with a number of statements regarding DEBM. Twenty percent agreed that DEBM was more appropriate than formula use in healthy breastfed term neonates requiring supplementation and the same percentage agreed this practice increased long-term breastfeeding rates. One third of respondents agreed that the majority of trials supporting the use of DEBM to prevent necrotizing enterocolitis were conducted in the past 15 years, when the opposite is in fact the case. The main factors cited by physicians for not using DEBM in their unit were the specific population cared for in their unit, and inadequate access to DEBM.

\section{Conclusion}

Although donor milk is gaining considerable popularity, the criteria for its use are not well defined. This study identified wide variations in practice and deficits in knowledge regarding DEBM and indicates the need for a national guideline of evidence-based best practice for the use of DEBM for newborn infants in Ireland.

\section{8}

Neonatology

Shining a light on the icteric neonate; the use of transcutaneous bilirubinometers to reduce hospital admissions Irnthu Premadeva ${ }^{1}$, Shailee Sheth ${ }^{1}$, Kavita Chawla ${ }^{1}$ ${ }^{T}$ Paediatrics, Lister Hospital, UK

\section{Background}

A $£ 22$ billion efficiency requirement for the NHS makes cost-efficiency savings an important aspect of clinical practice in the UK. Short-term, less than () 1 day, hospital admission rates are increasing and, in the neonatal population, the commonest cause pertains to jaundice. Preventing unnecessary attendances with the use transcutaneous bilirubinometer $(\mathrm{TcB})$ in the community can ease the burden. National Institue of Clinical Excellence guidelines stipulate that $\mathrm{TcB}$ can be used in babies greater than () 35 weeks gestation at birth and 24 hours of life; serum bilirubin measurements are warranted if $\mathrm{TcB}$ reading is 250 micromoles/litre.

\section{Objective}

To identify whether the use of $\mathrm{TcB}$ readings can prevent hospital admission. Method

Data was collected on patients admitted to Children's Assessment Unit (CAU) with jaundice, between December 2016 and February 2017. Those who were older than 28 days and who did not have a TcB reading on arrival to the A\&E were excluded. Primary outcome was the proportion of babies with a $\mathrm{TcB}$ of $250 \mathrm{micromoles} / \mathrm{l}$. Secondary outcome measures were length of hospital stay for these patients and an assessment in the difference in weight loss between those requiring phototherapy and those who did not.

\section{Results}

In this cohort $(n=37)$ the average age at admission was 4.7 days; $72 \%$ $(n=27)$ had TcB 250micromoles/l. None of these patients required phototherapy during their admission, and the average length of stay was 5.5 hours. The average weight loss in patients requiring phototherapy $(n=7)$ and those who did not was $11 \%$, and $5 \%$ respectively.

\section{Conclusion}

The volume of patients attending CAU with jaundice is disproportionate to those who go on to require phototherapy and could have been prevented by the use of TcB readings in the community. The use of percentage weight loss as a marker of the severity of jaundice could further aid the community team in making a decision for or against hospital attendance.

\section{4}

Neonatology

Metabolomics in healthy neonates in Western Greece

Anastasia Varvarigou ${ }^{1}$, Ioannis Giannakopoulos ${ }^{1}$, Asimina Tsintoni ${ }^{1}$, Sotiris Fouzas ${ }^{1}$, Anastasios Koukouletsos ${ }^{4}$, Ioannis Koulouras ${ }^{4}$, Stavros Bariamis $^{2}$, Ioanna Georgakopoulou ${ }^{2}$, Stella Chasapi ${ }^{2}$, Manfred Spraul $^{3}$, Hartmut Schafer ${ }^{3}$, Georgios Spyroulias ${ }^{2}$

${ }^{1}$ Pediatrics and Neonatology, University Hospital of Patras, Greece

${ }^{2}$ Pharmacy, University of Patras, Greece

3Bruker Biospin, Bruker Biospin, Germany

${ }^{4}$ NICU, General Hospital of Patras, Greece

\section{Background}

Metabolomics represents a new and promising area of research in neonatology. To date, the method has been succesfully applied to monitor the rapid metabolic changes after birth and to detect the metabolic responses that may be characterestic for specific neonatal disorders. 


\section{Objectives}

The aim of our study was to develop a reference model of urinary metabolomics in healthy newborns up to their third day of life, taking also into account dietary effects, jaundice and exposure to tabacco smoke.

\section{Methods}

The study included 110 healthy newborns ( GA 35 to 40 weeks) from the region of Western Greece. Urine samples were collected immediately after birth and of the third day (DOL). Metabolic profiling of the samples was performed by H-NMR spectroscopy. Statistical analysis was conducted in R environment, using in-house scripts.

\section{Results}

Principal component analysis showed that there were significant diffferences from birth to DOL 3 in the relative intensities of the assigned metabolites, such as betaine, glycine and taurine. Trends in differentiation of metabolites levels between the two spactral groups, late preterm amd term newborns, were also observed.

\section{Conclusion}

Our preliminary data confirmed the rapid changes in the urinary metabolic profile after birth. Ongoing research will enable us to develop the reference model of urinary metabolomics in healthy newborns during the period of adaptation to the extra-uterine life.

\section{9}

\section{Neonatology}

Presence of Obstetric Risk Factors in a Late Preterm Newborn Group Compared to Full-Term Newborn

Alvaro J. M.O. Veiga ${ }^{l}$, Adriana Maria Tavares Gomes Gonçalves ${ }^{1}$, Pamela Andrade Montagni ${ }^{1}$, Mariana Dias Cipriano ${ }^{1}$, Lilian Terezinha Costa $^{I}$, Nathalia Veiga Moliterno ${ }^{I}$, Priscilla Magalhães Feleppa ${ }^{I}$, Eneida Quadrio Veiga ${ }^{I}$, Felipe Machado Moliterno ${ }^{I}$

${ }^{1}$ Neonatology, Faculty of Medicine of Petrópolis, Brazil

\section{Background}

Early neonatal sepsis is a systemic infection, presenting up to 72 hours after birth, usually associated with maternal risk factors, and remains an important cause of morbidity and mortality. It affects premature newborns (NB), the latter being the most vulnerable group, of which the late premature infants (born between 34 and 36 weeks and 6 days) represent about $70 \%$ of the total.

\section{Objective}

To identify the presence of obstetric risk factors for early neonatal sepsis in two different groups of neonates, full term and late preterm infants in a reference maternity hospital

Methods

Cross-sectional, descriptive study involving all newborns with gestational age above 34 weeks, born between February 1 and June 6, 2016 in the maternity hospital of the Alcides Carneiro Teaching Hospital. Data collected in structured form and previously tested.

\section{Results}

The incidence of late prematurity was $8.8 \%$, out of a total of 898 births. The presence of neonatal sepsis was higher in the group of preterm infants $(16.5 \%)$ than in term infants $(2.2 \%)$. The obstetric risk factors associated with early neonatal sepsis were: rupture of ovary membranes, preterm labor, chorioamnionitis, bag turnover time 18 hours, vaginosis. Regarding urinary tract infection during pregnancy, this study found $29.35 \%$ between the term NB and $5.96 \%$ at the time of delivery, whereas in the late PMT $40.5 \%$ and $11.39 \%$, respectively.

\section{Conclusions}

The mistaken similarity between late preterm newborn and full-term newborn leads us to the fact that they are approached without a differentiated look, which places them in conditions of marked risk and consequently increasing the incidence of morbidity and mortality. Thus, the increased attention to the prevention of severe diseases that these may present should be the focus of both pre and post natal care.
Obstetric risk factors for early-onset sepsis in late preterm and full term neonates

\begin{tabular}{l|c|c|c}
\hline & Late preterm & Term infants & \multirow{2}{*}{} \\
\cline { 2 - 3 } & $n=79(\%)$ & $n=819(\%)$ & \\
\hline Sepsis & $13(16,50)$ & $18(2,20)$ & $<0,05$ \\
\hline Prenatal care $(>6$ visits) & $54(67,11)$ & $680(82,78)$ & $<0,05$ \\
\hline Premature rupture of membranes & $25(31,60)$ & $56(6,84)$ & $<0,05$ \\
\hline Chorioamnionitis & $4(5,00)$ & $4(0,50)$ & $<0,05$ \\
\hline Vaginosis & $5(6,33)$ & $18(2,20)$ & $<0,05$ \\
\hline Stock exchange $>18$ hours & $19(24,10)$ & $49(6,00)$ & $<0,05$ \\
\hline Preterm labor & $42(53,20)$ & $5(0,60)$ & $<0,05$ \\
\hline $\begin{array}{l}\text { Urinary tract infection during } \\
\text { pregnancy }\end{array}$ & $32(40,50)$ & $241(29,43)$ & 0,05 \\
\hline Urinary tract infection at delivery & $9(11,40)$ & $49(6,00)$ & 0,087 \\
\hline
\end{tabular}

739

\section{Neonatology}

To identify the prevalence of live births with congenital malformations in a public maternity in the mountain region of the State of Rio de Janeiro - Brazil Alvaro J. M.O. Veiga ${ }^{l}$, Adriana Maria Tavares Gomes Gonçalves ${ }^{1}$, Nathalia Veiga Moliterno ${ }^{1}$, Lilian Terezinha Costa ${ }^{1}$, Maria Cristina Braga Soares ${ }^{1}$, Eneida Quadrio Veiga ${ }^{l}$, Felipe Machado Moliterno ${ }^{l}$, Lilian Terezinha Costa ${ }^{1}$

${ }^{I}$ Neonatology, Faculty of Medicine of Petrópolis, Brazil

\section{Background}

According to the World Health Organization, congenital anomaly or congenital defects, congenital diseases or congenital malformations is defined as any functional or structural anomaly in the development of the fetus, due to socioeconomic factors, genetic factors, infections caused by nutritional, maternal and Environmental impacts. About 2 to $5 \%$ of newborns in Brazil and around the world have some type of congenital anomaly, which can be classified as major or minor.

\section{Objective}

To identify the prevalence of congenital malformations in neonates in a public maternity.

\section{Methods}

Cross-sectional, descriptive study involving all births occurred in the public maternity of the Alcides Carneiro Petrópolis-RJ-Brazil Teaching Hospital, from January 2 to June 6, 2016. Data were obtained from the charts of the newborns and their mothers, upon the birth of the newborn. Results

A total of 900 neonates were studied in the study period, with a prevalence of congenital anomalies in 33 newborns, or $3.8 \%$, classified in the majority of cases in isolation, and of less clinical importance, and it was not necessary to transfer them to the care unit Intensive. There was a prevalence in the group with male malformations $66.7 \%$, female $33.3 \%$. In relation to gestational age, there was a prevalence of congenital anomalies among children between 34 and 36 weeks of age in relation to the group of full-term newborns (p 0.05). Although not statistically significant, the birth weight in the group of congenital anomalies was $3045 \pm 538 \mathrm{~g}$ versus $3219 \pm 870 \mathrm{~g}(p=0.07)$.

\section{Conclusion}

The prevalence of congenital anomalies in our study is in line with findings in the literature and has been more prevalent in a group of preterm 
infants. Knowing the prevalence of congenital anomalies can serve as an instrument for health planning, ensuring access and quality of care for women and newborns.

\begin{tabular}{|c|c|c|}
\hline \multicolumn{3}{|c|}{$\begin{array}{l}\text { Table I: Classification of the types of congenital } \\
\text { malformation }\end{array}$} \\
\hline Malformation & $\mathrm{N}$ & $\%$ \\
\hline Osteomuscular & 11 & $33,33 \%$ \\
\hline Genital organs & 10 & $30,30 \%$ \\
\hline Eye, ear, face and neck & 6 & $18,18 \%$ \\
\hline Central Nervous System & 3 & $9,09 \%$ \\
\hline Circulatory System & 2 & $6,06 \%$ \\
\hline Chromosomal anomaly & 1 & $3,03 \%$ \\
\hline Total & 33 & $100 \%$ \\
\hline
\end{tabular}

746

Neonatology

Neonatal outcomes of women with pre-gestational excess weight Rashmi Ranatunga ${ }^{l}$, Kapila Jayaratne ${ }^{1}$

${ }^{T}$ Maternal and Child Morbidity and Mortality Surveillance Unit, Family Health Bureau - Ministry of Health, Sri Lanka

\section{Background}

An increasing trend of prevalence of overweight and obesity is noted worldwide over the past few decades. Pre-gestational excess weight has shown negative impact on neonatal outcome. Solid data are essential in formulating preventive strategies to break the viscous cycle of excess weight in pregnancy.

\section{Objectives}

To describe neonatal outcomes of women with pre-gestational excess weight delivered at a tertiary care hospital in Sri Lanka, a South Asian country.

To describe the association between maternal pre-gestational body mass index (BMI) with neonatal macrosomia and their abdominal circumference.

\section{Methods}

A hospital-based descriptive cross sectional study was conducted. A sample of 535 women with reported pre-gestational BMI $25 \mathrm{~kg} / \mathrm{m} 2$ in antenatal pregnancy record delivering at postnatal wards of Colombo North Teaching Hospital were recruited using non-probability consecutive sampling method. An interviewer-administered questionnaire was used for data collection.

A sample of 155 macrsomic ( $3.5 \mathrm{Kg}$ ) babies were recruited from the same ward settings and compared with 155 normal weight babies in a casecontrol analysis to ascertain maternal obesity with macrosomia.

\section{Results}

Majority of newborns were male $(n=307,57.4 \%)$ compared to female babies $(n=228,42.6 \%)$. A significant proportion $(n=85,15.9 \%)$ of babies were delivered premature. Birth weight was within $2.5 \mathrm{Kg}-3.49$ $\mathrm{Kg}$ in $55.1 \%(n=101)$, macrosomic $26 \%(n=139)$ and low birth weight $18.9 \%(n=295)$. Many $68.7 \%(n=307)$ reported neonatal morbidity and $57.4 \%(n=307)$ babies needed admission to Special Care Baby Unit. Five $(1.1 \%)$ still births and 1 neonatal deaths were reported.

Increased maternal BMI is at high risk of macrosomic baby $(\mathrm{OR}=110, \mathrm{CI}$ 49.21-246.47) and neonatal abdominal circumference $30 \mathrm{~cm}(\mathrm{OR}=11.8$, CI 5.58-24.94).

\section{Conclusions}

Pre-gestational maternal excess weight is associated with multiple neonatal adverse outcomes; premature delivery, macrosomia, low birth weight, neonatal complications and high abdominal circumference. These can be prevented by pre-pregnancy counselling and planned pregnancies with appropriate BMI.

820

Neonatology

Comparison of Laryngeal Mask Airway and Endotracheal Tube Placement in Neonates

Amanda Wanous ${ }^{1}$, Kyle Rudser ${ }^{2}$, Kari Roberts ${ }^{1}$

${ }^{I}$ Pediatrics, University of Minnesota, USA

${ }^{2}$ Biostatistics, University of Minnesota, USA

\section{Background}

With increased use of non-invasive ventilation, there has been an increased focus of research on less invasive methods of delivering surfactant to neonates with respiratory distress syndrome. We investigated use of a laryngeal mask airway (LMA) for surfactant administration for infants requiring continuous positive airway pressure. Infants who met treatment failure criteria in the LMA and Control groups were intubated and given surfactant via an endotracheal tube (ETT).

Objective

This study compares placement of an LMA to placement of an ETT in neonates.

\section{Methods}

This is one component of a multicenter, randomized controlled trial. Infants were 28 0/7- 35 6/7 weeks gestation, $\geq 1250$ grams and $\leq 36$ hours old. Videotape of LMA $(n=36)$ and ETT $(n=31)$ placement were reviewed to determine the time and number of attempts required for successful placement. Heart rate (HR) and oxygen saturation $\left(\mathrm{SaO}_{2}\right)$ change from baseline were analyzed as measures of physiologic stability during placement.

\section{Results}

Duration of attempts was shorter for LMA as compared to ETT placement (32 sec vs $66 \mathrm{sec}, \mathrm{p} 0.001)$. Mean total procedure time for successful LMA placement was $88 \mathrm{sec}$ as compared to $153 \mathrm{sec}$ for ETT $(p=0.065)$. Mean number of attempts for successful placement was fewer for LMA placement (1.5 vs 1.9, p0.106). Physiologic parameters remained near baseline in both LMA and ETT groups with HR change $+1 \mathrm{bpm}$ and $-1 \mathrm{bpm}(p=0.333)$ and $\mathrm{SaO}_{2}$ change $-7 \%$ and $-4 \%$ $(p=0.361)$, respectively.

Conclusions

Placement of an LMA was well tolerated and required less time and fewer number of attempts as compared to the traditional method of endotracheal intubation.

\section{2}

\section{Neonatology}

Cesarean section strongly impacts stool microbiota of Bangladeshi infants in low-income settings

Olga Sakwinska ${ }^{I}$, Harald Brüssow ${ }^{I}$, Bernard Berger ${ }^{I}$, Francis Foata ${ }^{I}$, Nadine Porta $^{1}$, Aline Charpagne ${ }^{2}$, Zeenat Arefin ${ }^{3}$, Iztiba Mallik ${ }^{3}$, Patrick Descombes ${ }^{2}$, Shamima Sultana ${ }^{3}$, Shafiqul Alam Sarker ${ }^{3}$

${ }^{1}$ Nestlé Research Center, Nestec, Switzerland

${ }^{2}$ Nestlé Institute of Health Sciences, NHSc, Switzerland

${ }^{3}$ Nutrition and Clinical Services Division, International Centre for Diarrheal Diseases Research, Bangladesh

The delivery by Cesarean section (CS) is becoming increasingly common, including in middle-income settings. CS has been linked to later in life (infancy and childhood) health problems, including increased susceptibility to infections and chronic immune 
disorders. Infant gut microbiota has been hypothesized to play a key role in these outcomes.

We set out to examine the impact of delivery mode on initial stool microbiota of infants from periurban community in Bangladesh, Dhaka. The delivery mode was decided jointly by the family and the obstetrician independently of the participation in the study. Stool samples were collected from 263 infants between the day of birth and day 3. The composition of stool microbiota was evaluated by $16 \mathrm{~S}$ rRNA gene sequencing.

67 out of 263 infants (26\%) were delivered by CS. The CS rate was similar among primiparous women $(30 / 107,28 \%)$, and women who had already given birth before $(37 / 156$, $24 \%)$. Notably, only 4 out of 156 women (2.6\%) delivered by $\mathrm{CS}$ in their previous pregnancy (delivery on average 5 years before).

CS had a clear impact on stool microbiota. The stool microbiota of CS delivered infants was dominated by Klebsiella $(37 \%)$ and other Enterobacteriaceae (17\%) while vaginally delivered (VD) infants harbored mostly Escherichia (58 \%) and less Klebsiella $(10 \%)$. In VD infants, on the day of birth, the relative abundance of Escherichia was $32 \%$ and increased to $78 \%$ on day 3. In CS delivered infants, Escherichia was absent until day 1, and reached $23 \%$ only on day 3 .

We have observed a ten-fold increase in CS rate from $2.6 \%$ to $24 \%$ over a time span of 5 years in a periurban community in Dhaka, Bangladesh. The usual process of gut colonization appears to be delayed after CS. To better understand the long-term impact of the delivery mode on infant microbiota and associated infant health outcomes, the health of infants in this cohort will be monitored for the first 2 years of life.

279

Neonatology

Protecting the Next Generation from Superbugs: Is there a Role for Decontamination? Experience from a Major Neonatal Centre in the UK

Camilla Sen ${ }^{l}$, Alexandra Lemaigre ${ }^{l}$, Tulsi Patel ${ }^{l}$, Chloe Norman ${ }^{l}$, Helen Egan $^{I}$, Gregory Landon ${ }^{1}$, Shu-Ling Chuang ${ }^{I}$, Enitan Ogundipe ${ }^{I}$

${ }^{1}$ Neonatal, Chelsea and Westminster Hospital, UK

\section{Background}

Increasing antimicrobial resistance is a global problem causing significant morbidity and mortality in neonates.

\section{Objective}

We aimed to assess whether increasing antibiotic resistance (AR) was a major issue in our neonatal unit.

Hypotheses

- Bacteria causing colonization may later cause invasive infection. If so, decontamination of a colonized patient may prevent sepsis.

- Central-line penetration of the skin barrier will increase sepsis particularly with MSSA.

Methods

We analysed all positive blood cultures (BC) 01/01/2011 - 31/ $12 / 2015$ from our neonatal unit. We eliminated contaminated $\mathrm{BC}$ based on inflammatory markers and reference to clinical notes. We collected data on BC species, sensitivities, patient colonization, comorbidities, central-line access and antibiotic treatment.

\section{Results}

Four hundred ninety-six positive BC were taken between 01/01/ $2011-31 / 12 / 2015$. We excluded 36 due to normal inflammatory markers and clinical suspicion of contamination.

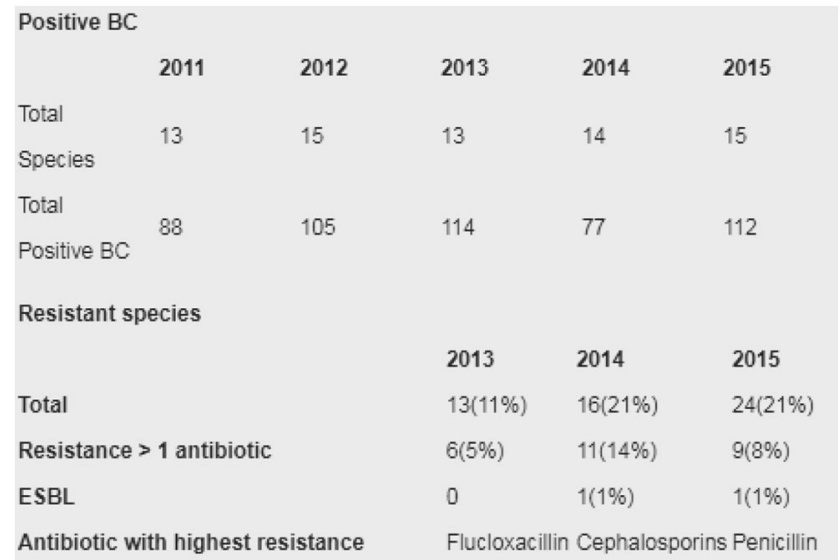

\begin{tabular}{|c|c|c|}
\hline \multicolumn{3}{|l|}{ Patients with positive $B C$} \\
\hline 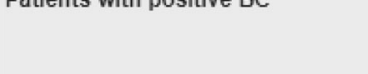 & 2014 & 2015 \\
\hline$<37 / 40$ gestation & $62(80 \%)$ & $94(84 \%)$ \\
\hline Necrotising enterocolitis & $31(40 \%)$ & $45(40 \%)$ \\
\hline Other surgical diagnosis & $12(16 \%)$ & $13(12 \%)$ \\
\hline Central line at time of $\mathrm{BC} /$ removed & $50(65 \%)$ & $60(54 \%)$ \\
\hline $\begin{array}{l}\text { Previous swab with same bacteria } \\
\text { (colonization) }\end{array}$ & $6(8 \%)$ & $24(21 \%)$ \\
\hline MSSA +BC with MSSA colonization & 0 & 0 \\
\hline Death related to sepsis & 0 & $5(4 \%)$ \\
\hline
\end{tabular}

\section{Conclusion}

Antibiotic resistance (AR) is a worsening problem affecting $11 \%$ of BC in 2013 and $21 \%$ in 2014-2015. Sixteen percent of sepsis episodes (2014-15) were caused by bacteria which colonized the patient but none caused by MSSA. Therefore, decontamination may be of benefit but this should be weighed against the risk of increasing AR. Consider decontamination when there are additional risks for sepsis e.g. central lines.

Future research should trial decontamination when multiple risk factors for sepsis are present to determine the risk of increased resistance versus sepsis reduction.

\section{7}

\section{Neonatology}

Change of Gentamicin Dose in Newborns with Early Onset Sepsis: Audit and Quality Improvement project

Francesca Seregni ${ }^{I}$, Heather Crowley ${ }^{I}$, Christine Naganathan ${ }^{I}$, Sin-Hui Toh ${ }^{I}$, Sanjay Raina

${ }^{I}$ Neonatal Intensive Care Unit, Princess Alexandra Hospital, UK

Gentamicin is an aminoglycoside antibiotic, widely used as first line in the treatment of confirmed or suspected neonatal sepsis because of its good cover against gram negative bacteria. Gentamicin is known for its side effects, which include nephrotoxicity (1.6-10\%) and ototoxicity (2$2.3 \%$ ), and for this reason strict monitoring of trough level is mandated. We suspected that our unit was frequently recording high gentamicin levels when administering the drug at $5 \mathrm{mg} / \mathrm{kg}$ 24-hourly in more than 32 weeks babies, and 36-hourly in less than 32 weeks (Dosage 1). 
We audited 100 babies treated with Dosage 1 and found that $35 \%$ of these developed toxic gentamicin levels $(2 \mathrm{mg} / \mathrm{L}$ pre second dose or $1 \mathrm{mg} / \mathrm{L}$ pre fifth dose). Overall, toxicity was higher in preterm than term babies $(p=0.018)$. In particular, $54 \%$ of preterm babies born between 32 and 38 weeks had a toxic level. We also noticed that only $62.85 \%$ of babies with high levels were appropriately referred to audiology for extended hearing test at 6-8 months of life.

As a consequence, we adopted in our Trust new regional antibiotic guidelines, which suggested a different dosing for gentamicin: $5 \mathrm{mg} / \mathrm{kg} \mathrm{36-}$ hourly for the first 7 days of life irrespective of the gestational age (Dosage 2). We also changed the referral pathway to audiology.

We then re-audited gentamicin levels in 100 newborns treated with Dosage 2. The results show that toxicity levels fell from $35 \%$ to $3 \%$ (p0.001) with Dosage 2. Three patients still showed above reference trough levels: 1 had suffered from a hypoxic insult at birth and the remaining 2 babies had been admitted to NICU for hypoglycaemia and severe sepsis. One hundred percent of babies with high levels were appropriately referred to audiology as per the new pathway.

In conclusion, with Dosage 2 we were able to significantly reduce the number of recorded gentamicin toxic level.

\section{6}

\section{Neonatology}

Daily supplementation with 400 IU Vitamin D in Term Breast Fed Infants from 0-6 Months and Changes in Total and Bone Specific Alkaline Phosphatase-A RCT

Hemlata Singh ${ }^{1}$, Amlin Shukla ${ }^{1}$, Arti Maria ${ }^{1}$, Kalaivani Mani ${ }^{2}$

${ }^{I}$ Department of Neonatology, PGIMER \& Dr. RML Hospital, India

${ }^{2}$ Department of Biostatistics, AIIMS, India

\section{Background}

Vitamin D deficiency amongst pregnant women and their newborns is prevalent in India. Total Alkaline Phosphatase(TALP) and Bone Specific Alkaline Phosphatase(BSALP)are surrogate markers of vitamin D activity. Although recommendations exist for supplementation of exclusively breast fed infants with vitamin D but there is a paucity of data and lack of recommendations from our country regarding vitamin D supplementation and dosage.

Objectives

Primary: To study the effect of daily supplementation with oral 400 IU vitamin D on changes in TALP and BSALP in healthy term exclusively breast fed infants(intervention group) from birth until 6 months as compared to non-supplemented (control) group.

Secondary: To compare change in vitamin D, PTH, calcium, phosphorus levels between the study group and control group.

Method

It was an open label RCT in postnatal ward setting of a tertiary care centre in North India. One hundred term healthy exclusively breast fed babies were randomised into two groups by block randomisation and allocation concealment either to receive or not to receive vitamin D. Baseline levels of studied parameters were measured from cord blood. Both groups were followed for 6 months, investigations were repeated at the end of this period and changes in studied parameters in both groups were compared. Results

Ninety-one percent mothers and $60 \%$ babies were vitamin D deficient $(20 \mathrm{ng} / \mathrm{ml})$ at birth. At 6 months $40 \%$ babies had vitamin D deficiency in supplemented group, significantly less than $76 \%$ in control group $(\mathrm{p}$ $0.05)$. There was significant difference in change in levels of $\operatorname{TALP}(\mathrm{p} 0.05)$ but not in $\operatorname{BSALP}(p=0.14)$ between two groups at 6 months. Conclusions

Vitamin D supplementation (400 IU/day) in healthy term newborns from birth significantly reduces vitamin D deficiency at 6 months of age and leads to significant changes in TALP but response of BSALP probably needs to be studied for a longer duration and over a larger group to predict effect on bone mineralisation.
489

\section{Neonatology}

What about genetic counseling in prenatally detected supernumerary chromosome marker cases

Wafa Slimani ${ }^{1}$, Molka Kammoun ${ }^{1}$, Saoussen Meddeb ${ }^{2}$, Mouna Kouira ${ }^{4}$, Amani Hajjaji ${ }^{3}$, Anouar Chaieb ${ }^{4}$, Thomas Liehr ${ }^{5}$, Ali Saad ${ }^{1}$, Soumaya Mougou-Zerelli ${ }^{l}$

${ }^{T}$ Human Cytogenetics, Laboratory of Human cytogenetics, Molecular Genetics and Biology of Reproduction, Farhat Hached University Hospital, Tunisia

${ }^{2}$ Department of Gynecology and obstetrics, Farhat Hached University Hospital, Tunisia

${ }^{3}$ Department of Gynecology and obstetrics, Fattouma Bourguiba hospital, Tunisia

${ }^{4}$ Unit of Reproductive Medicine, Farhat Hached University Hospital, Tunisia

${ }^{5}$ Human Genetics, Institute of Human Genetics, Germany

\section{Background}

Supernumerary chromosome markers (SMC) are rare chromosomal abnormalities that are difficult to identify by conventional cytogenetic techniques alone. They are found in $0.044 \%$ of live births and are seven times more frequent in cases of intellectual disability. Their prenatal prevalence is $0.075 \%$. This frequency appears to be reduced because 30 to $50 \%$ of pregnancies with a fetus carrying SMC are interrupted. Diagnosis of $\mathrm{SMC}$ is always challenging especially when detected prenatally.

\section{Objective}

The aim of this study was to characterize prenatally detected SMC in five pregnant women and to offer a genetic counseling to the parents.

\section{Methods}

Amniocentesis, R-Banding karyotype and Fluorescent in Situ Hybridization (FISH) were performed in five patients referred to our department at 17 weeks of gestation for advanced maternal age and/or a history of trisomy 21 or abnormalities in ultrasound evaluation.

\section{Results}

Amniocentesis showed a small SMC present homogeneously for two patients, mosaic and de NOVO in three. FISH was performed on amniotic cells using specific probes for 15, 22, 20 and 18 chromosomes. The SMC was found to be a derivative 20 in two fetuses, 1 derivative from heterochromatic regions of 13/ 21 chromosomes and derivative 22 in the latter. Follow-up has been proposed and postnatal Karyotypes were performed in 3 patients. Der 13/21 was inherited from a healthy mother. Interestingly, for the derivative 22, postnatal karyotypes on both lymphocytes and fibroblasts was normal. Likewise, karyotype on lymphocytes was normal for one of the derivatives 20 .

\section{Conclusion}

Genetic counseling was given to parents who decided to continue the pregnancy, which had normal outcome in all cases. Discordant results from amniocentesis and postnatal karyotypes suggest the existence of confined placental mosaicism. Our study confirms that amniotic fluid cells alone are not sufficient to predict mosaic SMC's outcome.

\section{7}

\section{Neonatology}

Supernumerary Marker Chromosomes encompassing the 15q11q13 region: about 6 new Tunisian cases

Wafa Slimani ${ }^{I}$, Molka Kammoun ${ }^{1}$, Ahlem Atig ${ }^{1}$, Saoussen Meddeb ${ }^{2}$, Jihen Mathlouthi ${ }^{3}$, Thomas Liehr ${ }^{4}$, Ali Saad ${ }^{1}$, Soumaya Mougou-Zerelli ${ }^{1}$ ${ }^{1}$ Human Cytogenetics, Laboratory of Human Cytogenetics, Molecular Genetics and Biology of Reproduction, Farhat Hachad University Teaching Hospital, Tunisia

${ }^{2}$ Department of neonatology, Department of neonatology, CHU Farhat Hached de Sousse, Tunisia

${ }^{3}$ Pediatric Department, Pediatric Department, CHU Farhat Hached de Sousse, Tunisia

${ }^{4}$ Human Genetics, Institute of Human Genetics, Germany 


\section{Background}

Supernumerary marker chromosomes (SMC) are additional abnormal chromosomes that can be derived from any chromosome. However, more than $50 \%$ of SMC are derived from acrocentric chromosomes, mainly chromosome15.

\section{Objective}

The aim of this study was to characterize six SMC detected in 6 children presenting heterogeneous phenotype ranging from mild to severe intellectual disability and/or developmental delay and dysmorphic features. Autism spectrum disorder, seizure and speech delay were noted in two patients.

\section{Methods}

R-Banding karyotypes of patients and their parents showed a de Novo SMC 15 in all cases except for one where the marker was maternally inherited, and one was in mosaic. In situ fluorescence hybridization (FISH) analysis using the SNRPN, GABRB3, IGF1R, Midi54, Sc15q, SE15 and WCP15 probes allowed us to determine the shape of these SMC and to delimit approximately the breakpoints.

\section{Results}

In three cases, the SMC was of inverted duplicated shape, encompassing the Prader-Willi/Angelman critical region (PW/ASCR). In two cases, the SMC had smaller size and did not contain the PW/ASCR. A disproportional subset of SMC is derived from chromosome 15 conferring a trisomy or tetrasomy of the $15 \mathrm{q} 11-\mathrm{q} 13$ region. In fact, this region is prone to unequal crossover responsible for the variable size of chromosomal rearrangements due to the existence of five known breakpoints. It is also a parentally imprinted region that undergoes differential phenotypic expression depending on the parental origin.

\section{Conclusion}

The presence of breakpoints, parental imprinting region and $\mathrm{PW} /$ ASCR, the content of euchromatic material and mosaicism are responsible for the morphological and clinical heterogeneity of the SMC15. The study of the extent of the duplicated region by array CGH would allow to identify the exact segmental gains and to delimit precisely the breakpoints.

568

\section{Neonatology}

Birth size of infants born near-term after In-Vitro Fertilization (IVF) Compared to Normal Infants

Ahmed F Eldakrouri ${ }^{I}$, Hamdy A Alsayed ${ }^{1}$, Hilal AlRifai ${ }^{I}$, Ashraf Soliman $^{2}$

${ }^{1}$ Neonatology, Hamad Medical Center, Qatar

${ }^{2}$ Pediatrics, University of Alexandria, Egypt

\section{Objective}

Due to recent scientific progress in assisted reproductive techniques (ART), infertile couples can now become fertile. Thus, a number of infants in our country are the results of these costly interventions. This study has been undertaken to evaluate the physical growth process of different methods of ART infants by standard growth charts from birth until nine months of age.

\section{Methods}

We measured the anthropometric data of 100 infants newborns conceived through ART [intracytoplasmic sperm injection (ICSI) at HMC. A sequential sampling method in a period of 2 years was used. Their birth size was assessed by measuring infants' weight, height and head circumference, and physical examination. The weight for age SDS (WSDS), length SDS (LSDS) and head circumference SDS (HCSDS) were calculated with reference to normal standard for gestational age and sex of the newborn.

\section{Findings}

In comparison with normal growth standards for gestational age and gender, the HCSDS was 2SDS in 3/100 infants, the LSDS was -2 in $6 / 100$ infants, and WSDS was -2 in $16 \%$ of the infants. Low birth weight (LBW) infants were twice more in the ART group compared to infants of normal population.

\section{Conclusion}

Infants born with ART have normal intrauterine growth appropriate for their gestation age. However, they are more susceptible to be underweight at birth than normal infants.

\begin{tabular}{llll}
\multicolumn{4}{c}{ Gestation age $(W) W t$ SDSLSDSHC SDS } \\
Mean 36.40 & -0.85 & -0.23 & -0.36 \\
SDS 1.70 & 0.98 & 0.91 & 0.77
\end{tabular}

406

Neonatology

Effects of perinatal factors on body mass index and exercise capacity of school-age children

Irena Štucin Gantar ${ }^{1}$, Miha Lučovnik ${ }^{1}$, Gregor Starc ${ }^{2}$, Petra Golja ${ }^{3}$, Lilijana Kornhauser Cerar ${ }^{1}$

${ }^{1}$ Clinic for Gynecology and Obstetrics, University Medical Centre Ljubljana, Slovenia

${ }^{2}$ Faculty of Sport Ljubljana, University of Ljubljana, Slovenia

${ }^{3}$ Biotechnical Faculty Ljubljana, University of Ljubljana, Slovenia

\section{Objective}

To identify the possible long term effects of perinatal factors on child's body mass index (BMI) and fitness capacity.

\section{Methods}

Data from two registries, the SLOFIT database and NPIS, were linked to evaluate the potential effects of perinatal factors on children's body mass index (BMI) and exercise capacity (PFI). We analyzed SLOFIT data of 2929 children from 2016 and linked them to data of 6894 born infants ( 90 stillbirths and neonatal deaths excluded) at our institution in 2008. Our subjects were thus 8 (SD) years old on average. Linear regression analysis was used to assess the potential relationship between child's BMI or child's physical fitness index (PFI) and perinatal factors.

\section{Results}

We identified 2929 (43\%) of children in the SLOFIT database born in our institution in 2008. Child's BMI at school-age was positively associated with maternal pre-pregnancy BMI (p0.001), and inversely associated with term birth $(p=0.02)$ and mother's education level ( $\mathrm{p} 0.001)$. Child's PFI at school-age was positively associated with school grade (p0.001), birth weight $(p=0.006)$, and maternal education ( $\mathrm{p} 0.001)$. PFI was inversely associated with nuliparity (p0.001), lower maternal education $(p=0.003)$, maternal pre-pregnancy BMI (p0.001), and Neonatal Intensive Care Unit (NICU) admission $(p=0.020)$.

Conlusions

Maternal pre-pregnancy BMI and education level are associated with BMI and fitness capacity at the age of 8 years. Firstborns and those admitted to NICU had lower fitness capacity. Being born very preterm had no significant impact neither on BMI or PFI.

\section{1}

\section{Neonatology}

Failure of Humidified High-Flow Nasal Cannulae: A Retrospective Analysis Sophia Teoh ${ }^{1}$, Elizabeth Clyde ${ }^{I}$, Theodore Dassios ${ }^{I}$

${ }^{T}$ Neonatal Unit, King's College Hospital, UK

\section{Background}

The use of Humidified High-Flow Nasal Cannulae (HHFNC) as an alternative mode of non-invasive ventilation in neonates has become widespread in neonatal practice. Identification of factors contributing to the failure of HHFNC is therefore important. 


\section{Objective}

To report clinical and demographic characteristics of the infants that fail treatment with HHFNC.

\section{Methods}

The records of all infants admitted to the neonatal unit of King's College Hospital (London, UK) over 2 years from 01/01/2015 to 31/12/2016 who went onto HHFNC were reviewed. Information was taken from hospital notes and the electronic patient data system BadgerNet. Comparison was made between the babies who failed on HHFNC and those who successfully weaned off HHFNC. Failure was defined as the need to switch to continuous positive airway pressure (CPAP) or intubation and mechanical ventilation. Success was defined as weaning off all respiratory support or to supplemental oxygen delivered by low flow nasal cannulae. In cases where the babies had multiple episodes of treatment with HHFNC, only the first episode was reviewed.

\section{Results}

Two hundred and twenty-six infants were identified; data were obtained for 154. Of these, 134 were suitable for use. Infants that failed HHFNC had a lower mean birthweight compared to those who succeeded (1376 g versus $1603 \mathrm{~g}$ respectively, $p=0.035$ ), higher mean $\mathrm{FiO}_{2}$ when starting HHFNC ( 0.34 vs 0.28 respectively, $p=0.004$ ), and more frequently had a positive blood culture at the time of starting HHFNC ( $9 \%$ vs $2 \%$ respectively, $p=0.001$ ). Multivariate regression analysis of birth weight, blood culture and $\mathrm{FiO}_{2}$, using failure of $\mathrm{HHFNC}$ as the outcome, revealed that the mean $\mathrm{FiO}_{2}$ at the time of starting HHFNC was statistically significant (p0.05), whilst the birthweight and the incidence of positive blood culture were not. Conclusion

Failure of HHFNC is more common among infants with a higher oxygen requirement at the point when HHFNC is started.

\section{1}

\section{Neonatology}

Effects of Ventilatory Settings on Oropharyngeal Gas Conditioning in a Bench Model of Nasal High-Frequency Oscillatory Ventilation $\underline{\text { Tim Ullrich }}^{1}$, Christoph Czernik ${ }^{1}$, Christoph Bührer ${ }^{I}$, Gerd Schmalisch ${ }^{1}$, Hendrik Fischer ${ }^{1}$

${ }^{1}$ Klinik für Neonatologie, Charité Universitätsmedizin Berlin, Germany

\section{Background}

Nasal high-frequency oscillatory ventilation (nHFOV) is a novel mode of non-invasive ventilation used in neonates. However, upper airway obstructions due to viscous secretions have been described as characteristic side effects of nHFOV. Hypothetically, high frequency oscillations may impair oropharyngeal gas conditioning and cause upper airway dryout.

\section{Objective}

To investigate the effects of nHFOV ventilatory settings on oropharyngeal temperature $(\mathrm{T})$ and humidity in a neonatal bench model.

\section{Methods}

Nasal continuous positive airway pressure (nCPAP) or nHFOV were applied by a neonatal ventilator (Leoni Plus, Heinen \& Löwenstein) at a mean pressure of $8 \mathrm{cmH}_{2} \mathrm{O}$ in conjunction with a heated humidifier (MR850, Fisher \& Paykel). T and humidity were measured by a thermohygrometer inside a model oropharynx using a previously established bench model. In a first set of measurements, nCPAP and different nHFOV frequencies $(7,10,13 \mathrm{~Hz})$ and amplitudes $\left(10,20,30 \mathrm{cmH}_{2} \mathrm{O}\right)$ were tested. In a second set, different inspiratory to expiratory (I:E) ratios $(25: 75,33: 66$ and 50:50) were tested at fixed amplitude and frequency. Measurements were repeated ten times for each parameter setting.

\section{Results}

The breathing gas in the model oropharynx was always fully saturated (relative humidity $99.9 \%$ ). NHFOV resulted in lower mean T and absolute humidity $(\mathrm{AH})$ in comparison to nCPAP $\left(\mathrm{T} 33.8 \pm 0.7{ }^{\circ} \mathrm{C}, \mathrm{AH}\right.$ $37.3 \pm 1.4 \mathrm{~g} \cdot \mathrm{m}^{-3}$ vs T $34.8 \pm 0.6{ }^{\circ} \mathrm{C}$, AH $\left.39.3 \pm 1.3 \mathrm{~g} \cdot \mathrm{m}^{-3}, \mathrm{p} 0.001\right)$. Decreasing nHFOV frequency and increasing amplitude resulted in an additional decline of $\mathrm{T}$ and $\mathrm{AH}$ (p0.001, respectively). For frequencies over $10 \mathrm{~Hz}$, the effect was no longer dependent on frequency, but on amplitude alone $(p=0.022)$. Increasing the I:E ratio led to a further decrease in T and AH ( $p=0.03$, respectively).

\section{Conclusion}

NHFOV resulted in impaired oropharyngeal gas conditions in comparison with nCPAP. Intensification of nHFOV frequency, amplitude and I:E settings caused further deterioration. Future studies should explore strategies to improve heated humidification during nHFOV.

\section{0}

\section{Neonatology}

Introducing the "Druggle": Quality Improvement Project to Improve Prescribing in a Tertiary Neonatal Unit

Hannah Vawda ${ }^{l}$, Preeya Rehsi ${ }^{1}$, Gemma Holder ${ }^{1}$

${ }^{I}$ Neonatal Unit, Birmingham Women's and Children's Hospital NHS Foundation Trust, UK

\section{Background \& Objective}

A consistently high number of prescription errors identified via our Datix system in our tertiary neonatal unit (NNU) led to medication errors being listed as a red risk on our Risk Register. The weekly "Druggle" was implemented to improve the standards of prescribing and medication administration.

\section{Methods}

Prescription charts were reviewed at random on a weekly basis to assess adherence to local and national prescribing standards. The five minute 'Druggle' was presented weekly during the morning multidisciplinary handover huddle. This highlighted examples of good and poor prescriptions anonymously to identify common pitfalls. Learning points were shared and a 'Prescriber of the Week' was identified. Following it's introduction, prescriptions were assessed weekly to determine whether the 'Druggle' resulted in improvements in prescribing practice.

\section{Results}

Qualitative and quantitative feedback showed improvement within certain elements of prescribing on a week-to-week basis as the audit period progressed, suggesting a positive impact following introduction of the "Druggle".

\section{Conclusion}

Through introduction of the "Druggle" we have identified a successful rewards based process to improve prescribing standards in the neonatal unit and thereby improve patient safety and reduce medication errors. We propose to continue this process on a 6 monthly basis through regular election of auditors/prescribing champions to continue to improve prescribing practice on the NNU.

\section{8}

\section{Neonatology}

Establishment of the Vascular Injury Model of Retinopathy of Prematurity in vitro Ruyuan Zhu ${ }^{1}$, Huijuan $\mathrm{Li}^{1}{ }^{1}$ Li Jiang

Pediatrics, Zhongda Hospital Affiliated to Southeast University, Nanjing, China

\section{Background}

The early stage of retinopathy of prematurity (ROP) mainly presented as retinal vascular retardation. The most important causes of ROP are hyperoxia/hypoxia injury, inflammation, etc. ROP rat model, namely the oxygen-induced retinopathy (OIR) model, was conducted by intermittent hyperoxia and hypoxia stimulation for 14 days in neonatal rats. During 0-14 days, retinal vasculature appeared dysplasia, and failed to reach the edge of the retina. Although the animal model is mature, the cell model which is 
consistent with the vascular endothelial cell injury of ROP is lacked.

\section{Objective}

This study was designed to explore the in vitro model of retinopathy of prematurity.

\section{Methods}

The experiment group was treated with hyperoxia-induced complete medium (containing $50 \mu \mathrm{mol} / \mathrm{L} \mathrm{H}_{2} \mathrm{O}_{2}$ solution) for $15 \mathrm{mi}$ nutes and hypoxia-induced complete medium (containing $50 \mu \mathrm{mol} / \mathrm{L} \mathrm{CoCl}_{2}$ solution) for 4 hours. Two cycles of hyperoxia/hypoxia stimulation were conducted. Wound healing kinetic and transwell migration assay were used to test the proliferation and migration abilities of the HUVEC.

\section{Results}

After two cycles of stimulation, cells in the experiment group were smaller than those in the control group. In vitro wound healing kinetic showed that the hyperoxia/hypoxia stimulation compromised HUVEC wound closure; transwell migration assay confirmed that migrated cells were more in the control group, indicating truncated ability of migration in the experiment group.

\section{Conclusion}

The proliferation and migration abilities of endothelial cells are damaged in the experiment group, which are consistent with the inhibition of angiogenesis of ROP in early stage. Therefore, in light of the rat OIR model, the cycled hyperoxia/hypoxia cell model has potential in mimicking ROP vascular injury.

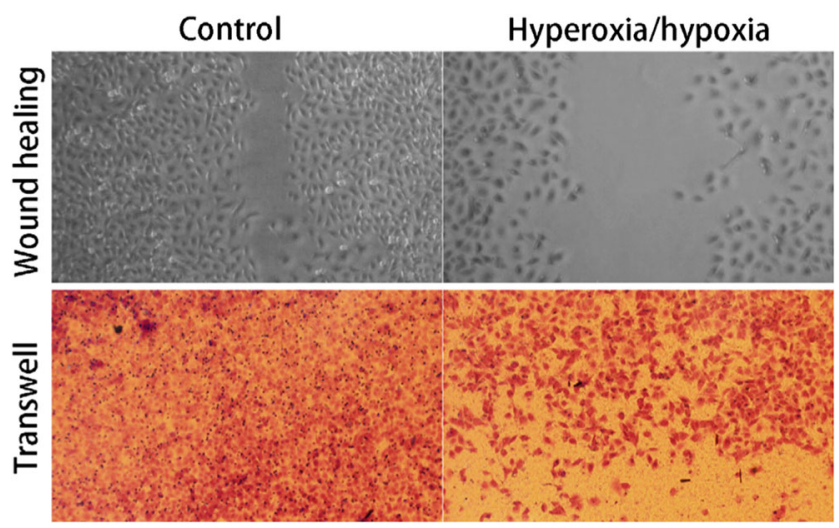

632

\section{Nephrology}

IL-6 Receptor Gene Polymorphism in Idiopathic Nephrotic Syndrome in Kuwaiti Children

Amal A. Al-Eisa ${ }^{1}$, Mohammad Z. Haider ${ }^{1}$

${ }^{T}$ Departement of Pediatrics, Kuwait university-Faculty of Medicine, Kuwait

\section{Background}

Idiopathic Nephrotic syndrome (INS) is an immune-mediated disease in which the many cytokines are involved. IL- 6 is a cytokine found to be associated with mesangial diffuse glomerulonephritis and chronic kidney disease. Gene polymorphism of IL-6 receptor (IL-6R) controls the expression and function of IL-6 and may affect the pattern of INS.

\section{Objective}

The aim of this study is to determine the frequency and the association of IL-6R gene polymorphisms with idiopathic nephrotic syndrome (INS) and its effect on the disease pattern in Kuwaiti children.

Methods

Genotypes of the IL-6R gene polymorphisms were analyzed using PCRRFLP in 156 INS patients and 58 age and sex- matched controls. Clinical data of all subjects were reviewed.

\section{Results}

A total of 156 INS (131 steroid -sensitive SS\&25 steroid resistance SR) patients with a mean age was $7.3 \pm 4.0$ years were studied. Male: Female ratio was $2: 1$. The CC genotype of IL-6R gene polymorphism was detected in $75 \%$ of the INS patients compared to $69 \%$ of the controls $(P=0.47)$. The heterozygous GC genotype was detected in $19 \%$ of INS patients compared $27.5 \%$ of the controls $(P$ $=0.25)$. The GG-genotype was detected in $5.7 \%$ of INS patients and $3.5 \%$ of the controls $(P=0.73)$. The $\mathrm{C}$-allele frequency in homozygous and heterozygous forms was found in $94 \%$ of INS patients compared to $96.5 \%$ of the controls $(P=0.73)$. The $\mathrm{G}-$ allele frequency in homozygous and heterozygous forms was found in $25 \%$ of INS patients compared to $31 \%$ of the controls $(P=0.47)$. No significant difference was found in any of the allele frequencies between SS and \& SR sub-groups when compared with each other or when compared to the controls.

\section{Conclusion}

Our data shows no role of IL-6 receptor gene polymorphisms on the clinical pattern or response to steroids in Kuwaiti Arab Children with INS.

\section{1}

\section{Nephrology}

A case of early recurrence of focal segmental glomerulosclerosis after renal transplant

Adriana Monica Bungardi ${ }^{1}$, Aurel Bizol, ${ }^{1,2}$, Cornel Aldea ${ }^{l}$, Bogdan Bulata' $^{\text {, Mihai Lucan }}{ }^{3,4}$, Dan Gheban ${ }^{5}$, Dan Delean ${ }^{l}$

${ }^{1}$ Nephrology and Dialysis Department, Emergency Clinic Hospital for Children, Romania

${ }^{2}$ Pediatric Nephrology Department, University of Medicine and Pharmacy "Iuliu Hatieganu”, Romania

${ }^{3}$ Renal Transplant Department, Urology and Renal Transplant Institute, Romania ${ }^{4}$ Urology Department, University of Medicine and Pharmacy "Iuliu Hatieganu", Romania

${ }^{5}$ Department of Anatomical Pathology, Emergency Clinic Hospital for Children, Romania

\section{Introduction}

Primary focal segmental glomerulosclerosis (FSGS) is one of the most severe glomerular diseases, with multifactorial and often undefined pathogenesis. It presents with proteinuria, usually of nephrotic range, and progression to end-stage renal disease (ESRD) is frequent.

FSGS is the initial renal disease in approximately one-tenth of pediatric patients receiving a renal allograft. In the first renal transplantation, the nephrotic syndrome and progressive glomerular sclerosis recur in 30 to $40 \%$ of patients, which negatively impact graft survival.

\section{Case-report}

We present the case of a 16-year-old Caucasian girl with primary FSGS, which debuted as a pure nephrotic syndrome, refractory to corticosteroids and other immunosuppressive agents. It slowly progressed to ESRD, for which she necessitated chronic hemodialysis until the possibility of transplantation. After the renal transplant from a deceased donor, which occurred 4 years after the onset of FSGS, there were immediate complications: a lymphocele and a subcapsular hematoma, leading to acute tubular necrosis. Due to the urological reintervention, plasmapheresis and immunosuppressive treatment, the renal function rapidly improved. Two months after the transplantation, the laboratory tests revealed impaired renal function with nephrotic range proteinuria. It was interpreted as an early recurrence of FSGS, uninfluenced by medical therapy or plasmapheresis, requiring allograft removal and resumption of chronic hemodialysis.

\section{Conclusion}

Compared to other renal diseases, the incidence of delayed graft function is higher and graft survival is lower in children with FSGS. At the present time, most researchers consider FSGS as a podocyte disease, but the pathogenesis of recurrent FSGS is far from being established. 
In spite of the risk of recurrence and the therapy for FSGS in renal graft being largely empirical, patients with FSGS should not be excluded from transplantation.

\section{3}

Nephrology

\section{HIV related kidney diseases}

Mihai Gafencu $^{1,2,3}$, Faris Liem Milhem ${ }^{2}$, Rodica Costa ${ }^{2}$, Gabriela Doros $^{1,2}$

${ }^{1}$ Pediatrics, UNIV. MED PHARM V. BABES Timisoara, Romania

${ }^{2}$ Pediatrics 4, UMF, Emergency Children Hospital, Romania

${ }^{3}$ Pediatrics, UMFT, Romania

\section{Introduction}

Since the earliest description of HIV infection, renal involvement were reported. Renal disorders are encountered at all stages of HIV infection, and they range from the imbalances commonly seen in hospitalized patients to end-stage renal disease (ESRD). We refer an estimated 10,000 children infected in Romania, from1987-1990. children Cohort.

\section{Objectives}

Study prevalence, gender, viral load, renal pathologies, mortality of HIV patients with renal manifestations in western Romania.

\section{Material}

The children admitted in Timisoara children hospital between 1994 2015, from a total patients with HIV: 640 and those with renal manifestations (albuminuria, malformations, ESDR). Methods for detection a renal disorder: Addis count, Albuminuria, Urine culture, 24 urine collection, Serum creatinine and Creatinine clearance.

\section{Results}

We analise age of the onset of HIV infection, type of kidney involvement, in conection with antiretroviral therapy and modality of transmission, stage at the monent of presentation in hospital, Viral load and CD4+ count, and renal parameters. Kidney involvement was noticed in $21 \%$ of the cases $(65 \%$ Females, $35 \%$ Males). Ninety percent acquired the disease from parenteral route of a blood product and syringe reuse. Twenty-six percent deaths from 135 with kidney involvement (73 \% died between the years 1994-1999). UTI among deaths was the most common pathology 24 out of the 35 mortal cases. Conclusions

Ninety percent acquired the virus via nosocomial way of transmission mainly by parenteral route of a blood product and syringe reuse. From eight different types of renal involvement the urinary tract infection was by far the most common involvement (E. coli $61 \%$ ). Sixty-three percent of the patients were stage $\mathrm{C}$ as per $\mathrm{CDC}$ staging, with $\mathrm{C} 1$ and $\mathrm{C} 3$ being the most prevalent. Mortality rate of $26 \%$, much higher than it is displayed in another studies. Albuminuria was present within deaths in $(53 \%)$ of the cases, in living patients (15\%) of the patients.

\section{8}

\section{Nephrology}

\section{Treatment of hypophosphatemic rickets in children}

Alexandra Kaplina ${ }^{1}$

${ }^{T}$ Department of Pediatrics, Saint-Petersburg State Pediatric Medical University, Russia

\section{Background}

Hypophosphatemic rickets (HR) is a hereditary disease, characterized by hypophosphatemia, phosphaturia, reduced tubular reabsorbtion of phosphate (TRP\%), low maximal tubular phosphate reabsorbtion per glomerular filtration (TmP/GFR). HR includes autosomal dominant (ADHR), recessive (ARHR), X-linked HR, hypophosphatemic bone disease (HBD), hereditary HR with hypercalciuria (HHRH). Patients with HR need a lifelong phosphate replacement therapy.

\section{Objective}

To analyze the effect of phosphate replacement and active vitamin D therapy in children with HR.

\section{Methods}

Twenty eight children (for aged 2 to 16 years; 18 girls, 10 boys) with HR were included in the study. They have been receiving phosphate, active vitamin $\mathrm{D}$ therapy for 7 years (median duration). Children were followed 6 monthly. The outcome measures: height changes; TRP\%, TmP/GFR (Schwartz), fractional excretion of phosphate (FEP\%), urinary $\mathrm{P} / \mathrm{Cr}, \mathrm{Ca} /$ $\mathrm{Cr}$; serum phosphate and alkaline phosphatase (ALP) levels.

\section{Results}

The patients were matched by HR type (genealogical method): ADHR, Xlinked HR - 13 children, HBD - 5, ARHR - 6, HHRH - 4. HR children had impaired height at the baseline, which increased to age norm in 9 (32.1\%) patients (mostly ADHR). ADHR, X-linked HR children had an increase of TRP to $75.1 \pm 10.3 \%$ (baseline $70.3 \pm 15.8 \% ; \mathrm{M} \pm \mathrm{SD}$ ). ALP decreased to $835.4 \pm 442.5$ (from $1055.9 \pm 347.4$ ) at the outcome. Patients with HBD had slightly changed serum phosphate levels, $\mathrm{P} / \mathrm{Cr}$ at baseline; TRP normalized. ARHR children had the highest ALP $(1173.5 \pm 243.4)$, low TRP $(70.3 \pm 12.5 \%)$ in outcome. HHRH patients weren't administered active vitamin $\mathrm{D}$. They had TmP/GFR increased $(0.47 \pm 0.2)$, FEP significantly decreased $(19.5 \pm 8.5 ; p=0.044)$ from baseline. HR patients had increased FEP in outcome. Seven children had metaphyseal-deaphyseal osteotomy to correct leg deformities: ADHR, X-linked HR - 3, ARHR - 3, HHRH - 1.

\section{Conclusion}

Phosphate replacement therapy had a positive effect on height, ALP, TRP in children with HR. The biggest effect had been in patients with ADHR, X-linked HR. HBD had a favorable course, patients didn't need surgical treatment.

\section{1}

Nephrology

Nutritional Assessment for Children With end-stage Renal Disease on Regular Dialysis (Khartoum- Sudan)

Amin Alagib Mohamed Musa ${ }^{l}$

${ }^{T}$ Pediatrics, Ass.Professor.Pediatrics, Sudan

- Talal A. AbdelRahman Ibrahim (1)

- Amin Alagib M.Musa,. (1)

- Dr. Rashid A. Ellidir.(2)

- Nassan M. Ahmed(1)

- R. Brai

\section{Background}

Chronic kidney disease (CKD) describes the gradual loss of kidney function. It has five stages; the last stage is referred to as end-stage renal disease that requires renal replacement therapy (i.e. renal dialysis or transplantation). Malnutrition is a common complication of chronic kidney disease and is associated with increased morbidity and mortality in children and adults.

\section{Objective}

The aim of this study is to assess the nutritional status of the children with ESRD on regular dialysis attending Noura center for kidney disease and renal transplantation in Soba University Hospital Khartoum.

\section{Methods}

A cross-sectional descriptive Analytical study was done at Noura center for kidney disease and renal transplantation in Soba University Hospital. All the patients attending the dialysis sessions there were included in this study $(n=40)$ aged from 3 to 18 years. Weight, height, total plasma protein levels and serum albumin levels were measured.

\section{Results}

Height was the most affected anthropometric parameter with $69.8 \%$ were stunted, older children were affected more than younger ones and no significant association between the serum albumin level and the presence of wasting or stunting.

\section{Conclusion}

The prevalence of malnutrition in our patients with end stage renal disease (ESRD).was high (30\% among the 40 children included in this study) There was no specific age group affected more than the other; and no 
significant association was found between serum albumin levels and the presence of malnutrition or stunting. There were only three patient peritoneal dialysis and were trained to perform it at home.

They just come for follow up every mouth.

\section{4}

\section{Nephrology}

The relationship between urinary tract infection and vesicourethral reflux in children from eastern galilee region

Wael Nasser ${ }^{l}$, Shabtai Brill ${ }^{l}$, Ehsan Nasser ${ }^{l}$, Sami Shehadi ${ }^{l}$, Suzan Nasser ${ }^{l}$ ${ }^{T}$ Pediatric Nephrology, Baruch Padeh Medical Center, Faculty of Medicine in the Galilee - Bar Ilan University., Israel

\section{Introduction}

Urinary tract infection(UTI) is the most common bacterial infection among children. Upper UTI involves renal parenchyma and can cause scar tissue formation in the kidney that may lead to hypertension and renal function insufficiency.

Vesicourtereral reflux waspreviosly considered as a contributer to UTI incidence and thus to renal scaring. Therefore, Cystography, which is considered as the "gold standard" for reflux detection, was performed often in children despite exposure to radiation and inconvenient inherent in it.

\section{Methods}

This retrospective study was designed to determine whether cystography is a necessary part of upper UTI in children, by exploring the relationship between the presence of vesicourtereral reflux (reflux) and UTI.

The study enrolled 88 children, after verified UTI, who underwent cystography procedure. Sixty-nine of them also underwent DMSA mapping.

\section{Results}

About $40 \%$ of children after UTI were diagnosed with reflux by cystography.

Nearly $70 \%$ of children with pathologic DMSA mapping were diagnosed with reflux.

Moreover, children with reflux were also found to have elevated inflammation factors, comparing to those without reflux.

\section{Conclusion}

Based on our results, DMSA can become a Suitable replacement procedure to Cystography since it can detect renal scarring with good outcomes relative to cystography, and is much easier to perform on a child.

We can also conclude that reflux is associated with infection due to higher levels of inflammation factors (ESR, CRP) in correlation with reflux presence. This study has a vast importance in the consolidation of protocols and guidelines regarding the procedures suitable for children after UTI.

433

\section{Nephrology}

Renal findings in Tuberous Sclerosis Complex: A Three Case Report Alexandra Virginia Sobek ${ }^{l}$, Andreia Nita ${ }^{l}$, Irina Dijmarescu ${ }^{l}$, Mihai Romascanu $^{I}$, Laura Balanescu ${ }^{1,2}$, Oraseanu Dumitru ${ }^{1,2}$, Daniela Pacurar $^{1,2}$

${ }^{1}$ Pediatrics, "Grigore Alexandrescu" Emergency Hospital for Children from, Romania

${ }^{2}$ Pediatrics, Carol Davila University of Medicine and Pharmacy, Romania

\section{Background}

Renal involvement is a major cause of morbidity and mortality in tuberous sclerosis complex (TSC). The most common renal lesions associated with TSC are angiomyolipomas (AMLs), cysts and renal cell carcinoma. Renal cysts and AMLs usually do not grow significantly until after puberty, and often not until the third or fourth decade of life.

Objective

The aim of our paper is to highlight the diversity of renal manifestations of TSC in children and the importance of periodic imaging surveillance.

\section{Methods}

We present three cases of TSC admitted to our hospital with different kidney findings.

Results

Case 1, a 12-year-old boy was admitted for abdominal pain. He was diagnosed with TSC at 18 months of age: lumbar Shagreen patch, hypomelanotic macules, facial angiofibromas, subcortical and subependymal hamartomas, subependymal giant cell astrocytoma. The abdominal computerized tomography (CT) scan showed multiple cysts and hypodense nodules, homogenous, some with fat content, $8 / 6 \mathrm{~mm}$, well-defined, in both kidneys. Case 2, a 16-years-old boy, diagnosed with TSC at the age of 13 (hypomelanotic macules, facial angiofibromas, periungual and gingival fibromas, subependymal hamartomas), was admitted for reevaluation: abdominal CT scan describes multiple hepatic cysts and multiple hypodense images, $16 / 14 \mathrm{~mm}$, well-defined, that disorganize normal structure in both kidneys (AMLs). Case 3, a 9-year-old boy, diagnosed with TSC at 1 year of age (cutaneous findings), was admitted for an abdominal mass $(15 / 10 \mathrm{~cm})$ in the left hypochondrium. The abdominal CT scan revealed a non-homogeneous mass in the left kidney, with an irregular outline, $12 / 7 \mathrm{~cm}$, compressing the pyelocaliceal system (AML). All patients have renal function intact. The renal lesions were discovered by routine ultrasound.

\section{Conclusion}

Renal manifestations vary a lot in TSC: from small to gigantic AMLs, without correlation with age or other TSC findings. These 3 different cases outline the importance of routine ultrasound in children with TSC.

477

\section{Nephrology}

Nephrogenic diabetes insipidus - case report of a family from Romania

Ramona Stroescu $^{1,2}$, Mihai Gafencu $^{1,2}$, Adela Chirita ${ }^{1,2}$, Raluca Isac ${ }^{1,2}$, Gabriela Doros ${ }^{1,2}$

${ }^{1}$ Pediatrics, "Louis Țurcanu” Emergency Hospital for Children, Romania

${ }^{2}$ Pediatrics, University of Medicine and Pharmacy "Victor Babeș", Romania

\section{Introduction}

Nephrogenic diabetes insipidus is a rare $\mathrm{X}$ linked syndrome, secondary to mutations in the gene encoding the vasopressin V2 receptor, located at position Xq28. Aim: To present a family with nephrogenic diabetes affecting three males in two generations.

\section{Material and method}

We report the cases of a 4 month old patient, male, admitted in our hospital for growth impairment, vomiting and fever, and his 10 year old uncle, admitted at the same time for excessive fluid intake (11 liter/day). Results

Family history (two generations affected; uncle's brother died at the age of two with severe hypernatremic dehydration), polyuria $7.8 \mathrm{ml} / \mathrm{kg} / \mathrm{h}$, lab work-up (plasma hyperosmolarity, hypernatremic hyperchloremic alkalosis, extremely low urine density (1002), elevated ADH levels) were suggestive for nephrogenic diabetes insipidus. Treatment with hydrochlorothiazide was initiated $(2 \mathrm{mg} / \mathrm{kg} /$ body $)$, with reduction in urine output $(2.85 \mathrm{ml} / \mathrm{kg} / \mathrm{h})$, but severe consecutive alkalosis and hypokalemia in the infant. An aldosterone antagonist (spironolactone) was added, with good response. Uncle's fluid intake was reduced to 3-4 1/day, while urine output decreased to $31 /$ day.

\section{Discussion}

Diabetes Insipidus includes a large series of disorders. Many of the known disorders are now susceptible to symptomatic treatments or specific interventions such as dietary modification, thiazides and inhibitors of prostaglandin synthesis. However, these approaches can only ameliorate the clinical phenotype. In infants, treatment is particularly difficult. It aims to avoid episodes of dehydration and obtain a normal growth curve. 


\section{Conclusions}

This highlights the importance of early diagnosis of diabetes insipidus in infants with persistent hypernatremia

554

Nephrology

Macedonian Enuretic Study Group (MESD) - Mission and Challenges

Velibor Tasic ${ }^{1}$, Nadica Ristoska-Bojkovska ${ }^{2}$

${ }^{T}$ Pediatric Nephrology, University Children's Hospital Skopje, Macedonia

${ }^{2}$ Pediatric Dept., Clinical Hospital Acibaden Sistina Skopje, Macedonia

The establishment of the Macedonian Enuresis Study Group (MESD) resulted from the need to increase the knowledge and awareness among the health professionals about this topic since epidemiologic data on the prevalence are scarce; there is lack of knowledge about the attitude of the physicians and families about the enuresis/incontinence, treatment modalities and outcomes. This is a non-formal internet based group with voluntary participation mainly of pediatricians, general practitioners urologists and other health professionals who manage children with enuresis/incontinence. The basic mission of the group is to connect the pediatricians and other health professionals who work on the problem of the enuresis/incontinence in children and adolescents; to disseminate/spread the knowledge on this topic in order to improve management of these patients and to support research and scientific activities on the local and international level. Spread of the knowledge should be done trough (i) Papers, articles, book chapters (ii) Regular Pubmed abstracts (iii) Guidelines (iv) Other materials (v) Discussion of difficult to solve cases. The group will initiate collaborative studies and for each study/project core group of 4-5 members will be elected. They will design the study protocol and create appropriate questionnaire. Ethical approval is not mandatory for observational/noninterventional study. We have such approval at the University Children's Hospital. We expect realization of the first study until the end of 2017 year.

\section{6}

\section{Nephrology}

Voiding Cystourethrography - Do We Over-Do IT

Dragana Zivkovic ${ }^{l}$, Dragan Sarac ${ }^{1}$, Ivana Fratric ${ }^{1}$

${ }^{T}$ Pediatric Urology, Institute For Child and Youth Health Care of Vojvodina, Serbia

\section{Background}

Voiding cistourethrography (VCUG) is the commonly used modality in the setting of (febrile, upper, or neonatal) urinary tract unfection - not in the primary assessment but after the infection has been treated, to assess for vesicoureteric reflux, but also in diagnostics of other urogenital anomalies. Objective

To determine if there is a way to lower the number of VCUG-s performed. Methods

Retrospective study was conducted with approval of the Ethics Review Board using clinical data of the patients who underwent VCUG over the period of 12 months. Indicators (age of the patient, gender, referring specialist - Urologist or Nephrologists and indications for VUCG) were analyzed using Chi-square (Table 1), Fisher's exact and Mann-Whitney test. Possible impact of the above mentioned indicators on the result of the VCUG were analyzed using logistic regressive analysis.

\section{Results}

Total of 197 VCUGs were analyzed. Mann-Whitney U test did not show statistically significant age difference between the patients with normal and pathologic findings on VCUG $(Z=-0.415, p=0.678$, median age in patients with normal finding was 3 years and in patients with pathologic findings on VCUG was 2.5 years). Chi-square test showed that patients with single urinary infection and other indications have a higher chance of normal result on VCUG ( 0.041 and 0.011 respectively) unlike patients with repeated VCUG who have a higher chance of pathologic findings (0.000). Logistic regressive analysis showed that patients whose referring specialist was urologist had 2.06 times higher chance for having pathologic finding on VCUG $(b=-0.810, p=0.007$, odds ratio $=0.445)$ compared to those referred by Nephrologist.

Conclusion

At our Institute Pediatric Urologists indicate VCUG more cautiously compared to the Pediatric Nephrologists. By avoiding VCUG after single urinary tract infection and for other indications we could reduce the number of VCUGs performed.

\section{1}

Neurology

Association Between Iron Deficiency Anaemia and Febrile Seizures in Children Attending a Tertiary Care Centre- a Case Control Study Mohammad Shah Alam ${ }^{1}$, Mohammed Rezaul Karim ${ }^{2}$, Kamrun Nahar ${ }^{3}$ ${ }^{T}$ Department of Paediatrics, Upazila Health Complex, Bangladesh ${ }^{2}$ Department of Paediatrics, Coxsbazar Medical College, Bangladesh ${ }^{3}$ Department of Pharmacology, Chittagong Medical College, Bangladesh

\section{Background}

Febrile seizures are the most common seizures in children. It is prevalent in children aged between 6 months and 60 months, with an incidence of $2-5 \%$. On the other hand iron deficiency anaemia is the most common hematologic disease of children with a period of incidence that coincides with the time of developing febrile seizures. Therefore, it is hypothesized that there is a possible association between this two conditions.

\section{Objective}

The purpose of this study was to determine the association between Iron deficiency anaemia and febrile seizures in children.

\section{Methods}

In this case-control study 80 children with a diagnosis of febrile seizures, aged between 6 months and 60 months who were visited Pediatric ward of Chittagong Medical College Hospital, Chittagong, Bangladesh during June 2012 to may 2013 were enrolled as cases. The control group consisted of 80 febrile children without seizure and were matched to the cases by gender and age. Iron deficiency anaemia was diagnosed by hemoglobin value $11 \mathrm{gm} \%$, serum ferritin value $30 \mathrm{ng} / \mathrm{mL}$ and RDW $15 \%$.

\section{Results}

A total 36 out of $80(45 \%)$ cases had iron deficiency anaemia whereas, 08 out of $80(10 \%)$ controls were found to have iron deficiency anaemia. There was statistical significance of difference observed in two groups $(\mathrm{P}$ 0.001). Univariate risk factor analysis for control versus case showed odds ratio $7.364(3.138$ - 17.279) $(95 \%$ CI). The mean serum ferritin level of cases and controls were $43.01 \pm 29.77$ and $72.36 \pm 38.66(\mathrm{ng} / \mathrm{mL})$ respectively ( $\mathrm{P}$ 0.001). The mean value of $\mathrm{HB} \%, \mathrm{MCV}, \mathrm{MCH}$, and RDW\% were also found significantly lower in the cases than controls $(\mathrm{P}$ 0.001).

\section{Conclusion}

Iron deficiency anaemia was more frequent among children with febrile seizures than those with febrile illness alone.The findings of this study suggest a positive association between iron deficiency and febrile seizures in children and screening for Iron deficiency anaemia should be considered in children presenting with febrile seizures. Early detection and timely correction of iron deficiency anaemia may prevent febrile seizures in children.

\section{7}

\section{Neurology}

Mind over Matter?... Referral of Patients with Symptoms of NonOrganic Origin to a Tertiary Inpatient Paediatric Neurology Service Deirdre Foley ${ }^{1}$, Rosemarie Manning ${ }^{I}$

${ }^{T}$ Paediatric Neurology Department, OLCHC, Ireland 


\section{Background}

The paediatric neurology department in OLCHC receives an average of twelve new referrals for inpatient neurology consultation per week. We found that many of these patients were subsequently diagnosed with a non-organic illness or a psychosomatic cause for their symptoms.

\section{Objective}

To assess the proportion of neurology referrals which had an underlying non-organic cause. To carefully qualify and quantify the type and number of investigations performed prior to diagnosis of these patients, and services involved in subsequent management.

\section{Methods}

Retrospective audit of inpatient neurology referrals over a six-week period was performed. Ethical approval was obtained. Patients with presentation of non-organic origin were identified and the course of their hospital stay assessed. Outcomes were measured in terms of investigations performed, services involved, and time to diagnosis.

\section{Results}

Of $n=73$ new patients reviewed over a six week period, we identified $n=15$ patients for inclusion in our final cohort. Eighty-seven percent of patients had a co-morbid organic illness. A trigger for symptom onset was identified in $60 \%$ of patients. Reasons for neurology referral included pseudoseizure, collapse, abnormal eye movements and gait abnormalities. MRI brain was performed in $40 \%$ of patients and EEG in $60 \%$. The clinical psychology service was involved in $53 \%$ of our cohort, and medical social work services in $13 \%$. Parents were in support of the diagnosis made in $73 \%$ of cases, by time of discharge.

\section{Conclusion}

Though non-organic illness may present in a wide variety of ways, we found that it represented a significant proportion of our team workload. We recommend 1. Education sessions for healthcare professionals to encourage early identification of non-organic illness 2 . A hospital wide protocol for management of non-organic illness once diagnosed, including early cessation of unnecessary investigation 3 . Early input from psychology services and allied health professionals.

\section{8}

Neurology

Incidence and prevalence rate of Segawa disease in Japan Hiroki Fujioka $^{1,2}$, Takashi Hamazaki ${ }^{2}$, Tomato Sakaguchi ${ }^{2}$, Haruo Shintaku

${ }^{I}$ Department of Health Science, Kansai University of Welfare Sciences, Japan ${ }^{2}$ Department of Pediatrics, Osaka City University Graduate School of Medicine, Japan

\section{Background}

Segawa disease is a very rare inherited disease, which is characterized by dopa-responsive dystonia and diurnal fluctuation. Dysfunction of GTP cyclohydrolase I (GTPCHI) due to mutations of the enzyme-coding gene, $\mathrm{GCH}$, reduced the production of neopterin and biopterin, and this induced a shortage of dopamine in the central nervous system.

\section{Method}

We examined 137 patients who suffered dystonia and/or other involuntary movements, from January 2012 to December 2016. We measured the neopterin and biopterin content in these patients. Genetic analysis of GCH1, which encoded GTPCHI, was also performed in 50 patients.

\section{Results}

Twenty-two patients had mutations in $\mathrm{GCH} 1$ and were diagnosed with Segawa disease. These patients comprised 18 females and 4 males. The average age of patients who underwent genetic analysis was 19.35 years (Range: 7 to 62). Most of the patients who were diagnosed as adults showed dystonic symptoms from a very young age. The mutations detected in the $\mathrm{GCHI}$ of patients in this study included six missense mutations, three frame-shift mutations, one nonsense mutation, four mutations in introns around exons, and four large exon deletions. Three families had their own unique mutation; however, no common mutation was observed in other patients. In this study, we examined patients from 32 of the 47 prefectures in Japan (total population of these areas was 106,058,400 in 2016). The calculated incidence rate of Segawa disease was 4.1/ $100,000,000$ per year in this study. The prevalence rate was considered $1.6 / 1,000,000$ when the average of duration of this disease was 40 years. Conclusion

Over the course of five years, we genetically diagnosed patients with Segawa disease. No common mutation was observed. The incidence rate was 4.1 per hundred million people per year and the prevalence rate was 1.6 per million people in Japan.

734

\section{Neurology}

Audit of compliance with Tuberous Sclerosis guidelines in a paediatric cohort attending Cork University Hospital

Susan Harvey ${ }^{I}$, Olivia O'Mahony ${ }^{1}$, Niamh McSweeney ${ }^{I}$

${ }^{7}$ Department of Paediatric Neurology, Cork University Hospital, Ireland

\section{Background}

Tuberous sclerosis, an autosomal dominant condition occurring in 1 in 6000 live births, is caused by a mutation in either the TSC1 or TSC2 gene. It is characterized by the presence of hamartomas in the brain, heart, skin, kidneys or lungs. Disease related complications include developmental delay, epilepsy and behavioural difficulties. Management Guidelines were produced in 2012 by an International Consortium[i].

\section{Methods}

We undertook a retrospective chart review auditing compliance with international tuberous sclerosis recommendations.

\section{Results}

There were 11 patients attending the paediatric neurology service at time of this review, Four of eight patients tested had the TSC2 mutation, 1 had the TSC1 mutation. Nine patients had an EEG with seven patients requiring ongoing anti-epileptic treatment. Nine patients had an echocardiogram, three had cardiac rhabdomyomas. Two patients had a diagnosis of an Autism spectrum disorder. Eighty one per cent of patients had a MRI Brain within the last three years as per recommendations. Seven MRI brains showed features consistent with tuberous sclerosis such as subependymal nodules and white matter lesions. Two cases had SEGAs, one had been resected. Ten patients had renal ultrasounds within the last three years, two patients had bilateral angiomyolipomas and one patient had polycystic kidney disease. No patient was documented as having attended a dentist within the previous year. One patient had attended an ophthalmologist.

\section{Discussion}

Our centre showed good complicance with imaging and genetic recommendations however poor compliance with cardiac, ophthalmological and dental follow up recommendations. This may be related to availability of resources within our hospital. Formal structured follow up is required to ensure optimal care of these patients.

\section{5}

\section{Neurology}

\section{Review of paediatric headache referrals to a regional centre Susan Harvey ${ }^{1}$, Niamh McSweeney ${ }^{I}$ ${ }^{T}$ Department of Paediatric Neurology, Cork University Hospital, Ireland}

\section{Background}

Headaches are a common reason for referral to paediatric clinics. We reviewed the type of headache referrals received with the aim of using this information to triage more effectively and better use available resources to lead to better patient outcomes.

Methods

We undertook a retrospective chart review of all patients referred with a primary headache over a six-month period from January to June 2016. 


\section{Results}

Thirty-five patients with a mean age of 10.1 years (3-15.7 yrs) were referred over the six-month period, $18 / 35$ to the neurology service. Nine patients were referred with red flag symptoms including nocturnal or early morning headaches, vomiting or progressive worsening in headaches. The presence or absence of red flag features was omitted in 12 referrals. Relevant family history was included in $17 \%$ of referrals. Clinical exam details were absent in $26 \%$ of referrals. On average patients waited 124.2 days for their appointment. Those with red flag symptoms waited on average 108.6 days. Twelve patients (34\%) were referred for imaging and 9/10 MRIs performed were normal (34\%). One patient was found to have two incidental ependymal cysts in the right lateral ventricle. Of the patients with a headache variant diagnosis, classical migraine was the most common at $45 \%$, followed by tension headache at $28 \%$. Lifestyle modification was the most common advice, prophylaxis included Riboflavin and Pizotifen and acute treatment with triptans. Conclusion

Low patient referral numbers may be accounted for by patients accessing services through the emergency department or the Paediatric Assessment Unit. As referral information was often suboptimal, we aim to introduce a template for referral for primary care colleagues in order to correctly identify patients in most need of rapid access to paediatric services.

\section{1}

\section{Neurology}

A young boy with recurrent episodes of vomiting: the importance of early diagnosis

Maria Loureiro ${ }^{I}$, Mariana Pinto $^{1}$, Magalys Sotto ${ }^{1}$

${ }^{T}$ Pediatrics, Centro Hospitalar Entre Douro e Vouga, Portugal

\section{Introduction}

Papillary tumor of the pineal region (PTPR) is a newly described entity, which has been recently included in the World Health Organization classification of central nervous system tumors.

Case-report

A 5-year-old boy presented twice, in 72 hours, to the emergency department with vomiting with no other symptoms. Was discharged with antiemetic treatment. He returns on the 4th day of illness with headache, vomiting and diplopia. Radiologic studies revealed triventricular hydrocephalus secondary to a space-occupying lesion in the pineal region. Endoscopic third ventriculostomy was performed to treat hydrocephalus and the lesion was biopsied. Complete resection of the tumor was subsequently performed. The diagnosis of PTPR was established on immunohistopathological examination. Postoperative radiotherapy resulted in complete regression of the tumor with no evidence of tumor recurrence at 12 months.

\section{Conclusion}

Papillary tumors of the pineal region constitute a rare entity, with a difficult diagnosis, an uncertain prognosis, and a high risk of local recurrence. They must be treated by a combination of surgery and radiotherapy. This case shows the importance of a detailed clinical history and physical examination to a child presenting with symptoms of intracranial hypertension.

177

Neurology

Basic principles rightfully governing the design of tools in autism: a physicians's perspective

Amalia Megremi ${ }^{l}$, Ioannis Darzentas ${ }^{l}$

${ }^{T}$ Product and System Design Engineering, University of Aegean, Greece

\section{Background}

Effective therapy of autism does not exist. So, it is urgency for tools that could contribute to functional autonomy of autistics.
Objective

Formulation of principles that govern the design of tools in autism.

Methods

Literature review

Results

Individualization: There are autistics who get easily bored, so the stimulation must be high in order to maintain their attention, and others who are disturbed by the simultaneous presence of different stimuli. Therefore, tools must be dynamic in order to compensate for changing patterns of autistics. Complexity: Autistics have lost their complexity and behave in a uniform, predictable, stereotypic manner. The aim is to learn the concept of accepting the unpredictable governing the real world. Gradation: The complexity that will be provided for learning should be gradually enriched, so that it can be accepted by the autistics. Controlled Flexibility: The tool should give the complexity of the situation to be learned the possibility either to increase (if the procedure learning progresses) or decrease (if it does not progress). Familiarity: Autistics have excellent resistance to unfamiliar experiences. So, tools must include activities familiar to them. Repeatability: The tool must provide the possibility of repetition (autistics learn better in a ritually stereotyped manner). Generalization: Generalization (in autism lacks-deficit in theory of mind) is the capacity to enable the newly-acquired abilities in the real world. Self-control: The tool must enable individuals to watch themselves performing tasks in which they have been trained (activation of mirror neurons, whose defectiveness in autism is significant). Human Factor: The human factor when using tools to acquire skills (trainer's voice, gestures, presence of peers) is necessary so that the "autistic individual does not become more autistic".

\section{Conclusions}

Maintenance of these principles could contribute to the effectiveness of tools for autistics and to the achievement of functional autonomy of the latter.

696

\section{Neurology}

\section{Case Report}

Headaches and Finding of Lipoma Intraduralis Intramedularis Regio C1/C4

Dubravka Mitrović ${ }^{1}$, Tijana Lalićl, Vera Vujovic $^{l}$

'Primary health center "Dr Simo Milosevic" Cukarica, Belgrade, Serbia

\section{Introduction}

Headaches in children are very common symptom. About $75 \%$ of children had a headache at least once a year, while $30 \%$ have recurrent headaches. They can be primary, inorganic and secondary, organic. The secondary may be caused by brain trauma, vascular disease and brain tumors. Brain tumors are the second most frequent in children after leukemia. The incidence is 4 per 100000 children. Good history and early diagnosis are the most important for the outcome of the disease.

Case

A girl aged 9 coming to the examination because of a knock to the head. There were no signs of brain contusion. Even before the knock, the girl was complaining of periodical headaches treated by ibuprofen. She was born with mild hypotonia (CNS Ultra Saund and EEG examinations were normal), due to which the physical treatment was performed during the first 5 years of life. Myopia was diagnosed at age of seven. Neurological examination didn't reveal pathological deficiencies. MRI shows expansive intradural lesion $(52 \times 27 \times 19 \mathrm{~mm})$ of craniocaudal extension from the Framen Ovale Magnum up to the level $\mathrm{C} 4$, in the dorsal part of the spinal canal. The mutation compresses the medulla but without the signs of myelomalacia and cerebrospinal fluid flow obstruction. The girl has been operated and the $\mathrm{PH}$ findings showed that it was an intramedullary lipoma,a very rare primary benign tumor. Follow-up MRI of endocranium and cervical spine showed present remains of lipoma $(45 \times 21 \times 15 \mathrm{~mm})$ with milder degree of medulla compression. 


\section{Conclusion}

Intradural intramedullary tumors represent $35 \%$ of all spinal tumors in childhood period. Intradural lipomas are even rarer and constitute only $1 \%$ of spinal cord tumours. Monitoring of headaches in the childhood period is necessary.MRI diagnostics is reasonable even when there are no neurological disorders and symptomatic seizures. Proper anamnesis is a base for further diagnostics.

452

Neurology

An Audit of Biotinidase Deficiency among Children and Young People In a Scottish NHS Region between 2014 And 2016

Michael Ogundele ${ }^{I}$, Abdulbaset Noaman ${ }^{1}$, Heather Holmes ${ }^{2}$, Mary Jarvie ${ }^{2}$

${ }^{T}$ Community Paediatric Unit, NHS Fife, UK

${ }^{2}$ Clinical Biochemistry Department, NHS Fife, UK

\section{Background}

Some evidence-based guidelines have recommended biotinidase deficiency (BD) screening.for investigating children with moderate or severe global developmental delay. patients with residual BT activities $1 \%$ (partial $\mathrm{BD}$ ) may remain asymptomatic even without treatment.

\section{Objectives}

We aimed to analyze the pattern and outcome of investigations for BTD among Community Pediatricians in a Mid-Eastern Scottish NHS Area.

Method

We retrospectively reviewed the clinical and laboratory data of all children throughout NHS Fife area screened for Biotinidase activity (BTA) over a two- year period between July 2014 and July 2016.

Biotinidase activity was measured in plasma using a colourimetric assay, by Spectrophotometric methods. Profound biotinidase deficiency is defined as levels $0.7 \mathrm{nmol} / \mathrm{min} / \mathrm{mL}$ and partial deficiency between 0.7 and 2.1. Newborn infants in the partial deficient range should have repeat samples taken when they are $3-6$ months of age to determine their status. Results

A total of 262 tests were requested for 243 children aged between 1 and 153 months (Mean 70 months, SD 42 months, Median 60 months). Fifteen patients had their samples repeated once and two patients had their samples tested twice. Five patients had BTA values below the reference value of 4 . One of them on repeat increased from 2.7 to 3 , while the other increased from 2.7 to 8.7 within two months. The remaining 3 patients had BTA values between 3 and 4 which were not repeated. Results of 191 patients were analysed with BTA ranging between 3 and 14.1. None of the patients had BT deficiency.

\section{Conclusion}

None of the patients studied had abnormal BTA results. However a substantial proportion of the patients' samples 53/244 (22\%) could not be analysed in the laboratory due to various problems. A larger prospective study is required to verify the true prevalence of BTD in the population.

458

Neurology

Analysis of Laboratory Samples Rejection For Biotinidase Deficiency (BTD) Screening among Children And Young People in a Mid-Eastern Scottish LA

Michael Ogundele ${ }^{1}$, Abdulbaset Noaman ${ }^{1}$, Heather Holmes ${ }^{2}$, Mary

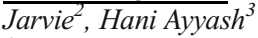

${ }^{1}$ Community Paediatric Unit, NHS Fife, UK

${ }^{2}$ Clinical Biochemistry Department, NHS Fife, UK

${ }^{3}$ Paediatric Neurodevelopmental Service, 1Cambridgeshire and Peterborough NHS Foundation Trust, University of Cambridge Health Teaching NHS Trust, UK

\section{Background}

Specimens submitted to the Pathology laboratories are usually rejected as unsuitable for analysis for various reasons at an approximate mean rate of $0.3 \%$ and up to $0.75 \%$, but higher values of up to $13 \%$ have also been reported. Pre-analytical errors largely related with procedures outside the laboratory are usually the commonest causes of rejection but the majority of them are preventable.

\section{Method}

Laboratory and clinical data of all patients screened for BTD over a twoyear period across NHS Fife were retrospectively analyzed. All the blood specimens for Biotinidase tests were sent to a central laboratory at Edinburgh for analysis. Research Ethics Committee approval was not required, as this was a retrospective audit using routinely collected anonymised data from the laboratory computer system.

Results

NHS Fife is one of the fourteen regions of NHS Scotland. Fife is a large rural area with a total estimated population of 368,280 (Mid 2015), of which $17.4 \%$ are children aged 0 to 15 years old 64081 .

A total of 262 tests (equivalent to 2 per 1000 per year) were requested for 243 children aged between 1 and 153 months (Mean 70 months). Seventy-five samples were ordered to be repeated. Fifteen patients had their samples repeated once and two patients had their samples tested twice, giving a repeat sampling rate of 19/75 (25\%). Various reasons for repeat testing, the commonest being "Insufficient sample" (Table 1). Fifty-six samples from 53 (22\%) patients were unsuitable for analysis. None of the 191 patients' samples analysed had abnormal BTA results.

\section{Conclusion}

There is an exceptional high rate of sample rejection and low rates of resampling for Biotinidase screening tests, well above the universally reported levels. Further studies are required to better understand the reasons for this observation and to devise strategies to reduce these incidents in the future.

Table 1 showing various reason for rejection of the blood samples

\begin{tabular}{|r|r|l|}
\hline Percent & $\begin{array}{r}\text { No of } \\
\text { samples }\end{array}$ & Reasons for rejection \\
\hline 54.7 & 41 & Insuificient \\
\hline 17.3 & 13 & $\begin{array}{l}\text { Unsuitable, Heparin specimen } \\
\text { required. }\end{array}$ \\
\hline 8 & 6 & Test missed in error \\
\hline 4 & 3 & Leaked in transit \\
\hline 2.7 & 2 & Low Values \\
\hline 1.3 & 1 & No specimen received \\
\hline 2.7 & 2 & Mislabeled \\
\hline 2.7 & 2 & Test not indicated \\
\hline 1.3 & 1 & Unsuitable \\
\hline 1.3 & 1 & Regret request missed \\
\hline 4 & 3 & No Reasons \\
\hline Total & 75 & \\
\hline
\end{tabular}

832

Neurology

A National Survey of Community Paediatric Services for Management of Neurodevelopmental, Neurobehavioural, Psychological and Psychiatric Problems

Michael Ogundele ${ }^{2}$, Hani Ayyash ${ }^{1}$, Tanja-Sabine Schumm ${ }^{3}$, Richard Lynn ${ }^{4}$, Michael Morton ${ }^{3}$

${ }^{1}$ Integrated Child Health Department, Cambridgeshire and Peterborough NHS Foundation Trust, UK

${ }^{2}$ Community Paediatrics Unit, Glenwood Health Centre,, NHS Fife, UK

${ }^{3}$ Institute of Health and Wellbeing, University of Glasgow, UK

${ }^{4}$ British Paediatric Surveillance Unit, Royal College of Paediatrics and Child Health, UK 


\section{Background}

There is a wide variation about services configuration and ${ }^{1}$ management provided for children and young people (CYP) with common Neurodevelopmental, Psychological and Psychiatric problems across the UK. This survey was conducted as part of a validation survey of the Child and Adolescent Psychiatrists Surveillance System (CAPSS), carried out to ascertain its feasibility and acceptability for active monitoring of rare psychiatric childhood conditions.

Aims

We aimed to determine the extent of involvement of Community Paediatricians in management of Childhood Mental Disorders.

Methods

A questionnaire was designed for Community Paediatricians by modification of a similar CAPSS survey questionnaire. Community Paediatric staff were invited to participate in the survey via a link to the web-based tool Survey Monkey. Responses were obtained between Dec 2015 and August 2016. All survey responses were transferred to Microsoft Excel for analysis.

\section{Results}

A total of 245 respondents working within 180 Community Paediatric units across the UK completed the online questionnaires. This included Consultants (75\%), Specialist Associates/Staff Grades (19.5\%) and others including Trainees (5\%).

The majority of the respondents worked with children and young people from $0-18$ years-(97\%). $11.6 \%$ of them worked with older people aged 19-25 years, especially those with complex disabilities who attended special schools, where no specific adult services existed or where there were problems or delays with transition to adult services. Table 1 shows the different areas of Community Child Health involvement of the respondents. The commonest areas of Community Child Health involvement include Neurodevelopmental (70 \%), Neurodisability $(50 \%)$ and Safeguarding (43\%).

\section{Conclusion}

Responses highlight the wide range of work Community Paediatricians are expected to take on in the field of CYP mental health. Most of the Community Paediatricians highlighted the challenges of service provision for young people above 16 years and the desirability of jointworking with CAMHS.

Table 1 showing different areas of Community Child Health involvement of the respondents

\begin{tabular}{|l|c|c|}
\hline Areas of involvement & Percent (\%) & $\begin{array}{c}\text { No. } \\
\text { Respondents }\end{array}$ \\
\hline Neurodevelopmental (including ADHD and ASD) & $70.48 \%$ & 160 \\
\hline Neurodisability - Epilepsy, sleep, sensory & $50.22 \%$ & 114 \\
\hline Safeguarding/Child Protection & $42.73 \%$ & 97 \\
\hline Behavioural Paediatrics & $32.60 \%$ & 74 \\
\hline Looked After Children & $29.96 \%$ & 68 \\
\hline Foetal Alcohol Syndrome & $17.62 \%$ & 40 \\
\hline $\begin{array}{l}\text { Other (e.g Genetics, Research, Continence, SEND, } \\
\text { Palliative) }\end{array}$ & $22.91 \%$ & 52 \\
\hline
\end{tabular}

684

Neurology

Severe Vitamin B12 Deficiency in an Exclusively Breastfed 9-monthold Infant

M. Pesmatzoglou ${ }^{l}$, E. Botsa $^{I}$, H. Angelopoulou $^{l}$, E. Dimitrakopoulou ${ }^{l}$, A. Korbaki ${ }^{1}$, I. Orfanou

${ }^{1}$ Children's Hospital Agia Sofia, 1st Pediatric Clinic University of Athens, Greece

\section{Background}

Vitamin B12 deficiency is an important and in lots of cases an under recognized cause of neurological morbidity in infants. The main cause of vitamin B12 deficiency in exclusively breastfed infants is maternal deficiency.

\section{Objective}

We present an infant with hypotonia, developmental retardation and cerebral atrophy due to vitamin B12 deficiency.

\section{Case presentation}

An exclusively breastfed 9-month-old female infant, who was born after a normal full-term pregnancy, was hospitalized due to failure to thrive, apathy, hypotonia, developmental retardation and cerebral atrophy on MRI scan. The child showed normal developmental features up to 6 months, but after then the parents recognized that their baby became less active, stopped communicating and had refusal of solid food. Upon admission, her weight, height and head circumference were dropped down more than 2 major percentile lines, she was hypotonic, apathetic, lacking smiling and presenting involuntary movements. She had a hemoglobin level of 9,1g/dl with an MCV of 99,5fl. The results of metabolic examinations revealed elevations in Methylmalonic Acid $(183.4 \mathrm{mmol} / \mathrm{mol}$ creatinine, normal value 2.5$)$ and Homocysteine $(157.90 \mu \mathrm{mol} / \mathrm{L}$, normal value 6.10-9.22). Furthermore, her serum vitamin B12 level was $50 \mathrm{pg} / \mathrm{ml}$ (normal value 211-911) and serum folate level $20 \mathrm{ng} / \mathrm{ml}$ (normal value 4.6-18.7). Her mother's vitamin B12 level was $163 \mathrm{pg} / \mathrm{ml}$. The child was diagnosed as having vitamin B12 deficiency due to maternal deficiency and was treated with intramuscular vitamin B12 injections. After the initiation of vitamin B12, the infant developed severe involuntary movements and seizures. Antiepileptics were introduced in addition to Vitamin B12 and seizures disappeared. After a few days clinical improvement was observed with an achievement of developmental milestones, but she still showed significant delayed neuropsychiatric development.

\section{Conclusion}

Vitamin B12 deficiency should be considered in all infants with developmental retardation and hypotonia because early identification and treatment can prevent irreversible brain injury.

\section{0}

\section{Neurology}

Infantile Tremor secondary to Vitamin B12 deficiency: Case report and Literature review

Bronwyn Power ${ }^{1}$, Suzanne Slattery ${ }^{1}$, Bryan Lynch ${ }^{1}$

${ }^{T}$ Dept. of Neurology, Children's University Hospital, Temple Street, Ireland

\section{Background}

Adverse neurological outcomes have been associated with Vitamin B12 (Cobalamin) deficiency in infancy. The exact aetiology is unclear, but likely multifactorial. Interference with normal myelination, deranged sadenosylmethionine production and aberrant cytokine regulation are postulated mechanisms in the literature. ${ }^{1}$ Neurological manifestations of infantile B12 deficiency include poor feeding, hypotonia, developmental delay, tremor and seizures. Nutritional vitamin B12 deficiency is a treatable cause of neurologic dysfunction in infants.

\section{Aim}

We report an interesting case of tremor in an exclusively breastfed infant secondary to maternal Vitamin B12 deficiency.

\section{Methods}

The clinical findings, radiological imaging and blood profile of the index case were reviewed. The current available literature on vitamin B12 deficiency in the infantile period was evaluated.

Results

A 7 month term baby boy presented with a 2 month history of intermittent, symmetrical tremulous movements of his head, trunk and upper limbs. Neurological examination was unremarkable. Initial investigations including FBC, MCV, renal, liver, bone profiles and EEG were within normal limits. Urine organic acids revealed an elevated urinary methylmalonic acid (MMA) (266 umol/mmol (0-8umol/mmol)). Serum homocysteine was also raised $(26 \mathrm{mmol} / \mathrm{l}(0-11))$. Serum Vitamin B12 was low (127 ng/L (210-760)), with a serum folate (24 ug/l) within the normal range. His mother had a non-vegan diet, but was deficient in Vitamin B12 (163 ng/L). 


\section{Conclusion}

This case outlines a rare presentation of infantile tremor associated with functional Vitamin B12 deficiency. Maternal dietary deficiency is the most common cause of Vitamin B12 deficiency in infancy. ${ }^{1}$ Methylmalonic acid and Homocysteine are more sensitive and specific indicators of vitamin B12 status than Mean Corpuscular Volume (MCV). ${ }^{2}$ Prompt diagnosis and management of Vitamin B12 deficiency is essential to prevent significant irreversible neurological sequelae.

1. Smith J, Coman D (2014) Vitamin B12 Deficiency: an Update for the General Paediatrician. Pediat Therapeut 4: 188.

2. Rasmussen SA, Fernhoff PM, Scanlon KS. Vitamin B12 deficiency in children and adolescents. J Pediatr 2001;138:10-7

\section{9}

\section{Neurology}

TSC2/PKD1 contiguous gene deletion syndrome

Niamh Ryan ${ }^{1}$, Donncha Hanrahan ${ }^{2}$, Conor Bogue ${ }^{3}$, Maria Stack $^{4}$, Niamh McSweeney ${ }^{I}$

${ }^{1}$ Department of Paediatric Neurology, Cork University Hospital, Ireland ${ }^{2}$ Department of Paediatric Neurology, The Royal Belfast Hospital for Sick Children, Ireland

${ }^{3}$ Department of Paediatric Radiology, Cork University Hospital, Ireland ${ }^{4}$ Department of Paediatric Nephrology, Temple Street Children's University Hospital, Ireland

\section{Introduction}

Tuberous Sclerosis(TS) is an autosomal dominant disorder due to mutations in either TSC1 encoding hamartin or TSC2 encoding tuberin. Both genes play important roles in normal functioning of the mammalian target of rapamycin (mTor pathway) and mutations in this pathway causes abnormal cellular proliferation and differentiation producing hamartomatous lesions. PKD 1 and TSC2 genes lie tail to tail on Chromosome 16p13.3. Deletions in this region lead to TSC2/ADPKD1 contiguous gene syndrome. We report two cases of de novo TSC2/PKD1 contiguous gene deletion syndrome

Case1

A male infant presented at 13 weeks with focal seizures, global developmental delay, macrocephaly and bilateral ballotable kidneys. Abdominal ultrasound confirmed extensive cystic replacement of renal parenchyma. MRI Brain showed multiple subependymal nodules and tubers.

He had a de novo large deletion of the TSC2 gene from exon 1-41 to at least exon 46 of the PKD 1 gene. He has refractory focal epilepsy and chronic Hypertension with normal renal function. At nine months he has an evolving right hemiplegia and significant Developmental Delay.

Case 2

Case 2 presented at 3 months with supraventricular tachycardia and has upper lobe congenital emphysema. He had a large deletion of TSC2/ADPKD gene. CT brain was suggestive of TS. At 5 months he had focal seizures with EEG showing temperoparietal discharges. At 8 months he developed clinical and electrographic spasms and responded to Vigabatrin.He is on Everolimus for multicystic kidney disease.

\section{Conclusion}

Only 33 cases of TS and ADPKD have been described in the literature. Renal complications are common in TS, however the coexistence of these two genes leads to increased rate of malignancy and development of ESRF at an earlier age. Our cases highlight the treatment challenges that these patients pose including seizure control and antihypertensive management.

687

\section{Neurology}

To follow head or heart, an interesting case presentation Samantha Slee $^{I}$, Jasavanth Basavaraju ${ }^{I}$, Hemalata Bentur ${ }^{I}$, Maysara Abdelaziz ${ }^{I}$

${ }^{1}$ Paediatric Department, Whiston Hospital, St Helens and Knowsley Teaching Hospitals NHS Trust, UK

\section{Background}

The most important diagnostic problem in epileptology is to distinguish epileptic seizures from syncope. The diagnosis of episodic altered consciousness rests largely with the clinical history and neurophysiology. We present an interesting case of a 12-year-old boy who was initially treated for epilepsy.

\section{Objective}

To review the timeline of events and how the diagnostic conclusion was made in association with the history of seizures.

\section{Methods}

Retrospective review of the patients' medical records.

Results

A 12-year-old boy was assessed in epilepsy clinic following a generalised tonic clonic seizure. He had possible recurrent seizures as a toddler and had been seizure free for 8 years. A clinical diagnosis of epilepsy was considered. ECG was normal; MRI showed an incidental sub-cortical right inferior frontal lobe cyst. During standard EEG, on photic stimulation, he had a generalised tonic clonic seizure; the ECG recording on the EEG was noted to have a progressive bradycardia leading to a 6 seconds pause preceding the seizure, with no epileptiform activity. The diagnosis of epilepsy was revised to a diagnosis of a possible dysrhythmia. A 72hour ECG recording was performed; during an incidental venepuncture procedure he had an 8 second pause on his ECG, culminating with a tonic clonic seizure. A diagnosis of severe neurocardiogenic syncope was made and he was commenced on Atropine 500micrograms TDS. Due to the infrequency of seizures the patient will be followed up as an outpatient to make a decision on whether he will require pacemaker insertion.

\section{Conclusion}

Neurocardiogenic syncope can present as a seizure disorder and history alone may not be sufficient to make a confident diagnosis, which can be difficult. Careful interpretation of relevant investigations may aid in making the right diagnosis; our case demonstrates the importance of interpreting the simultaneous ECG on an EEG recording.

\section{2}

\section{Neurology}

N-methyl-D-aspartate-receptor Encephalitis: Case Series Ruth Anne Tugawin ${ }^{1}$, Ruth Anne Tugawin ${ }^{1}$, Maria Franchesca Bueno ${ }^{1}$ ${ }^{T}$ Pediatrics, Baguio General Hospital and Medical Center, Philippines

\section{Introduction}

Anti-N-methyl-D-aspartate receptor encephalitis is a rare cause of encephalitis which was previously thought to be viral encephalitis. This rare illness may initially present as seizure episodes, psychiatric manifestations or an involuntary movement disorders which were observed in 8 different pediatric patients presented in this case series report.

\section{Objectives}

To present a case series of autoimmune encephalitis with different manifestations. To discuss the case management, prognosis and updates of this type of encephalitis.

\section{Case Reports}

We describe 8 cases of encephalitis presenting with different initial symptoms, 4 cases presented initially with psychiatric manifestations, 2 with movement disorder and 2 presented with seizure. Seven of the cases later develop seizure episodes at home. During hospital stay all of the patients manifested orofacial dyskinesias and choreoathetoid movements.

\section{Discussions}

Autoimmune conditions are not a common cause of encephalitis. However, it is imperative that a diagnosis of Autoimmune encephalitis be considered when other more common causes have been excluded or when there are certain diagnostic indicators such as presence of prodromal syndrome, orofacial dyskinesias, faciobranchial dystonic seizures, catatonia or extrapyramidal signs which were all seen in the 8 cases. Because of a strong clinical suspicion and following the course of the disease, the diagnosis of autoimmune encephalitis was highly considered. Treatment with 
Methylprednisolone is imperative once autoimmune encephalitis is entertained even in the absence of antibody titers or while awaiting for the result of autoimmune antibody screening. Six patients were started on Methylprednisolone with noted improvement 7 days after treatment, however two patient died due to hospital acquired infection, one patient went home against medical advice. Treatment with Steroids plus IVIG has been shown to improve outcome, however only three patient had IVIG treatment. Conclusions

The diagnosis of anti-NMDAR encephalitis is challenging especially if the initial presentation can also present in other more common condition like CNS infection. A prompt diagnosis and early treatment with immunotherapy decreases mortality.

Keywords

autoimmune, encephalitis, N-methyl-D-aspartate, orofacial dyskinesia

\section{5}

\section{Obesity}

Epidemiologic and Nutritional profile of children in public schools Jane Cardoso ${ }^{I}$, Mayara Rozante ${ }^{I}$

${ }^{T}$ Medicine, University Center Maringa, Brazil

\section{Introduction}

The increase in obesity among children is a concern of great importance in public health once studies have shown it reflects in a higher risk of obesity in adulthood and comorbidities.

\section{Objective}

Describe the nutritional and epidemiologic profile of children in public schools in an urban area in Southern Brazil.

\section{Method}

Cross-section study using convenience sample of 568 children ages 5 to 10, from day care facilities and public schools in Southern Brazil. Data regarding weight, height and Body Mass Index (BMI) was collected, according to Health Ministry Technical Normative. The nutritional status was defined and weight, height and BMI's Z score averages were obtained, by age and sex. Height by age (H/A) and BMI/A of pre-schoolers and schoolchildren were compared.

Results

Fifty-one percent are females and $49 \%$ are males. The age average was 6.17. Males and females had a weight average of $23 \mathrm{~kg}$ and height average of $117 \mathrm{~cm}$. In regard to nutritional status, $70 \%$ of children were eutrophic; $27 \%$ of females and $23.5 \%$ of males were overweight, obese or severely obese, considering that males $(4.9 \%)$ presented almost twice the rate of severe obesity compared to females (2.9\%). Comparing preschoolers (ages 5 to 6 ) to schoolchildren (ages 7 to 10 ), $23 \%$ and $56 \%$, respectively, were overweight, obese and severely obese.

\section{Conclusion}

Most children had adequate growth rates, however there is high prevalence of overweight, obesity and severe obesity, twice as much among schoolchildren, which draws attention to the necessity of intervention in form of nutritional control in schoolchildren.

142

\section{Obesity}

Association of BMI with motor performance in undernourished Santal children of Purulia district, West Bengal, India

Sutanu Dutta Chowdhury ${ }^{1}$, Tusharkanti Ghosh ${ }^{2}$

${ }^{T}$ Department of Physiology, Basirhat College, India

${ }^{2}$ Department of Physiology, University of Calcutta, India

Children's motor development is closely associated with their adiposity status. Association between adiposity in terms of BMI and motor performance has been established in children and adults by few researchers. These results were observed in children from nontribal population, BMI related differences in motor skills have not been studied in tribal children and specifically in Santal children. The present study was undertaken to assess the adiposity status by measuring body mass index (BMI) and to investigate the influence of BMI on motor performance in 5-12 years aged Santal children. This study was conducted on 816 Santal children. The adiposity status of each child was assessed by BMI-for-age z-score based on WHO reference data. Motor development was measured using the Bruininks-Oseretsky Test of Motor Proficiency (BOT-2). Mean BMI values of Santal children remained around the 50th percentile values of WHO reference data. The normal weight (NW) children scored higher in some individual motor subtests (bilateral coordination, balance, running speed and agility, upper limb coordination, and strength) and in total BOT-2 score (p0.05) compared to that of underweight (UW) and overweight (OW) children. A significant association was observed between distribution of children in BOT-2 z-scores categories and BMI-for-age z-score categories $\left(\mathrm{X}^{2}=71.36\right.$, df $\left.=3, \mathrm{p} 0.01\right)$. Stepwise regression analyses showed that BMI have significant impact on motor performance of Santal children. Motor performance showed a curvilinear relationship with BMI as the motor scores found lower in both UW and OW children compared to NW children. The results also indicated that gross motor skills are more affected with the change of BMI in comparison to fine motor skills.

\section{5}

Obesity

\section{Stress Induced Obesidome}

Styliani Geronikolou $^{1,2}$, Athanassia Pavlopoulou ${ }^{4}$, Konstantinos Albanopoulos $^{3}$, Dennis Cokkinos ${ }^{l}$, George Chrousos ${ }^{1,2}$

${ }^{1}$ Clinical, Translational, Experimental Surgery Research Center, Biomedical Research Foundation of the Academy of Athens, Greece

${ }^{2}$ First Dpt of Pediatrics Aghia Sophia Children Hospital, Athens University Medical School, Greece

${ }^{3}$ First Dpt of Propedeutic Surgery, Hippokrateion Hospital, Athens University Medical School, Greece

${ }^{4}$ Computer Science and Biomedical Informatics, University of Thessaly, Greece

\section{Background}

The pathogenesis of chronic-stress related obesity epidemic includes effects on Hypothalamus-Pituitary-Adrenal axis released hormones, visceral fat accumulation as a result of chronic hypercortisolism, reactive insulin hyper secretion, low growth hormone secretion and hypogonadism. The dominant pathogenic mechanism is that of oxidative stress and research interest on antioxidant genes dominates the scientific community. Aim

To construct an interactome of stress induced obesity, in the view of advancing the insight of the pathogenesis of the disease.

\section{Methods}

Obesity-related gene or gene products were extracted from the biomedical literature (Patterson and Abizaid, 2013, Qi and Cho, 2008). The interactions among them were investigated through STRING v10 (Szklarczyk et al., 2015), a database of known and predicted, physical and indirect associations among genes/proteins. In this study, a high confidence interaction score of 0.7-0.97 was selected.

\section{Results}

The intermediate nodes were also predicted, showing no more than 30 nodes of gene- gene products, illustrating known as well as predicted interactions (i.e. GHRL decreases $U C P 1$ and $U C P 2$ expression.

\section{Conclusions}

Interactions networks may help our better understanding of the pathogenesis of the disease. Moreover, CRH-AVP and INS (in the liver) are identified as major "hubs" for the stress induced (epigenetic) obesity. 
479

Obesity

Cardio-vascular health in normoweight and overweight adolescent girls in Greece

Styliani Geronikolou $^{1,2}$, Stavros Chrysanthopoulos ${ }^{1}$, Flora Bacopoulou $^{1,2}$, Dennis Cokkinos ${ }^{1}$

${ }^{1}$ Clinical, Translational, Experimental Surgery Research Center, Biomedical Research Foundation of the Academy of Athens, Greece

${ }^{2} 2$ Center for Adolescent Medicine and UNESCO Chair on Adolescent Health Care, First Department of Pediatrics, Athens University Medical School, Greece

\section{Aim}

To investigate potential cardiac and vascular differences between normoweight and overweight female adolescents.

\section{Methods}

Normoweight $(\mathrm{BMI}=23,1 \pm 2,3)$ and overweight $(\mathrm{BMI}=28,4 \pm 4,1)$ adolescent girls up to the age of 23 years, who attended the Center for Adolescent Medicine and UNESCO Chair on Adolescent Health Care, over a period of one year, were eligible to participate in the study. Cardiovascular assessment of each study participant was performed at the Clinical Research Centre of the Biomedical Research Foundation of the Academy of Athens, with the use of: a. Task Force Monitor for the baroreflex sensitivity (BRS) and time domain parameters of heart rate variability as sensitive markers of cardiac function, b. Vivid 7 for intima media thickness of carotid arteries.

\section{Results}

Forty one adolescent girls aged 13-23 years were enrolled in the study. No differences in cardiac or vascular marker was detected between normoweight and overweight participants ( $\mathrm{p} 0.05)$, but in baroreflex sensitivity $(p=0.01)$.

\section{Conclusions}

Changes in vascular pathophysiology associated to fat excess do not seem to manifest early in adolescence. On the contrary, BRS in this female adolescents specific population study, shows significant difference between the two weight groups. As this marker depends on age, BMI, arterial pressure, waist circumference, abdominal fat extensive body composition and vascular study is suggested.

\section{8}

Obesity

Associations between infant growth and later outcomes: does the choice of growth reference matter?

Taran Nandra ${ }^{1,2}$, Jane Williams ${ }^{2}$, Mary Fewtrell ${ }^{2}$, Katherine Kennedy ${ }^{2}$

${ }^{T}$ Faculty of Life Sciences and Medicine, King's College London, UK

${ }^{2}$ Nutrition Unit, Great Ormond St Hospital Institute of Child Health,

University College London, UK

\section{Background}

Use of different infant growth references (GR) can alter apparent infant growth pattern, but the impact on associations between early growth and later outcomes is unknown.

\section{Objectives}

To examine whether the choice of GR influences (1) apparent infant growth patterns; and (2) associations with later anthropometry and body composition (BC).

Methods

Weight, height, BMI, waist circumference (WC)) and BC (four component model: fat mass (FM), fat free mass (FFM)) were measured in 209 healthy British children (mean (SD) 11.1 (3.5) yrs, 104 boys). Infant weight data were extracted from parent-held child records for birth, 3, 6 and $12 \mathrm{wk}, 6$ and $12 \mathrm{mo}$ and converted to SDS using UK90 reference and WHO growth standards. $\Delta$ wtSDS using the two GRs were compared and associations between $\Delta$ weight $3-6 \mathrm{wk}$ and $0-12 \mathrm{mo}$ and later measurements were assessed, adjusting for confounders.

\section{Results}

$\Delta w t S D S$ calculated using UK90 and WHO were significantly different at all ages. UK90 $\Delta$ wtSDS $0-3 w k$ was significantly 0 and $\Delta$ wtSDS 3-6wk significantly 0 . WHO SDS were significantly 0 for $\Delta$ wtSDS 3-6wk and significantly 0 for $12 \mathrm{wk}-6 \mathrm{mo}, 6-12 \mathrm{mo}$ and $-12 \mathrm{mo}$. In boys, $\Delta \mathrm{wtSDS} 3-$ 6 wk was positively associated with later height, weight and FFM regardless of GR although associations were stronger for WHO than UK90 (eg. $0.65 \mathrm{WHO}(p=0.02), 0.57 \mathrm{UK} 90(p=0.04))$. In girls, $\Delta \mathrm{wtSDS} 3-6 \mathrm{wk}$ was positively associated with later FM and fat-free mass index (FMI) using both UK90 and WHO, and with weight, BMI, WC only using UK90; all associations were stronger using UK90 than WHO. $\Delta$ wtSDS birth-12mo in boys and girls was significantly associated with all later outcomes except FMI, with similar effect sizes using UK90 and WHO.

\section{Conclusion}

Differences in infant growth patterns as a result of using different GRs may influence apparent associations with later outcomes: effects may differ by gender and growth period. This should be considered by researchers.

651

Obesity

The waist-to-height ratio of eleven aged school children in Serbiapilot study

Tijana Lalic $^{1}$, Dubravka Mitrovic ${ }^{l}$, Milica Tasic ${ }^{l}$, Marija Aleksijevic ${ }^{1}$, ${ }_{\text {Vera Vujovic }}{ }^{\text {, Jelena Milin }}{ }^{2}$

${ }^{1}$ Primary health center "Dr Simo Milosevic", MD, Serbia

${ }^{2}$ School of medicine, Belgrade University, Institute for statistics, $M D$, Serbia

\section{Introduction}

The waist-to-height ratio (WHtR), calculated by dividing the waist circumference (WC) by height is newer anthropometric index. A WHtR cutoff 0.5 is generally accepted as a universal cut-off value for central obesity in children (aged $\geq 6$ years) and adults. It represents a screening tool in detecting children with higher cardiometabolic risk. More and more school children have abdominal obesity, and children are less physically active.

\section{Objective}

Application of WHtR for the first time and valuation regardless to nutritional status and physical activity of school children

Method

At an primary health office during physical examination of 183 children (94 boys and 87 girls) aged $10.9 \pm 0.4$ weight, height, WC were measured, and BMI and WHtR were calculated. The data about the recreational and active coaching physical activity of children was recorded. Statistical analysis were performed.

\section{Results}

The mean value WHtR was $0.48 \pm 0.17$, for boys $0.51 \pm 0.23$ and girls $0.46 \pm 0.07$. There is no statistically significant difference among them. Average values of WHtR are very similar $(p=0.159)$ between the children who exercise WHtR $0.50 \pm 0.25$, and which are not physically active WHtR $0.46 \pm 0.07$. WHtR values were similar ( $p=0.464$ ) between 38 normal weight WHtR $0.49 \pm 0.32$, and 31 overweight-obese boys WHtR $0.53 \pm 0.06$. While, in girls there is a statistically significant difference (p 0.001 ) between 54 normal weight WHtR $0.44 \pm 0.05$ and 23 overweight-obese girls WHtR $0.52 \pm 0.07$.

\section{Conclusion}

In this pilot study, the WHtR average value for school children of this age corresponds to WHtR cut-off value, regardless of gender, which is still good but represents a warning in the same time. Usually, obese and overweight children are encouraged to exercise more, especially boys. This could be the explanation for the obtained similarity. Additional studies are required but there is the unquestionable benefit of WHtR as a screening tool. 
275

\section{Obesity}

Prevalence of obesity in children with autism- A West Wales Study Hesham Nasef ${ }^{1,2,3}$, Qumrun Nahar ${ }^{1,2,3}$, Alice Setti ${ }^{3}$

${ }^{T}$ Paediatrics, West Wales General Hospital, UK

${ }^{2}$ Paediatrics, Hywel Dda university health board, UK

${ }^{3}$ Paediatrics, Hywel Dda university health board, UK

\section{Introduction}

- Feeding habits, behaviour and associated medical conditions are different in children with autism.

- Anecdotally based on our local assessment of children with autism, we felt there is an increase in prevalence of obesity.

- The study aim is to estimate the current prevalence of obesity in children with autism.

- The audit results can be helpful in constructing designated guidelines for the management of obese children with autism

Methods

- Retrospective data collection.

- A snapshot of children with autism presented to our outpatient department during the period $01 / 01 / 2014$ to $31 / 12 / 2014$.

- 39 notes analysed.

\section{Results}

Twenty percent of the study sample are overweight or obese.

Fifty percent of the overweight or obese were girls while $14 \%$ were boys Nearly $80 \%$ has comorbidities.

Physical activity was not explored during history taking in any overweight or obese child.

None were given advice regarding weight loss programs.

Twenty-five percent were referred to a dietician or a specialist

\section{Conclusion}

- Various studies report the prevalence of obesity in autism to be between

23 to $30 \%$. Our study shows similar prevalence $(20 \%)$

- Interestingly, we found a comparatively higher prevalence of obesity in girls $(50 \%)$ as compared to boys (14\%).

- The above findings would imply that obesity is prevalent in children with autism and would require suitable management.

- It should be specially recognized that Autistic girls would, in particular, need careful attention to their weight gain issues.

\section{Recommendation}

- Prompt recognition of obesity in children with autism with early intervention

- There is a need for construction of local guidelines for management of autistic children who are overweight or obese with multidisciplinary team involvement

\section{0}

\section{Obesity}

Secular Changes in Waist Circumference, Waist-to-Height Ratio and Body Mass Index in Thai Children

Lakkana Rerksuppaphol ${ }^{1}$

${ }^{T}$ Department of Preventive Medicine, Faculty of Medicine, Srinakharinwirot University, Thailand

\section{Background}

Prevalence of childhood obesity has increased worldwide. Central obesity is an important predictor of the metabolic disorders and cardiovascular risk factors. Waist circumference (WC), waist-to-height ratio (WHtR) and body mass index (BMI) are the commonly used to define central fatness and obesity.

\section{Objective}

The study aimed to measure the secular changes and update the reference standards of these parameters for Thai children.

\section{Methods}

A cross-sectional survey of 3,885 school children 6.0-12.99 years of age in Ongkharak district of central Thailand was conducted in 2013. Weight, height and WC were recorded by trained staff using sensitive and calibrated instruments. BMI and WHtR were calculated by standard formulae. Age and gender specific smoothened percentile curves were created by using least mean squares method. The data was compared with that from a 2008 cohort from the same area.

\section{Results}

BMI, WC and WHtR of boys and girls were comparable at $18.0 \pm 4.4 \mathrm{vs}$ $17.8 \pm 4.4 \mathrm{~kg} / \mathrm{m}^{2}, 61.7 \pm 11.8$ vs $61.9 \pm 11.0 \mathrm{~cm}$ and $0.46 \pm 0.07 \mathrm{vs}$ $0.46 \pm 0.06$, respectively. BMI and WC increased but WHtR changed relatively little with age in both genders. In comparison to 2008, BMI were increased by $0.9-1.1 \mathrm{~kg} / \mathrm{m}^{2}$ in boys and by $0.8-1.1 \mathrm{~kg} / \mathrm{m}^{2}$ in girls. WC and WHtR have increased consistently across all age groups and both genders. The 75th percentile for WHtR corresponded closely to 0.50 in both genders which has been the suggested cut-off.

\section{Conclusion}

There have been significant increases in WC, WHtR and BMI across all age groups and in both genders in Thai children. These findings reflect increasing prevalence of central obesity and are indicators of potential increase in cardio-metabolic health problems in coming years. WC and WHtR should be routinely measured in pediatric examination for early diagnosis of central obesity.

\section{8}

\section{Obesity}

Clinical Aspects after Bariatric Procedures in Adolescent: Long Time Follow-Up

Nicola Zampieri ${ }^{1}$, Roberto Castellani ${ }^{2}$, Marta Peretti ${ }^{1}$, Federica Bianchi $^{I}$, Simone Patanè ${ }^{l}$, Francesco Camoglio ${ }^{I}$

${ }^{1}$ Department of Surgery, Azienda Ospedaliera Universitaria Intergata, University of Verona, Italy

${ }^{2}$ Department of Emergency and Surgery, Casa di Cura Dott.Pederzoli Hospital, Italy

\section{Introduction}

The role of bariatric surgery and its role in adolescent is still under discussion worldwide. The aim of this study is to report clinical outcomes of bariatric procedures in adolescents with a medium and long time followup.

\section{Materials and Methods}

We retrospectively analyzed consecutive data added into the Italian register of the society for bariatric surgery(period 2000-2012). We evaluated all patients treated with a mean follow-up of 3 years. Inclusion and exclusion criteria were created. All patients were aged between 13 and 18 yrs. We evaluated and compared clinical outcomes between procedures.

\section{Results}

After reviewing medical charts, 173 patients were considered for the study; 85 patients were treated with AGB, 47 with intragastric balloon, 26 with SG and other 15 patients with malassorbptive techniques. Among clinical data, there was a statistical difference in term of \%EWL between techniques only after 1 year post-op (p0.05); at 5 years, considering the $\%$ of patients studied, sleeve gastrectomy had the best \%EWL respect to other techniques ( $\mathrm{p} 0.05)$ and the best \%of improvement about comorbidities; at 5 year more than $90 \%$ resolved their comorbidities especially hypertension, dyspnea, orthopedic problems and dyspnea.

\section{Conclusions}

This study is the first reporting a National survey in adolescent; more than $80 \%$ of patients are followed till 4 years post-op but only few patients (less than $5 \%$ ) till 10 years. Our results demonstrated that sleeve 
gastrectomy in adolescent is safe and had a better \%EWL respect to other techniques with a good clinical outcomes.

\section{7}

\section{Paediatric Tropical Medicine}

Typhoid Fever In Young Children Of The North Indian Province Of Punjab

deepak bhat $^{1,2}$, Gurdeepsingh Dhooria ${ }^{1,2}$, Shruti Kakkar ${ }^{2}$

${ }^{T}$ pediatrics, dayanand medical college, India

${ }_{2}^{2}$ pediatrics, dayanand medical college, India

\section{Background}

In highly endemic countries like India, typhoid fever is the most common bacteremic illness. The clinical presentation of typhoid fever in infants and young children can be varied and non specific.Typhoidal illness can present as a mild,non specific febrile illness to severe illness with features of the classic typhoidal syndrome of high fever,toxicity,hepatomegaly and splenomegaly.

Objectives

To study the clinical profile of blood culture positive typhoid fever children living in on of the provinces of northern India.

Results

During the study period there were211 blood culture positive cases.Out of these 211 cases, $35(16.6 \%$ ) were in children less than 2 years.Male to female ratio was 1.7:1.Maximum number of cases $(43 \%)$ were seen during the months of July,August and September.All the patients (100\%) had fever at presentation,followed by diarrhea (48.5\%),vomiting (31.4\%).On examination anemia was present in $31(88.5 \%)$, hepatomegaly $(48.5 \%)$, splenomegaly $(25.7 \%)$.Maximum patients $(80 \%)$ had defervescence within first 7 days of starting antibiotics.On laboratory investigations ALT was raised in $30(85.7 \%)$ cases, LDH (31.4\%),Serology i.e. widal test(TO titres more than 160) was reactive in $6(17.1 \%)$ cases.Out of total 35 cases salmonella typhi was seen in $31(88.5 \%)$ and paratyphi in $4(11.5 \%)$ cases.Out of total 35 salmonella isolates all $(100 \%)$ were resistant to naldixic acid, $10(28.5 \%)$ were resistant to aminoglycosides, $2(5.7 \%)$ to fluroquinolones and $9(25.7 \%)$ to both.All patients were unimmunized against typhoid vaccine. 29 out of 35 patients were being bottle fed

\section{Conclusions}

In highly endemic countries like India,typhoid fever is one of the important reasons for admission to hospital in children less than 2 years age. One of the significant risk factors seen was compromised hygiene and bottle feeding was a big contributing factor.Now that conjugate typhoid vaccine is available,the inclusion of this vaccine in the national immunization schedule will go a long way in decreasing the disease burden in this age group.

\section{6}

\section{Paediatric Tropical Medicine}

Psychological Factors May Play an Important Role in Pediatric Functional Dyspepsia

Viacheslav Berezhnyi ${ }^{1}$, Maryna Mamenko ${ }^{1}$, Hanna Drokh ${ }^{l}$, Ivanna Romankevych ${ }^{1}$

${ }^{1}$ Pediatrics Dept.\# 2, Shupyk National Medical Academy of Postgraduate Education, Ukraine

\section{Background}

Dyspepsia is a common clinical condition associated with a complex of upper abdominal symptoms including: upper centered discomfort or pain, feeling of abdominal fullness, early satiety, abdominal distention and bloating, belching, 5.2 and nausea. Stress plays an important role in emergence of FD symptoms and sympathoadrenal imbalance. The most common comorbid psychological disorders seen in FGIDs include: anxiety disorders, mood disorders and somatoform disorders.

\section{The aim}

To investigate psychological disorders and sympathoadrenal imbalance (SB) condition in children with FD.

\section{Methods}

Target group: 80 schoolchildren aged 6-12 with FD. The study involved psychological testing and of SB assays (ELISA: cortisol, norepinephrine, insulin, adrenocorticotropic hormone (ACTH), glucose levels).

\section{Results}

$38.8 \pm 1.2 \%$ patients $(31 / 80)$ demonstrated clinical performance of isolated abdominal pain syndrome and $61.1 \pm 2.3 \%$ children $(49 / 80)$ had symptoms of post-prandial distress syndrome. Psychological testing revealed high levels of anxiety $-38.8 \pm 1.5 \%$ (31/80), depression $41.3 \pm 2.3 \%$ (33/80), sleep disorders $-85.0 \pm 1.1 \%$ (68/80) and cognitive dysfunction $-46.3 \pm 1.4 \%(37 / 80)$. The functional status of the sympathoadrenal system was as follows: 24-hour urine norepinephrine ME-111.4 mg/day [QR: 108.4; 115.3] (reference values (RV): 20-90 mg/ day); plasma norepinephrine $\mathrm{ME}$ - $14.3 \mathrm{pg} / \mathrm{mL}$ [QR: 13.1; 17.5] (RV: $3-$ $10 \mathrm{pg} / \mathrm{mL}$ ), plasma cortisol ME - $597.6 \mathrm{nmol} / \mathrm{L}$ [QR: 578.1; 608.3] (RV: $85-580 \mathrm{nmol} / \mathrm{L}$ ), ACTH ME - $15.8 \mathrm{pg} / \mathrm{ml}$ [QR: 13.9; 17.8] (RV: 9-52 pg/ $\mathrm{mL}$ ), glucose $\mathrm{ME}-5.2 \mathrm{mmol} / \mathrm{ml}$ [QR: $4.9 ; 5.5]$ (RV: 3.3-5.5 mmol/ml), insulin $\mathrm{ME}-8.2 \mu \mathrm{IU} / \mathrm{ml}$ [QR: 6.4; 12.5] (RV: $5-20 \mu \mathrm{IU} / \mathrm{ml}$ ).

\section{Conclusions}

FD in children is associated with imbalance in sympathoadrenal system as well as with such psychological disorders as anxiety, depression, sleep disorders, and cognitive dysfunction.

Key words: functional dyspepsia, stress, children, sympathoadrenal balance, psychological disorders.

\section{9}

\section{Paediatric Tropical Medicine}

Three cases of multidrug-resistant tuberculosis in children in Hasan Sadikin Hospital Bandung Indonesia: how we find the cases? Heda Melinda Nataprawira ${ }^{1}$, Sang Ayu Kompiyang Indriyani ${ }^{2}$, Ery Olivianto $^{3}$, Sri Sudarwati

${ }^{1}$ Department of Child Health, Faculty of Medicine Universitas Padjadjaran, Indonesia

${ }^{2}$ Department of Child Health, Faculty of Medicine Universitas Mataram, Indonesia

${ }^{3}$ Department of Child Health, Faculty of Medicine Universitas Brawijaya, Indonesia

\section{Background}

Increasing in adult MDR-TB cases may increase MDR-TB cases in children, because MDR-TB in children is mainly caused by transmission of drug-resistant strains, but the magnitude of this problem is less reported particularly in developing country. Even though until recently limited number of MDR-TB in children were identified globally, a recent systematic review of case series reported MDR-TB in children. In Indonesia, there is no data published and still challenging in diagnosis and treatment. Although we have MDR-TB children guideline adopted from WHO, it is difficult to implement in our setting because of lack laboratory facilities. Case Reports

Three cases of MDR-TB were diagnosed within the last year of the total 170 adult MDR-TB, which is our first clinical experience in diagnosing and treating MDR-TB in children. All three patients were female, one of them was under five years old. Two cases presented with chronic cough and one with scrofuloderma. One identified through contact investigation, and the others based on suspicion MDR-TB cases due to history of lost to follow up TB treatment and relapse of TB disease, respectively. However, the later two had no identified MDR-TB contact. Diagnoses of MDR-TB mainly based on GeneXpert MTB/RIF examination and DSTs. All patients are now still ongoing treatment with standard MDR-TB regimen, except one case of which treated with individual regimen due to sensory neural hearing loss identified before treatment start. 


\section{Conclusion}

MDR-TB in children should be identified both by case finding of MDRTB adult contacts and exploration history of lost to follow up and relapse of TB disease. It is noted that GeneXpert MTB/RIF is important in diagnosing MDR-TB in children.

51

\section{Primary Care}

Reflux Nephropathy Due To Asymptomatic Vesicoureteral Reflux Until The Age of 5

Elena Manoleva Nikolovska ${ }^{l}$

${ }^{T}$ Department of Pediatrics, PHI Polyclinic "Manolevi", Macedonia

\section{Backgrounds and Aims}

Vesicoureteral reflux (VUR) is an abnormal condition in which urine flows retrograde from the bladder into the ureters and kidneys. Vesicoureteral reflux can be primary or secondary. Girls,white children, infants and children up to age of 1 year appear to have a higher risk of vesicoureteral reflux.

Methods

A 5-year-old girl came on exame for routine laboratory tests of blood and urine. She was previosly healthy girl, with rare upper respiratory tract infections. Her mother was complaining on her child's poor appetite since her birth and weakness over the last period. There were no signs of urinary tract infection(UTI). Body weight and length were in the normal range of standard deviation.

The laboratory findings were: normal blood test, elevated serum Creactive protein.Urine analysis showed: 100 leukocytes, 4-5 erythrocytes, bacteria (+++). Urine culture: Escherichia coli 1000000/ml.

\section{Results}

The ultrasonography of the abdomen confirmed bilateral hydronephrosis grade 1 and smaller size of the right kidney. Left kidney was compensatory enlarged with an uneven surface.

99mTc-DMSA showed renal fixation LK/RK $=90 / 10 \%$. Right kidney showed reduction of the parenchyma and hypofixation of the whole kidney. Left kidney was enlarged,inhomogeneous, with hypofixation of the upper and lower half.

Intravenous urography excluded presence of kidney stones,confirmed bilateral hypotonia of ureters and hypoplasia of the right kidney. Cystourethrography during micturition showed VUR with dilatation of the left ureter distally of grade III.

Direct radionuclide cystography confirmed VUR lat. sin. of grade II-III and retention of the urine.

\section{Conclusions}

Early diagnosis of VUR is very important because of the prevention of episodes of acute pyelonephritis and kidney damage. Untreated UTI can lead to scarring, also known as reflux nephropathy, which is a permanent damage to the kidney tissue. Extensive scarring may lead to high blood pressure and can increase the risk of hypertension and renal failure in children and adults.

\section{1}

\section{Primary Care}

A Case Report of Partial Sigmoide Sinus and Transverse Left Sinus Thrombosis in a 15 Days Old Boy

Maria Luisa Arroba Basanta ${ }^{1}$, Begoña Manzarbeitia², Paloma Manzarbeitia ${ }^{3}$, Ana Cotes ${ }^{4}$

${ }^{1}$ Pediatrics, Complutense University of Madrid. Pozuelo Health Center., Spain

${ }^{2}$ School of Medicine, European University of Madrid, Spain

${ }^{3}$ School of Medicines, Complutense University of Madrid., Spain

${ }^{4}$ Pediatrics, Pozuelo Health Center, Spain

Cerebral venous sinus thrombosis in children is a rare complication and only few cases have been reported. Sinovenous thrombosis is probably less recognized and the symptoms and clinical course are highly variable. As in the present reported case, symptoms may be very non-specific. Availability of imaging techniques like magnetic resonance imaging (MRI) along with increasing awareness of condition should make it more frequently diagnosed.

A15 days old boy was refered to his pediatrician for rejection of mother lactation and discomfort. In the physical exploration he presents affectation of the general condition, light hipotonía and weak weeping. The rest of systemic examination was normal. He was sent to the hospital, where it enters for suggestive picture of sepsis/ meningitis.

On admission, initiates parenteral treatment with antibiotic and antiviral, previous withdrawal of LCR's sample for PCR of virus (negatives) and analytical. Within a few hours of the revenue he presents a pseudocomatose picture and a tonic crisis. EEG and transfontanelar ultrasound scan were normal.

Cerebral magnetic resonance showed partial sigmoide sinus and transverse left sinus thrombosis, without parenquima affectation. Sleep EEG and metabolic and coagulation study in blood and urine of 24 hours were normal.

After improving clinical, it realizes follow-up to the discharge in Neuropediatría and in a Center of Early Attention for precocious stimulation and neurological follow-up.

After two years of follow-up, the parents see him well. They came to Center of early attention, where it has been discharge at the age of 2 . He had not presented convulsive crises and psychomotor development is adapted to his age, without any neurological complaints.

195

\section{Primary Care}

PFAPA (Marshall Syndrome). Diagnosis Improvement after Implementation of Awareness Programm

Sorin Ioan Iurian ${ }^{1,2}$, Anca Vidrighin ${ }^{2,3}$

${ }^{T}$ Pediatric Clinic, Pediatric Hospital, Romania

${ }^{2}$ Pediatrics, Lucian Blaga University of Sibiu, Romania

${ }^{3}$ Pediatrics, Polisano Clinic, Romania

\section{Background}

PFAPA is a chronic auto-inflammatory disease including recurrent fever, aphthae, pharyngitis, adenitis. Steroids use from beginning of episode is able to stop it and no antibiotherapy is recommended.

\section{Objectives}

Authors analyzed the results after dissemination of information about PFAPA patients among general practitioners (GPs) in our county, in order not only to improve diagnosis but also to avoid unnecessary antibiotherapy.

\section{Methods}

Authors analyzed PFAPA children before and after implementation of a plan concerning improving PFAPA knowledge. Authors compared 2 PFAPA-groups: first group (patients diagnosed before programm implementation) and the second group (patients enrolled after programm application). Both groups were enrolled during 12 month-period and were evaluated according to same criteria: symptoms onset age, duration between episodes and period between disease onset and its confirmation. The programme was implemented in 2015 and comprised 4 meetings together with GPs. The patients were included in first group between january-december 2014. From january to december 2016 were enrolled patients belonging to second group. Patients up to 10 year-age fulfilling diagnosis criteria were analyzed.

Results

Seventy-nine patients were included in study: 18 patients (first group) and 61 PFAPA-children (second group). Mean age disease 
debut was 42.23 months (1st group) and 28.13 months for the 2nd. Duration from symptoms onset to diagnosis confirmation was 36.29 months (1st group) and 11.29 months for the second group, as a consequence of initiation of PFAPA awareness programm. Average period between fever attacks was 7.1 weeks (1st group) and 7.3 (2nd group) respectively.

Conclusions

1.Authors remarked a low suspicion index for PFAPA diagnosis among GPs before plan initiation; 2.PFAPA diagnosis war significantly improved after programm implementation (61 versus 18 cases); 3.PFAPA awareness programm was very useful and has to continue in order to avoid unjustified antibiotics, in the future, for PFAPA patients.

192

\section{Primary Care}

Child abuse in relation to social status and believes of the parents Nazih Ramadan ${ }^{1}$

${ }^{T}$ Forensic medicine and clinical toxicology, Faculty of medicine, Cairo university, Egypt

\section{Background}

Child abuse and neglect occur in epidemic numbers and around the world, resulting in major physical and mental health consequences for abused children in the present and future. Studying prevalence, causes, prevention and parental believes is very important for management of this big problem. The aim of this study is to highlight on the incidence and prevalence of child abuse in Egypt, the role of parental social and educational status in addition to their believes on this problem.

\section{Methodology}

The study was conducted on 507 randomly selected male and female parents seeking medical treatment in the pediatric hospital of faculty of medicine, Cairo University. They were asked after giving informed consent about their social data, believes about child abuse, incidence, frequency and how they abuse their children.

Results

$58.8 \%$ of the sample was male parents and $41.2 \%$ were females. $25.5 \%$ of them were illiterate while $21 \%$ were high school educated. Forty percent were smokers and $37 \%$ were non-workers. Thirty-four percent were physically abusing their children with $56 \%$ of them are frequently abusers, while $36 \%$ of sample emotionally abused their children. Eighteen percent of physical abuses resulted in an injury. Fifty-three percent of parents believe that physical and emotional punishment is important for child education, and $42 \%$ of parents thinks that physical punishment correct child attitude.

\section{Conclusion}

Child abuse is prevalent in Egypt with parents believe that abuse may be useful. Key words: Child, Abuse, Egypt, Parents

\section{5}

\section{Primary Care}

Gender Identity and Gender Dysphoria: Establishing Knowledge of Healthcare Professionals Working with Children Using Structured Interviews

Sophie Reeve ${ }^{l}$, Mary Hatton ${ }^{l}$, Stephanie Lai ${ }^{l}$, Torsten Hildebrandt ${ }^{1}$

${ }^{T}$ Department of Paediatrics, Abertawe Bro Morgannwg University

Health Board, UK

\section{Background}

Gender identity is an individuals' innate sense of being male, female, neither, or any combination of both. Gender dysphoria arises from incongruence between gender identity and birth-assigned gender, leading to intense distress and increased risk of psychiatric illness and suicide. The size of the transgender population is estimated to be between 0.4$1.3 \%$ of the general population. Despite increasing public awareness and rising referral rates, formal training in this area is lacking.

\section{Objective}

In recognition of this increasing problem, we conducted structured interviews among healthcare professionals at Abertawe Bro Morgannwg University Health Board to determine the baseline knowledge of issues surrounding gender identity.

\section{Method}

A 20-question, 10-minute structured interview was developed comprising three question types (open, multiple choice and true/false) structured around four themes:

- Definitions surrounding gender identity

- Child development and clinical presentation of gender dysphoria

- Referral pathways and timing of intervention

- Legal issues and their implications

A team of 3 peer-reviewed interviewers questioned 72 participants in February 2017, spanning various specialties (Paediatrics, Mental Health, Emergency Medicine, Surgery and Family medicine) and professions (Doctors, Nurses, Allied Health professionals).

\section{Results}

Many interviewees were unfamiliar with terminology surrounding gender identity, $60 \%$ being unaware of any services available to children on the NHS. The majority of Mental Health and Family Medicine professionals had a fundamental awareness of gender issues, legalities and referral pathways; however, paediatric specialists did not score significantly higher than other professionals.

\section{Conclusion}

General awareness of gender identity issues amongst professionals is limited, with our review suggesting that knowledge of paediatricians at our health board is no broader than that of professionals in other specialties. Given the rising referral rates of children with gender issues, there is a need for raising awareness of the subject amongst all professionals working with children.

\section{4}

\section{Primary Care}

Effect of Probiotic Microorganism Lactobacillus Reuteri on the Occurrence of Acute Otitis Media

Lilijana Besednjak-Kocijancic ${ }^{1}$

${ }^{T}$ Primary pediatric health centre, Zdravstveni dom Nova Gorica, Slovenia

\section{Background}

Human microflora and her changes in neonates are very important for the maturation of the immune system. Addition of probiotics to early child diet may improve immunity and prevent development of some diseases. Many reports suggest that certain probiotic strains have potent immunomodulatory activity in diverse disorders including acute otitis media (AOM). We aimed to determine whether breast feeding supplemented with probiotic Lactobacillus reuteri (LR) could reduce the risk of AOM in the first three years of life.

\section{Methods}

Three hundred eight healthy, maturely born infants divided in two groups (group A-194 infants exclusively breastfed for 4-6 months and group B-114 infants breastfed with addition of L. reuteri from fourth week of life for 12 weeks) were included in this prospective study. Every child was followed up by the same paediatrician until it was three years old. Epidemiological data about AOM at one and three years of age (frequency, episode duration and hospital admission rate) were analysed. Statistical analysis was performed with Epi 
Info 7 using chi-square analysis with Yates' correction. P-values less than 0.01 were considered significant.

\section{Results}

Percentage of children vaccinated with the 7-valent pneumococcal conjugate vaccine in groups A and B was nearly the same (group A: $24.2 \%$; group B: $24.6 \%$ ). In the first year of life had AOM $20.1 \%$ of children (group A: $26.2 \%$; group B: $9.6 \%$ ). Occurrence of AOM was significantly lower in group B (P0.001). Prevalence of AOM in first three years of life was $24.4 \%$ (group A: $30.4 \%$; group B $14 \%$ ), (P0.01). In the observed period frequency of episodes, duration of each episode and hospital admission rate was lover in the group B.

\section{Conclusions}

The study confirms that supplementation of breast feeding with probiotic L. reuteri reduce the risk of AOM. Addition of L. reuteri to child's diet is beneficial and has to be recommended.

\section{4}

\section{Primary Care}

Health Status of the Children Who Were Born after In Vitro Fertilization in Clinic "ISIDA"

Mariia Krepak ${ }^{l}$, Olena Platonova ${ }^{I}$, Andriy Vilenskiy ${ }^{I}$, Tetyana Veselova ${ }^{l}$, Olena Gayevskal

${ }^{1}$ Pediatric department, Clinic "ISIDA-IVF”, Ukraine

\section{Background}

Nowadays, in vitro fertilization (IVF) is a progressive type of contributed reproductive technology used for infertility treatment and gestational surrogacy. Objective

The aim was to examine the level of health condition in children 6 months of age who were born as a result of IVF in Clinic "ISIDA".

\section{Results}

We monitored the birth of 33 children after IVF for the 2015-2016 years. The control group consisted of 33 children who were born as a result of natural insemination from the same period. We observed that children after IVF have significantly older mothers $(36.5 \pm 2.9$ years old $)$ compared to the control group $(31.1 \pm 3.9$ years old). Furthermore, in $54.5 \%$ of cases children were born by caesarean section, compared to control group $33.3 \%$. However, among the newborns after IVF males were dominated - $60.6 \%$, compared to control group - $51.5 \%$. In addition, there were 6 sets of twins after IVF, and one set of twins in control, $\mathrm{p}$ 0.05. Also, we described body weight and height of newborns, where indicators of children in the IVF group were lower compared to control group ( $2880.6 \pm 720.0 \mathrm{~g}, 49.9 \pm 4.5 \mathrm{~cm}$ in tested group, and $3265 \pm 492.7 \mathrm{~g}, 51.8 \pm 2.6 \mathrm{~cm}$ in control group, p0.05). We observed that children after IVF gained weight worse than control group during first six months of life. Among children after IVF $36.4 \%$ were bottle-fed, compared to control group $12.2 \%$, but no increased risk of atopic dermatitis and functional gastrointestinal disorders were found.

\section{Conclusion}

Consequently, our results are significant for the various parametres of further monitoring health status in children who were born after IVF in Clinic "ISIDA".

394

\section{Primary Care}

Invasive pneumococcal disease in a primary paediatric practice in Croatia

Helena Zivic ${ }^{1}$

${ }^{T}$ Paediatrics, Health facility Zagreb Center, Croatia

\section{Background}

Invasive pneumococcal disease (IPD) is a major health problem in Croatia. Most cases are caused by less than 10 pneumococcal serotypes and there are opportunities for prevention in the form of conjugated vaccines.

\section{Objective}

Aim was to analyze cases of IPD in our primary paediatric practice. Methods

We analyzed epidemiological characteristics of 18 cases of IPD treated in the period from January 2014 - January 2017. Descriptive statistical analysis was used.

Results

From a total of 18 patients, 11 were male and 7 female. The mean age of the patients was 16 months, median 14 months, age range from 9 months to 29 months. Nine patients had a diagnosis of bacteriemia, 8 had bacteriemic pneumonia, and 1 had bacterial meningitis. Antimicrobial treatment applied was ceftriaxone parenterally and later amoxicillin orally. Clinical improvement was observed in all patients. Basic immunological findings were normal in all children. The one patient with bacterial meningitis had transient neutropenia during infancy. Most of the cases occurred during winter months, only the case of bacterial meningitis during early spring. Most of the patients attended kindergarten and weren't vaccinated with PCV13 vaccine prior the disease. Parents of all of these patients decided to vaccinate their child with PCV13 vaccine after the recovery.

Conclusion

Most cases of IPD occur in otherwise healthy children and could be prevented by existing conjugate vaccines (PCV10 and PCV13 vaccine).

\section{1}

\section{Primary Care}

Relationship of Some Easily Assessed Factors with Suicidality in Children and Adolescents

Amy Manning $^{I}$, Kim Bennett $^{I}$, Shelley Hitzel ${ }^{1}$, Catherine Dulmus ${ }^{1}$, Thomas Nochajski ${ }^{l}$

${ }^{I}$ Social Work, University at Buffalo, The State University of New York, USA

\section{Background}

Mental health needs often go unmet in children and adolescents. Suicide is in the top three leading causes of death in the United States for young people ages 10-34 (CDC, 2015). Although, the number of completed suicides remains relatively low, the number of youth reporting suicidality continues to increase. Youth experiencing suicidality may be present in our physicians' offices more often than the numbers of completed suicides would lead us to believe.

Objective

Identify suicidality related factors that can be assessed in a clinical setting. Methods

The study used responses of 5918 high school students from one mid-size urban school district on the Youth Risk Behavior Survey (YRBS), an online survey, to assess relationships between specific items and suicide attempts. Among the items included were some from the Adverse Childhood Experiences (ACE) questionnaire. Logistic regressions were performed the relationships of 10 of the items from the YRBS with attempted suicide.

\section{Results}

In the 12 months prior to the survey, $16 \%$ of students reported they had thought about suicide and $7.4 \%$ reported attempting suicide. Results showed that females were more likely than males to attempt a suicide. The logistic regressions showed feelings of hopelessness, dating violence, and being cyber bullied were the strongest predictors of a subsequent suicide attempt. All five of the ACE items also showed strong relationships with a suicide attempt. (see Table 1).

\section{Conclusion}

Youth who experience violence, assault and abuse are likely to be seen in clinical settings by Pediatricians. Identifying factors related to suicidality among patients and providing screening, assessment, intervention and support may assist in filling the unmet mental health needs of our youth. Directing conversation throught these topics during standard practice visits with adolescents is unobtrusive and may serve to both identify suicidality and improve $\mathrm{Dr} /$ patient relationships. The intersection of physical and mental health remain an important avenue for intervention. 
Table 1 Logistic Regression for Items with Attempted Suicide

\begin{tabular}{|c|c|c|c|c|c|c|c|c|}
\hline \multirow[t]{2}{*}{ Variable } & \multirow[t]{2}{*}{ B } & \multirow[t]{2}{*}{ se } & \multirow[t]{2}{*}{ Wald } & \multirow[t]{2}{*}{ df } & \multirow[t]{2}{*}{ Sig. } & \multirow[t]{2}{*}{$\operatorname{Exp}(B)$} & \multicolumn{2}{|c|}{$95 \%$ a for (Exp(B) } \\
\hline & & & & & & & Lower & Upper \\
\hline Hopeless & 2.692 & .123 & 475.270 & 1 & .0001 & 14.761 & 11.588 & 18.803 \\
\hline Being Bullied & 1.894 & .109 & 300.967 & 1 & .0001 & 6.644 & 5.364 & 8.229 \\
\hline $\begin{array}{l}\text { Being Cyber } \\
\text { Bullied }\end{array}$ & 2.198 & .114 & 373.781 & 1 & .0001 & 9.004 & 7.206 & 11.251 \\
\hline Emotional Abuse & 1.531 & .117 & 171.464 & 1 & .0001 & 4.625 & 3.678 & 5.817 \\
\hline Physical Abuse & 1.604 & .116 & 189.546 & 1 & .0001 & 4.972 & 3.957 & 6.247 \\
\hline Sexual Abuse & 2.053 & .129 & 253.319 & 1 & .0001 & 7.793 & 6.052 & 10.035 \\
\hline $\begin{array}{l}\text { Exposure to } \\
\text { Alcohol/Drugs in } \\
\text { Home }\end{array}$ & 1.186 & .110 & 117.021 & 1 & .0001 & 3.275 & 2.642 & 4.061 \\
\hline $\begin{array}{l}\text { Exposure to } \\
\text { Mental } \\
\text { IIIness/suicide }\end{array}$ & 1.719 & .112 & 234.125 & 1 & .0001 & 5.579 & 4.477 & 6.954 \\
\hline $\begin{array}{l}\text { Witness } \\
\text { Violence in the } \\
\text { Community }\end{array}$ & 1.154 & .111 & 108.866 & 1 & .0001 & 3.170 & 2.552 & 3.937 \\
\hline Dating Violence & 2.457 & .132 & 345.597 & 1 & .0001 & 11.667 & 9.005 & 15.117 \\
\hline
\end{tabular}

614

\section{Primary Care}

Age and Outcome of Inpatients Evaluated for Possible Physical Abuse at a Tertiary Children's Hospital in Greece

Alexandra Soldatou ${ }^{1}$, Bilio Paouri ${ }^{1}$, Anna Hountala ${ }^{2}$, Eleni Koutrouveli ${ }^{2}$, Eleni Plevriti $^{3}$, Tatiana Kyriakidou ${ }^{4}$, Afroditi Stathi ${ }^{5}$, Maria Tsolia ${ }^{1}$, Resmiye Oral ${ }^{6}$, John M. Leventhal

${ }^{1}$ 2nd Department of Pediatrics, National and Kapodistrian University of Athens, Greece

${ }^{2}$ Department of Radiology, "P. \& A. Kyriakou” Children's Hospital of Athens, Greece

${ }^{3}$ Department of Social Work, "P. \& A. Kyriakou” Children's Hospital of Athens, Greece

${ }^{4}$ Department of Child Psychiatry, "P. \& A. Kyriakou” Children's Hospital of Athens, Greece

${ }^{5}$ ELIZA, Society for the Prevention of Cruelty to Children, Greece

${ }^{6}$ Department of Pediatrics, University of Iowa, USA

${ }^{7}$ Department of Pediatrics, Yale School of Medicine, USA

\section{Background}

There is no child abuse registry in Greece.

\section{Objective}

To determine the age and outcome of inpatients evaluated for possible physical abuse at a tertiary children's hospital in Greece.

Methods

Children aged $0-16$ years with a report of physical abuse to the juvenile attorney or with injuries and social concerns hospitalized from January 2014-December 2015 were retrospectively identified from the handwritten archives of the hospital departments of Social Work and Child Psychiatry. Based on paper records and imaging studies, two pediatricians and two radiologists reached a consensus rating on the likelihood of abuse on each case using a 7-point Clinical Abuse Scale with three levels of confidence for abuse (definite, likely, and questionable), an indeterminate middle rating and 3 levels of confidence for accident (definite, likely, and questionable). Place of discharge was noted as protected (kinship or institution) or unprotected (home or unknown).

\section{Results}

Of the 39 patients identified:

- The mean age was 6.8 years ( 1.5 months - 16 years).

- Reports to the juvenile attorney were made by the community in 26 and by hospital physicians in 7 patients.

- 6 additional patients were reviewed due to injuries and social concerns.

- The researchers categorized 22 as definite abuse, 2 as likely abuse, 4 as questionable abuse, 3 as likely accident and 8 as indeterminate cause.

- All 6 infants were categorized as abuse: 3 definite, 1 likely and 2 questionable.

- Of the 28 patients categorized as abuse:

- The mean age was 7.7 years (1.5 months - 16 years).

- 2 died (charges were pressed against the caregivers of one deceased child).

- 10 were discharged to an unprotected, and 16 to a protected environment.

\section{Conclusion}

This review of 39 inpatients evaluated for possible physical abuse at a children's hospital in Greece suggests suboptimal detection and management of child abuse.

664

\section{Primary Care}

Missed Opportunities for the Detection of Physical Abuse and Neglect among Patients Hospitalized with Burns at a Tertiary Children's Hospital in Greece

Alexandra Soldatou ${ }^{1}$, Bilio Paouri ${ }^{1}$, Afroditi Stathi ${ }^{2}$, Chrisanthi Nega ${ }^{3}$, Maria Tsolia ${ }^{l}$, Resmiye Oral ${ }^{4}$, John M. Leventhal ${ }^{5}$

${ }^{I}$ 2nd Department of Pediatrics, National and Kapodistrian University of Athens, Greece

${ }^{2}$ ELIZA, Society for the Prevention of Cruelty to Children, Greece

${ }^{3}$ DEREE, The American College Greece, Greece

${ }^{4}$ Department of Pediatrics, University of Iowa, USA

${ }^{5}$ Department of Pediatrics, Yale School of Medicine, USA

\section{Background}

Existing data indicate that child physical abuse and neglect may be underrecognized and under-reported in Greece.

\section{Objective}

To determine possible missed opportunities in detecting physical abuse and neglect among children hospitalized for burns at a tertiary children's hospital in Greece.

\section{Methods}

Children aged 0 - 6 years hospitalized from January 2014-December 2015 with a discharge diagnosis of burn were retrospectively identified from the hospital electronic discharge database. Based on paper records, two pediatricians reached a consensus rating on the likelihood of abuse on each case using a 7-point Clinical Abuse Scale with three levels of confidence for abuse (definite, likely, and questionable), an indeterminate middle rating and 3 levels of confidence for accident (definite, likely, and questionable). One subcategory in the categories of likely and questionable accident involved neglect. Any documentation of indicators of abuse or neglect in the records was noted. Results

Of the 67 patients identified:

- The mean age was 1.8 years ( 3.5 months -5.1 years).

- The researchers categorized 1 as definite abuse, 11 as questionable abuse, 13 as questionable accident, 33 as likely accident, and 9 as indeterminate cause. - 23 out of 46 cases categorized as questionable and likely accident involved neglect.

- There was no documentation of indicators of abuse or neglect in the records reviewed.

- Of the 12 patients categorized as abuse:

- The mean age was 1.9 years ( 5 months -4.5 years).

- The single case categorized as definite abuse and 6 out of 11 cases categorized as questionable abuse were infants. 


\section{Conclusion}

This review of 67 children hospitalized at a children's hospital in Greece with burns suggests missed opportunities for the detection of child abuse and neglect.

681

\section{Primary Care}

The relationship between the mode of arrival at pediatric emergency department and severity in age categories in Japan Yoshihiko Morikawa ${ }^{1}$, Shogo Kato ${ }^{2}$, Yusuke Hagiwara ${ }^{3}$, Naohisa Yahagi ${ }^{4}$ ${ }^{1}$ Clinical Research Support Center, Tokyo Metropolitan Children's Medical Center, Japan

${ }^{2}$ Division of Data Science for Clinical Research, National Center for Child Health and Development, Japan

${ }^{3}$ Tokyo Metropolitan Children's Medical Center, Department of Pediatric Emergency and Critical Care Medicine, Japan

${ }^{4}$ Graduate School of Media and Governance, Keio University, Japan

\section{Background}

Diseases, severity, or parents' anxiety differ according to the age in childhood. Although the age may influence the mode of arrival at an emergency department (ED) and disposition, the hospitalization rate by the mode of arrival in each age category is unclear yet.

\section{Purpose}

To clarify the relationship between the mode of arrival at ED and the admission rate in each age category in Japan.

Methods

A retrospective cohort study was performed for patients under age of 18 years who visited ED at two pediatric tertiary hospitals in Japan from August 2014 through September 2016. These patients were classified into 6 age categories: group 1 as birth to 28 days; group 2 as 29 days to 12 months; group3 as 13 months to 2 years; group 4 as 3 to 5 years; group 5 as 6 to 11 years; and group 6 as 12 to 18 years. The mode of arrival at ED and rate of admission to wards were examined.

\section{Results}

A total of 140,412 cases were eligible, with group $10.7 \%$, group $214.0 \%$, group $329.9 \%$, group $418.8 \%$, group $528.9 \%$, and group $67.7 \%$, respectively. Patients who arrived by ambulance for each age group occupied $17.8 \%, 8.6 \%, 10.7 \%, 8.9 \%, 8.7 \%$, and $11.5 \%$, respectively. The admission rate in patients who arrived by walk-in and ambulance for each age group was $8.6 \%$ vs. $51.2 \%$ (OR 11.2, $95 \%$ CI $7.6-16.5$ ), $5.1 \%$ vs. $23.8 \%$ (OR 5.8, $95 \%$ CI $5.1-6.7$ ), $3.4 \%$ vs. $8.8 \%$ (OR 2.7, $95 \%$ CI $2.4-3.2$ ), $2.8 \%$ vs. $8.7 \%$ (OR 3.3, $95 \%$ CI $3.0-3.7$ ), $2.9 \%$ vs. $10.1 \%$ (OR $3.7,95 \%$ CI $3.2-$ 4.3 ), and $3.6 \%$ vs. $9.4 \%$ (OR 2.8, $95 \%$ CI $2.2-3.4$ ), respectively.

Conclusion

Patients who arrived by ambulance were more frequently in group 1 and the admission rate was higher in group 1 and 2 in Japan.

682

\section{Primary Care}

Physical abuse potential in mothers of children and adolescents with an Intellectual Disabilities

Hatice Yildirim Sari ${ }^{1}$, Esra Ardahan ${ }^{I}$, Beste Oztornaci ${ }^{l}$, Alev Girli ${ }^{3}$, Bumin Nuri Dundar ${ }^{2}$

${ }^{1}$ Pediatric Nursing Department, İzmir Katip Celebi University Faculty of Health Science, Turkey

${ }^{2}$ Pediatric Endocrinology Department, İzmir Katip Celebi University Medical Faculty, Turkey

${ }^{3}$ Special Education Department, Dokuz Eylul University Education Faculty, Turkey

\section{Objective}

The aim of this study was to determine physical abuse potential in mothers of children and adolescents with intellectual disabilities.

\section{Methods}

This study was designed as a descriptive one. Data were collected from 128 parents with the survey method using the Family Information Form, Child Abuse Potential Inventory (CAPI).

\section{Results}

The mean score the participating mothers obtained from the abuse subscale of the CAPI was $179.97 \pm 89.85$ ( $\min : 27$, $\max : 404)$. Of the participants, $37.3 \%(n=47)$ were found to pose risks in terms of perpetrating abuse. CAPI scores of the participating mothers were determined to be associated with children's disability levels, children's anger and irritability behaviors, the mother's and father's education level, and the mother's employment status and the family's economic status.

\section{Conclusion}

In this present study, approximately $40 \%$ of the mothers of the children with intellectual disabilities posed risks in terms of perpetrating abuse, and low education and economic levels increased the abuse potential.

\section{3}

\section{Primary Care}

Assessment of the Prevalence of Obesity, Stunting and Hypertension among Primary School Children

Medine Yllmaz, ${ }^{1}$ Betul Aktaş $^{3}$, Feyza Dereli ${ }^{1}$, Hatice Yildirim Sari ${ }^{2}$, Gamze Agartioglu Kundakcl ${ }^{1}$, Zeliha Tiraki ${ }^{4}$

${ }^{1}$ Public Health Nursing Department, Izmir Katip Celebi University Faculty of Health Science, Turkey

${ }^{2}$ Pediatric Nursing Department, İzmir Katip Celebi University Faculty of Health Science, Turkey

${ }^{3}$ Pediatric Nursing Department, SANKO University Faculty of Health Science, Turkey

${ }^{4}$ Nursing Department, Ege University Medical Faculty Hospital, Turkey

\section{Objectives}

The study's aim was to investigate the prevalence of obesity, stunting, and hypertension problems among 5-14-year-old students studying at three primary schools.

\section{Methods}

The sample of this cross-sectional study comprised 2930 primary school children (1st -8 th grade). The participants' heights, weights and blood pressures were measured.

\section{Results}

Of the participants, $17.4 \%$ were overweight, $22.8 \%$ were obese, $1.1 \%$ stunted, and $5.9 \%$ were short. In the eight-year age group, the percentage of overweight boy students was higher than that of the overweight girl students. The proportions of the students with stage I and stage II systolic hypertension were $6.8 \%$ and $3.2 \%$ respectively. While $1.1 \%$ of the students had stage I diastolic hypertension, $5.5 \%$ had stage II diastolic hypertension.

\section{Conclusions}

The prevalence of stunting, overweight and Stage I and II hypertension among the children aged 6-14 was high. Thus, it is important to early identify such problems among children and to take precautions by carrying out routine screenings in schools.

\section{1}

\section{Primary Care}

The relationship between chief complaint and hospitalization rate in age categories in pediatric emergency department in Japan Yoshihiko Morikawa ${ }^{1}$, Shogo Kato $^{3}$, Yusuke Hagiwara ${ }^{2}$, Naohisa Yahagi $^{4}$ ${ }^{T}$ Clinical Research Support Center, Tokyo Metropolitan Children's Medical Center, Japan

${ }^{2}$ Department of Pediatric Emergency and Critical Care Medicine,, Tokyo Metropolitan Children's Medical Center, Japan

${ }^{3}$ National Center for Child Health and Development, Division of Data Science for Clinical Research, Japan

${ }^{4}$ Graduate School of Media and Governance, Keio University, Japan 


\section{Background}

Medical interview information contributes to diagnosis. Differential diagnosis and severity is different according to the age in childhood. However, the relationship between age, severity and admission rate in children with the chief complaint $(\mathrm{CC})$ related respiratory symptom is unclear yet.

\section{Purpose}

To clarify the relationship between CC related respiratory symptom and hospitalization rate in each age categories at emergency department (ED) in Japan.

\section{Methods}

A retrospective cohort study was performed for patients under age of 18 years who visited ED at two pediatric tertiary hospitals in Japan from August 2014 through September 2016. These patients were classified into 6 age categories: group1 as birth to 28 days; group2 as 29 days to 12 months; group 3 as 13 moths to 2 years; group 4 as 3 to 5 years; group 5 as 6 to 11 years; and group 6 as 12 to 18 years. CC, triage category using Canadian Triage Acuity and Scale (CTAS), and rate of admission to wards were evaluated by age group.

\section{Results}

A total of 19,748 case were eligible. In all ages, coughing and nasal discharge were the most frequent $\mathrm{CC}$. The next most frequent $\mathrm{CC}$ was infant apnea in group 1 , wheezing in group 2 to 5 , and allergic reactions in group 6 . The frequent CCs in patients who had admitted each group were as below: stridor, wheezing, and infant apnea in group 1; infant apnea, respiratory arrest, and shortness of breath $(\mathrm{SOB})$ in group 2; hemoptysis, $\mathrm{SOB}$, and wheezing in group 3; respiratory arrest, $\mathrm{SOB}$, and wheezing in group 4; $\mathrm{SOB}$, wheezing, and stridor in group 5; and respiratory arrest, foreign body, and wheezing in group 6 .

\section{Conclusion}

The relationship between $\mathrm{CC}$ and admission rate differed according to the age categories. To evaluate patients' condition, medical interview considering patients' age must be important.

\section{4}

\section{Primary Care}

Impact of Evidence-Based Behavior Change Communication on Infant and Young Child Feeding (IYCF) Practices in Tribal Gujarat, India

Kandarp Talati ${ }^{1}$, Ajay Phatak ${ }^{2}$, Bankim Gandhi ${ }^{3}$, Krisha Talati ${ }^{1}$, Dipen Patel $^{4}$, Karamchand Patil ${ }^{2}$, Mayur Shinde ${ }^{2}$, Somashekhar Nimbalkar ${ }^{2,4}$ ${ }^{1}$ Department of Interdisciplinary Research, Foundation for Diffusion of Innovations, India

${ }^{2}$ Central Research Services, Charutar Arogya Mandal, India

${ }^{3}$ Department of Tribal Development, Foundation for Diffusion of Innovations, India

${ }^{4}$ Department of Pediatrics, Pramukhswami Medical College, India

\section{Background}

Poor IYCF-practices increase the risk of malnutrition and poor developmental outcomes. Low literacy, poor socio-economic conditions, and missed opportunities for counselling in hard-to-reach, interior tribal villages may adversely influence IYCF-practices.

\section{Objective}

To assess impact of behaviour change communication(BCC) on IYCFpractices in 6-12 $\mathrm{M}$ infants in tribal Gujarat, India

\section{Design/Methods}

Two blocks were randomly assigned to control $(\mathrm{CC} ; N=541)$ and intervention(IC; $N=474)$ clusters. Pregnant women in their third trimester (Cohort-1; $N=610$ ) and mothers of $0-3 \mathrm{M}$ infants (Cohort-2; $N=405$ ) were recruited in both clusters at baseline $(N=1015)$ during Apr-Jul'16. Enrollee in the intervention group received interpersonal BCC for ageappropriate IYCF-practices (including discouragement of traditional, harmful feeding practices) at baseline, mid-line(Aug-Dec'16), and recipe demonstration and group counselling(Dec'16-Jan'17). End-line assessment (Jan-Mar'17) assessed the impact of BCC on maternal awareness and IYCF-practices.

\section{Results}

At baseline, maternal profile and awareness about IYCF-practices were similar in IC and CC. Lost-to-follow-up much higher than anticipated at end-line in CC and IC (228; $48 \%$ vs. $299 ; 55 \%)$ mainly attributed to out-migration. Being male $(129 ; 48.86 \%$ vs. $89 ; 37.71 \%)$ and first child $(70 ; 52.24 \%$ vs. $148 ; 40.44 \%)$ were significantly associated (p0.02) with minimum meal frequency (MMF) for 6-8 M and 9-12 M infants. Minimum dietary diversity (MDD), i.e. infants receiving foods from four or more food groups, were similar $(18 ; 7.29 \%$ vs. $16 ; 6.32 \%)$ in IC and CC. Among those who failed to achieve MDD, 35;14.17 \% and 29;11.46\% in IC and CC received foods from three food groups, whereas $90 ; 36.44 \%$ and $86 ; 33.99 \%$ did not receive any complementary feeding in past 24 hours.

\section{Conclusion(s)}

MDD practice improved during end-line compared to mid-line during which none of the infants in cohort- 2 in IC and $\mathrm{CC}$ received meals from 4 or more food groups. However, both MMF and MMD remained suboptimal over the course of the intervention assessment. While socioeconomic status, food insecurity and migration could influence CF practices, further research is required to identify context-specific barriers and locally adaptable solutions to curb malnutrition in tribal population.

\begin{tabular}{|r|c|c|c|}
\hline & Base-line & Mid-line & Bnd-line \\
\hline Cohort I & Pregnant, third-trimester & $0-3$ month & $>5$ month \\
\hline Cohort II & $0-3$ month & $>5$ month & $>7$ month \\
\cline { 2 - 4 } & \multicolumn{2}{|c|}{ Table 1: Approximate age-group at different stages of assessment } \\
\hline
\end{tabular}

\section{6}

\section{Primary Care}

\section{The Story of a Hiding Disease}

Peter Altorjai ${ }^{1}$, Margit Lörincz ${ }^{2}$, Krisztián Kállay ${ }^{3}$

${ }^{T}$ Tóth Ilona Healthcare Center, Association of Hungarian Primary Care Paediatricians, Hungary

${ }^{2}$ Gastroenterology and nephrology Department, Heim Pál Children's Hospital, Hungary

${ }^{3}$ Pediatric haematology and stem call department, Egyesitett Szent István és Szent László Kórház, Hungary

\section{Background}

Paediatric malignancies are rare diseases, however their incidence show a mild increase worldwide. Certain types of tumours may show more typical symptoms, while others can imitate numerous less pathologic conditions affecting organs of the corresponding anatomical locations.

Due to the growing incidence and an often non-specific clinical course it is particularly important to keep track of these not univocal signs besides taking careful medical history, and performing thorough physical examination. Objective

The presentation will discuss the case of a currently 7-year-old boy, whose persistent, non-specific symptoms' background was failed to be identified despite of multiple examinations partly initiated by his parents. Although the infant was examined because of diverse neurodevelopmental and gastroenterological complaints as early as from 2 months of age by neurologists, radiologists and a gastroenterologist, he was eventually diagnosed with stage IV neuroblastoma having found metastatic lymph nodes at the age of 1 year old.

Chemotherapies of two consecutively applied protocols did not result in decrease of the size of the inoperable tumour, however, full regression was achieved at metastasis level. According to the decision of the Tumour Board, the child has not been given any treatment since the tumour has been showing no activity for several years.

\section{Conclusion}

Approximately $1 / 4$ of tumours occurring under the age of 14 years is of central nervous system origin, amongst these neuroblastoma is highly typical in infants and toddlers. Neoplasms appearing at various ages 
manifest diverse symptoms due to age characteristics, and their prognosis is significantly different.

Although in this case there were more, non-specific, progressive symptoms and complaints due to the growth of the active tumour, their holistic interpretation did not happen on any level of healthcare.

Hopefully through presenting this case and discussing international clinical guidelines, a hidden disease like this will be earlier and easier diagnosed.

\section{4}

\section{Respiratory}

Alpha-1 Antitrypsin and Wheezing in Early Childhood: Pilot Study Tomas Alasevicius ${ }^{1}$, Joanna Chorostowska-Wynimko ${ }^{2}$, Algirdas Valiulis ${ }^{3}$, Adriana Rozy ${ }^{2}$, Vaida Taminskiene ${ }^{4}$, Arunas Valiulis ${ }^{1,4}$

${ }^{1}$ Clinic of Children's Diseases, Vilnius University, Lithuania

${ }^{2}$ Department of Genetics and Clinical Immunology, National Institute of Tuberculosis and Lung Diseases, Poland

${ }^{3}$ Department of Rehabilitation, Physical and Sports Medicine, Vilnius University, Lithuania

${ }^{4}$ Public Health Institute, Vilnius University, Lithuania

\section{Background}

Alpha-1 antitrypsin (AAT) deficiency is an inherited disorder in which the plasma levels are diminished and affected people carry an increased risk of early onset of chronic obstructive pulmonary disease (COPD). Wheezing in early childhood is associated with acute respiratory infections and certain wheezing phenotypes have clinical features similar to COPD (Kerkhof M, 2014).

Objective and methods

Preschool children who present with an acute episode of wheezing are enrolled, clinically evaluated and blood samples are taken. AAT evaluation includes plasma level assessement by nephelometry, phenotyping by isoelectric focusing and genotyping by real-time polymerase chain reaction. In case of uncertainty, SERPINA1 gene sequencing is performed. Primary endpoint is the association between AAT variants and clinical wheezing phenotypes as defined by the European Respiratory Society (Brand P, 2014). Results

Twenty-eight wheezing children (median age 19.1 months; 13 female) were enrolled in a prospective pilot study. The majority of children (85.8 \%) had wheezing episodes due to viral respiratory infection. The mean level of AAT was $162.4 \mathrm{mg} / \mathrm{dl}$ (range $87-215$ ). Two cases (7.1\%) of PiM1M1, one (3.5\%) PiMS and one (3.5\%) PiMZ were detected. Normal genotype (PiMM) was found in others. The 3-year-old child with PiMS genotype had a wheezing episode with more severe respiratory distress although his AAT levels were normal (140 mg/dl). The 2-year-old child with a PiMZ genotype (AAT level $87 \mathrm{mg} / \mathrm{dl}$ ) experienced virus-induced wheezing with mild respiratory impairment. Both children had no history of atopy nor second-hand smoke exposure. We found no association between AAT concentration and respiratory distress severity nor with the time of first wheezing episode.

\section{Conclusions}

The pilot study has identified three cases of viral induced wheezing with uncommon genetic variants of AAT and normal AAT levels. Further research is needed to better understand the effects of AAT genotype not related to its concentration.

276

\section{Respiratory}

Projection of children diseases into adulthood: if wheezing stop it means no asthma?

Tomas Alasevicius ${ }^{1}$, Arunas Valiulis ${ }^{1,2}$, Vaida Taminskiene ${ }^{2}$, Algirdas Valiulis ${ }^{3}$

${ }^{1}$ Clinic of Children's Diseases, Vilnius University, Lithuania

${ }^{2}$ Institute of Public Health, Vilnius Unviersity, Lithuania

${ }^{3}$ Department of Rehabilitation, Physical and Sports Medicine, Vilnius University, Lithuania

Wheezing is a major medical and social problem affecting up to $50 \%$ of children under the age of 6 years. Asthma diagnosis in early childhood is challenging not only due to symptoms recognition, but current clinical tactics of paediatricians to postpone asthma diagnosis in children of first 3 years of age as well. The fundamentals to postpone diagnosis is based on disappearance of wheezing symptoms in majority of children before school age and the attempt to explain course of the disease using adult asthma model. ERS proposed a definition of wheezing phenotypes based on the patterns of symptom presentation, namely the episodic viral wheeze (EVW) and multiple-trigger wheeze (MTW). The most recent studies found only a small fraction of both phenotypes to be stable over a 2-year period. Although the odds for developing asthma were greater in those with stable MTW some children with EVW also developed "chronic" asthma. On the other hand, transient early wheeze is associated with chronic obstructive pulmonary disease (COPD) genes. Longitudinal cohort studies allowed distinguishing up to 7 phenotypes of wheezing, but this complex phenotyping is unused in daily practice. Current data need to be unified, simplified, and a prognosis based phenotyping of wheezing syndrome is to be developed.

Two major clinical asthma phenotypes are proposed: the 1st or "children's phenotype" that is characterized by early onset and most likely transient course (symptom-free by school age, but high risk for COPD in adulthood), not associated with atopy nor familial history of asthma, and the 2nd or "adults' phenotype" which is characterized by later onset wheezing usually beyond the age of 3 years, most likely persistent symptoms into adult age, and associated with atopy and/or asthma in close relatives. Further research is needed for better translation of wheezing phenotypes into clinical practice.

302

Respiratory

Characteristics of Pulmonary Tuberculosis Among Children With Congenital Hypothyroidism: Situation in West Java, Indonesia Citra Cesilia ${ }^{1}$, Faisal $B^{1}$, Julistio TB Djais ${ }^{1}$, Heda Melinda Nataprawira ${ }^{1}$ ${ }^{T}$ Department of Child Health, Universitas Padjajaran, Indonesia

\section{Background}

The prevalence of pulmonary tuberculosis (PTB) in children was high in Indonesia. PTB can exist with many other diseases, such as congenital hypothyroidism $(\mathrm{CH})$ because they are susceptible to various microorganisms. $\mathrm{CH}$ patients was hospitalized commonly due to lung infection. Until now, there is no data and previous studies of the characteristics of PTB among $\mathrm{CH}$ children.

\section{Objective}

To identify the characteristics of PTB in $\mathrm{CH}$ children and to determine its prevalence.

\section{Methods}

A cross-sectional study conducted from January 2012 to December 2016 involving $205 \mathrm{CH}$ children who were registered in Hasan Sadikin General Hospital Bandung. We identified prevalence of PTB, age, gender, residency, nutritional status, hypothyroid screening status at birth, hospitalization frequencies, and length of stay of hospitalization.

Results

Seventeen $(8.3 \%)$ of $205 \mathrm{CH}$ patients was diagnosed of having PTB. The mean age of them was $2.5 \pm 2.9$ months. Most of PTB patients were infants $(52.9 \%)$ and male $(82.4 \%)$ were preponderance. There are mostly $(64.7 \%)$ living outside of Bandung city with low educated and socioeconomic condition. We found almost all of the patient was malnourished (76.4 \%). Newborn hypothyroidism screening was not done in all $\mathrm{CH}$ patients. Most of them was diagnosed PTB when hospitalized for second admission (47\%) or more (41\%). The hospitalization length of stay amongst patients with PTB was between 1-2 weeks $(58.8 \%)$.

\section{Conclusions}

PTB prevalence in $\mathrm{CH}$ patients is low. Most of them was diagnosed PTB when hospitalized for second admission or more because of lung infection and PTB may extend length of stay. All of them is never done newborn hypothyroid screening.

Keywords: Pulmonary tuberculosis, congenital hypothyroidism, malnutrition 
501

\section{Respiratory}

Procalcitonin importance in monitoring and prognosis of lower respiratory tract infections in children

Horga Adeline Larisa ${ }^{1}$

${ }^{T}$ PhD Programs, Lucian Blaga University of Sibiu, Romania

\section{Introduction}

Lower respiratory tract infections in children is the most frequent cause of hospitalization in developed countries and the second leading cause of death in developing countries, prompting intense study of this pathology. The serum level of Procalcitonin it is increased in bacterial infections, increase rapidly in 3 to 6 hours after the onset of infection, which helps to monitor the evolution and in the same time the severity of the infection. The level of procalcitonin concentration reflects the systemic response to bacterial infection and its severity.

\section{Methods}

The study involved 105 children who were hospitalized in Pediatric Clinic Hospital of Sibiu between January 2013 and January 2016. Children hospitalized with lower respiratory tract infections, who presented at the admission in the hospital: malaise, fever, respiratory functional signs, the biological samples have confirmed us the bacterial infection and chest $\mathrm{x}$-ray amended. We determined procalcitonin at hospital admission and during antibiotherapy in order to monitor the clinical evolution.

\section{Results}

The most of the hospitalized children were male $56,19 \%$, from urban areas $59,04 \%$. All patients at admission in the hospital have had the value of procalcitonin increased, the value higher than $0.5 \mathrm{ng} / \mathrm{mL}$, but after 3 to 5 days of antibiotics the value of procalcitonin has decreased. $60.95 \%$ of patients received only one type of antibiotic, and 39, $04 \%$ have had associated two antibiotics. Most cases had favorable prognosis $(74.28 \%)$ with negative procalcitonin after the treatment, and $25,71 \%$ were complicated with pleurisy. $17,14 \%$ of cases required hospitalization longer than 20 days, with maintaining increase the level of procalcitonin $(10 \mathrm{ng} / \mathrm{mL})$.

\section{Conclusion}

The level of serum procalcitonin correlates with bacterial infections, severity of infections and in the same time is useful to predict the prognosis of our patients, both in complicated cases and those that have a favorable evolution after the treatment.

Keywords: procalcitonin, children, bacterial infection, lower respiratory tract infection, severity.

569

\section{Respiratory}

Conservative Management of Complicated Pneumonias in Children Angus Keeley ${ }^{1}$, Theofilos Polychronakis ${ }^{2}$, Robert Ross-Russell ${ }^{2}$ ${ }^{T}$ School of Clinical Medicine, University of Cambridge, UK ${ }^{2}$ Department of Paediatrics, Addenbrooke's Hospital, UK

\section{Background}

BTS guidelines for treatment of empyema recommend drainage, fibrinolysis and antibiotics, which has a similar efficacy to surgical intervention. Both strategies reduce the duration of illness but complications such as bronchopulmonary fistula are being increasingly described ${ }^{1}$

Objectives

To consider the role for treatment limited to antibiotics with outpatient assessment in those children who are stable, with complicated pneumonia demonstrated on CXR, even with large empyema or effusion.

Methods

We retrospectively reviewed all cases of paediatric empyema between December 2015 and August 2016 at a tertiary respiratory centre. We included only patients not treated in a high dependency unit.

\section{Results}

We identified 8 patients between $12 \mathrm{~m}$ and $10 \mathrm{y}$ old. All 8 had moderate to large pleural effusion with no substantial co-morbidity. Five had a long history of illness. Three had antibiotics in the community. Three had a septated effusion. Four patients were treated with IV antibiotics alone for 2-14 days. This was followed by oral antibiotic treatment of 2-4 weeks. Four patients underwent insertion of an $8 \mathrm{Fr}$ pigtail chest drain.1 patient was lost to follow up. In the remaining 3 patients treated with a chest drain, there was some degree of re-accumulation of the effusion after drain removal. No patients had surgical decortication. After re-accumulation, these patients had IV antibiotics for $2-4$ weeks. All 7 patients had complete resolution of effusion on imaging performed as an outpatient.

\section{Conclusion}

In moderate pleural effusion, even after failure of initial treatment or with loculation, conservative management with antibiotics alone can be considered as a viable alternative to progression to further invasive treatment. References

Sawicki GS et al. Necrotising pneumonia is an increasingly detected complication of pneumonia in children. Eur. Respir. J. 31, 1285-1291 (2008).

\section{7}

\section{Respiratory}

The Effects of Perinatal Vitamin D Levels on Lung Function Performance and Respiratory Infection in Infancy Shen-Hao Lai ${ }^{1}$, Sui-Ling Liao ${ }^{2}$, Ming-Han Tsai ${ }^{2}$, Man-Chin Hua ${ }^{2}$, ChihYung Chiu ${ }^{2}$, Kuo-Wei Yeh ${ }^{1}$, Tsung-Chieh Yao ${ }^{1}$, Jing-Long Huang ${ }^{1}$

${ }^{I}$ Department of Pediatrics, Chang Gung Memorial Hospital, Linkou Branch, Taiwan

${ }^{2}$ Department of Pediatrics, Chang Gung Memorial Hospital, Keelung Branch, Taiwan

\section{Background}

Perinatal vitamin D deficiency is associated with a higher risk of wheezing in childhood. However, the relationship between vitamin D levels and lung function in infancy has not been investigated. The aim of this study was to investigate the impact of perinatal vitamin D levels on respiratory function and outcome in infancy.

\section{Materials and Methods}

Full-term infants without any chronic diseases or major anomalies were enrolled in the Prediction of Allergies in Taiwanese Children cohort study. Maternal and cord blood was collected for determining the vitamin D level. Questionnaires were recorded at birth and 6 months. Infant lung function, including tidal breathing analysis, respiratory mechanics, and forced tidal expiration, was tested at 6 months of age.

Results

A total of 122 mother-infant pairs were enrolled in this study, and 71 infants underwent lung function testing at 6 months of age. Vitamin D levels in maternal and cord serum were highly correlated $\left(r^{2}=0.457, p\right.$ 0.0001). Infants with lower vitamin D levels in their cord serum had poorer lung function performance and a higher risk of a respiratory tract infection before the age of 6 months ( $p 0.01)$.

\section{Conclusion}

Although a high correlation was found between maternal and cord vitamin D levels, the effect on respiratory outcome was different. Our study is the first to show that low cord vitamin D levels significantly affect the respiratory function and the likelihood of a respiratory tract infection before 6 months of age.

711

Respiratory

"The effects of short course of systemic corticosteroids treatment in wheezy children"

Valdone Miseviciene $^{1}$, Gintare Liakaite ${ }^{1}$

${ }^{I}$ Department of Pediatrics, Lithuanian University of Health Sciences, Lithuania 


\section{Background}

The risk and benefits of long-term usage of systemic glucocorticosteroids (GCS) is widely analised. The short-term effects of systemic GCS still remain unclear.

\section{Aim and objective}

To analize the efects of short-term usage of systemic GCS on variation of blood cells count, biohemistry results and cortisol levels in children with acute wheezing.

\section{Methods}

Participants of prospective study were 44 wheezy children (average age-2.82 y/o; 3 months - $17 \mathrm{y} / \mathrm{o}$ ) treated at LUHSH Kauno Klinikos Pediatric Clinic. Intervention group - 26 children who received systemic GSC (methylprednisolone or prednisolone), control group - 18 children without GCS. Full blood count, biochemistry tests ( $\mathrm{Na}, \mathrm{K}$, glucose) and cortisol level in blood of all patients were analized. Tests were performed on the 1 st, 2 nd and 4th days of treatment. On the 1st day blood was taken before systemic GCS and any time of the day at the admittance. On other days - early in the morning. Results between 1st and 2nd day, and between 1st and 4th day were evaluated and compared. SPSS 23.0 statistics pack was used for data analysis.

\section{Results}

An average dose of methylprednisolone was $1.2(0.4-2) \mathrm{mg} / \mathrm{kg}$, duration of usage - $4.4(1-15)$ d. Short-term usage of systemic GCS increased sodium concentration $(p=0.014)$, decreased cortisol level $(r=0.252$; $p=0.081)$, the count of leukocytes $(p=0.043)$, neutrophils $(p=0.045)$, and eosinophils (p0.001) in the blood plasma. Systemic GCS caused the major reducion of eosinophils count in allergic children $(p=0.023)$. The higher age of the child was the risk factor for cortisol supression after four days of using systemic GCS ( $\mathrm{r}=-0.403 ; p=0.007)$.

\section{Conclusion}

The short-term usage of systemic GCS in wheezy children can reduce eosinophil's count in allergic patients as well as to decrease cortisol level especially in older children. Other minor effects on the level of sodium, leucocytes and neutrophils could be registred.

648

\section{Respiratory}

Neuroendocrine Cell Hyperplasia in Plastic Bronchitis: cause, effect or incidental finding?

Aine Lynch ${ }^{1}$, Andrew Nicholson ${ }^{4,5}$, Andrew Bush ${ }^{3,5}$, Paul McNally ${ }^{1,2}$

${ }^{T}$ Department of Respiratory Medicine, Our Lady's Children's Hospital, Crumlin, Ireland

${ }^{2}$ Department of Paediatrics, Royal College of Surgeons in Ireland, Ireland

${ }^{3}$ Department of Paediatric Respiratory Medicine, Royal Brompton and Harefield NHS Foundation Trust, UK

${ }^{4}$ Department of Histopathology, Royal Brompton and Harefield NHS Foundation Trust, UK

${ }^{5}$ National Heart \& Lung Institute, Imperial College, UK

\section{Introduction}

Plastic bronchitis $(\mathrm{PB})$ is a rare disorder characterised by the development of large branching casts of mucus, cells and fibrin, alone or combination. Casts typically cause large airway obstruction, sometimes requiring bronchoscopic removal. Reported mortality rates vary from $6 \%$ to $60 \%$, with higher rates in patients with underlying cardiac disease.

\section{Case Report}

We present the case of a previously well child presenting with a protracted history of recurrent acute presentations of airway obstruction from infancy. When well, bronchoscopy and chest imaging were normal. Episodes of airway obstruction, reportedly triggered by strong odours, were refractory to standard therapies and only resolved on expectoration of mucus plugs or casts. A diagnosis of idiopathic plastic bronchitis was made based on the clinical picture but there was no response to pulsed intravenous methylprednisolone, inhaled nebulised N-acetyl-cysteine, dornase alpha, tiotropium bromide, terbutaline or nedocromil. Cytology of casts shoed sheets of mature neutrophils and lung biopsy showed a mild eosinophilic bronchiolitis with an unexpected finding of neuroendocrine cell hyperplasia.

\section{Discussion}

Here we report on a girl with a unique airway disease, characterised by (1) chronic eosinophilic airway inflammation and increased airway neuroendocrine cells; and (2) recurrent acute neutrophilic airway inflammation with bronchial cast formation. Although there is evidence of airway eosinophilia, which might suggest allergic disease, the neutrophilic cytology of the casts in the acute attacks are against this hypothesis. Furthermore, no evidence of any significant allergic sensitization could be found. We speculate that this may be a late manifestation of neuroendocrine cell hyperplasia of infancy. It is interesting to speculate that these acute attacks may represent a disordered and immature airway response to noxious stimuli. The improvement in severity over time is supportive of this assertion.

309

\section{Respiratory}

$\mathrm{X}$-ray protection in pediatric radiology with special view on respiratory tract

Durdica Milkovic ${ }^{1}$, Maria Ranogajec-Komor ${ }^{2}$, Danica Batinic ${ }^{3}$

${ }^{T}$ Radiology, Children Hospital Srebrnjak, Croatia

${ }^{2}$ Dosimetry Laboratory, Institute Rudjer Boskovic, Croatia

${ }^{3}$ Pediatrics, Children Hospital Srebrnjak, Croatia

\section{Background}

There is permanently growing demand in diagnostic radiological procedures in pediatrics. Important issue is to know precisely effects of dose radiation in children.

Objective was to investigate effect of low dose radiation during X-ray examination of chest in children.

Methods X-ray of chest was eximinated in patients and phantoms. The influence of low doses of radiation on chest and other organs, as well as effect of working method and human factor were analysed. Measurements were done with termoluminiscent dosimeters (TL) and radiophotoluminiscent dosimeters (RPL) adopted to age of children. Babies and small children were exposed in baby-fix and rotation chair, and older children stand in front of camera. The doses were measured in and on phantom (size of 3 years old child) too. The doses were compared with those of patients. Also, were investigated potential DNA damage.

\section{Results}

Mean value X-ray dose in primary beam on chest $\mathrm{x}$-ray in patients was $0.26 \mathrm{mSv} \pm 0.11 \mathrm{mSv}$ and in phantom $0.66 \pm 0.17 \mathrm{mSv}$. Correlation was found between doses in primary beam and DNA damage. We found corelation between working method of technicans and X-ray doses too.

\section{Conclusion}

Our long term work shows importance of permanent dosimetry in X-ray diagnostics in children. Continuous eduaction and good teamwork for dose reduction to minimize the potential damage is necessary to maintain the optimal benefit for patients especially children and medical staff too.

795

\section{Respiratory}

Antibiotic use in tonsillitis

Nedelcuta Ramona ${ }^{1}$, Calin Gigi ${ }^{1}$, Popescu Mirela ${ }^{1}$

${ }^{T}$ Pediatrics, UMF Craiova, Romania

\section{Background}

A group B hemolytic streptococcus is no longer a rarity in pediatric clinics. Often, due to somewhat laborious culture techniques, in the absence of clinical suspicion of etiology, a group A hemolytic streptococcus 
can pass undiagnosed or mistakenly labeled as a hemolytic staphylococcus aureus; especially in cases of coexistence of double etiology.

Sometimes the diagnosis is retrospective, as in the case of post-scarlet desquamation or absolutely random, when we detect a murmur in endocarditic rheumatic fever.

\section{Objective}

As a personal observation, in the cases studied, unlike the classical Pediatrics, both the age of diagnosis (often under 1 year) and the antibiotic response in the antibiotic were displaced, meaning that most of the strains are resistant to penicillin, erythromycin, even clarithromycin.

Methods

1-year retrospective study in a total of 162 patients with haemolytic streptococcal disease, the majority of them (131) being of group A. The primary age of screening is that of the school and pre-school child, about $20 \%$ of whom are infants.

\section{Results}

No significant differences in sex distribution were observed. Most cases were diagnosed in the cold months, over $80 \%$ between may and september, with an average of $4-5$ cases/month

Over $75 \%$ of antibiotics showed "paradoxical" sensitivity to unobserved "classical" antibiotics for the pathogen (Augmentin, Cefuroxim, Gentamicin) and penicillin-resistant: 2 non-group A streptococci were streptococcus pneumoniae.

\section{Conclusion}

Repositioning attitudes towards antibiogram therapy in all children with a streptococcal infection due to "surprises" with regard to antibiotic susceptibility. Reassessing the ASLO titre for retrospective diagnosis and targeted treatment. Promotion of effective hygiene rules in the community.

Elimination of taboos relating to age of diagnosis and response to therapy. Applicability of fast diagnostic kits.

737

\section{Respiratory}

A Brief Smoking Intervention on Caregivers to Reduce Tobacco Smoking Exposure in Asthmatic Children: Evaluating Its Effectiveness and Exploring Barriers to Successful Smoking Cessation Alicia Ong ${ }^{1}$, Gaun Tain Lee ${ }^{2}$, Mary Hoon ${ }^{2}$, Derrick Lian ${ }^{2}$, Thomas Biju ${ }^{2}$, Oon Hoe Teoh ${ }^{2}$, Oh Moh Chay ${ }^{1,2}$

${ }^{1}$ Medicine, Duke-NUS Medical School, Singapore

${ }^{2}$ Department of Respiratory Medicine Service, KK Women's AND

Children's Hospital, Singapore

\section{Introduction}

Parental smoking exposure is proven to be a significant modifiable factor in pediatric asthma exacerbations. Institutions worldwide have worked on reducing tobacco smoke exposure by counselling caregiver smokers. However, the existing brief smoking cessation program at KK Women's and Children's Hospital, has yielded a low take-up rate for intensive smoking cessation program and 6-month quit rate.

\section{Objective}

This study aims to evaluate the effectiveness of a modified brief smoking cessation program and explore barriers to successful smoking cessation. Methods

Our study adopts a mixed method approach. Caregiver smokers were invited to go through the modified brief counselling followed by an optional intensive smoking cessation. Survey forms were given, at four specific time points - before and after the brief counselling, at 3 months and at 6 months. Variables such as quit rates, knowledge on smoking, motivation to quit, nicotine dependence and asthma control of child, were assessed using relevant validated questionnaires.

Secondly, one-on-one in-depth interviews were conducted with healthcare providers and caregiver smokers, to evaluate their attitudes and perspectives on parental, until saturation is reached. Thematic coding was utilized to present the themes elicited.

\section{Results}

Eighty-one met the inclusion criteria and were enrolled in the quantitative component of study. Twenty-one declined and 53 were recruited in the study. One subject withdrew and 52 are currently under follow-up.

Preliminary analysis shows that there is statistical significant difference in take-up rate for intensive smoking cessation program at $15.1 \%$, as compared to previous method, at $4.58 \%$ (p0.05).

For the qualitative component, 16 participants were recruited in the study. Thematic coding is underway.

\section{Conclusion}

Preliminary data from this pilot prospective study suggests that the modified brief smoking cessation counselling may increase the smokers' motivation to quit. However, longer follow-up is required to determine if there is an increase in quit rate and its impact on pediatric asthma. (Follow-up data up to 3-month and completed qualitative analysis will be ready by October 2017.)

\section{9 \\ Respiratory}

Bronchial asthma and obesities in children - the connection between the "social epidemics"

Guergana Petrova ${ }^{I}$, Penka Perenovska ${ }^{I}$, Dimitrinka Miteva ${ }^{I}$, Snezhina Lazova', Vera Papochieval

${ }^{1}$ Pediatric clinic, UMHAT "Alexandrovska; Medical University, Sofia, Bulgaria

\section{Background}

Bronchial asthma (BA) and obesities show dramatically risen incidence over the years. In USA for a period of 16 years (1980-1996) BA incidence rose from $7.4 \%$ to $9.3 \%$. In the meantime for 30 years the number of obese children had tripled (from $7 \%$ to $18 \%$ ) and the one of obese adolescents had quadrupled (from $5 \%$ to $21 \%$ ). There is still a debate whether there is a connection between both diseases. Inhaled corticosteroids (ICS) are the recommended therapy for BA in children. Obesity is considered a risk factor for asthma in the same way as stigmatizing ICS for increased weight. Methods

We evaluated the medical data and weight of 200 children (aged 5 to 17 years) admitted for asthma attack regarding the asthma severity, the natural history of asthma and inhaled corticosteroid (ICS) treatment period. As a control group we used age and sex matched children without history of asthma or other allergic diseases.

\section{Results}

There were not any significant correlations between weight and the ICS treatment, family history or atopy status (p0.05). When we stratified the asthma children according the ICS use, surprisingly those who are not on ICS therapy have a tendency to be heavier than children on ICS or controls ( $p=0.06 \mathrm{BA}$ on ICS vs BA without ICS; $p=0.07 \mathrm{BA}$ without ICS vs controls). Stratifying according the asthma control - those with good control don't show difference from the control group, while cases with poor control are with tendency to be overweight vs controls $(p=0.054)$. Stratifying according the weight we found worse control in both overweight and underweight children compared to those with normal weight. Conclusion

We assume that long-term ICS therapy is not that determining for weight status in BA patients. Children, with good control, optimal dose ICS with proper inhalation technique, following regular exercise regimen and proper diet can maintain optimal weight, which in turn supports better control.

423

\section{Respiratory}

Quality of life in children with chronic disease - should we take it into consideration?

Guergana Petrova ${ }^{I}$, Penka Perenovska ${ }^{l}$, Dimitrinka Miteva ${ }^{l}$, Vera Papochieva', Snezhina Lazova ${ }^{l}$, Silvia Shopova ${ }^{1}$

${ }^{1}$ Pediatric clinic, UMHAT "Alexandrovska; Medical University, Sofia, Bulgaria 


\section{Background}

Chronic disease regardless of its nature, leaves a mark on quality of life (QoL). QoL is still relatively poorly studied and underestimated problem in Bulgaria for children with chronic respiratory diseases.

\section{Methods}

We analyzed 77 families of children with chronic illnesses: bronchial asthma (BA) $(n=41)$ and cystic fibrosis $(\mathrm{CF})(n=36)$. A short version of the WHO questionnaire for QoL was used. All 31 questions were analyzed separately and as whole, as well we compared them two by two. Due to heterogeneity of the matters in the questions we grouped them in several areas to analyzed different aspects of life: physical appearance, emotional health, social interactions, health care and social status.

Results

CF patients evaluated their QoL average $62.75 \% \pm 23.75 \%$, while those with BA - $81.4 \% \pm 16 \%(p=0.045)$. There was a significant correlation between QoL and sense of joy (spearman rho $=0.529, \mathrm{p} 0.05$ ). We confirmed a significant correlation between awareness and financial security. The need for treatment is directly related to physical pain. Marked correlations between coping with negative feelings and overcome problems; the social relationships and support from relatives; between ability to work and sound sleep; daily activities and recreation were noted.

\section{Conclusion}

The decrease in the results of QoL is predictive sign of more frequent need of in-hospital treatment. The determination of QoL should not be neglected. It provides unique information about the impact of the disease and the effects of various therapies for patients with chronic diseases, whose treatment aims to improve daily functioning and well-being of the patient as a whole.

\section{8}

\section{Respiratory}

Vitamin D status in young children with viral croup Maria Romanko ${ }^{1}$, Marina Mamenko ${ }^{1}$, Vyacheslav Berezhny ${ }^{1}$

${ }^{T}$ Department of Pediatrics № 2, Shupyk National Medical Academy of Postgraduate Education, Ukraine

\section{Background}

Vitamin D is a regulator of calcium and phosphate homeostasis and has numerous extra-skeletal effects. Therefore, vitamin D represents a potentially useful intervention for combating viral infection.

The aim

To evaluate the impact of vitamin D status of young children on the viral croup occurrence.

\section{Materials and methods}

Hospital-based survey included 80 children aged 1-36 months with viral croup. The evaluation of vitamin D status was conducted by measuring serum 25(OH) D levels.

\section{Results}

Median of serum $25(\mathrm{OH})$ D levels in young children with viral croup was $31.8 \mathrm{ng} / \mathrm{ml}\left(\mathrm{Q}_{1}=18.4 ; \mathrm{Q}_{2}=47.4\right)$. The rate of recurrent infections was $43.8 \pm 5.5 \%(35 / 80)$. The levels of $25(\mathrm{OH}) \mathrm{D}$ in patients with frequent respiratory viral infections was significantly lower $\left(25.4 \mathrm{ng} / \mathrm{ml} ; \mathrm{Q}_{1}=18.1\right.$; $\left.\mathrm{Q}_{3}=40.1\right)$ than lose in children with first episode of viral infection $\left(38.3 \mathrm{ng} / \mathrm{ml} ; \mathrm{Q}_{1}=19.6 ; \mathrm{Q}_{3}=47.7\right), p=0.09$. Patients with moderate viral croup had significantly lower levels of $25(\mathrm{OH}) \mathrm{D}\left(25.5 \mathrm{ng} / \mathrm{ml} ; \mathrm{Q}_{1}=15.2\right.$; $\left.\mathrm{Q}_{3}=38.6\right)$ than children with mild clinical performance of disease $\left(43.2 \mathrm{ng} / \mathrm{ml} ; \mathrm{Q}_{1}=30.8 ; \mathrm{Q}_{3}=61.5\right), p=0.0001$. The levels of $25(\mathrm{OH})$ $\mathrm{D} 40 \mathrm{ng} / \mathrm{ml}$ were associated with having higher odds of severe performance of viral croup $(\mathrm{OR}=7.56 ; 95 \% \mathrm{CI}: 2.68-21.3) .78 .8 \pm 4.6 \%(63 / 80)$ of patients had the first episode of viral croup whereas recurrent episodes of illness were detected in $21.3 \pm 4.6 \%$ (17/80) of cases. Children with levels of serum $25(\mathrm{OH}) \mathrm{D} 40 \mathrm{ng} / \mathrm{ml}$ had higher odds of recurrent episodes of viral croup $(\mathrm{OR}=12 ; 95 \%$ CI: 1.5-96.15), $p=0.011$.

\section{Conclusion}

Low levels of vitamin D in young children are associated with higher odds of severe performance and recurrent episodes of viral croup.

\section{5}

Respiratory

\section{An Unusual Complication of Mycoplasma Infection}

Louise Selby $^{1}$, Catriona Middleton ${ }^{1}$, Theofilos Polychronakis ${ }^{2}$

${ }^{T}$ Paediatric Respiratory, Cambridge University Hospitals NHS

Foundation Trust, UK

${ }^{2}$ Paediatric Respiratory, Royal Brompton and Harefield Hospitals NHS Foundation Trust, UK

We present the case of an 8 year old boy with an unusual complication of mycoplasma pneumonia. He presented to his local hospital with cough, coryza and fever for 9 days. He developed significant respiratory distress and commenced intravenous antibiotics, but required escalation of respiratory support to optiflow, CPAP, then required intubation and ventilation and further escalation to high frequency oscillation.

Initial chest $\mathrm{x}$-ray showed dense consolidation of the right lung, which progressed to a right sided white out.

He was treated with intravenous antibiotics with atypical cover. Pleural tap was negative for bacterial growth and nasopharyngeal aspirate for extended respiratory viruses was negative, but serology was positive for mycoplasma pnuemoniae.

He developed a swollen right leg; Doppler ultrasound showed thrombus from the right external iliac to the popliteal vein. He commenced anticoagulation and his case was discussed with a quarternary centre. He had a CTPA performed showing extensive bilateral pulmonary emboli, consolidation and infarction with hepatomegaly.

After 3 weeks he was extubated to CPAP and remained tachypnoeic but not hypoxic. An IVC filter and clot extraction were discussed because of sub-therapeutic Anti Xa levels, however it was felt the risks outweighed the benefits. He remained on bed rest until his factor Xa level was therapeutic due to risk of further emboli. Repeat CTPA was performed in view of persistent temperatures and to reassess the clot burden and right lung. This showed consolidation and cavitating infarctions with an enlarging effusion.

Conservative management with intravenous antibiotics was continued; two weeks later oral antibiotics and warfarin were introduced. His pulmonary pressures normalised on echocardiogram and he continued to make good progress.

There are cases of pulmonary infarction secondary to mycoplasma infection in the literature. We present a complicated course and interesting images requiring multi-disciplinary and multi-site discussions eventually achieving excellent outcomes.

\section{6}

\section{Respiratory}

A One-Year Prognosis of Preschool Children Hospitalized with Acute Wheezing in Thailand

Paskorn Sritipsukho ${ }^{1}$, Khlongtip Matchimmadamrong ${ }^{2}$, Sasawan Chinratanapisit ${ }^{3}$, Jitlad da Deerojanawong ${ }^{4}$

${ }^{I}$ Pediatrics, Faculty of Medicine, Thammasat University, Thailand

${ }^{2}$ Pediatrics, Saraburi Hospital, Thailand

${ }^{3}$ Pediatrics, Bhumibol Adulyadej Hospital, Thailand

${ }^{4}$ Pediatrics, Faculty of Medicine, Chulalongkorn University, Thailand

\section{Background}

Wheezing in preschool children is a common symptom. Genetic with environmental factors might be responsible for their prognosis.

\section{Objective}

The study aimed to determine an incidence of recurrent wheezing among preschool children who hospitalized with acute wheezing after a follow- 
up period of 12 months. Factors associated with recurrent wheezing were explored.

\section{Method}

A longitudinal study was conducted among 240 children, aged between 6 months and 5 years, who hospitalized with acute wheezing in 4 hospitals located in Bangkok and adjacent provinces, Thailand. Demographic, house environments and clinical characteristic data were collected at entry. Serum specific IgE levels against common food and inhalant allergens and serum 25-hydroxyvitamin D concentrations were measured. All children were followed every 4 months for 12 months to record emergency or unscheduled visits due to recurrent wheezing. Exploratory analysis by multiple logistic regressions was used to determine significant factors associated with recurrent wheezing.

\section{Results}

At entry, the mean age was 24.4 months ( $\mathrm{SD}=15.7$ months) with 148 boys $(62.7 \%)$. Ninety-four cases $(40.9 \%)$ were hospitalized with the first wheezing episode of their life. By laboratory results, 197 (82.8\%) and 56 $(24.9 \%)$ children were atopic and had vitamin D insufficiency respectively. The one-year incidence risk of emergency visits due to recurrent wheezing among those with the first wheezing episode and those with history of previous wheezing at entry were $42.3 \%$ (95\% confidence interval: $31.2 \%-54.0 \%$ ) and $55.6 \%$ (95\% confidence interval: $46.1 \%-64.9 \%$ ) respectively. By multivariate analysis, being the second child or more, vitamin D insufficiency, previous wheezing, and allergic rhinitis were significantly associated with recurrent wheezing with the adjusted odds ratios of 2.5 (95\% confidence interval: 1.3-5.3), 2.3 (95\% confidence interval: 1.1-4.5), 1.9 (95\% confidence interval: $1.1-$ 3.5 ), and 1.6 (95\% confidence interval: 1.3-2.9) respectively.

\section{Conclusion}

Recurrent wheezing in preschool children was common. Being the second child or more in the family, vitamin D insufficiency, previous wheezing, and allergic rhinitis were significant risks of recurrent wheezing.

\section{8}

\section{Respiratory}

Risk Factors of Recurrent Pneumonia in Under-five Children Hospitalized at Hasan Sadikin Hospital

Filla Suryaningrat ${ }^{I}$, Heda Melinda Nataprawira ${ }^{I}$

${ }^{T}$ Faculty of Medicine/dr. Hasan Sadikin General Hospital, Departement of Child Health, Indonesia

\section{Background}

Pneumonia is the highest cause of deaths among underfive children particularly in developing countries. Among children with pneumonia, many suffer from recurrent pneumonia (RP). Several studies reported that increase risk of recurrent pneumonia is associated with underlying diseases. Surprisingly, to date there are few primary studies investigating the risk factors of recurrent pneumonia particularly in developing countries.

Objective

To determine the risk factors of recurrent pneumonia in under 5 years

Methods

We assessed possible risk factors of recurrent pneumonia in a case control study of underfive patients admitted to Hasan Sadikin General Hospital, Indonesia from January 2014 to December 2016. Databases of patients underfive who were admitted 2 times or more for pneumonia were documented Control subjects were children (age and year of admission matched) with pneumonia but not recurrent. Bivariate statistical analysis was conducted to analyze prominent risk factors.

\section{Results}

Pneumonia in underfive children was $740(95.73 \%)$ of 773 pneumonia patients in children. Recurrent pneumonia was documented in $88 / 740$ $(11.89 \%)$ underfive children with pneumonia. Among the risk factors associated with recurrent pneumonia, several were found to be statistically significant (P0.001) including underlying congenital heart disease
$(\mathrm{OR}=3.46 ; 95 \%$ CI: $1.57-7.71)$, cerebral palsy $(\mathrm{OR}=5.56,95 \%$ CI: $1.87-17.69)$, HIV state (OR $=41.93 ; 95 \%$ CI: 5.85-848.83), malnutrition $(\mathrm{OR}=3.79,95 \% \mathrm{CI}: 1.86-7.80)$, and residing in an overcrowded area $(\mathrm{OR}=4.75,95 \% \mathrm{CI}: 2.32-9.78)$. Other risk factors that were analyzed but was not found statistically significant included prematurity, weight at birth, history of immunization, exposure to smoke, house pet, and other conditions such as hypothyroid and Down syndrome.

\section{Conclusion}

As a preventable disease, recurrent pneumonia may be controlled by addressing personal and environmental risk factors. Measures such as improving child's nutritional state; educating and providing additional support for parents of children with congenital heart disease, cerebral palsy, and HIV may be done to prevent recurrent pneumonia.

Keywords. Recurrent, pneumonia, children, indonesia

\section{2}

\section{Rheumatology}

Osteoarhritis of Temporomandibular Joint in Children and Adolescents Compared with Adult Patient Population

Tomislav Badel ${ }^{1}$, Ivana Savić Pavičin ${ }^{2}$, Nenad Lukić ${ }^{3}$, Jelena Valpotić, $\overline{\text { Dijana Zadravec }}$

${ }^{1}$ Department of Removable Prosthodontics, School of Dental Medicine, University of Zagreb, Croatia

${ }^{2}$ Department of Dental Anthropology, School of Dental Medicine, University of Zagreb, Croatia

${ }^{3}$ Clinic of Masticatory Disorders, Removable Prosthodontics and Special Care Dentistry, Center of Dental Medicin, University of Zurich, Switzerland

${ }^{4}$ Department of Paediatric and Preventive Dentistry, Outpatient Dental Clinic "Zagreb", Croatia

${ }^{5}$ Department of Diagnostic and Interventional Radiology, Clinical Hospital Center "Sestre milosrdnice", University of Zagreb, Croatia

\section{Background}

Osteoarthritis (OA) is a low-inflammatory arthritic condition that results in various degenerative joint changes clinically manifested as crepitation and arthralgia of the temporomandibular joint (TMJ) with limited opening of the mouth.

\section{Objective}

The aim of this study was to compare clinical characteristics of children and adolescents in the sample of general patient population with OA of TMJ.

\section{Methods}

A prospective study was carried out between January 2001 and December 2015 and it included 174 patients with OA of TMJ. The sample of patients was divided in two groups: 6 patients aged under 20 (mean age of 17.7) and the remaining 168 patients (mean age of 50.2) who were seeking treatment. Pain intensity (at first examination T0) in TMJ was shown on the visual-analogue scale (VAS; 0 , no pain; 10 , the most severe pain). They were treated by an occlusal splint and/or physical therapy with a sixmonth (T1) and one-year (T2) follow-up. Definitive TMJ-diagnoses were confirmed by magnetic resonance imaging (MRI).

\section{Results}

There was no statistical difference between average pain at $\mathrm{T} 0 \mathrm{in}$ patients over 20 (6.2) and patients aged up to 19 (6.4) evaluated by VAS (p0.05). In the beginning, the pain by VAS amounted to T1 in patients aged up to 19 on 1.5 and in patients over 20 on $2.5(\mathrm{p} 0.05)$ and at T2 patients aged up to 19 had no pain (0) and for patients aged over 20, the pain was 1.7 (p0.001). MRI findings for patients aged up to 19 were: predominately erosions of the condylar head and anterior disc displacement without reduction in all patients.

\section{Conclusions}

OA was rarely observed in very young TMJ patients and MR images showed no osteophyte formation on TMJs. However, the prognosis of TMJ treatment is better for young patients compared to that for the older ones. 
646

\section{Rheumatology}

Particular issues of liver function in children with Systemic Lupus Erythematosus

Tatiana Golovko ${ }^{I}$, Tatiana Zimnitska ${ }^{1}$, Iryna Zuravlova ${ }^{l}$

${ }^{T}$ Department of Pediatrics, V.N.Karazin Kharkiv national university, Ukraine

\section{Introduction}

Systemic lupus erythematosus (SLE) is referred to be as an autoimmune inflammatory connective tissue disorder. Liver has important role in proteins metabolism and inflammatory process.

Objective

Assessment of liver function in children with SLE with due regard to disease duration was goal of the research.

\section{Materials and methods}

The study involved 12 children with SLE aged from 7 to 18 years. Patients were divided into 2 groups according to disease duration. First group cases with anamnesis up to 5 years were added and 2 nd group contained patients with disease duration from 5 to 11 years. Liver function was investigated by measurement of alanine aminotransferase (ALAT), aspartate aminotransferase (ASAT), haptoglobin, triglycerides, gamma glutamine transferase (GGT), apolipoprotein-A (Apo-A), a2-macroglobulin, cholesterine levels. The equation for calculating the FibroTest score regression coefficient had been done according U.S. patent 6,631,330.

\section{Results}

Thirty-three percent of patients had enlargement of liver in both comparative groups according physical examination and ultrasound (US). The level of a2-macroglobulin, Apo-A, total bilirubin did not depend on duration of disease and was within normal limits. Eight percent of children with SLE had increased GGT content. Frequency of increased rate of haptoglobin was the same in the both groups $(17 \%)$ and also was associated with prolonged inflammatory process. Half of patients in both groups had increased level of cholesterine and $17 \%$ of children were presented with increased triglycerides rate. It can be assessed as a risk of yearly development of atherosclerosis. In spite of involvement of liver, which was proved by physical examination, US and biochemical tests, nobody from investigated patients had fibrosis (corresponding to Fibro Test).

\section{Conclusion}

Children with SLE have risk of atherosclerosis development. Liver function damage does not depend on SLE duration. Liver fibrosis was not diagnosed in patients with long duration of disease.

\section{6}

\section{Rheumatology}

Amplified Pain Syndromes In Children: Characteristics of a singlecenter cohort

Jitka Obrsalova ${ }^{I}$, Marketa Vranova $^{I}$, Sarka Fingerhutova ${ }^{I}$, Jiri Zeman ${ }^{1}$, Pavla Dolezalova

${ }^{1}$ Department of Paediatrics and Adolescent Medicine, General University Hospital and 1st Medical Faculty, Charles University, Paediatric Rheumatology Unit, Czech Republic

\section{Background}

Among defined amplified pain syndromes chronic regional pain syndrome and fibromyalgia have been recognised. In children they are often less clear-cut than in adults and the terms localized and diffuse idiopathic pain syndrome (LIPS,DIPS) have been adopted. They are characterized by intermittent or persistent musculoskeletal pain without identifiable aetiology accompanied with significant degree of physical and psychosocial dysfunction.

\section{Objective}

To analyze clinical features of a cohort of patients diagnosed with amplified pain syndromes over the 2 year period

\section{Methods}

Consecutive patients up to 18 years of age diagnosed between March/2015 and February/2017 were recruited. Upon exclusion of organic aetiology LIPS was diagnosed in the presence of 1-2 painful regions with hyperaesthesia and/or autonomic changes while DIPS patients had 3 or more painful areas or widespread pain for longer than 3 months. Following domains were assessed: Pain(VAS 0-100 mm), fibromyalgia( $\geq 11 / 18$ tender points), skin sensitivity and autonomic changes, physical function(CHAQ, activities of daily living for upper and lower extremities), Beighton scale for hypermobility $(\geq 6 / 9)$, muscle strength (CMAS 0-52), psychosocial wellbeing(PedsQL).

\section{Results}

Patient characteristics are shown in the Table. Significant degree of pain was reported by all patients. Lower extremities were commonly affected (80\% LIPS and $94 \%$ of DIPS) with the knee area predominating. Autonomic changes were more frequent in LIPS while hypermobility and fibromyalgia tender points were more prevalent in DIPS patients. Significant disability (e.g. crutches, wheel-chair) was present in $23(72 \%)$ patients as reflected by CHAQ scores.

\section{Conclusion}

Children and adolescents with amplified pain represent a difficultto-manage patient subgroup. High level of suspicion and physicians' awareness of these disabling conditions are crucial for their early recognition and complex treatment that combines vigorous physiotherapy with individually-tailored psychological therapies. Prospective follow-up of these patients is a subject of ongoing study.

\begin{tabular}{|c|c|c|c|}
\hline & LIPS & DIPS & total \\
\hline$N(96)$ & $15(47)$ & $17(53)$ & 32 \\
\hline Girls N (96) & $12(46)$ & $14(54)$ & $26(81)$ \\
\hline Boys N (\%) & $3(50)$ & $3(50)$ & $6(19)$ \\
\hline Mean age at onset - years (SD) & $13(3.1)$ & $14,5(2.2)$ & $12,7(3.8)$ \\
\hline Mean time to diagnosis - months (SD) & $5,6(7.2)$ & $14(19.9)$ & $10(15.9)$ \\
\hline \multicolumn{4}{|l|}{ Patient-reported measures mean(SD): } \\
\hline - Pain VAS (0-100) & $60,7(26.3)$ & $63,6(22)$ & $62,3(24.14)$ \\
\hline - $\operatorname{CHAQ}(0-3)$ & $1,42(0.73)$ & $1,38(0,88)$ & $1,39(0.82)$ \\
\hline - PedsOL (0-92) & $37,2(12.6)$ & $39,5(14,5)$ & $38,44(13.7)$ \\
\hline \multicolumn{4}{|l|}{ Examination: } \\
\hline - Fibromyalgia+ N(\%) & $3(20)$ & $12(80)$ & $15(47)$ \\
\hline - Hypermobility $+\mathrm{N}(\$ 6)$ & $3(33)$ & $6(67)$ & $9(28)$ \\
\hline - Skin sensitivity score $(0-6)$ mean (SD) & $2(0.97)$ & $2,3(0,87)$ & $2,15(0,94)$ \\
\hline - Autonomic change score (0-5) mean (SD) & $0,73(0,9)$ & $0,41(0.77)$ & $0,56(0,86)$ \\
\hline - Muscle strength CMAS $(0-52)$ mean (SD) & $48,5(3.6)$ & $43(9.5)$ & $45,7(7.9)$ \\
\hline - ADL upper (1-6) mean (SD) & $1,8(1.7)$ & $1,5(0.9)$ & $1,65(1.36)$ \\
\hline - $\quad A D L$ lower (1-8) mean (SD) & $4,5(1.8)$ & $3,8(1.85)$ & $4,16(1.87)$ \\
\hline
\end{tabular}

\section{Supported by CPS JEP}

598

Rheumatology

Pattern of bony involvement in an Irish cohort with chronic recurrent multifocal osteomyelitis

Daire O'Leary ${ }^{l}$, Anthony Gerard Wilson ${ }^{2}$, Emma-Jane MacDermott ${ }^{3}$, Orla Killeen $^{3}$

${ }^{I}$ Department of Paediatrics, RCSI, Ireland

${ }^{2}$ School of Medicine, UCD, Ireland

${ }^{3}$ National Centre for Paediatric Rheumatology, OLCHC, Ireland

\section{Background}

Chronic recurrent multifocal osteomyelitis (CRMO) is an autoinflammatory condition primarily affecting children with an estimated prevalence of 1 per $10^{6}$ (OMIM 259680). It is characterized by relapsing episodes of localized bone inflammation. Since 2006, 43 patients have been diagnosed with CRMO at the National Centre for Paediatric Rheumatology (NCPR).

Objectives

To describe the pattern of bone involvement in this cohort. 


\section{Methods}

Retrospective chart review.

\section{Results}

The median age at diagnosis was 10.5 years (range 6.8-15.4 years) with a male-to-female ratio of 1:2.6. All patients underwent MRI imaging of the symptomatic region and $86 \%$ had whole body MRI performed. Bone biopsy was performed in $79 \%$ of patients with $17 \%$ requiring a repeat. Biopsy was indicated when one or more of the following circumstances occurred; diagnostic uncertainty following regional or whole body MRI, patient unable to receive contrast agent for MRI, or whole body MRI unavailable. The mean follow-up period was 5.05 years.

The distribution of 264 documented lesions was as follows: lower limb bones $46.2 \%$ (23.1\% tibia, $10.6 \%$ femur), $23.1 \%$ axial skeleton primarily the vertebrae $(17.8 \%$ ), pelvic girdle $16 \%$, shoulder girdle $6.8 \%$ (predominantly clavicular lesions), and upper limb lesions accounted for only $8 \%$. Most patients $(86 \%)$ had multifocal disease.

\section{Conclusion}

Long bones of the lower limb were most frequently affected which is in keeping with previous reports. The relatively high incidence of vertebral lesions is significant as these carry a risk of morbidity from vertebral collapse or fracture.

\section{5}

\section{Rheumatology}

Comparative Characteristics of the of X-RAY, Ultrasound and MRI Examination Results in children with Arthritis Yuliia Bondarets ${ }^{I}$, Ivanna Romankevych ${ }^{1}$, Viacheslav Berezhnyi ${ }^{1}$ ${ }^{T}$ Pediatrics Dept.\# 2, Shupyk National Medical Academy of Postgraduate Education, Ukraine

\section{Background}

Joints diseases in children aren't rare and demand complex diagnostic process. The most routine methods of pathological changes detection in joints are X-ray, ultrasound (US) and magnetic resonance imaging (MRI). Sometimes there is necessity in using more than one of them, because each reveals only some spectrum pathological changes.

Because of a small number of data and short experience of this methods application in children, investigation of X-ray, US and MRI results is really actual.

\section{Objection}

To compare X-Ray, US and MRI data in children with arthritis.

\section{Materials and methods}

Twenty-two children, 15(68\%) with juvenile rheumatoid arthritis, $6(27.3 \%)$ with reactive arthritis and $1(4.5 \%)$ child with Legg-CalvePertes's disease. Mean age was $8 \pm 1.2$ years and mean duration of disease $1.36 \pm 0.3$ years.

\section{Result}

Bone structure changes were found in $2(9 \%)$ cases of osteoporosis and $1(4.5 \%)$ of femoral head consolidation and were detected only by X-ray. Joint space changes registered in 7(31.5\%) children by X-ray, 22(100\%) by US and MRI $(p=0.05)$. Joint effusion was in $5(22.7 \%)$ on X-ray and in $22(100 \%)$ on US and MRI $(p=0.05)$ cases. Pathological changes of synovial membrane found in 10(45.5\%) and increased blood flow in 18(82 \%) children during only on US scanning. Narrowing of cartilage height we saw in 1child (4.5\%) on MRI. Enthesitis was diagnosed by US in 1(4.5\%) and soft tissues consolidation were detect in 8(36\%) during X-ray. We didn't find any incidents of erosions and pannus in our investigation.

Discussion

Application of visualization methods for differentiation diagnostics in children with arthritis are important and informative. In addition, US is more available to use than MRI.

\section{Conclusions}

On early stages of diagnostic process in children with arthritis we recommend using X-ray and US examination. MRI has advantage in imaging of soft tissues, especially cartilages and tendons and in complicated cases.
162

Rheumatology

From fibrosis to diagnosis

Justyna Roszkiewicz ${ }^{I}$, Elzbieta Smolewska ${ }^{l}$

${ }^{T}$ Department of Pediatric Rheumatology, Medical University of Lodz, Poland

\section{Background}

Microscopic polyangitis is a rare autoimmune disease characterized by necrotizing inflammation of small-size vessel walls.

\section{Case report}

We herein present a case of 6-year-old girl whose way to establishing a proper diagnosis may be summed up by the phrase "from fibrosis to diagnosis". The history of our patient starts in the Department of Pulmonology where she was refered by her GP due to persistent crepitations at the base of both lungs, resistant to the antibiotic treatment. A CT scan performed there revealed that those auscultation changes were not the result of, as primarily thought, infective process but manifestations of massive lung fibrosis. What is more, at that time another symptom appeared - haematuria. In the search of a cause of lung fibrosis with an early onset in the differential diagnosis many pulmonary diseases were excluded (cystic fibrosis, Goodpasture syndrome, $\alpha-1$ antitrypsin deficiency, congenital abnormalities of surfactant homeostasis). Due to the presence of antinuclear antibodies in the patients serum the girl was transferred to Department of Rheumatology with suspicion of systemic inflammatory connective tissue disease. In the laboratory tests performed there presence of antinuclear antibodies was not confirmed, but what draw attention was existence of $\mathrm{p}$-ANCA antibodies in high titer. What is more, haematuria with the tendency to increase was also noted. Due to the complicated overall clinical picture and ambiguous laboratory tests results the biopsy of the lung and kidney tissue was performed, which shoved features of microscopic polyangitis in the former and glomerulonephritis in the latter. Finally, the diagnosis of microscopic polyangitis was established and glucocorticosteroids and cyclophosphamide pulses were introduced into the treatment with good clinical response.

\section{Conclusion}

Due to the fact that microscopic polyangitis is a very rare disease in children and can affect almost every organ of the human body the diagnostic and therapeutic process of this disorder is challenging and usually requires a multidisciplinary approach.
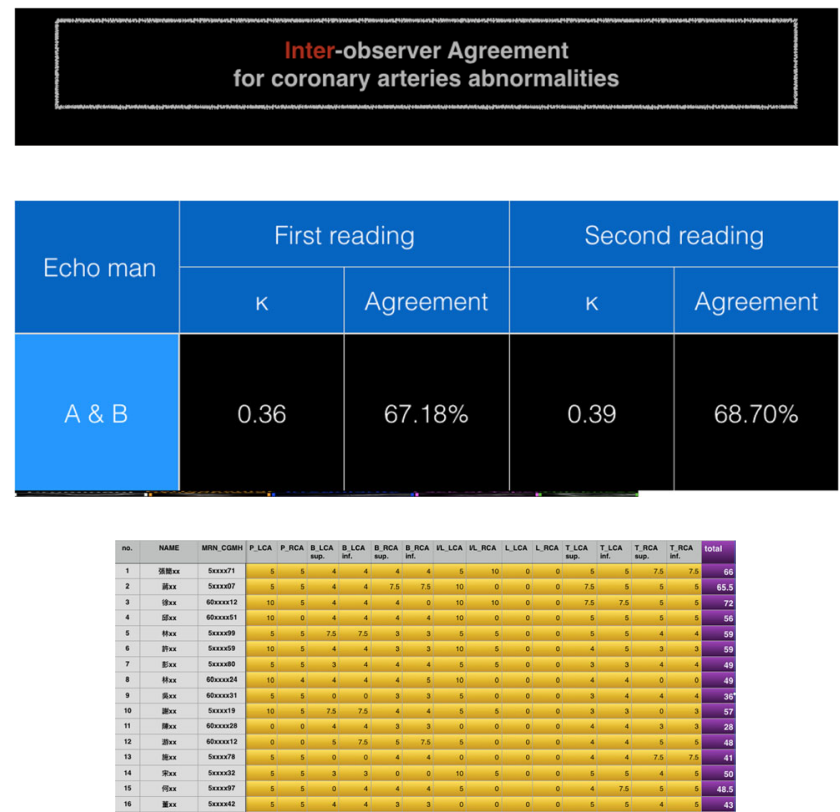


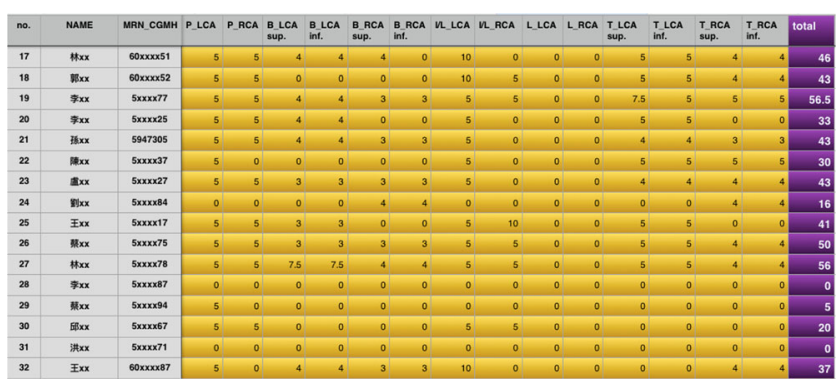

\section{FOCUS scoring system (total : 100 points)}

\begin{tabular}{|c|c|c|c|c|}
\hline $\begin{array}{c}\text { Prominent } \\
20\end{array}$ & $\begin{array}{c}\text { Brightness } \\
40\end{array}$ & $\begin{array}{c}\text { Irregularity } \\
10\end{array}$ & $\begin{array}{c}\text { Lack of taper } \\
10\end{array}$ & $\begin{array}{c}\text { Thickness } \\
20\end{array}$ \\
\hline LCA pro. 5 & $\begin{array}{c}\text { L't superior } \\
\mathbf{5 , 1 0}\end{array}$ & saw-like & short segment & equal Aorta wall \\
\hline RCA pro. 5 & $\begin{array}{c}\text { L't inferior } \\
\mathbf{5 , 1 0}\end{array}$ & 5 & 5 & 10 \\
\hline $\begin{array}{c}\text { LCA Aneurysm } \\
10\end{array}$ & $\begin{array}{c}\text { R't superior } \\
\mathbf{5 , 1 0}\end{array}$ & torturous & diffuse/long & > Aorta wall \\
\hline $\begin{array}{c}\text { RCA Aneurysm } \\
10\end{array}$ & $\begin{array}{c}\text { R't inferior } \\
\mathbf{5 , 1 0}\end{array}$ & 10 & 10 & 20 \\
\hline & & & & \\
\hline
\end{tabular}

742

\section{Rheumatology}

Focused optimal coronary Itra sonography (focus): a novel methodology for coronary image evaluation in acute Kawasaki disease

I Hsin Tai ${ }^{1}$, I Chun Lin $^{1}$, Ho Chang Kuo ${ }^{1}$, Mao Hung Lo ${ }^{1}$, Chien Fu Huang $^{I}$, Ying Jui Lin ${ }^{l}$, Hsuan Chang Kuo ${ }^{1}$, Kai Sheng Hsieh ${ }^{1}$

${ }^{1}$ Department of Pediatrics, Chang Gung Memorial Hospital, Taiwan
Background

Acute Kawasaki Disease $(\mathrm{KD})$ may induce coronary artery abnormalities (CAA) in $25-40 \%$ of the patients based on published document. Golden diagnostic criteria of acute $\mathrm{KD}$ with $\mathrm{CAA}$ requires diameter $\mathrm{Z}$ score 2.5 along with perivascular brightness and lack of tapering. However, the systematic evaluation of these parameter is uncertain.

\section{Objective}

To develop a thorough scoring system useful in systematic evaluation of coronary arteries lesion.

\section{Methods}

Thirty-two children with acute KD plus 100 age-match normal children were assigned to 2 experienced cardiologists who wasn't informed the diagnosis for echocardiography(echo) performed by obtaining dynamic cine-loop recording for frame-by-frame analysis and using quality-optimal highest transducer frequency. CAA are scored as following: 1) Prominence 2) Brightness of arterial wall 3) Irregularity arterial lumen or Lack of tapering 4) Thickness of the arterial wall. Weighted kappa statistics were used to analyze interobserver and intra-observer agreement, and the agreement percentages were calculated

Results

Various CAA were present in acute $\mathrm{KD}$, include various degree of luminal prominence in $24(75 \%)$ patients, aneurysm formation in 3(0.9\%) patients, lack of tapering in $22(68 \%$ ) patients, irregularity in $11(34 \%)$, and intimal thickening brightness in $24(75 \%)$ patients. Overall, coronary artery anomalies were present in $30(93 \%)$ of our patients in acute phase of KD. FOCUS score 10 in $90.6 \%$ (29/32) of patients while only $0.6 \%$ $(2 / 32)$ FOCUS score

\section{Conclusion}

The incidence of coronary artery abnormalities in acute $\mathrm{KD}$ is higher than previously reported. With our novel FOCUS scoring method, more detailed, comprehensive and multi-faced features of CAA other than conventional $\mathrm{Z}$ score can be provided. We thus propose a FOCUS score 10 is indicative for acute $\mathrm{KD}$, 